\title{
Bestandes- und Freilandnährstoffeinträge in Zentralsulawesi (Indonesien)
}

Bulk-Depositionsmessungen mittels Passivsammler im Lore Lindu Nationalpark

\author{
Dissertation \\ zur Erlangung des Doktorgrades \\ der Mathematisch-Naturwissenschaftlichen Fakultäten \\ der Georg-August-Universität zu Göttingen
}

vorgelegt von

Stefan Köhler

aus Worms am Rhein

Göttingen 2012 
Referent: $\quad$ Prof. Dr. Gerhard Gerold

Korreferent: Prof. Dr. Hermann F. Jungkunst

Tag der mündlichen Prüfung: 03.07.2012 


\section{Inhaltsverzeichnis:}

Abbildungsverzeichnis IV

Tabellenverzeichnis $\quad$ VI

Abkürzungsverzeichnis $\quad$ VII

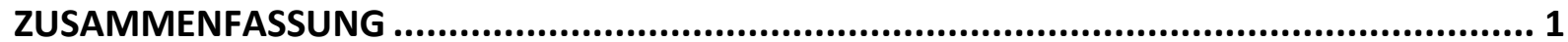

SUMMARY

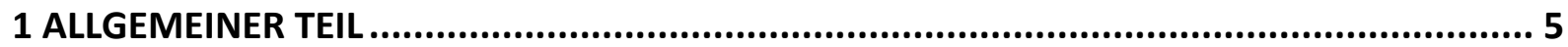

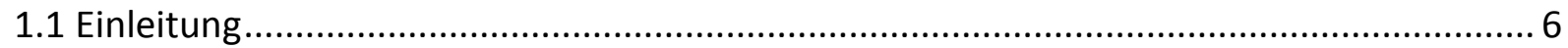

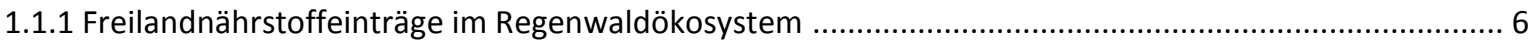

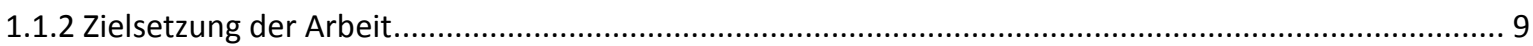

1.2. Methodischer Ansatz und verwendete Software ...................................................... 11

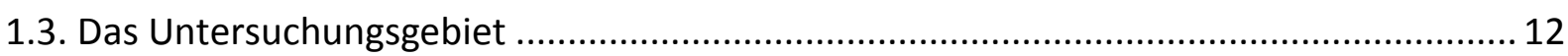

1.3.1 Geoökologische Rahmenbedingungen und Besiedlungsstrukturen ............................................. 13

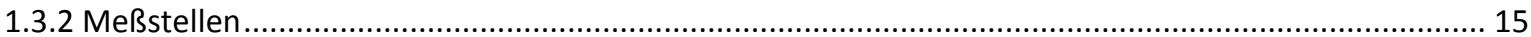

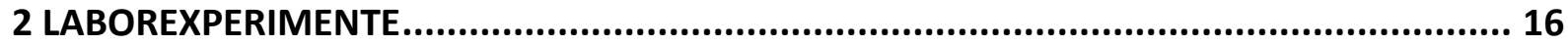

2.1. Die Eignung von lonentauschern zur Fixierung von Regeninhaltsstoffen ...................... 17

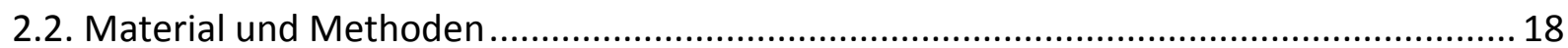

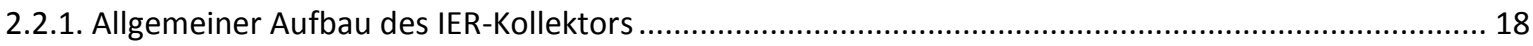

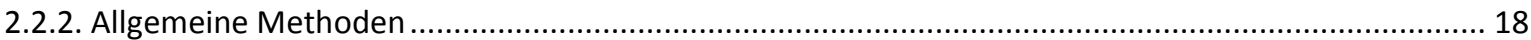

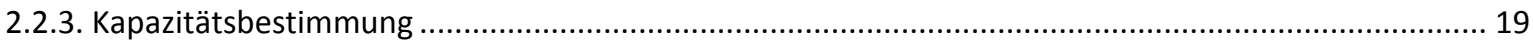

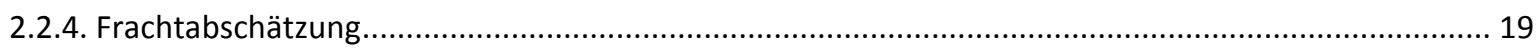

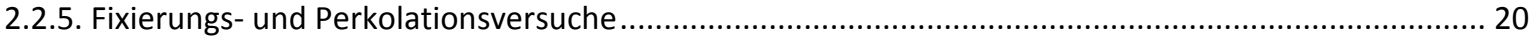

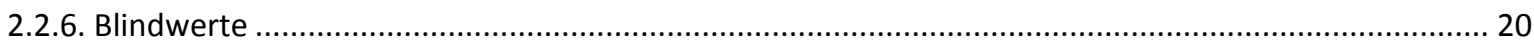

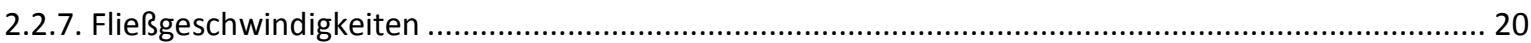

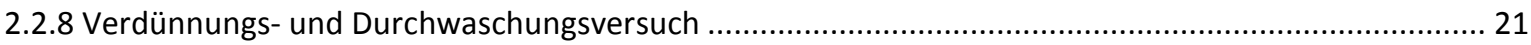

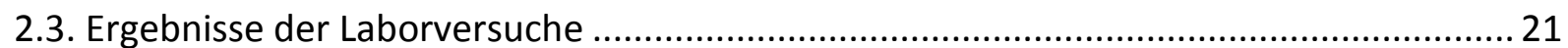

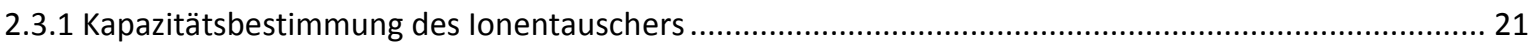

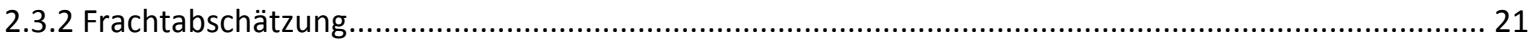

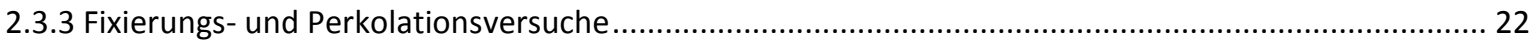

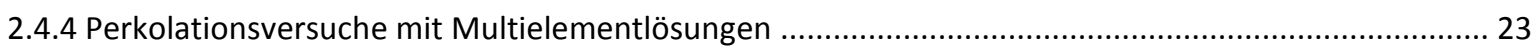




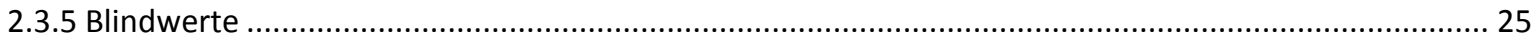

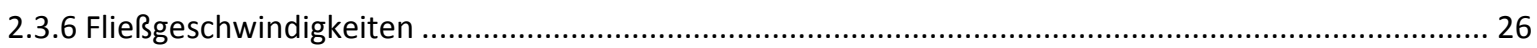

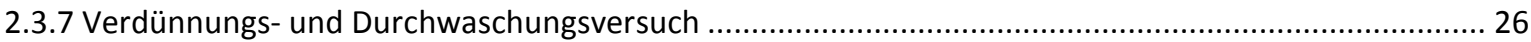

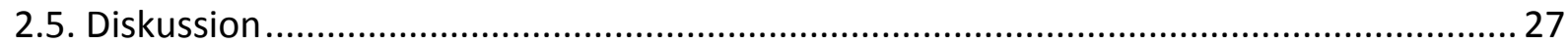

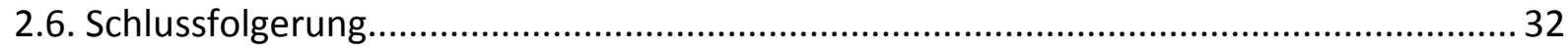

3 ATMOSPHERIC IONIC DEPOSITION IN TROPICAL SITES OF CENTRAL SULAWESI

DETERMINED BY ION-EXCHANGE RESIN COLLECTORS AND BULK WATER COLLECTOR ...... 33

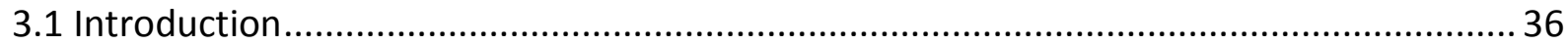

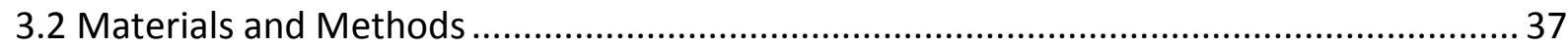

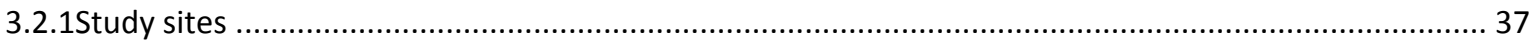

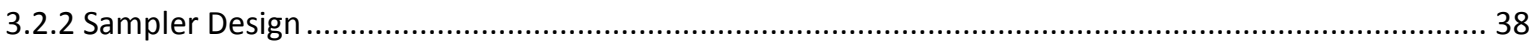

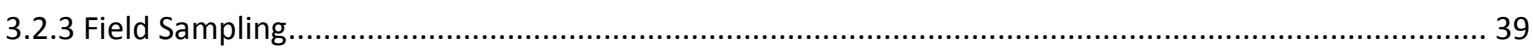

3.2.4 Sample preparation and routine analyses.................................................................................... 39

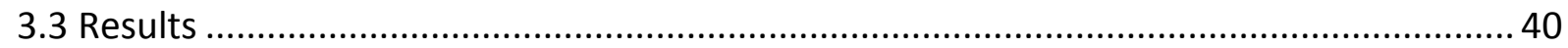

3.3.1 Seasonal variations in precipitation and deposition rates ........................................................... 41

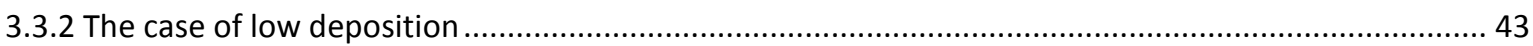

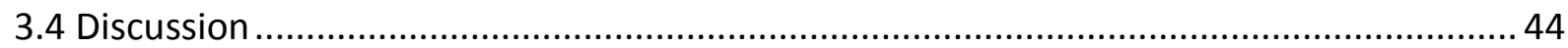

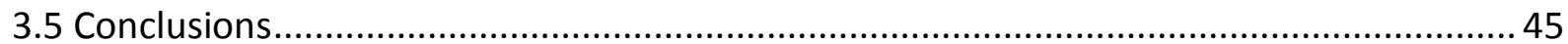

4 REGIONAL ATMOSPHERIC NUTRIENT DEPOSITIONS IN CENTRAL SULAWESI ................. 47

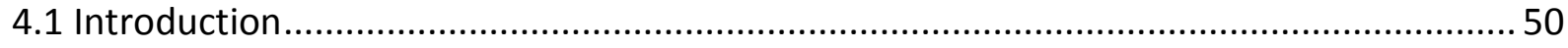

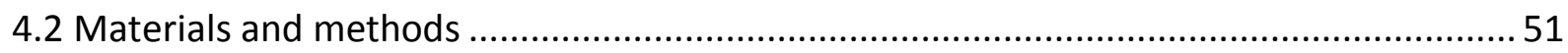

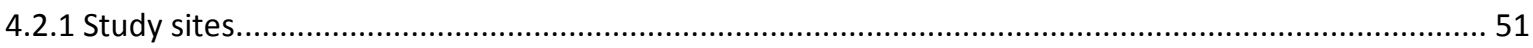

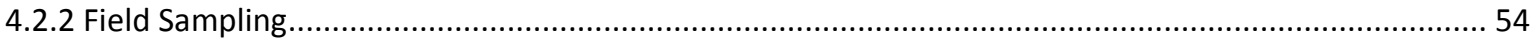

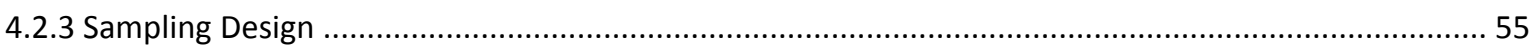

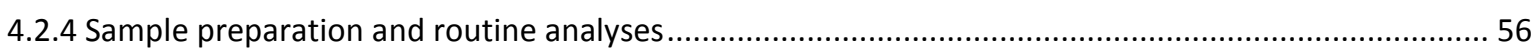

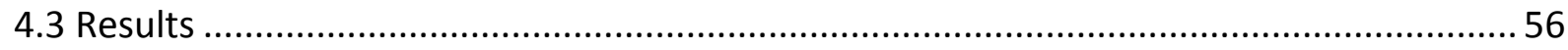

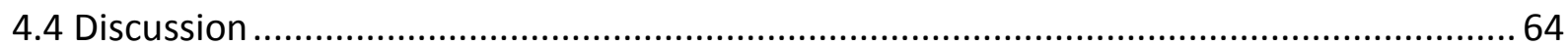

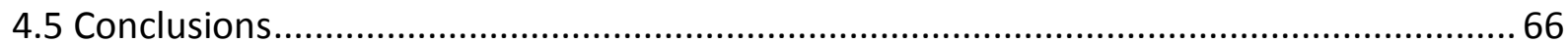

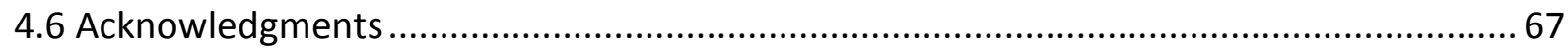


5 BRANDRODUNGSBEEINFLUSSTER NÄHRSTOFFEINTRAG ÜBER BESTANDSNIEDERSCHLAG IN ZENTRALSULAWESI (INDONESIEN) - EIN VERGLEICH VON REGENWALD UND

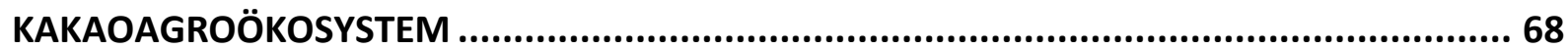

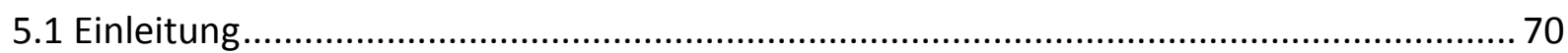

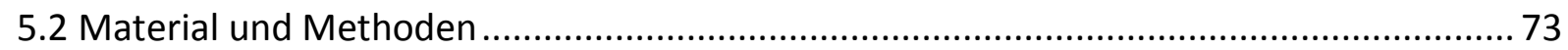

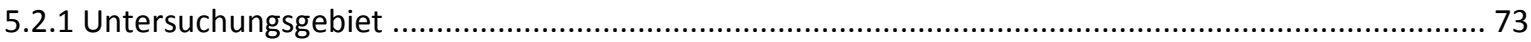

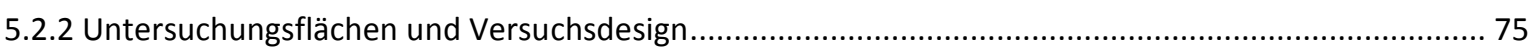

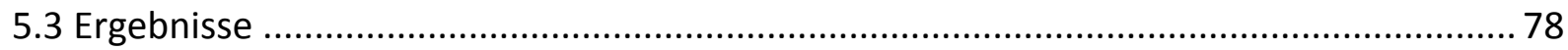

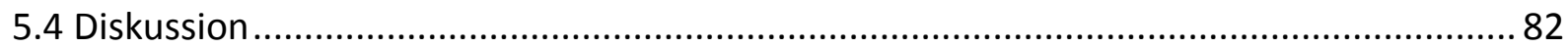

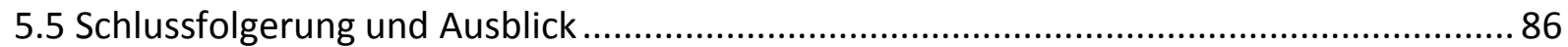

6 ÜBERPRÜFUNG DER FORSCHUNGSHYPOTHESEN ............................................ 88

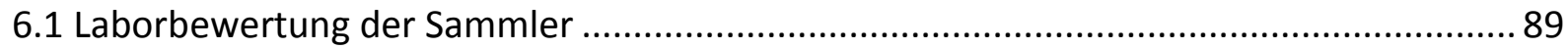

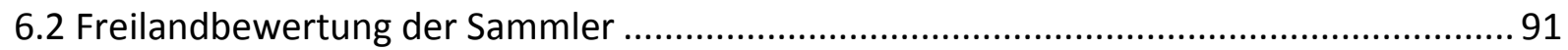

6.3 Heterogenität der Depositionserfassung im Freiland .................................................. 92

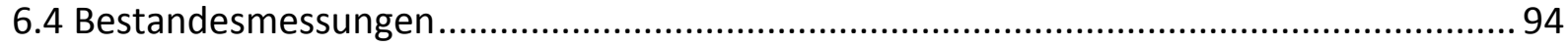

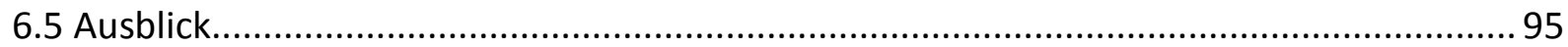

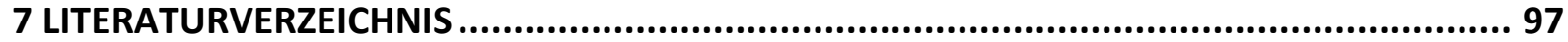




\section{Abbildungsverzeichnis}

Abb. 1.1: Wasser- und Nährstoffumsatz in Regenwaldökosystemen ...................................................

Abb. 1.2: $\quad$ Lage des Untersuchungsgebiets in der Untersuchungsregion des SFB 552 ..............................13

Abb. 1.3: $\quad$ Lage der Standorte im Untersuchungsgebiet und Abholzungsflächen in der Zeit zwischen 1983 und 2007

Abb. 2. 1: IER-Kollektor im Laborversuch und im Freilandeinsatz ................................................... 18

Abb. 2.2: $\quad$ Akkumulationsprofile in den IER-Kollektoren für Natrium .......................................................23

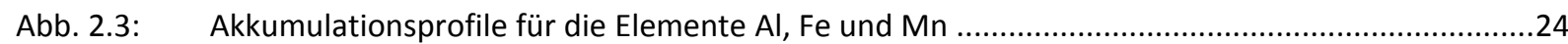

Abb. 2.4: Mittelwerte der Schichtbeladung in den Varianten des Verdünnungsversuches .........................27

Abb. 3.1: $\quad$ Untersuchungsgebiet am Rande des Lore Lindu National Parks in Zentral Sulawesi, Indonesien

Abb. 3.2: $\quad$ Aufbau eines IER-Kollektors

Abb. 3.3.a-e: Monatliche Niederschlagsmenge sowie die Depositionsraten während der Untersuchung für die Elemente $\mathrm{K}, \mathrm{Ca}$ und $\mathrm{NO}_{3}-\mathrm{N}$, erfasst mit IER-Kollektoren oder Niederschlagssammlern

Abb. 3.4: Korrelation zwischen Eintragsdifferenzen der Sammelsysteme IER-Kollektor und BWS-Sammler für Ca und $\mathrm{K}$ am Standort B

Abb.4.1: Monatliche Niederschlagssumme während des Untersuchungszeitraumes am Standort Nopu Open Area und die monatlichen mittleren Niederschläge über ein 10-jähriges Mittel der Standorte Lindu und Kaipirore (1931-1941).

Abb. 4.2: Übersichtskarte des Lore Lindu National Parks mit Brandereignissen im Untersuchungszeitraum (2007 und 2008) in Zenral Sulawesi.

Abb. 4.3: Karte des Lore Lindu Nationalparks mit den Untersuchungsstandorten und den Entwaldungsflächen in unterschiedlichen Zeiträumen .55

Abb. 4.4: $\quad$ IER-Sammler im Feldeinsatz .56

Abb. 4.5: $\quad$ Cluster für das Jahr 2008 (Wards method) auf der Basis aller Elemente (mit null-eins normalisierten Werten) für alle Standorte .59

Abb. 4.6: Cluster (wards method) auf der Basis der normalisierten $\mathrm{NO}_{3}-\mathrm{N}$-Daten aus 2008 ..... .60

Abb. 4.7: $\quad \mathrm{NO}_{3}-\mathrm{N}$-Deposition an den unterschiedlichen Standorten für die Jahre 2007 und 2008, sortiert nach zunehmenden Abstand in Kilometern (km) von Brandrodungsort nahe dem Standort Nopu Plot A.

Abb. 4.8: PCA-Analyse der Elementdepositionen aller Standorte sowie einer farblichen Kennzeichnung der Landnutzungssysteme (rot: Regenwaldstandort in Bariri; blau: Brandrodungsflächen in Nopu; schwarz: landwirtschaftliche Nutzflächen).....

Abb. 4.9: Monatlicher Depositionseintrag verschiedener Elemente in den unterschiedlichen Landnutzungssystemen des Lore Lindu National Parks. 
Abb. 5.1: Wasser- und Nährstoffumsatz in Regenwaldökosystemen (verändert nach Proctor 1987; Nicklas 2006)

Abb. 5.2: $\quad$ Übersichtskarte des Lore Lindu National Parks mit den Standorten für Freiland- und Bestandesdepositionsmessung sowie Brandereignissen im Untersuchungszeitraum (2007 und 2008) in Zenral Sulawesi.

Abb. 5.3: $\quad$ Brandrodungsfläche unterhalb des Versuchsstandortes Nopu Plot C ......................................75

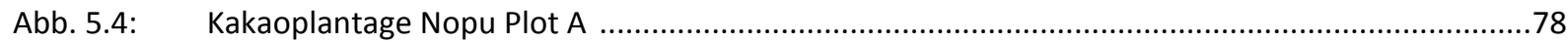

Abb. 5.5 Waldstandort Nopu Plot C mit Versuchsanlage des Partnerporjektes B2...................................75

Abb. 5.6: $\quad$ Passivsammler für Bestandsniederschlag am Standort Bariri Regenwald..................................77

Abb. 5.7: Deposition an den verschieden Messstandorten im Bestandes- und Freilandniederschlag .80

Abb. 5.8: Monatliche Niederschläge und Depositionseinträge während des

Untersuchungszeitraumes an den Standorten Nopu und Bariri für $\mathrm{NO}_{3}-\mathrm{N}$ und $\mathrm{P}$ .82

Abb. 5.9: $\quad$ Saprophytische Besiedlung im Kronenraum am Standort Bariri Wald .83 


\section{Tabellenverzeichnis:}

Tab. 1.1: Nährstoffeinträge als Freilanddeposition in tropischen Regenwaldstandorten (kg $\left.h a^{-1} a^{-1}\right)$

Tab. 1.2: Räumliche Verteilung der Jahresniederschläge $(\mathrm{mm})$ von fünf Messstationen in der Untersuchungsregion in und um den Lore Lindu-Nationalpark nach aus Niklas (2006) nach Berlage (1949)

Tab. 2.1: $\quad$ Fixierungsleistung und Belegungsanteil einer Freilandfrachtabschätzung ..................................21

Tab. 2.2: Gesamtmengen und deren Bilanz aus den Extraktions- und Perkolationsuntersuchungen ...........22

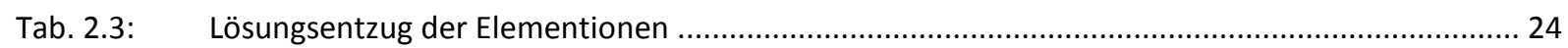

Tab. 2.4: Gehaltswiederfindung in Prozent in den Extrakten der IER-Kollektoren .................................. 25

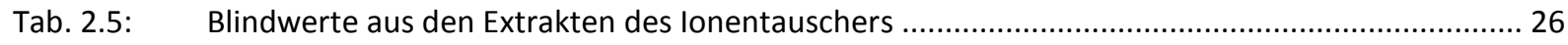

Tab. 2.6: Prozentuale Verarmung Varianten an IER-Kollektoren zum Nährstoffentzug bei hohen

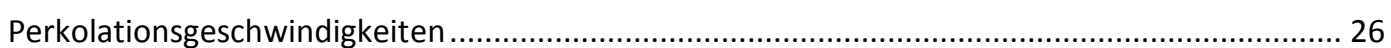

Tab. 2.7: Berechneter Lösungsentzug der Lösungen im Verdünnungsversuch in \% ................................ 27

Tab. 3.1: Blindwerte des Ionentauschermaterials und Extraktionskorrekturfaktoren für Elemente...

Tab. 3.2: Kumulative Depositionsraten der IER-Kollektoren und Niederschlagssammlern in Bulili Standort B, Sulawesi, Indonesien .40

Tab. 3 3: Jahresdeposition in kg ha-1 a-1 für verschiedene Elemente an den drei Versuchsstandorten ..... 41

Tab. 3.4: Prozentanteile der Proben aus Niederschlagssammlern und IER-Kollektoren die unter dem Grenzwert von 0,1 $\mathrm{mg} \mathrm{l}^{-1}$ erfast wurden.

Tab. 4.1: Jährliche Deposition in $\mathrm{kg} \mathrm{ha}^{-1} \mathrm{a}^{-1}$ an den Untersuchungsstandorten im Lore Lindu National Park für die Jahre 2007 und 2008; in 2007 wurden die $\mathrm{NO}_{3}-\mathrm{N}$ nur für 10 Monate erfasst.

Tab. 4.2: Mittlere Elementdeposition für die unterschiedlichen Landnutzungssysteme in $\mathrm{kg} \mathrm{ha}^{-1} \mathrm{a}^{-1}$..... 61

Tab. 4.3: Stoffeinträge $\left(\mathrm{kg} \mathrm{ha}^{-1} \mathrm{a}^{-1}\right)$ und jährliche Niederschlagsmengen tropischer Tieflandregenwälder .. 64

Tab. 5. 1: Nährstoffeinträge als Freilanddeposition in tropischen Regenwaldstandorten $\left(\mathrm{kg} \mathrm{ha}^{-1} \mathrm{a}^{-1}\right) \ldots \ldots \ldots . . . .72$

Tab. 5.2: Position und Eigenschaften der Untersuchungsstandorte .................................................... 76

Tab. 5.3: Jährliche Bestandesdeposition und Freilanddeposition der unterschiedlichen Standorte im kg ha ${ }^{-1}$

Tab. 5.4: Bestandesdeposition und Freilanddeposition in Bariri und Nopu (9 Monate in kg ha $\left.{ }^{1} a^{-1}\right)$

Tab. 5.5: Massenverhältnisse zwischen Bestandesniederschlag und Freilandniederschlag an den Standorten Bariri und Nopu

Tab.5.6: Anreicherungsfaktoren für Elementdepositionen an Regenwaldstandorten und Kakaoplantagen 


\section{Abkürzungsverzeichnis}

\section{Institutionen:}

DFG

STORMA

SFB

Messgeräte:

\section{BWS}

IER-collector
Deutsche Forschungsgemeinschaft

Stability of Rainforest Margins in Indonesia

Sonderforschungsbereich

\section{Einheiten:}

${ }^{\circ} \mathrm{C}$

$m$ ü. NN

$\mathrm{m}$ asl

$\mathrm{mm}$

$\mathrm{mg} \mathrm{m}^{-2}$

$m g m^{-2} a^{-1}$

$\mathrm{g}$

$\mathrm{kg}$

$\mathrm{kg} \mathrm{ha}^{-1}$

kg ha $a^{-1} a^{-1}$

$\mathrm{t}$

$\mathrm{km}$

$\mathrm{mmol}_{\mathrm{c}} \mathrm{g}^{-1}$

$\mathrm{M}$

$\mathrm{ml}$

I

$\min$

$\mathrm{h}$

a

$\mathrm{n}$ bulk water sample

Ion Exchange Resin-collector
Nassdepositionssammler

Passivsammler auf lonentauscherbasis

\section{Grad Celsius}

Meter über Normal Null

Meter über Normal Null

Liter pro $\mathrm{m}^{-2}$ oder Millimeter

Milligramm pro $\mathrm{m}^{-2}$

Milligramm pro $\mathrm{m}^{-2}$ und Jahr

Gramm

Kilogramm

Kilogramm pro Hektar

Kilogramm pro Hektar und Jahr

Tonnen

Kilometer

Millimol Ladung pro Gram

Mol

Milliliter

Liter

Minute

Stunde

Jahr

Anzahl von Versuchswiederholungen

Chemische Symbole:

Al

$\mathrm{Ca}$

$\mathrm{Fe}$

$\mathrm{H}$

$\mathrm{K}$

$\mathrm{Mg}$

$\mathrm{Mn}$

$\mathrm{Na}$

$\mathrm{NaCl}$

$\mathrm{N}$

$\mathrm{NO}_{3}-\mathrm{N}$

$\mathrm{P}$
Aluminium

Kalzium

Eisen

Protonen

Kalium

Magnesium

Mangan

Natrium

Natriumchlorid (Kochsalz)

Stickstoff

Nitrat-Stickstoff

Phosphor 


\section{Zusammenfassung}

Überregionale Nährstoffdepositionen in tropischen Regenwäldern stellen eine wichtige Nährstoffquelle für diese Ökosysteme dar. Mit zunehmenden anthropogenen Stofffrachten in tropische Regenwäldern kommt es zu einer Verschiebung der Nährstoffgleichgewichte, was verbunden ist mit der Veränderung in den Nährstoffkreisläufen und Artenverschiebung.

Die Erfassung von Depositionsfrachten in tropischen Waldgebieten gestaltet sich aufgrund fehlender Infrastruktur in schwer zugänglichen Gebieten schwierig und ist im regionalen Maßstab häufig nicht durchführbar. Problematisch ist sowohl der Probentransport als auch die Bestimmung von gering konzentrierten Nährstoffen in wässrigen Lösungen. Auf lonentauscherbasis wurde ein Passivsammelsystem entwickelt, das Bulkdepositionsproben integrativ über einen Zeitraum von einem Monat im Gelände erfassen kann.

In Labortests konnte gezeigt werden, dass die Sammler elementabhängig zwischen 80\% - 99\% einer anfallenden lonenfracht in mehreren Konzentrationsbereichen am lonentauscher fixieren können. Aufgrund von elementspezifischen Extraktionswiederfindungen zwischen $49 \%$ und $100 \%$ wurden Korrekturfaktoren zur Berechnung der eingetragenen Gesamtnährstofffrachten bestimmt. Anhand unterschiedlicher Bauweisen konnte gezeigt werden, dass eine Kopplung zwischen Perkolationsgeschwindigkeit und Fixierleistung besteht. Versuche mit simulierten Starkregenereignissen von über $300 \mathrm{~mm}$ pro Stunde sowie die Erfassung kleinster Konzentrationen in Lösungen zeigten, dass Ion-Exchange Resin Sammler (IER-Sammler) für den Feldeinsatz geeignet sind. Der Vergleich von Nassdepositionssammlern mit IER-Sammlern im Feldeinsatz zeigte eine elementabhängige Übereinstimmung der Depositionsfrachten zwischen $80 \%$ und $132 \%$ für die Elemente Kalzium (Ca), Eisen ( $\mathrm{Fe}$ ), Magnesium (Mg), Mangan (Mn), Natrium ( $\mathrm{Na}$ ) und Phosphor (P). Für Aluminium (Al) und Nitratstickstoff $\left(\mathrm{NO}_{3}-\mathrm{N}\right)$ wurde in den IER-Sammlern eine mehr als $400 \%$ höhere Depositionsfracht erfasst, was mit den geringen Konzentrationen im Regenwasser sowie einer Artefaktbildung der Stickstoffverbindungen in den Nassdepositionssammlern in Verbindung gebracht wurde. Geringere Depositionen wurde für das Element Kalium (K) (65\%) erfasst. Deutlich belegt werden konnten die höhere Extraktkonzentration und damit die genauere Erfassung der Elemente in den IER-Sammlern. Damit konnte eine Eignung der IER-Sammler für den Feldeinsatz nachgewiesen werden.

Im Rahmen des Sonderforschungsbereiches 552 wurden an 13 Messstellen am Rande und im $2310 \mathrm{~km}^{2}$ großen Lore Lindu National Park in Zentral Sulawesi Depositionsmessungen über einen Zeitraum von 24 Monaten durchgeführt. Zusätzlich wurde über einen Zeitraum von 9 Monaten in 2 Regenwaldbeständen sowie einer Kakaoplantage eine Bestandesmessung durchgeführt. Ziel der Freiland- und Bestandesmessung war es, anthropogene Einträge aus Brandrodungsaktivitäten zu erfassen. 
Mit einem Eintrag von 0,2 bis 0,8 $\mathrm{kg} \mathrm{NO}_{3}-\mathrm{N} \mathrm{ha}^{-1} \mathrm{a}^{-1}$ wurde ein vergleichsweise geringer anthropogener Stickstoffeintrag registriert. Ausnahmen bildeten die Flächen nahe einer aktiven Brandrodungszone mit $\mathrm{NO}_{3}-\mathrm{N}$-Frachten von bis zu $2,7 \mathrm{~kg} \mathrm{ha}^{-1} \mathrm{a}^{-1}$. Haupteintragselement war $\mathrm{Na}$ mit einem Anteil von 50\%-70\% im Untersuchungsjahr 2008. Der hohe Anteil an Na belegt den maritimen Einfluss an den Depositionsfrachten. Im Vergleich zu anderen Regenwaldstandorten liegen die Depositionen in Sulawesi mit Gesamtdepositionen für 2008 von $28 \mathrm{~kg} \mathrm{ha}^{-1} \mathrm{a}^{-1}$ bis $53 \mathrm{~kg} \mathrm{ha}^{-1}$ a'1 meist im unteren Bereich.

Durch die Auswertung der Daten mittels statistischer Methoden konnte ein Unterschied in den Depositionsfrachten verschiedener Landnutzungssysteme gezeigt werden und in drei unterschiedliche Nutzungstypen eingeteilt werden. Als Nutzungssysteme wurden die Typen Brandrodungsgebiete (slash-and-burn), landwirtschaftliche Nutzflächen und tropischer Regenwald ausgewiesen und die Standorte entsprechend zugeordnet. Brandrodungsgebiete werden hauptsächlich durch $\mathrm{NO}_{3}-\mathrm{N}$ und P-Depositionen, in geringerem Umfang auch durch Ca- und K-Depositionen beeinflusst. Der erhöhte Depositionseintrag nimmt mit einem Abstand zur Brandrodungsfläche von $5 \mathrm{~km}$ bereits deutlich ab.

Noch deutlicher konnte der Einfluss nahe gelegener Brandrodungsflächen im Bestandsniederschlag nachgewiesen werden. In Bariri, einem ferngelegenen unberührten Regenwaldstandort, wurden Anreicherungsraten für Ca $(1,3), \mathrm{K}(8,2)$ und $\mathrm{Mg}(3,2)$ gefunden, die nahe denen in der Literatur angegebenen Werten liegen. Die Messungen im Kakaobestand ergaben elementabhängig zwischen 2- bis 10-fach höhere Depositionseinträge, für P eine 20-fach höhere Deposition als am naturnahen Waldstandort, die sich im Bestand des Regenwaldstandortes Nopu nochmals deutlich erhöhten. Ein systematischer Anstieg der Depositionsfrachten und Anreicherungsfaktoren der Elemente mit zunehmender Nähe zum Brandrodungsgebiet wurde nachgewiesen. Die Untersuchung konnte zeigen, dass IER-Sammler geeignet sind, geringe Stoffdepositionen in einer mesoskaligen Fläche zu erfassen und Einflüsse aus Landnutzungssystemen und Brandrodung nachzuweisen. Große Teile des tropischen Regenwaldes in Sulawesi sind noch sehr naturnah und werden kaum von anthropogenen Depositionsfrachten belastet. Durch die Intensivierung der Landwirtschaft, verbunden mit Brandrodung, werden in die Randzonen des Tropenwaldes Nährstofffrachten eingetragen und es kommt zu einer Veränderung der Regenwaldökosysteme. 


\section{Summary}

Supra-regional deposition of nutrients in tropical rainforests represents an important source of nutrients for these ecosystems. Increasing loads of anthropogenic emitted material into the sensitive nutrient cycles of tropical rainforests lead to a shift in the nutrient balance connected with a loss of richness off species.

Measuring of nutrient deposition in tropical areas is mostly difficult because of a lack of infrastructure in remote areas, and in mesoscalic areas it is even impossible. Both the transport of samples to the laboratory and the determination of low-concentrated nutrients in rainwater samples are difficult. Based on ion exchange resin, a passive Ion Exchange Resin-collector (IER-collector) has been developed. The sampler allows collecting bulk deposition samples integrated over a period of approximately one month at field sites.

Laboratory tests showed that the IER-collectors are able to absorb $80-99 \%$ of solute ion loads, depending on element specific properties and different concentrations. Correction factors for the calculation of the all-in-all nutrient deposit load were necessary because in the extraction only $49-100 \%$ of the loaded mass were found depending on element specific characteristics. The comparison different sampler types revealed a correlation between percolation speed and fixation rate on the IER-surface. Tests with simulated hard-rain-events of more than $300 \mathrm{~mm} \mathrm{~h}^{-1}$ and the detection of extremely low nutrient concentrations showed that IER-collectors are suitable for use in field sites.

Nearly identical deposited loads between the two sampling systems (conventional bulk water collectors (BWS-collector) versus IER-collectors) were found for the elements calcium (Ca), iron (Fe), magnesium $(\mathrm{Mg})$, manganese $(\mathrm{Mn})$, sodium $(\mathrm{Na})$ and phosphorus $(\mathrm{P})$. Results of the two measuring systems differed between $80 \%-132 \%$, depending on the element. For aluminium (Al) and nitrate $\left(\mathrm{NO}_{3}-\mathrm{N}\right)$ higher results with more than $400 \%$ were found in the IER-collectors. This could be explained with low concentrations in rainwater samples. The deposition of potassium $(K)$ was underestimated in IER-collectors (65\%). Generally higher deposition loads were found in IER-collectors compared to BWS-collectors. IER-collectors deliver more precise result because a higher concentration of elements in extraction solutions were better detectable at analyse instruments.

In the framework of the Sonderforschungsbereich (SFB) 552, at 13 sites of the $2310 \mathrm{~km}^{2}$ Lore Lindu National Park in Central Sulawesi, nutrient deposition was measured during 24 months. Additionally in two rainforest sites and one cocoa plantation throughfall measurements were performed over a period of 9 months. The aim was to detect anthropogenic depositions arising from slash-and-burn activities. 
For nitrate a relatively low deposition of 0.2 to $0.8 \mathrm{~kg} \mathrm{NO}_{3}-\mathrm{N} \mathrm{ha}^{-1} \mathrm{a}^{-1}$ suggests little anthropogenic influence. However, areas close to slash-and-burn activities showed exceptionally high deposition rates of up to $2.7 \mathrm{~kg} \mathrm{ha}^{-1} \mathrm{a}^{-1}$. The element with the highest deposition rate in 2008 was sodium with $50-70 \%$ of the total deposited mass. This is probably related to the maritime influence of the surrounding ocean. The rainforest in Sulawesi shows lower total depositions with a range of $28 \mathrm{~kg} \mathrm{ha}^{-}$ ${ }^{1} \mathrm{a}^{-1}$ bis $53 \mathrm{~kg} \mathrm{ha}^{-1} \mathrm{a}^{-1}$ compared with other rainforests worldwide.

By statistical analysis differences between different land use systems could be verified. The different amount of deposition allows characterizing three distinct land use systems.

Even clearer the influence of close slash-and-burn areas could be shown in throughfall sites. In Bariri, a pristine rainforest in a remote area, enrichment for $\mathrm{Ca}(1,3), \mathrm{K}(8,2)$ und $\mathrm{Mg}(3,2)$ was measured, comparable with enrichment levels reported in the literature. In comparison with the remoted area of Bariri in the cacao plantation a 2- to 10-times higher deposition was found and especially for $\mathrm{P} \mathrm{a}$ 10-times higher deposition was measured. Even higher enrichment for $\mathrm{P}$ was found in the rainforest of Nopu, the site nearest to the slash-and-burn area. This shows a systematical increase of deposition and enrichment factors with decreasing distance to slash-and-burn areas.

The investigation demonstrated the suitability of IER-collectors for low concentrated nutrients in mesoscalic areas as well as for detecting different land-use types. Large parts of tropical rainforests in Sulawesi are still very close to natural conditions with low anthropogenic deposition inputs. By intensification of agriculture, connected with slash-and-burn events, higher nutrient inputs are carried to the borderlines of the rainforests. This leads to a change of the fine-balanced ecosystems of rainforests. 
1 Allgemeiner Teil

1 Allgemeiner Teil 


\subsection{Einleitung}

Weltweit ist eine permanente Verringerung tropischer Regenwälder und eine zunehmende Ausweitung der landwirtschaftlichen Nutzfläche zu beobachten. Viele dieser Regenwaldstandorte sind einzigartige Ökosysteme, die eine große Artenvielfalt aufweisen (Kessler et al., 2005) und zum Teil einzigartige Habitate verkörpern (Waltert et al. 2004; Schulze et al. 2004).

Mit der Entwaldung werden die biogeochemischen Kreisläufe direkt betroffener und benachbarter Ökosysteme verändert, verbunden mit einer Vielzahl ökologischer Folgen für die Ökosystemservicefunktionen im regionalen und globalen Maßstab (Rockström et al. 2009; Corre et al. 2006). Zu den Störungen gehört neben den veränderten Wasserkreisläufen auch ein massiver Eingriff in die Nährstoffkreisläufe (Gutzler 2011; Clark 2004), die zu negativen Konsequenzen auch für die nachfolgenden Landnutzungssysteme führen können. Regenwaldrodung zugunsten annueller Kulturen oder eines Anbaus von Monokulturen für den Export wie für die Energiepflanzenproduktion führt zum Verlust von Biodiversität, wobei ein Biodiversitätsverlust durch den Anbau verschiedener Arten mit besonderen Anbaupraktiken teilweise gemildert werden kann (Steffan-Dewenter et al.2007). Neben dem aktiven Eingriff in bestehende Regenwaldhabitate durch Abholzung oder Brandrodung werden die Nährstoffumsätze in intakten Regenwäldern durch den Eintrag von Nährund Schadstoffen durch Wind (Aerosole) und Regen beeinflusst (Boy et al. 2008a; Da Rocha et al. 2005)

\subsubsection{Freilandnährstoffeinträge im Regenwaldökosystem}

Ökosysteme sind nur teilweise geschlossene Kreislaufsysteme und stellen offene Systeme dar, in die durch Niederschläge und Trockendeposition Nähr- und Schadstoffe eingetragen werden (Boy et al. 2008a). Im System zirkulieren die Nährstoffe in internen Kreisläufen oder werden in pflanzlicher Biomasse gespeichert (Abb. 1.1). Ausgetragen werden Nährstoffe als Staub oder Bodenpartikel von Feststoffen und Biomasse oder durch gelöste Nährstoffe im Sickerwasser. Die externe Nährstoffbilanz wird bei Vernachlässigung lateraler Wasser- und Stoffumsätze (keine bis geringe Hangneigung) durch den Niederschlagsinput (Freiland- oder Bestandsniederschlag) und Streueintrag sowie den Sickerwasseraustrag unterhalb der Durchwurzelungszone geprägt. Nachhaltige Landnutzungssysteme sind daher auf eine ausgeglichene oder positive externe Nährstoffbilanz angewiesen (Gerold 2009; Gerold 2010). Für den atmosphärischen Nährstoffeintrag sind sowohl die makroklimatischen (Saharastäube in Amazonien, Herrmann et al. 2010; Boy et al. 2008b; Rollenbeck 2010), regionalen (Harmattan in Westafrika, Fischer et al. 2008) wie auch lokalen Bedingungen (Verkehr, Landwirtschaft, Brandrodung, Aas et al. 2007; Ayers et al. 2002; Rollenbeck 2010) von Bedeutung. Dass anthropogen verursachte Stoffeinträge selbst in entlegene natürliche Ökosysteme transportiert werden, konnte in mehreren Untersuchungen nachgewiesen werden (Aas et al. 2007; Sundarambal et al. 2010). 


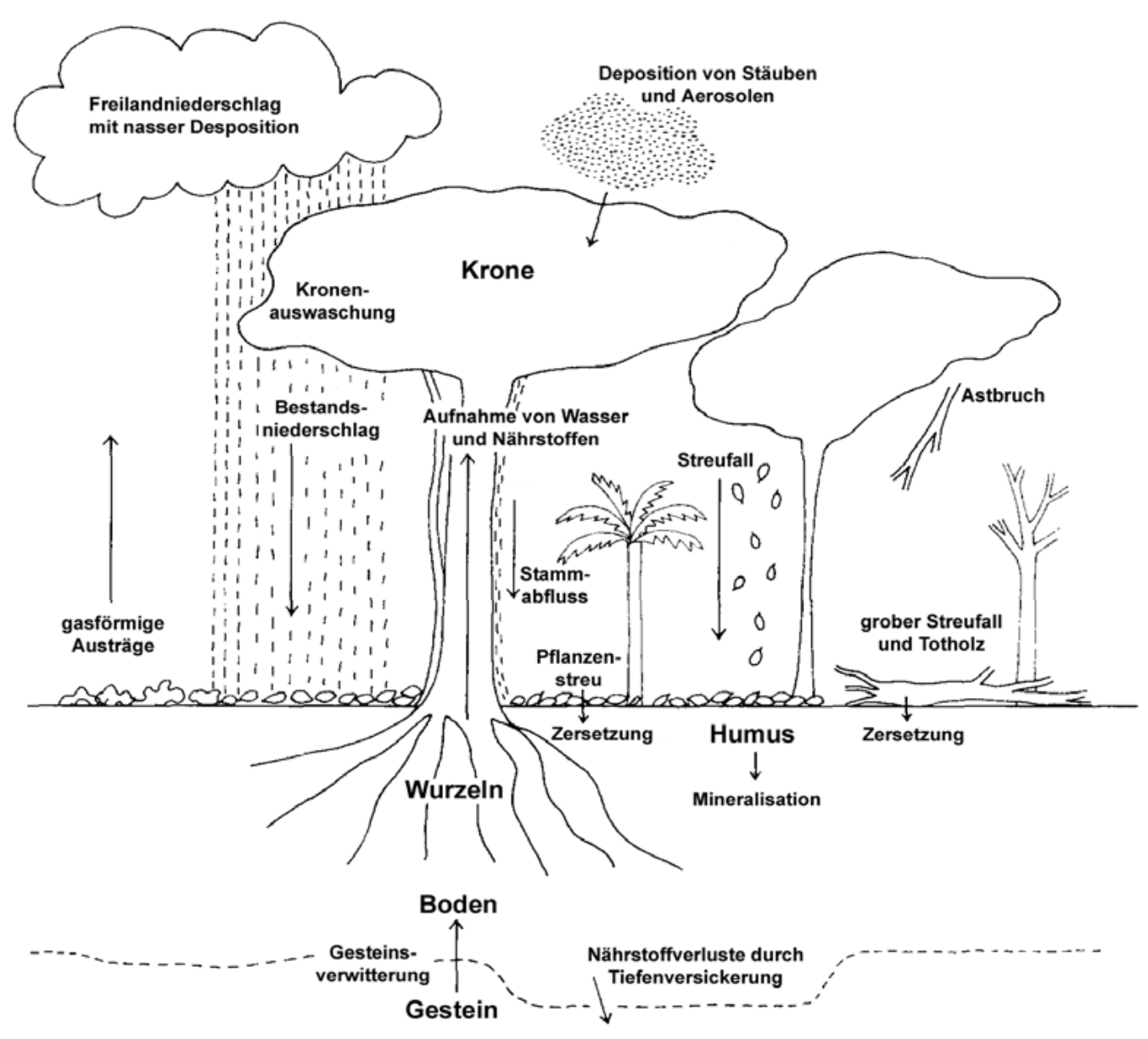

Abb. 1.1: Wasser- und Nährstoffumsatz in Regenwaldökosystemen (verändert nach Proctor 1987; aus Nicklas 2006)

Die quantitative Bedeutung globaler Nährstoffexporte wird anschaulich von Hermann et al (2010) dargestellt. So werden beispielsweise jährlich ca. 200 Mio. t Staub aus der Sahara in Richtung Amazonasbecken exportiert. Faktoren wie Windrichtung und Windstärke, aber auch Relief und Entfernung zur Emissionsquelle bedingen stark unterschiedliche Depositionsmengen in tropischen Regenwäldern weltweit. Bestandseinträge, gemessen in unterschiedlichen Standorten weltweit mit Depositionen zwischen 1 - $28 \mathrm{~kg} \mathrm{ha}^{-1} \mathrm{a}^{-1}$ für Ca (Dezzo et al. 2006; Ashagrie and Zech 2010), 0,3 - 5,5 kg ha $a^{-1} a^{-1}$ für Mg (Schroth et al. 2001; Hofhansel et al. 2011), 1 - $18 \mathrm{~kg} \mathrm{ha}^{-1} \mathrm{a}^{-1}$ für K (Filoso et al. 1999; Hafkenscheid 2002), 2,5 - $75 \mathrm{~kg} \mathrm{ha}^{-1} \mathrm{a}^{-1}$ für Na (Ashagrie and Zech 2010; Calil et al. 2010) 1 - $10 \mathrm{~kg} \mathrm{ha}^{-1}$ $\mathrm{a}^{-1}$ für $\mathrm{NO}_{3}-\mathrm{N}$ (Germer et al. 2007; Oziegbe et al. 2011) und 0,1 - $9 \mathrm{~kg} \mathrm{ha}^{-1} \mathrm{a}^{-1}$ für P (Schroth et al. 2001; Calil et al. 2010) zeigen die Spannbreite der Depositionen und damit den unterschiedlichen Nährstoffstatus tropischer Waldstandorte auf. Einen globalen Überblick der Freilanddepositionen in tropischen Regenwäldern zeigt Tab. 1.1. 
Tab. 1.1: Nährstoffeinträge als Freilanddeposition in tropischen Regenwaldstandorten $\left(\mathrm{kg} \mathrm{ha}^{-1} \mathrm{a}^{-1}\right)$

\begin{tabular}{|c|c|c|c|c|c|c|c|c|}
\hline & $\begin{array}{c}\text { altitude } \\
\text { (m a.s.I.) }\end{array}$ & $\begin{array}{c}\text { rainfall } \\
\text { (mm per year) }\end{array}$ & $\mathrm{Ca}$ & $\mathrm{Mg}$ & $\mathrm{K}$ & $\mathrm{Na}$ & $\mathrm{NO}_{3}-\mathrm{N}$ & $\mathrm{PO}_{4}-\mathrm{P}$ \\
\hline \multicolumn{9}{|l|}{ 1-4: continental } \\
\hline Brazil-Manaus (1) & 50 & 2622 & 0.80 & 0.3 & 2.60 & - & 1.40 & 0.07 \\
\hline Brazil-Rio Negro (2) & 90 & 2900 & 2.50 & 0.4 & 0.70 & 2.50 & 0.80 & - \\
\hline Brazil-Rondonia (3) & 143 & 2300 & 17.50 & 1.60 & 8.70 & 3.40 & 0.80 & - \\
\hline Venezuela Gran Sabana (4) & 1300 & 2548 & 0.80 & 1.30 & 2.20 & - & 1.30 & 0.00 \\
\hline \multicolumn{9}{|l|}{ 5-10: marine } \\
\hline Brazil-Rio Grande do Sul (5) & 175 & 1588 & 11.20 & 3.70 & 18.60 & 42.50 & 3.20 & 8.9 (P2O5) \\
\hline Brazil-Mata Atlantica (6) & 10 & 2235 & 5.60 & 1.60 & 6.40 & 75.20 & - & - \\
\hline Costa Rica SW (7) & 70 & 5810 & 20.00 & 5.40 & 6.20 & 6.90 & 2.00 & 0.4 \\
\hline Costa Rica Monteverde (8) & 1500 & 3191 & 5.80 & 2.40 & 3.00 & 20.50 & 1.70 & 0.05 \\
\hline Panama Cord.Central (9) & 1200 & 3510 & 27.90 & 4.10 & 13.50 & 63.50 & - & 0.7 \\
\hline Puerto Rico (9) & 425 & 3750 & 21.80 & 4.90 & 18.20 & 57.20 & - & - \\
\hline Kamerun Korup NP(10) & 150 & 5370 & 9.30 & 5.30 & 7.80 & - & 1.50 & 1.10 \\
\hline Nigeria Ife-Ife (11) & 215 & 1413 & 5.80 & 2.50 & 5.40 & 15.70 & 10.40 & 1.70 \\
\hline Malaysia (9) & 870 & 2700 & 4.00 & 1.20 & 4.00 & - & 5.00 & 0.10 \\
\hline $\begin{array}{l}\text { Sulawesi Lore Lindu National park } \\
\text { (this investigation) }\end{array}$ & 629- 1375 & $\sim 2500$ & $4.6-19-4$ & $0.9-3.2$ & $1.6-10.6$ & $6.8-21.1$ & $0.1-2.7$ & $0.6-2.0$ \\
\hline
\end{tabular}

(1) Schroth et al. 2001; (2) Filoso et al. 1999; (3) Germer et al. 2007; (4) Dezzo et al., 2006; (5) (Calil et al. 2010(6) Scheer 2011; (7) Hofhansel et al. 2011; (8) Hafkenscheid 2002; (9) Ashagrie et al. 2010 (10) Chunyong et al.2004 (11) Oziegbe et al. 2011, nach G.Gerold, persönliche Mitteilung

Im Allgemeinen sind tropische Regenwaldstandorte meist auf stark verwitterten Böden mit geringen Nährstoffnachlieferungsvermögen anzutreffen (Odum 1999; Aas et al. 2007; Jaramillo et al. 2003; Whitmore 1993; Luizao et al. 2004) und nur 25\% tropischer Regenwälder sind auf fruchtbaren Böden zu finden (Vitousek et al., 1986).

Stark verwitterte Standorte zeichnen sich häufig durch eine geringe Sorption von Nährstoffen, im Besonderen der kationischen Makronährelemente, aus und unterliegen einer starken Auswaschung. Die hohe Mobilität einzelner Nährstoffe und eine geringe Nährstoffnachlieferung der Böden können zu einer Nährstofflimitierung an Standorten tropischer Wälder führen. Der Nährstoffumsatz ist durch einen schnellen internen Nährstoffkreislauf über Wurzelwasseraufnahme, Biomasseaufbau und Mineralisierung des Bestandsabfalls sowie Nährstoffanreicherung im Bestandsniederschlag geprägt (Proctor 1987). Atmosphärische Nährstoffeinträge können daher eine wichtige Funktion in der Nachlieferung essentieller Nährelemente darstellen (z.B. P, Mn), aber auch Ökosystemstörungen mit Artenverschiebung auslösen (z.B. N-Düngung) (Phoenix et al. 2006). Neben den natürlichen Einträgen werden seit längerem auch Depositionseinträge anthropogener Herkunft in natürlichen Waldökosystemen gemessen. Besonders der Eintrag von Phosphor- und Stickstoffdepositionen in nährstofflimitierte Ökosysteme führt zu einer Verschiebung der Nährstoffangebote bis hin zur Eutrophierung eines Systems. Eine längerfristige Überhöhung von Nährstoffeinträgen führt 
wiederum zu einer Artenverschiebung oder gar zu einer Reduzierung der Biodiversität. (Veldkamp et al. 2008). Um die Bedeutung der Nährstoffdepositionen auf ein Ökosystem erfassen zu können, sind neben der Höhe des Eintrages auch die Quellen und die Transportstrecken von Bedeutung. In entlegenen Waldstandorten in China wurden anthropogen verursachte Depositionen nachgewiesen, die zeigen, dass anthropogene Eingriffe durch Nährstoffdepositionen an Standorten ohne direkten menschlichen Kontakt möglich sind (Aas et al. 2007; Fan et al. 2009).

\subsubsection{Zielsetzung der Arbeit}

Die vorliegende Arbeit wurde im Rahmen des Sonderforschungsbereichs (SFB) 552 „Stabilität in Waldrandzonen" in Zentralsulawesi / Indonesien als Teilprojekt der Abteilung Landschaftsökologie der Universität Göttingen durchgeführt.

Eine Bestimmung der Nährstoffdeposition im Nationalpark Lore Lindu war zunächst nur in Einzelprojekten in lokal begrenzten Untersuchungen als Zusatzinformation vorgesehen. Es bestand allerdings die Forderung, eine möglichst flächendeckende Depositionserhebung in und um den Nationalpark Lore Lindu als Ergänzung der Forschungsbeiträge zu Nährstoff- und Kohlenstoffkreisläufen sowie regionaler Klimaanalyse zu erheben. Um die Fläche des Nationalparks von $2310 \mathrm{~km}^{2}$ repräsentativ beproben zu können, wurden die Standorte des bestehenden Klimamessnetzes zur Depositionserfassung mit genutzt (Teilprojekt B7).

In den Tropen ist die Erfassung der Nährstoffgehalte direkt aus den Niederschlagswässern problematisch, da neben der zum Teil schweren Erreichbarkeit der Standorte ein aufwendiger gekühlter Transport von Wasserproben stattfinden muss. Durch Pilz- und Algenwachstum in den Sammelbehältern kann es, vor allem bei Langzeitbeprobungen, zu einer Veränderung der Nährstoffgehalte kommen. Das Sammeln von Wasserproben mittels automatisierter Einrichtungen ist durch mangelnde Stromversorgung sowie Gerätetransport an abgelegene Standorte problematisch. Die Ausdehnung des Messnetzes zeigte schnell, das eine regelmäßige Wasserbeprobung in den Regensammlern der Messstellen aufgrund der Entfernungen in 14 tägigen Intervallen finanziell und personell längerfristig nicht möglich war.

Die geringen Nähr- und Schadstoffkonzentrationen erschwerten darüber hinaus eine exakte Bestimmung. Makroelemente wie Kalium, Natrium, Kalzium oder Magnesium können aufgrund höherer Konzentrationen in Niederschlagswässern noch genau bestimmt werden. Problematisch kann eine Konzentrationsbestimmung von Elementen wie Aluminium oder Eisen sein und ist es sicherlich auch für Mangan aufgrund der geringen gelösten Mengen im Regenwasser (Gutzler 2011; Nicklas 2006). Ähnliches gilt für anthropogen eingetragene Stoffe wie Nitrat-N oder Phosphor, die bei hohen Belastungen im Einzelfall durchaus erfasst werden können, allerdings bei der Messung von Hintergrundwerten unterhalb der Bestimmungsgrenze nicht detektierbar sind. Hohe Niederschlagsereignisse in den Tropen verstärken zudem den Effekt der Verdünnung der Konzentrationen (Fan et al. 2009) und führen darüber hinaus zu einem unsicheren Endergebnis. Die geringen Konzentrationen sind zur Erfassung der Depositionsmenge mit der großvolumigen Regenmenge zu multiplizieren und führen zu einer weiteren Unschärfe des Endergebnisses. Zu beachten ist auch, dass bei der Erfassung der Bulk-Deposition aus Niederschlagsge?wässern nur die 
leicht löslichen Fraktionen der Trockendeposition erfasst werden. Depositionsanteile wie beispielsweise schwer wasserlösliche Oxide (Mangan- oder Eisenoxide) können nicht erfasst werden. Als Folge dieser Vorgaben sollte ein Passivsammler entwickelt werden, der aus lokal beschaffbarem Material besteht, kostengünstig ist, längere Zeit im Feld verbleiben kann und die Nährstoffdepositionen von Nass- und Trockendeposition (Bulkdeposition) quantitativ erfasst. Um Stoffverluste aus den deponierten Einträgen zu vermeiden, sollte der Passivsammler Regenwasser eliminieren und Nährstofffrachten konservieren. Dazu wurde die Idee der Passivsammler auf Ionentauscherbasis von Crabtree und Trugdill (1981) aufgegriffen. Die Integration der Stoffeinträge über einen längeren Zeitraum auf dem Ionentauscher sollte daneben eine Erhöhung der zu erfassenden Stoffkonzentrationen bewirken und analytisch leichter fassbar sein. Darüber hinaus konnte erstmals eine mehrere Quadratkilometer große Fläche in den Tropen bezüglich seiner Depositionseinträge in einer monatlichen Zeitauflösung erfasst werden.

Stoffdepositionen werden aufgrund vielfältiger regionaler (z.B. Reliefabschirmung, Luv-Lee-Effekte, Agglomerationseffekte) und lokaler Faktoren (z.B. Brandrodung, gewerbliche Emissionsquellen) nicht homogen in ein Gebiet eingetragen (Da Rocha et al. 2005; Rollenbeck 2010). Erwartet wurden aufgrund des reliefbeeinflussten Klimas und der wechselnden monsunalen Strömungen sowie des See-Landwindregimes stark unterschiedliche lokale Depositionen in Bezug auf Standort und Mineralstoffe.

Hauptanliegen der Arbeit war es, den entwickelten Passivsammler zu testen und zu bewerten und Nährstoffdepositionen in Quantität und Qualität in der Lore Lindu Region zu untersuchen. Es sollte geprüft werden, inwieweit einzelne Landnutzungssysteme sich in den Depositionseinträgen unterscheiden und in welchem Umfang anthropogene Stoffeinträge in Waldökosysteme stattfinden. Dazu wurden folgende Hypothesen formuliert:

\section{Laborüberprüfung der Sammler:}

H1) Die entwickelten Passivsammler können perkolierenden Lösungen Nährstoffe hinreichend entziehen und am lonentauscher festlegen. Dadurch ist eine Nährstofferfassung im Niederschlagswasser möglich.

H2) An den lonentauschern festgelegte Nährstoffe können durch Extraktion quantitativ zurück getauscht werden. Blindwerte können zur Korrektur der Hintergrundwerte genutzt werden.

H3) Es besteht ein Zusammenhang zwischen dem Aufbau, der Fließgeschwindigkeit im Sammler und der Effektivität in der Fixierung von lonen.

\section{Freilandbewertung der Sammler:}

H4) Die Erfassung der Bulk-Niederschlagsdeposition im Freiland zeigt keine Unterschiede zwischen den IER-Passivsammlern und konventionellen Niederschlagssammlern

H5) Durch das integrierende Sammelverfahren der IER-Sammler, verbunden mit der Gewinnung von Extrakten aus den lonentauschern, können höhere Konzentrationen in den Lösungen erreicht werden. Die Erfassung der Konzentrationen wird erleichtert und geringe Eintragsmengen können gemessen werden. 


\section{Heterogenität der Depositionserfassung im Freiland:}

H6) Natürliche Stoffeinträge aus geogenen und marinen Quellen zeigen geringe zeitliche und räumliche Schwankungen, die auf den Abständen zu den Quellen beruhen. Große Unterschiede weisen auf den Eintrag anthropogener Stoffe hin.

H7) Anthropogene Schadstoffe werden auch in den Nationalpark eingetragen und deponiert.

H8) Emissionen aus Brandrodung sind die größte Quelle für anthropogene Nährstoffdepositionen in Sulawesi. Einzelereignisse verändern die Qualität der Depositionszusammensetzung und bestimmen die Jahresdeposition.

\section{Bestandesmessungen:}

H9) Brandrodung bedingt eine erhebliche Elementanreicherung im Bestandsniederschlag.

H10) Die Quantität der Bestandesdeposition wird durch umgebende Landnutzungssysteme bestimmt.

\subsection{Methodischer Ansatz und verwendete Software}

Die Arbeit ist in vier Arbeitsabschnitte gegliedert, die den Aufbau der Arbeit beschreiben. Zunächst musste eine Entwicklung und Überprüfung der Passivsammler durchgeführt werden. Dazu wurden grundlegende Versuche im Labor unternommen, um die Möglichkeiten und Grenzen der IERKollektoren zu erfassen. Da im Feldeinsatz unkontrollierte Bedingungen auf die IER-Kollektoren wirken und Ergebnisse aus dem Labor nur bedingt auf Freilandbedingungen übertragen werden können, wurden in einem zweiten Schritt IER-Kollektoren und klassische Niederschlagstotalisatoren (Bulkdepositionssammler) im Feld an drei Standorten verglichen. Die Ergebnisse wurden herangezogen, um im Labor gemachte Annahmen wie Berechnungsfaktoren zu bestätigen.

Zeitgleich wurden die meteorologischen Stationen des SFB-Messnetzes mit IER-Kollektoren bestückt. Gemessen wurden lokale Einträge, die miteinander in Bezug gestellt wurden, um lokale Depositionsquantitäten zu bestimmen. Durch den Vergleich der Eintragsmengen zwischen den Stationen sollten mögliche Depositionsquellen erfasst und mögliche Interaktionen zwischen unterschiedlichen Landnutzungssystemen erkannt werden.

Die gemessenen Einträge dienten gleichzeitig als Freiflächenreferenz für die Erfassung von Bestandsniederschlägen in zwei Waldstandorten, die sich durch unterschiedlich umgebende Landnutzungssysteme sowie eine weitere Kakaoplantage unterscheiden. Durch die Erfassung der Bestandsniederschläge wurden Kenntnisse der Stoffverlagerung aus angrenzenden Ökosystemen bzw. Landnutzungssystemen gewonnen und Aussagen zur Beeinflussung naturnaher Waldstandorte konnten getätigt werden.

In der vorliegenden Arbeit wurden zwei unterschiedliche Teilbereiche fokussiert und die Arbeit dementsprechend gegliedert. Im ersten Teil finden sich Angaben zur Überprüfung der Leistungsfähigkeit der IER-Kollektoren im Labor- und Geländeeinsatz. Der Teil des Geländeeinsatzes 
ist in Englischer Sprache abgefasst, da dieser Teil in der Zeitschrift "Water, Air and Soil Pollution“ veröffentlicht wurde.

Der zweite Teil greift die Depositionseinträge der Stationen im Untersuchungsgebiet auf und bringt diese in Zusammenhang mit unterschiedlichen Landnutzungssystemen und anthropogen bedingten Einträgen. Dieser Teil ist in Englisch abgefasst und wurde zur Veröffentlichung in der Zeitschrift Erdkunde eingereicht. An dieses Kapitel schließt die Betrachtung der Bestandsdeposition in den Regenwald- und Kakaostandorten an. Der Artikel zur Bestandsdeposition wird in der Zeitschrift GeoÖko veröffentlicht.

Abgeschlossen wird die Arbeit mit einer Überprüfung der angeführten Hypothesen, eingebettet in einer Zusammenfassung der gewonnenen Erkenntnisse aus der Arbeit.

Für die Erstellung dieser Arbeit wurden Programme aus dem Paket Microsoft Office verwendet. Zur statistischen Berechnung wurden die Programe PAST (Hammer, et al., 2001)und SPSS 14 verwendet. Graphiken wurden mit dem Program Xact erstellt.

\subsection{Das Untersuchungsgebiet}

Indonesien besitzt mit einer Bedeckung von 52\% seiner Gesamtfläche (Gutzler 2011; FAO 2011) mit die größten Waldreserven in Südost Asien. Indonesien gehört jedoch zu den Ländern mit dem höchsten Regenwaldrückgang durch Rodung in den letzten 20 Jahren. Nach FAO (2011; 2010) betrug die Entwaldungsrate zwischen $1,6 \%$ bis 2,0\%, wobei Zentralsulawesi eine deutlich geringere Entwaldungsrate aufzeigt (0,6\%, Erasmi et al. 2007). Die Entwaldung in Zentralsulawesi durch Brandrodung (slash-and-burn) geschieht vor allem zur Anlage kleinbäuerlicher Kakaoplantagen (+26\% 2001-2007, Erasmi et al. 2007). 


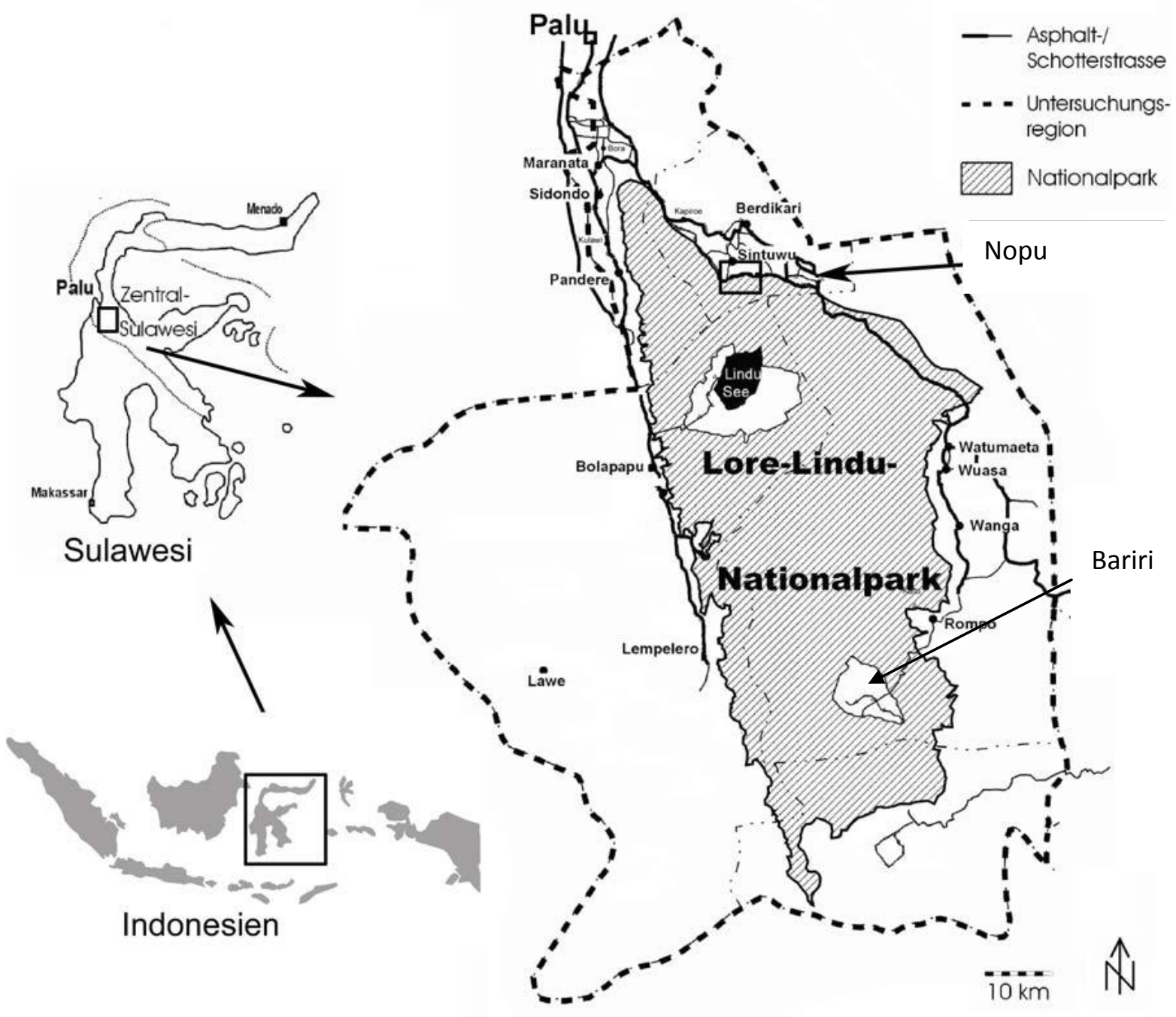

Abb. 1.2: Lage des Untersuchungsgebiets in der Untersuchungsregion des SFB 552 (verändert nach CSIADP basemap 1996 in Weber (2005) aus Nicklas (2006), verändert)

\subsubsection{Geoökologische Rahmenbedingungen und Besiedlungsstrukturen}

Durchgeführt wurden die Untersuchungen auf der Insel Sulawesi, der viertgrößten Insel Indonesiens (Whitten et al. 2002). Der Lore Lindu National Park liegt in unmittelbarer Nähe zum Äquator, $\left(01^{\circ} 15^{\prime}\right.$ bis $01^{\circ} 30^{\prime} \mathrm{S} ; 119^{\circ} 50^{\prime}$ bis $120^{\circ} 20^{\prime} \mathrm{E}$, Abb. 1.2), erstreckt sich über eine Fläche von $2310 \mathrm{~km}^{2}$ und ist mit ca. 90\% Waldbedeckung eines der größten Regenwaldgebiete Sulawesis (Kleinhans 2003; Leemhuis 2005).

Ausgelöst durch die Nordwärtsdrift der Australischen Platte vor 13-19 Millionen Jahren, entstand durch das Zusammentreffen mehrere Inselbruchstücke die Insel Sulawesi. Die letzte Kollision der jungen magmatischen Bruchstücke ereignete sich vor rund 5 Mill Jahren und führte zur Bildung der Palu-Koro-Fault (Whitten et al. 2002; Villenneuve et al. 2002), einem bis heute tektonisch aktiven Grabenbruch, der das Flussbett des Paluflusses bildet und den Nationalpark westlich begrenzt. 
Als Ausgangsgestein zur Bodenbildung wird überwiegend Granit, Andesit und Diorit im Untersuchungsgebiet vorgefunden (Whitten et al. 2002). Im Gegensatz zu vielen anderen Standorten tropischer Regenwälder werden im Untersuchungsgebiet 80\% nährstoffreiche Cambisole gefunden (Mackensen et al. 1999; Dechert et al. 2005; Kleinhans 2003). Auf steileren Hängen, sowie jungen durch seismische Erschütterungen ausgelösten Hangrutschungen werden Leptosole vorgefunden.

Ein ausgeprägtes Gebirgsrelief, mit Höhen zwischen $200 \mathrm{~m}$ ü. NN und $2610 \mathrm{~m}$ ü. NN, welches mit unterschiedlichen montanen und submontanen Wäldern bestanden ist, kennzeichnet den Park. Im Untersuchungsgebiet liegen die Dörfer und Hauptanbauflächen in intramontanen Becken und Tälern. Nach der Klimaklassifikation von Köppen (1923) ist das Gebiet durch ein tropisches Regenwaldklima (Tageszeitenklima) ohne Trockenzeiten gekennzeichnet. Durch den Einfluss der innertropischen Konvergenzzone fallen ganzjährig Niederschläge, wobei die höchsten Niederschläge in den Monaten März bis Juni und November bis Dezember verzeichnet werden (Whitten et al. 2002). Aufgrund der Reliefierung variieren die Jahresniederschläge zwischen über $2000 \mathrm{~mm}$ im Gebirge und $600 \mathrm{~mm}$ in Palu, der Provinzhauptstadt Zentralsulawesis (Berlage 1949; Leemhuis 2005).

Tab. 1.2: Räumliche Verteilung der Jahresniederschläge $(\mathrm{mm})$ von fünf Messstationen in der Untersuchungsregion in und um den Lore Lindu-Nationalpark (aus Niklas 2006 nach Berlage 1949)

\begin{tabular}{l|cccc} 
& Lage & Höhe ü. NN & Jahresniederschlag & Messzeitraum \\
\hline Palu & $00^{\circ} 55^{\prime} \mathrm{S}, 119^{\circ} 54^{\prime} \mathrm{E}$ & 30 & 547 & $1908-1941$ \\
Bora & $01^{\circ} 02^{\prime} \mathrm{S}, 119^{\circ} 57^{\prime} \mathrm{E}$ & 90 & 1042 & $1923-1941$ \\
Kapiroe & $01^{\circ} 19^{\prime} \mathrm{S}, 119^{\circ} 91^{\prime} \mathrm{E}$ & 525 & 1797 & $1919-1941$ \\
Kulawi & $01^{\circ} 266^{\prime} \mathrm{S}, 119^{\circ} 59^{\prime} \mathrm{E}$ & 735 & 2239 & $1916-1941$ \\
Lindu & $01^{\circ} 18^{\prime} \mathrm{S}, 120^{\circ} 05^{\prime} \mathrm{E}$ & 1000 & 2371 & $1931-1941$
\end{tabular}

Die mittlere Tagestemperatur liegt bei $25^{\circ} \mathrm{C}$ bis $26^{\circ} \mathrm{C}$ an der Küste und sinkt mit zunehmender Höhe auf $18^{\circ} \mathrm{C}$ in $1200 \mathrm{~m} \mathrm{NN}$ ab (Nicklas 2006). In der Arbeit von Kleinhans (2003) wurden klimatische Daten im Zeitraum von 2002 bis 2004 für den Standort Nopu im Untersuchungsgebiet erfasst. Kleinhans (2003) gibt eine mittlere Tagestemperatur von $24,2^{\circ} \mathrm{C}$ mit einer Tagesamplitute von $12,2^{\circ} \mathrm{C}$ an. Die mittlere Luftfeuchtigkeit wird mit $87 \%$ angegeben.

Im Gebiet um den Nationalpark sind landwirtschaftliche Flächen vor allem in den Ebenen und Tälern angesiedelt. Whitten et al (2002) führt dies auf die geringe Eignung der steilen Hangflächen zurück. Die Untersuchung von Maertens et al (2006) konnte in der Zeit zwischen 1980 und 2001 einen deutlichen Bevölkerungszuwachs von $66 \%$ im Gebiet nachweisen. In diesem Zeitraum wurden neue landwirtschaftliche Nutzungsflächen über Brandrodung erschlossen, die seit der politischen Dezentralisierung (ab 2000-2002) zunehmend im Randbereich des Lore Lindu Nationalparks stattfand (Erasmi et al. 2007). Im Untersuchungsgebiet lebten nach Leemhuis (2005) ca. 300.000 Personen. Dieser Bevölkerungszuwachs geht auf Umsiedlungsprojekte der indonesischen Regierung in den 80er 
Jahren des vergangen Jahrhunderts zurück, und dauert bis heute durch die Zuwanderung der BugisBevölkerung aus Westsulawesi (Weber 2005) an. In der Zeit von 1972 bis 2002 wurden 15\% der Waldfläche in landwirtschaftliche Nutzflächen umgewandelt (Erasmi, et al. 2004). Die Umwandlung erfolgte von Kleinbauern durch Brandrodungsverfahren (0,6\% $\mathrm{a}^{-1}$; Erasmi et al. 2004).

\subsubsection{Meßstellen}

Im Umkreis des Lore Lindu Nationalparks wurden 12 Messstellen, gekoppelt an das Klimastationsnetz des SFB 552, aufgebaut

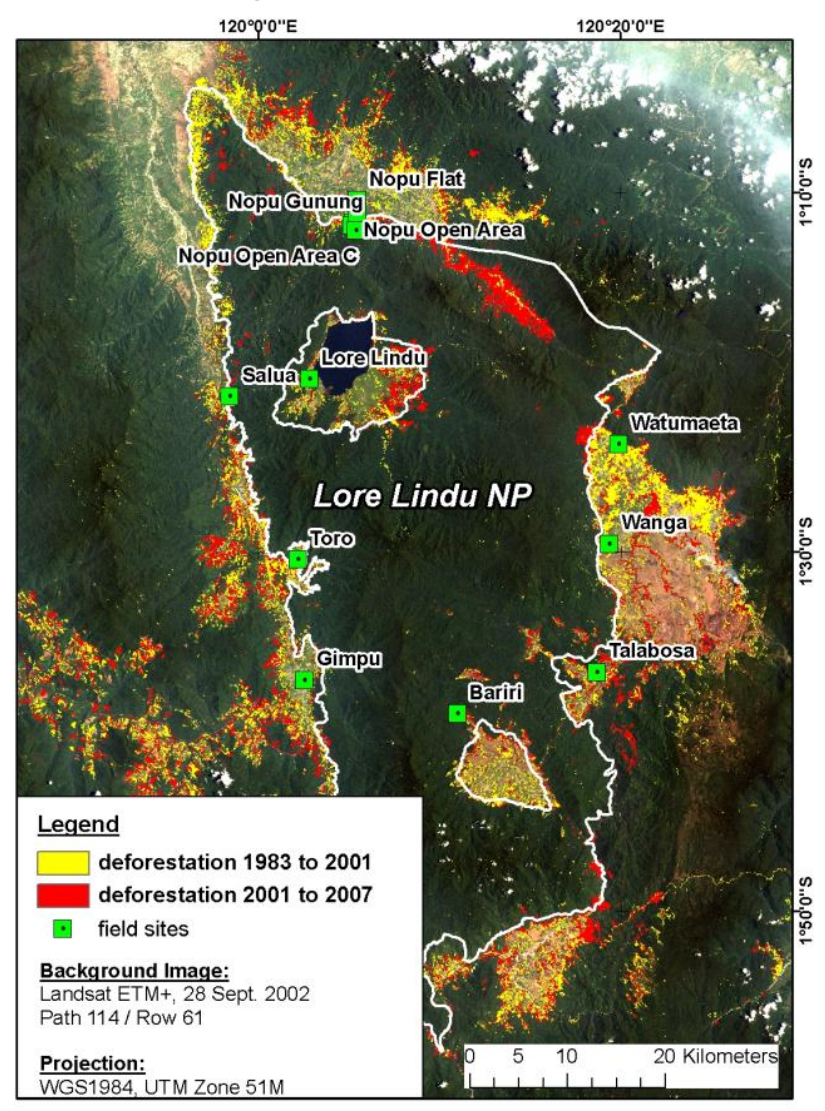

-Abb. 1.3: Lage der Standorte im Untersuchungsgebiet und Abholzungsflächen in der Zeit zwischen 1983 und 2007 (Kartengrundlage Stefan Erasmi, persönliche Mitteilung).

An diesen Standorten erfolgten Nährstoffeintragsmessungen mittels passiver Ionenaustauschersammler (IER-Kollektoren) vom 02.01.2007 bis 02.01.2009 mit einer zunächst zweiwöchentlichen, später monatlichen Probennahme. Aufgrund intensiver Brandrodungsaktivitäten im Bereich der Standorte Nopu wurde das Messnetz ab August 2007 um 2 Messstellen erweitert. Jahresdepositionen können für diese Standorte nur für das Jahr 2008 angegeben werden.

An zwei Waldstandorten (Nopu und Bariri) sowie einer Kakaoplantage (Nopu) wurde eine Bestandsniederschlagsmessung durchgeführt (Bulk-Niederschlagsdeposition). Die Messung erfolgte über 13 Monate beginnend im Mai 2007 bis Juni 2008. Als Referenz wurden nahe gelegene Freilandmessstellen herangezogen. Die Niederschlagsproben wurden 14-tägig gesammelt. 
2 Laborexperimente

2 Laborexperimente 


\subsection{Die Eignung von lonentauschern zur Fixierung von Regeninhaltsstoffen}

Zur Beurteilung des Stoffumsatzes in Ökosystemen und deren Vergleich ist die Kenntnis über atmosphärisch eingetragene Nährstoffe notwendig. Veränderungen von In- und Outputbilanzen im Nährstoffhaushalt sowie Veränderungen der Stoffflüsse in Ökosystemen werden durch die Bestimmung der Stofftransferraten erfasst und beurteilt. Anthropogene Einträge, wie z.B. durch Brandrodung in den Tropen, verändern den Stoffeintrag und damit die Nährstoffflüsse in hohem Maße wie vielfach gezeigt (Larssena et al. 2000; Aas et al. 2007; Krusche et al. 2003).

In den Tropen gestaltet sich die Erfassung der Nährstoffgehalte direkt aus den Niederschlagswässern problematisch (Aas et al. 2007; Gutzler 2011). Neben der zum Teil schweren Erreichbarkeit der Standorte müssen Wasserproben oft aufwendig und ungekühlt transportiert werden. Vielfach angewandte Bulkdepositionssammler können durch personell-logistische Gründe häufig nur zweiwöchentlich oder monatlich beprobt werden, und chemische Umsatzreaktionen in den Proben sind zu erwarten. Durch Pilz- und Algenwachstum kann es, vor allem bei Langzeitbeprobungen, zu einer Veränderung der Nährstoffergebnisse (Nitratumsatz, Phosphor) kommen.

Technische Lösungen sind durch hohe aufzuwendende Sachmittel gekennzeichnet und scheitern oft an mangelnder Stromversorgung, einem schwierigen Gerätetransport vor Ort und stellen durch die regelmäßig durchzuführenden Wartungsarbeiten durch Fachpersonal eine schwer zu realisierende Alternative dar. Datenverluste durch Geräteausfall werden mit zunehmendem Alter der Anlagen häufiger.

Ziel war es, mit den vor Ort (in Zentralsulawesi) verfügbaren Materialien kostengünstige Passivsammler zu bauen, um an entfernt liegenden Standorten Depositionsproben zu sammeln, diese zu konservieren und einen einfachen (vom Gewicht sowie der Logistik) Probentransport zu realisieren, um Aussagen zur Bulkdeposition machen zu können. Es wurde ein Passivsammler auf lonentauscherbasis entworfen, wobei gegenüber den Sammelgeräten von Crabtree und Trudgill (1981) sowie Fenn und Poth (2004) und Smitkin (2004) eine stark vereinfachte Konstruktion bevorzugt wurde.

Um die Eignung der Passivsammler, nachfolgend als Ion Exchange Resin-Kollektors (IER-Kollektor) bezeichnet, zu testen, wurden Laborversuche durchgeführt. Gemessen wurde die Kapazität des Ionentauschers, Blindwerte des Tauschers für verschiedene Elemente und Nährstoffe, die Entzugsleistung des IER-Kollektors aus Multielementlösungen unterschiedlicher Konzentrationen sowie die Gehalte in den Extrakten aus den IER-Kollektoren. Als Extraktionsmittel wurde Schwefelsäure verwendet, welche als Batteriesäure leicht weltweit beschafft werden kann. Zur Bestimmung der notwendigen Ladungskapazitäten wurden Bulkdepositionsdaten von Nicklas (2006) aus dem STORMA-Untersuchungsgebiet, den Waldrandzonen des Lore Lindu Nationalparks in Zentralsulawesi, herangezogen und Nährstofffrachten abgeschätzt. 
Zusätzlich wurde die Eignung der IER-Kollektoren auf intensive Starkregenereignisse untersucht. Dazu wurden Beladungsexperimente mit unterschiedlichen Konstruktionsformen des IER-Kollektors und schnell perkolierender Lösungen durchgeführt.

Um Auswirkungen von sinkenden Stoffkonzentrationen während eines Niederschlagsereignisses auf den IER-Kollektor zu überprüfen, wurde eine mehrfach sich verdünnende Lösung durch den IERKollektor perkoliert sowie bereits beladene IER-Kollektoren mit geringkonzentriertem Wasser durchspült.

\subsection{Material und Methoden}

\subsubsection{Allgemeiner Aufbau des IER-Kollektors}

Aufgebaut ist der IER-Kollektor aus $50 \mathrm{~cm}$ langem, im Innendurchmesser $10 \mathrm{~mm}$ weitem, gewebeverstärktem Kunststoffschlauch, auf den ein Kunststofftrichter mit einem Durchmesser von 190 mm aufgesetzt wurde. Das untere Ende des Schlauches wurde mit einer durch ein Gummiband fixierten Nylongase verschlossen.

Zur Fixierung der Nährstoffe werden die Schläuche mit $50 \mathrm{~g}$ des Mischbetttauschers Amberlit MB 50 befüllt. Zum Schutz vor Insekten und Laubeintrag wird über dem lonentauschermaterial ein Pfropfen aus vorgeglühter Glaswolle eingebracht (Abb. 2.1).

Die Buchstaben 1-5 in der Abbildung bezeichnen die jeweils $2 \mathrm{~cm}$ starken Schichten, in welche das Ionentauscherfüllmaterial zur Analyse im Labor zerlegt wurde.

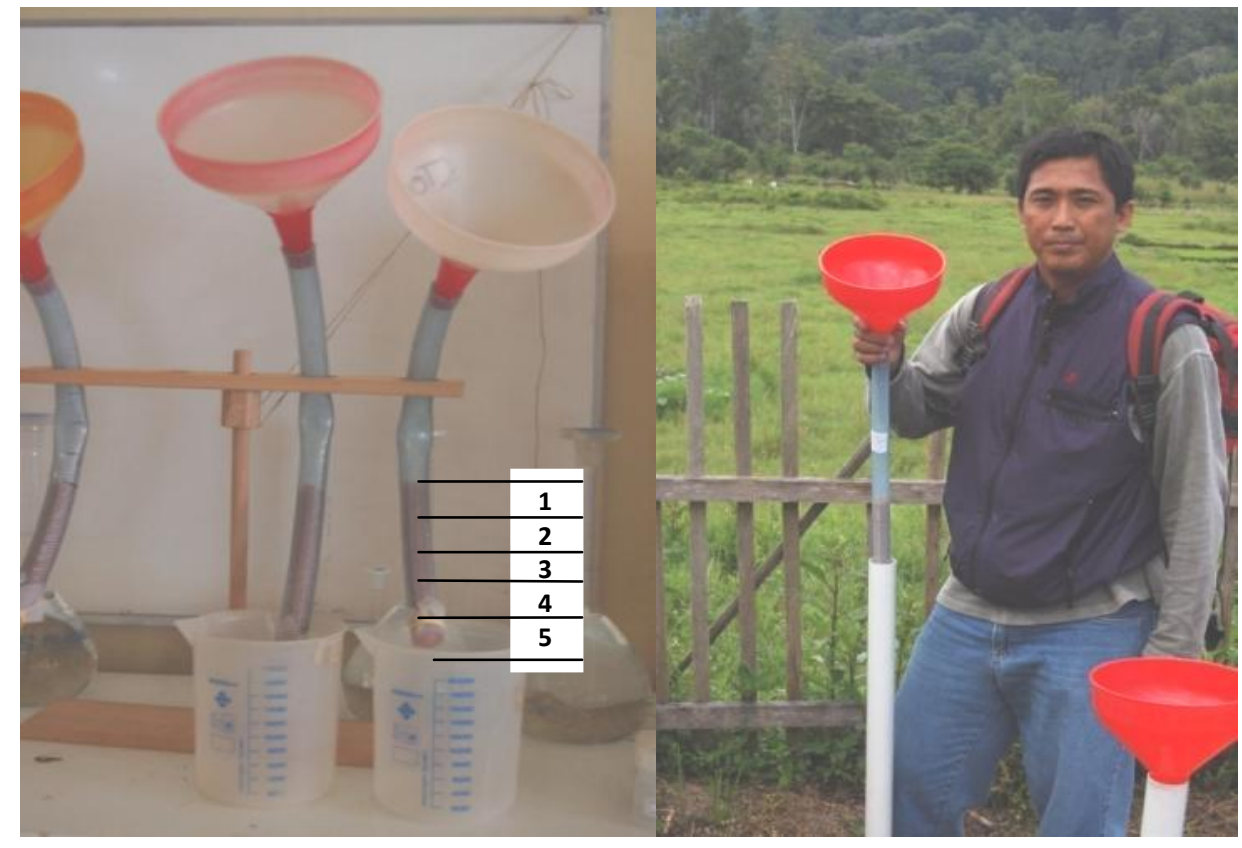

Abb. 2.1: IER-Kollector im Laborversuch und im Freilandeinsatz

Im folgenden ersten Abschnitt werden die Verfahren beschrieben, die für alle durchgeführten Versuche gelten. Wurden davon abweichende Versuchsbedingungen geschaffen, werden diese bei der Beschreibung der Versuche erläutert.

\subsubsection{Allgemeine Methoden}


Zur Bestimmung der Kapazität und den Blindwertbestimmungen wurden Batchexperimente mit anschließender Extraktion der Ionentauscher durchgeführt, welche weiter unten ausführlich beschrieben werden.

Für die Perkolations- und Fixierversuche sowie den Regensimulationsversuchen wurden die IERKollektoren mit unterschiedlich konzentrierten Lösungen beschickt. Aufgrund des großen Wasserbedarfs für die Versuchsdurchführung wurden alle Lösungen mit Wasser aus den Klimaanlagen (AC-Wasser) aufgefüllt. Zu Beginn der Versuche wurde eine Konzentrationsbestimmung der Lösungen durchgeführt.

Nach der Beladung der Ionentauscher wurden in allen Versuchen 5-Ionentauscherschichten mit jeweils ca. $2 \mathrm{~cm}$ Schichtstärke den Röhren entnommen, gewogen und für die Extraktion aliquotiert. In allen Versuchen wurden mit 1.Schicht die Schichten bezeichnet, welche dem Eintrag am nächsten ist. Die Bezeichnung wurde mit 2. bis 5. Schicht nach unten fortgesetzt und 5.Schicht bezeichnet die Schicht direkt über der Nylongaze.

Extrahiert wurden die lonentauscherproben mit einer 1 molaren Schwefelsäure für die Bestimmung von Aluminium (Al), Kalzium (Ca), Eisen (Fe), Kalium (K), Magnesium (Mg), Mangan (Mn), Natrium $(\mathrm{Na})$ und Phosphor (P) am ICP oder einer 1 molaren Kochsalz-Lösung für die Messungen von NitratStickstoff $\left(\mathrm{NO}_{3}-\mathrm{N}\right)$ am Continiuos-Flow-Analyser (Bran\&Lübbe).

Bei allen Versuchen wurden jeweils drei Extraktionsstufen durchgeführt. Lediglich bei den Versuchen mit den sich verdünnenden Lösungen wurden zwei Extraktionsstufen durchgeführt. Dazu wurde pro Extraktionsstufe der lonentauscher mit jeweils $50 \mathrm{ml}$ des entsprechenden Extraktionsmittels versetzt und 30 min extrahiert.

Bestimmt wurden die lonenkonzentrationen an einem ICP-OES 2000 der Firma Perkin Elmer. Nitrat wurde am Continius-Flow-Anayser der Firma Bran \& Lübbe bestimmt. Alle Analysen wurden im Labor des Sonderforschungsbereiches (SFB) 552 in Palu an der Universität Tadulaku durchgeführt.

Durchführung der Versuche im Einzelnen:

\subsubsection{Kapazitätsbestimmung}

Zur Kapazitätsbestimmung wurden drei Proben mit je $5 \mathrm{~g}$ lonentauschermaterial in eine $100 \mathrm{ml} \mathrm{PE-}$ Flasche eingewogen und anschließend mit $100 \mathrm{ml}$ einer 2 Molaren Kochsalzlösung versetzt, 30 min. geschüttelt und die überstehende Lösung dekantiert. Anschließend wurde der lonentauscher dreimal mit jeweils $50 \mathrm{ml}$ Methanol gewaschen und danach mit $50 \mathrm{ml}$ einer 1 Molaren Schwefelsäure versetzt, 30 min geschüttelt und die Natriumkonzentrationen am ICP bestimmt.

\subsubsection{Frachtabschätzung}

Zur Abschätzung der durch Niederschläge eingetragenen lonenfracht wurden mittlere und hohe Konzentrationen aus Niederschlagsuntersuchungen in Bulili (Nicklas 2006) herangezogen und Ladungsbezogen auf Na-lonen berechnet. Die berechnete Menge von $500 \mathrm{mg}$ Kochsalz wurde in 5 I Wasser gelöst und durch mit 25 g gefüllten IER-Kollektoren perkoliert. Der Versuch wurde an drei 
Wiederholungen durchgeführt und die Konzentrationen der aufgegebenen und ablaufenden Lösung am ICP bestimmt.

\subsubsection{Fixierungs- und Perkolationsversuche}

Niederschlagswässer sind Mischungen mehrerer Elemente und Nährstoffe sowie Schadstoffe in unterschiedlichen Konzentrationen. Die Konzentrationen der Elemente und Nährstoffe unterliegen einer starken Schwankung im Regenwasser und werden während der anhaltenden Niederschlagsdauer durch Verdünnung geringer.

Um die Eignung des Ionentauschers zur Fixierung verschiedener Ionen unterschiedlicher Konzentrationsbereiche sowie die Bindungseigenschaften der lonen am Ionentauscher zu überprüfen, wurde eine hochkonzentrierte Kochsalzlösung mit $850 \mathrm{mg} \mathrm{NaCl}$ in 5 I gelöst sowie jeweils 5 I unterschiedlich konzentrierte Multielementlösungen mit den Elementen $\mathrm{Al}, \mathrm{Ca}, \mathrm{Fe}, \mathrm{K}, \mathrm{Mg}, \mathrm{Mn}$ und $\mathrm{Na}$ durch die IER-Kollektoren innerhalb von 4 Stunden perkoliert. Von den Lösungen wurden vor und nach der Perkolation Proben genommen und der Grad des lonenentzuges berechnet.

Um Informationen zur Remobilisierung der fixierten Ionen zu erhalten wurde die lonentauscherfüllung der Sammelröhren wie oben beschreiben zerlegt und extrahiert. Die Wiederholungen der IER-Kollektoren wurden mit Sammler 1 bis Sammler 3, die Schichten der IERKollektoren mit Schicht 1-5 bezeichnet, wobei Schicht 1 für die oberste Schicht und Schicht 5 für die unterste (dem Ablauf nahen) Schicht steht.

\subsubsection{Blindwerte}

Zur Bestimmung der Blindwerte des lonentauschermaterials wurden Blindwertläufe aus den Fixierund Perkolationsversuchen, der Kapazitätsbestimmung als auch der Batchexperimente entsprechend aufgearbeitet.

\subsubsection{Fließgeschwindigkeiten}

Überprüft wurde, ob Starkregenereignisse mit hohen Fließgeschwindigkeiten im IER-Kollektor einen Einfluss auf die Fixierleistung der IER-Kollektoren zur Folge haben. In Variante 1 wurden drei IERKollektoren mit senkrechtem Fixierschlauch aufgebaut.

In Variante 2 wurden drei IER-Kollektoren knapp über der IER- Füllung abgeknickt und der Schlauch mit der IER-Füllung senkrecht nach oben gebogen, so dass ein U-förmiger Schlauch entstand. Durch die Verengung des Querschnitts und der entstehenden Wassersäule wurde perkolierendes Wasser stark gebremst und damit die Verweil- und Reaktionszeit im Sammler erhöht.

Als dritte Versuchsvariante wurden 3 Netze mit jeweils $50 \mathrm{~g}$ lonentauschern (IER-Kugeln) befüllt und für 12 Stunden ohne Schüttelbewegung (entsprechend einem Auffangkanister im Gelände) in die zu untersuchende Lösung gebracht. Bei dieser Variante wurde davon ausgegangen, dass anfallendes Regenwasser in einem ausreichend großen Behältnis gesammelt wird.

In Variante 1 und 2 wurden zur Simulation eines Starkregenereignisses 2 I einer Multielementlösung innerhalb von 14 min durch die geknickten und innerhalb von 8 min und 9 min durch die gestreckten IER-Kollektoren perkoliert. Die Lösungsmenge, bezogen auf die Trichterfläche, entspricht 
Regenereignissen rund $5 \mathrm{~mm}-8 \mathrm{~mm}$ pro min oder $300-478 \mathrm{~mm}$ pro Stunde. Regenereignisse in diesen Dimensionen sind auch in tropischen Regionen selten bis auszuschließen. Die Ionentauscherfüllung der IER-Kollektoren wurde wie beschrieben aufgearbeitet. Aus dem Ionentauscher der Beutel wurde ein Adäquat eingewogen und mit $50 \mathrm{ml} 1$ molarer Schwefelsäure versetzt. Die Extraktion erfolgte in dreimaliger Wiederholung.

Zusätzlich wurden Proben der Aufgabelösung sowie vom Perkolat genommen.

\subsubsection{Verdünnungs- und Durchwaschungsversuch}

Während des Verlaufs eines Niederschlagsereignisses nehmen Konzentrationen im Niederschlagswasser ab. In diesem Versuch sollte untersucht werden, ob eine stetige Verdünnung zu einer verminderten Fixierleistung oder gar einer Mobilisierung der lonen auf das Ionentauschermaterial zur Folge haben. Dabei wurden an 3 IER-Kollektoren jeweils 2 I einer Multielementlösung mit $5 \mathrm{~g}$ Totalgehalt der zu untersuchenden Elemente angesetzt, davon $1 \mathrm{I}$ auf den IER-Kollektor gegeben und der Messkolben erneut auf 2 I aufgefüllt. Insgesamt wurden 5 Verdünnungsschritte an der Lösung durchgeführt. Zur Kontrolle wurde eine konstant konzentrierte Lösung durch 3 IER-Kollektoren perkoliert. Der Versuch wurden blindwertbereinigt.

\subsection{Ergebnisse der Laborversuche}

\subsubsection{Kapazitätsbestimmung des lonentauschers}

Bei der Bestimmung der Fixierungskapazität wurde an drei Wiederholungen eine mittlere Ladungskapazität von $0,5 \mathrm{mmol}_{\mathrm{c}} \mathrm{g}^{-1}$ und einer Standartabweichung von $0,07 \mathrm{mmol}_{\mathrm{c}} \mathrm{g}^{-1}$ ermittelt. Die weite Streuung der Standardabweichung ergab sich aus der geringen Wiederholung und der kleinen Ionentauschereinwaage. Die Kapazitätsbestimmung diente einer Überprüfung der Herstellerangaben, um die einzusetzende Menge im Feld abschätzen zu können.

\subsubsection{Frachtabschätzung}

Im Versuch zur Abschätzung der eingetragenen Nährstofffrachten, bei dem Frachtmengen nach Nicklas (2006) in Form einer entsprechend berechneten Kochsalzlösung ( $\mathrm{NaCl}$-Lösung) appliziert wurden, konnten im Mittel 96\% (Tab. 2.1) der lonenfracht aus der Lösung am lonentauscher (25 g) fixiert werden. Dabei wurden 69\% der Ladungskapazität des eingesetzten Tauschers belegt.

Tab. 2.1: Fixierungsleistung und Belegungsanteile einer Freilandfrachtabschätzung 
2 Laborexperimente

\begin{tabular}{|c|c|c|c|c|c|}
\hline & & Sammler 1 & Sammler & ammler & \\
\hline Na aufgegeben & $\mathrm{mg}$ & 196.0 & 212.7 & 206.1 & \\
\hline Na ablaufende Lösung & $\mathrm{mg}$ & 17.9 & 3.0 & 2.3 & \\
\hline Na im IER-KolleKtor fixiert & $\mathrm{mg}$ & 178.1 & 209.7 & 203.7 & Mittelwerte \\
\hline Entzug aus der Lösung & $\%$ & 90.9 & 98.6 & 98.9 & 96 \\
\hline IER-KolleKtorbelegung & $\mathrm{mmol}_{\mathrm{c}}$ & 7.74 & 9.12 & 8.86 & 8.57 \\
\hline Belegung der Ladungskapatzität & $\%$ & 61.9 & 72.9 & 70.9 & 69 \\
\hline
\end{tabular}

Im der Wiederholung 1 wurden die Lösungen schneller Perkoliert als in den Wiederholungen 2 und 3. Hier deutet sich bereits an, dass eine schnellere Perkolation von Lösungen durch die IER-Kollektoren zu einer verminderten Sorption am Tauscher führt. Der lonenentzug aus der Lösung lag zwischen 90 und $98 \%$ trotz der hohen lonenstärke in der perkolierten Lösung. Die langsamer perkolierten Lösungen belegen über $70 \%$ der Ladungsplätze. Um bei erhöhten und extremen Ereignissen noch genügend Ladungsplätze zur Verfügung zu haben sowie den Perkolationsweg zu verlängern, wurden alle weiteren Versuche mit $50 \mathrm{~g}$ lonentauscher durchgeführt.

\subsubsection{Fixierungs- und Perkolationsversuche}

Zur Beurteilung der Effizienz des Rücktausches (Extraktion) wurden die extrahierten Mengen an $\mathrm{Na}$ der berechneten Verarmung in der Lösung gegenüber gestellt.

In Tab. 2.2 sind die summierten Gesamtgehalte aus der Extraktion sowie die Differenzen der Aufgabelösungen zur Ablauflösung und die prozentuale Wiederfindung dargestellt. Im Mittel werden $100 \%$ des Na wiedergefunden. Zwei Werte liegen jedoch über 100\%, was auf Blindwerte im Ionentauscher und Hintergrundwerte aus dem Labor zurück geführt wird.

Tab. 2.2: Gesamtmengen und deren Bilanz aus den Extraktions- und Perkolationsuntersuchungen

\begin{tabular}{c|ccc} 
& $\begin{array}{c}\text { Verarmung der } \\
\text { Perkolationslösung } \\
\text { mg }\end{array}$ & $\begin{array}{c}\text { Extraktionsmenge } \\
\text { mg }\end{array}$ & Wiederfindung \\
\hline Sammler 1 & 311,54 & 330,98 & 106 \\
Sammler 2 & 292,27 & 272,92 & 93 \\
Sammler 3 & 322,22 & 329,52 & 102
\end{tabular}

Zur Beurteilung des Sorptionsortes in den IER-Kollektoren werden die in Tab. 2.3 dargestellten Summen in Abb. 2.3 schichtweise gezeigt. 


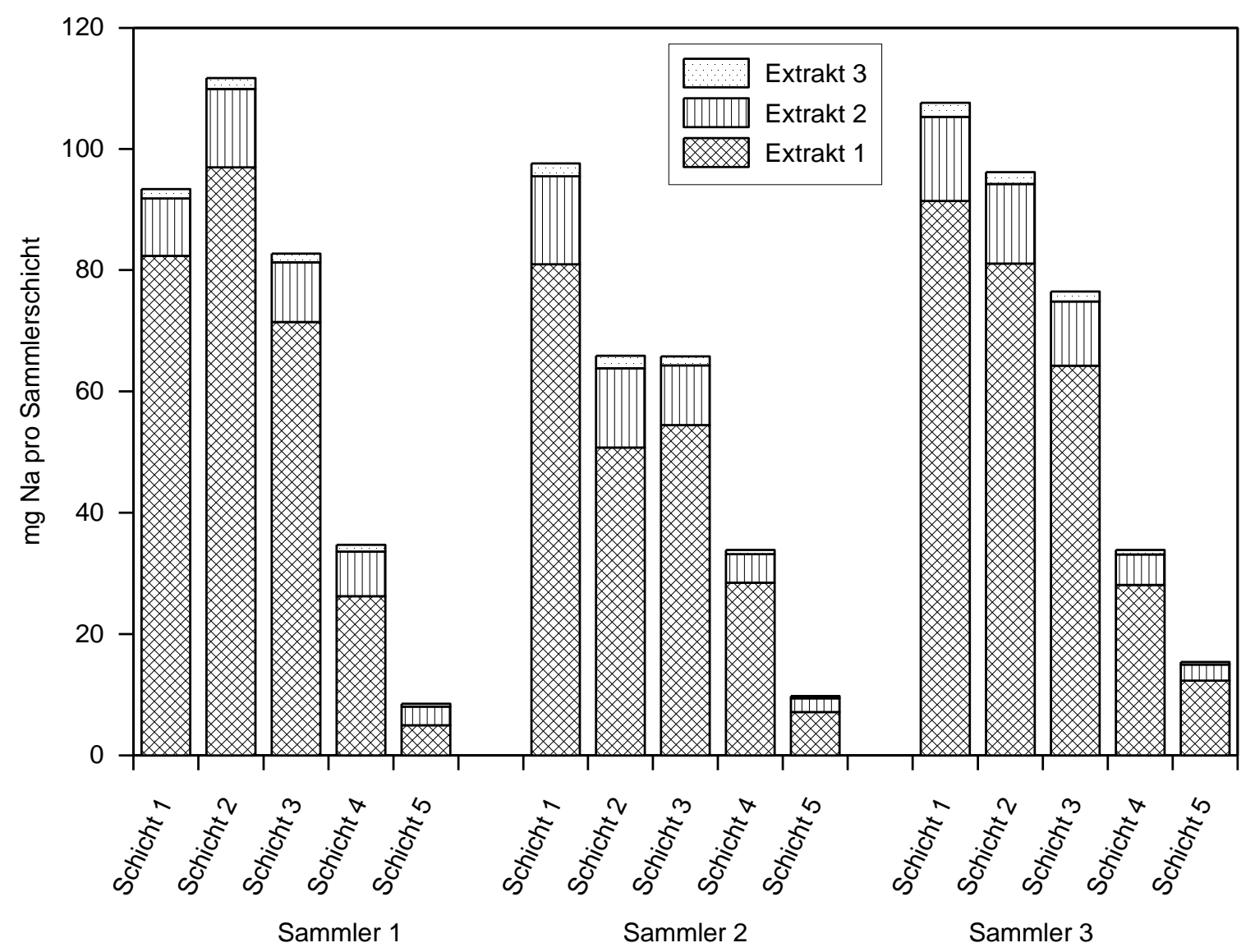

Abb. 2.2: Akkumulationsprofile in den IER-Kollektoren für Natrium

Die Schichtabfolge in den IER-Kollektoren zeigt, dass die bevorzugte Fixierung in den oberen Schichten 1 bis 3 erfolgt. Deutlich zu erkennen ist, dass der höchste Anteil der fixierten lonen im ersten Extraktionsschritt mit 75,9\% - 90,5\% erfasst wird, während im zweiten Extraktionsschritt 13,7\% - 15,2\% und im dritten Extraktionsschritt 2,0\% - 2,3\% Na-Beladung extrahiert werden. Bezogen wurden die Angaben auf die aus dem Lösungsentzug stammende Menge. Die unterschiedliche Fixierleistung der 1. und 2. Schichten, besonders in der Wiederholung 1, ist eine Folge unterschiedlicher Schichtmassen bei der Probenaufarbeitung.

\subsubsection{Perkolationsversuche mit Multielementlösungen}

Bei den dargestellten Akkumulationen in IER-Kollektor handelt es sich um typische Profile in den IERKollektoren, wie sie für alle Nährstoffe, unabhängig der eingesetzten Konzentrationen, gefunden wurden. Exemplarisch sind in Abb. 2.3 die Akkumulationsprofile für die Elemente $\mathrm{Al}, \mathrm{Fe}$ und $\mathrm{Mn}$ gezeigt. Es zeigt sich, dass die Akkumulation der Ionen Hauptsächlich in den Schichten 1 bis 3 $(0-6 \mathrm{~cm})$ stattfindet. Die Schichten 4 und 5 würden im Feldeinsatz als Fixierpuffer für unerwartet hohe Einträge fungieren.

Für alle 7 untersuchten Elemente wurden in den Schichten 4 und 5 geringe Gehalte der entsprechenden Ionen gefunden. Eine Überprüfung ergab, dass die Gehalte im Bereich der Blindwerte liegen. 


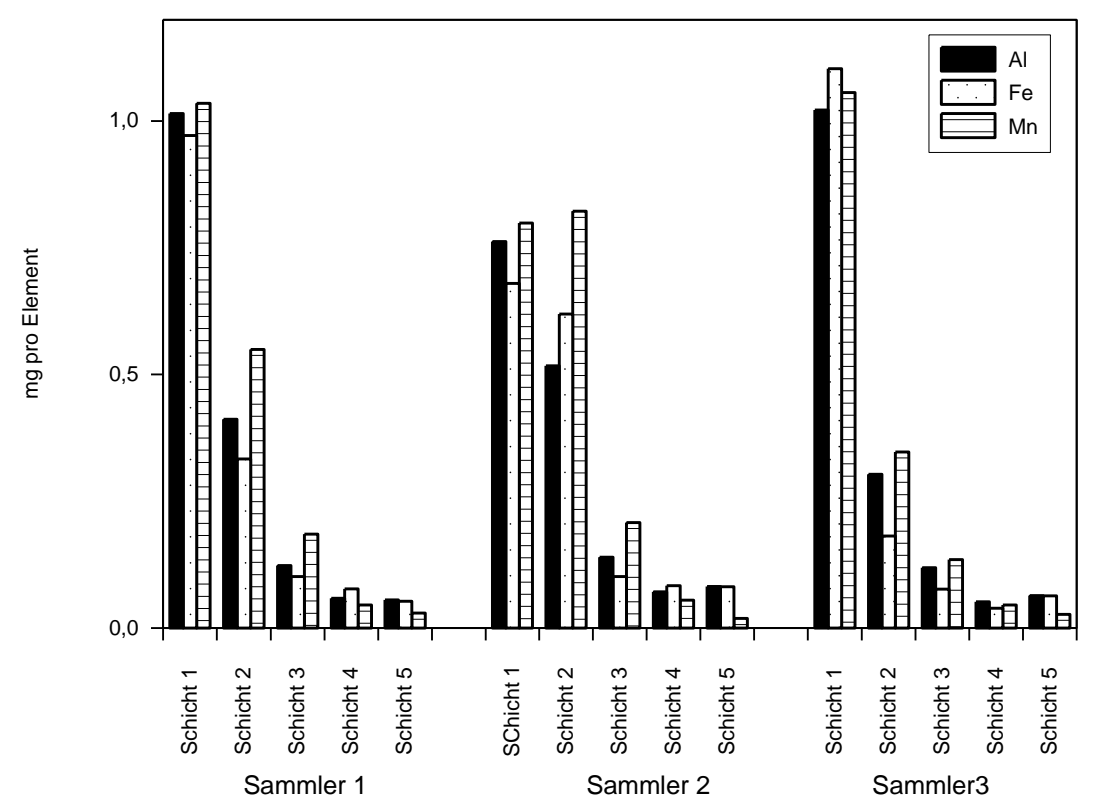

Abb. 2.3: Akkumulationsprofile für die Elemente $\mathrm{Al}, \mathrm{Fe}$ und $\mathrm{Mn}$

Die fixierten Gehalte unterscheiden sich elementabhängig. Gut fixiert, mit geringen Standardabweichungen, werden $\mathrm{Al}, \mathrm{Mn}, \mathrm{P}$ und $\mathrm{NO}_{3}-\mathrm{N}$ mit Entzügen aus den Lösungen von über $95 \%$. Mit geringeren Fixierungsleistung sind die Elemente $\mathrm{Ca}$ und $\mathrm{Fe}$ mit knapp 80\% Fixierung zu verzeichnen. Bei diesen Elementen zeigen die hohen Standardabweichungen eine gewisse Unschärfe im Ergebnis und sind konzentrationsabhängig. Die geringste Fixierleistung zeigte Na mit $84 \%$. Zu beachten ist, dass bei den Elementen $\mathrm{K}$ und $\mathrm{Na}$ die eingangs erwähnten Versuche mit sehr hohen Konzentrationen nicht berücksichtigt wurden, da aufgrund der hohen lonensättigung als Monoelementlösung keine Konkurrenz am lonentauschervorliegt und das Ergebnis zwangsläufig verbessern würden.

Tab. 2.3: Lösungsentzug der Elementionen

\begin{tabular}{l|ccccc} 
& $\begin{array}{c}\text { Mittelwert des } \\
\text { Elemententzug }\end{array}$ & $\begin{array}{c}\text { Standard- } \\
\text { abweichung }\end{array}$ & $\begin{array}{c}\text { Anzahl Prüf- } \\
\text { lösungen }\end{array}$ & $\begin{array}{c}\text { untere } \\
\text { Konzentration }\end{array}$ & $\begin{array}{c}\text { obere } \\
\text { Konzentration }\end{array}$ \\
\hline $\mathrm{Al}$ & $\%$ & & & $\mathrm{mg}^{*} \mathrm{l}^{-1}$ & $\mathrm{mg}^{*} \mathrm{l}^{-1}$ \\
$\mathrm{Ca}$ & 96.7 & 1.90 & $\mathrm{n}=13$ & 0.5 & 2.9 \\
$\mathrm{Fe}$ & 79.2 & 11.14 & $\mathrm{n}=13$ & 0.6 & 4.1 \\
$\mathrm{~K}$ & 79.5 & 9.04 & $\mathrm{n}=12$ & 0.1 & 2.8 \\
$\mathrm{Mg}$ & 91.4 & 6.28 & $\mathrm{n}=18$ & 0.2 & 3.0 \\
$\mathrm{Mn}$ & 87.4 & 6.96 & $\mathrm{n}=19$ & 0.2 & 3.5 \\
$\mathrm{Na}$ & 98.3 & 1.20 & $\mathrm{n}=12$ & 0.5 & 3.0 \\
$\mathrm{P}$ & 84.3 & 11.83 & $\mathrm{n}=19$ & 0.8 & 62.5 \\
$\mathrm{NO}_{3}-\mathrm{N}$ & 99.7 & 1.1 & $\mathrm{n}=18$ & 1.0 & 50.0 \\
& 99.7 & 0.7 & $\mathrm{n}=18$ & 1.0 & 50.0
\end{tabular}

Die mittleren Elemententzüge verschiedener Lösungen unterschiedlicher Konzentrationsbereiche mit unterer und oberer Konzentrationsgrenze werden in Tab. 2.3 zusammen gefasst. Die Ergebnisse zeigen, dass ein hoher bis nahezu vollständiger Entzug von lonen aus Niederschlägen mittels IERKollektoren möglich ist. 
Um aus den Extrakten der IER-Kollektoren auf den ursprünglichen Gesamteintrag schließen zu können, sind Kenntnisse zur Remobilisierung der Elemente aus den Ionentauschern mittels Extraktion notwendig. Zum Vergleich wurden die Gehalte in den Extrakten auf die Gehalte der Ausgangslösungen bezogen.

Hohe Wiederfindungsraten ergaben sich für einwertige und mobile lonen wie $\mathrm{K}$ und $\mathrm{Na}$ mit $92 \%$ sowie Nitrat-Stickstoff $\left(\mathrm{NO}_{3}-\mathrm{N}\right)$ mit 105\%. Geringere Extraktionswiederfindungen zwischen $47 \%$ und $80 \%$ wurden für die verbleibenden Elemente ermittelt (Tab. 2.4). Es zeigt sich, dass einwertig gebundene Elemente leichter remobilisiert werden können und mit zunehmender Ladungsstärke und kleineren Atomdurchmessern die Bindungsstärke steigt. Die Massendifferenz zwischen Lösungsentzug und Remobilisierung durch Extraktion muss auf dem Ionentauscher verblieben sein und ist mit $0,2 \%$ bis $32 \%$ elementspezifisch (Tab. 2.4). Bei den Elementen $\mathrm{Na}$ und $\mathrm{NO}_{3}-\mathrm{N}$ wurden leichte Massenzugewinne verzeichnet. Negative Differenzen werden auf Kontaminationen zurückgeführt und sind in der Literatur für diese Elemente bekannt (Crabtree and Trudgill 1981).

Die Wiederfindungsraten wurden herangezogen, um Faktoren für die Korrektur der Freilandproben zu berechnen (Tab. 2.4), da die Extraktionszeit und der entsprechende Aufwand begrenzt waren. Die Werte wurden im Vorgriff um die Blindwerte aus den lonentauscher bereinigt. Um bei den ermittelten Gehalten auf den Ausgangsgehalt schließen zu können, wurden nährstoffspezifische Korrekturfaktoren berechnet (Tab. 2.4).

Tab. 2.4: Gehaltswiederfindung in Prozent in den Extrakten der IER-Kollektoren

\begin{tabular}{l|cccccccc} 
& $\mathrm{Al}$ & $\mathrm{Ca}$ & $\mathrm{Fe}$ & $\mathrm{K}$ & $\mathrm{Mg}$ & $\mathrm{Mn}$ & $\mathrm{Na}$ & $\mathrm{NO}_{3}-\mathrm{N}$ \\
\hline Wiederfindung im Extrakt \% & 64.2 & 47.1 & 65.2 & 91.9 & 62.4 & 80.2 & 91.7 & 105.1 \\
Standardabweichung & 5.42 & 4.11 & 16.85 & 17.27 & 5.57 & 8.28 & 8.76 & 16.9 \\
Stichprobenumfang & $\mathrm{n}=9$ & $\mathrm{n}=6$ & $\mathrm{n}=8$ & $\mathrm{n}=9$ & $\mathrm{n}=9$ & $\mathrm{n}=9$ & $\mathrm{n}=10$ & $\mathrm{n}=9$ \\
& & & & & & & & \\
Differenz zum Lösungsentzug \% & 32.5 & 32.1 & 14.3 & 0.2 & 25.0 & 18.1 & -7.4 & -5.4 \\
Korrekturfaktor (Prüflösungsgehalt) & 1.558 & 2.121 & 1.533 & 1.089 & 1.602 & 1.247 & 1.091 & 0.952
\end{tabular}

\subsubsection{Blindwerte}

Die Bestimmung der Blindwerte zeigt aufgrund der geringen Gehalte und der Schwankungen zwischen den Chargen des lonentauscher hohe Standardabweichungen besonders bei den Elementen $\mathrm{K}, \mathrm{Mg}, \mathrm{Ca}$ und $\mathrm{NO}_{3}-\mathrm{N}$. Die höchsten Blindwerte wurden für die Elemente $\mathrm{Ca}, \mathrm{Mg}$ und $\mathrm{K}$ erfasst. Ein Vergleich mit den im Freiland monatlich akkumulierten Mengen ergab, dass die Blindwerte einen vernachlässigbaren Einfluss auf das Depositionsergebnis ausüben. Anhand der Abschätzung wurden Korrekturen an den Freilandproben durchgeführt. Die Blindwerte sind in 
Tab. 2.5 aufgeführt.

Tab. 2.5: Blindwerte aus den Extrakten des lonentauschers

\begin{tabular}{l|ccc} 
& $\begin{array}{c}\text { mg pro g } \\
\text { lonentauscher }\end{array}$ & $\begin{array}{c}\text { Standart- } \\
\text { abweichung }\end{array}$ & $\begin{array}{c}\text { Versuchs- } \\
\text { wiederholung }\end{array}$ \\
\hline $\mathrm{Al}$ & 0.0018 & 0.0011 & $\mathrm{n}=13$ \\
$\mathrm{Ca}$ & 0.0138 & 0.0184 & $\mathrm{n}=13$ \\
$\mathrm{Fe}$ & 0.0015 & 0.0010 & $\mathrm{n}=13$ \\
$\mathrm{~K}$ & 0.0223 & 0.0395 & $\mathrm{n}=13$ \\
$\mathrm{Mg}$ & 0.0301 & 0.0530 & $\mathrm{n}=13$ \\
$\mathrm{Mn}$ & 0.0010 & 0.0012 & $\mathrm{n}=13$ \\
$\mathrm{Na}$ & 0.0043 & 0.0058 & $\mathrm{n}=10$ \\
$\mathrm{P}$ & 0.0018 & 0.0012 & $\mathrm{n}=7$ \\
$\mathrm{NO3-N}$ & 0.0032 & 0.0143 & $\mathrm{n}=15$
\end{tabular}

\subsubsection{Fließgeschwindigkeiten}

Zur Simulation hoher Niederschlagsereignisse wurden unterschiedlichen Konstruktionen der IERKollektoren entwickelt. Es wurde dabei nur eine Auswahl an Elementen berücksichtigt, da grundlegende Untersuchungen zur Fixierung bereits zuvor (Kapitel 2.3.3) durchgeführt wurden. Die Differenz zwischen Aufgabe und Ablauflösung mit einer Perkolationsgeschwindigkeit von über $300 \mathrm{~mm}$ pro Stunde zeigte für die gestreckten Röhren Elementverarmungen im Bereich von $83 \%$ bis $97 \%$ (Tab. 2.6). Na liegt mit 76\% deutlich unter diesem Bereich. Die Elemententzüge sind etwas geringer als die angegebenen mittleren oben angeführten Entzüge der Perkolationsversuche mit Multielementlösungen, jedoch noch im Bereich der Standardabweichung.

Tab. 2.6: Prozentuale Verarmung varianten an IER-Kollektoren zum Nährstoffentzug bei hohen Perkolationsgeschwindigkeiten

\begin{tabular}{l|cccccccc} 
& Elemententzug & Al & $\mathrm{Ca}$ & $\mathrm{Fe}$ & $\mathrm{K}$ & $\mathrm{Mg}$ & $\mathrm{Mn}$ & $\mathrm{Na}$ \\
\hline IER gestreckt & $\%$ & 95 & 83 & 79 & 95 & 90 & 97 & 76 \\
IER-geknickt & $\%$ & 97 & 85 & 84 & 95 & 93 & 99 & 82 \\
IER-Kugel & $\%$ & 26 & 27 & 35 & 41 & 29 & 26 & 39
\end{tabular}

Bei der Variante "IER-Kollektor geknickt" ist durch die Verringerung der Perkolationsgeschwindigkeit ein mittlerer Anstieg der Fixierleistung von 3\% zu verzeichnen. Mit 82\% Entzug stellt Na die untere Grenze dar, die bei den geknickten Röhren um 6\% höher liegt als bei den gestreckten Röhren. Bei dem Element $\mathrm{Mn}$ wird ein Entzug von 99\% erreicht und stellt eine Verbesserung um 2\% dar.

Bei der Fixierung der Elemente durch IER-Kugeln in der Lösung wurden Entzugsraten von 25\% bis 41\% (Tab. 2.6) gefunden und damit in der Effizienz des Entzugs als sehr gering bewertet.

\subsubsection{Verdünnungs- und Durchwaschungsversuch}

IER-Kollektoren, die mit einer sich verdünnenden Lösung (nach 50\% Aufgabe der Lösung wurde die Lösung auf das Ursprüngliche Volumen erneut aufgefüllt), und damit mit einer deutlich höheren Perkolationsmenge beschickt wurden, zeigten höhere Akkumulationen an Nährstoffen. Fixierleistungen lagen im Bereich von $61 \%$ bis $100 \%$. IER-Kollektoren, die mit einer konstant 
konzentrierten Lösung (Tab. 2.7) beladen wurden, zeigten im Mittel eine 8\% geringere Fixierleistung. Beide Lösungen zeigten etwas schlechtere Fixierleistungen als im obengenannten Versuch, was auf schnellere Perkolation zurück geführt wird.

Tab. 2.7: Berechneter Lösungsentzug der Lösungen im Verdünnungsversuch in \%

\begin{tabular}{l|cccccccc} 
& $\mathrm{Al}$ & $\mathrm{Ca}$ & $\mathrm{Fe}$ & $\mathrm{K}$ & $\mathrm{Mg}$ & $\mathrm{Mn}$ & $\mathrm{Na}$ & $\mathrm{P}$ \\
\hline Lösung mit kleiner werdender Konzentration & 90.1 & 91.7 & 81.2 & 61.1 & 74.5 & 80.0 & 100.4 & 64.4 \\
Lösung konstnater Konzentration & 77.5 & 81.6 & 80.2 & 54.5 & 71.7 & 75.5 & 77.8 & 59.6
\end{tabular}

Durch die hohe Menge an aufgegebener Perkolationslösung wurden Effekte einer Verlagerung im IER-Kollektor vermutet. Diese konnten aufgrund der Fixierungsleistung der Schichten im IER-Kollektor ausgeschlossen werden. Exemplarisch werden die Elemente Al, Ca und Mn dargestellt (Abb. 2.4).

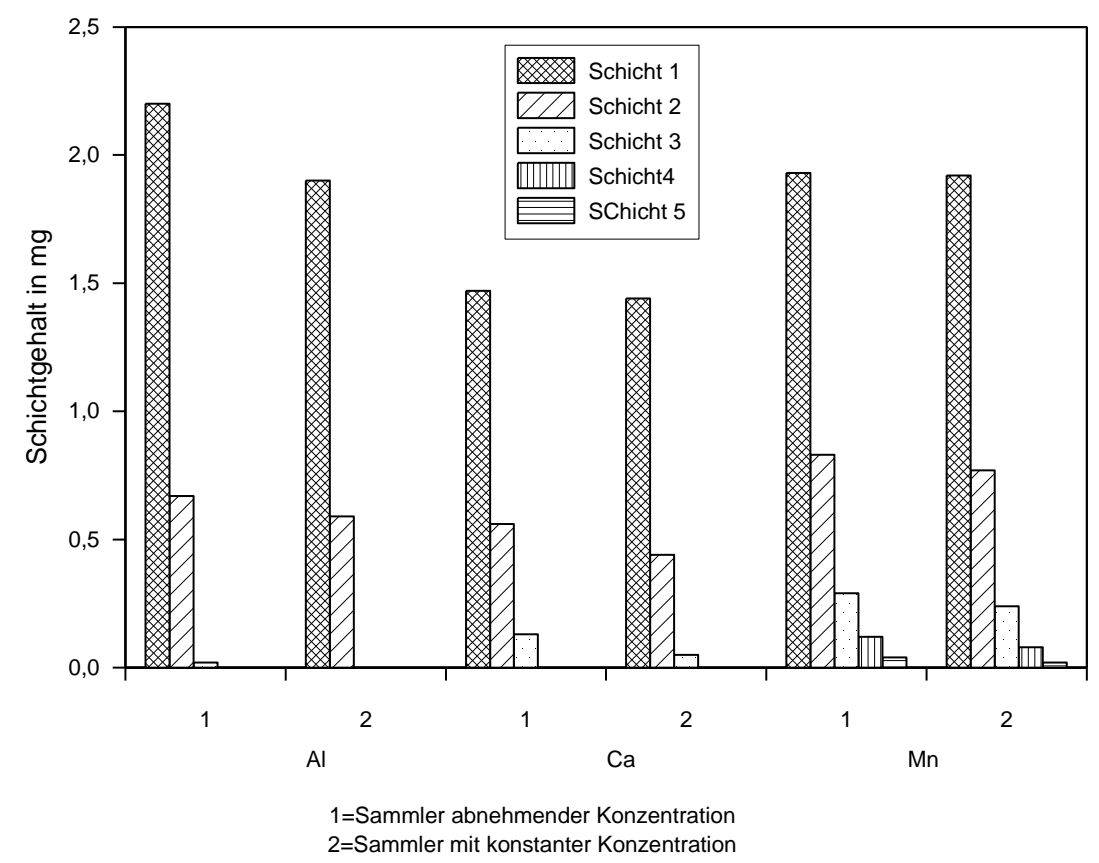

Abb. 2.4: Mittelwerte der Schichtbeladung in den Varianten des Verdünnungsversuches

Bei der Verdünnungsvariante mit abnehmenden Konzentrationen zeigten sich leicht höhere Fixieranteile in den 1. Schichten gegenüber den Vergleichssammlern. Dieser Trend setzt sich bis in den unteren Teil der IER-Kollektoren fort. Die verbleibende Ladungskapazität wird in verdünnten Lösungen stärker genutzt.

Elementgehalte aus sich verdünnenden Lösungen mit geringen Konzentrationen konnten trotz der hohen Perkolationsgeschwindigkeiten besser fixiert werden als Elementgehalte konstant konzentrierter Lösungen. Es wurde gezeigt, dass abnehmende Konzentrationen im Regenwasser durch IER-Kollektoren sicher erfasst werden können.

\subsection{Diskussion}

Im Laborexperiment wurden die IER-Kollektoren unter Extrembedingungen getestet. Die Versuche mit Fließgeschwindigkeiten und Konzentrationen, die über den Bedingungen im Freiland liegen, 
wurden durchgeführt, um Schwächen des Systems aufzuzeigen. Allen Berechnungen liegen Depositionsfrachten zugrunde, die im Untersuchungsgebiet von Nicklas (2006) erhoben wurden. Mit einer mittleren Ladungskapazität von $0,5 \mathrm{mmol}_{\mathrm{c}} \mathrm{g}^{-1}$ entspricht der lonentauscher den Angaben des Herstellers und stellt ausreichend dichte Ladungsplätze zu Verfügung. In allen Laborergebnissen wurde kein lonendurchbruch aufgrund mangelnder Ladungsplätze registriert.

Die nach Niklas (2006) berechneten monatlichen Frachten lagen in Bereichen zwischen 8 mmol $_{c}$. und $15 \mathrm{mmol}_{\mathrm{c}}$. Bei den eingesetzten $25 \mathrm{~g}$ lonentauscher wurden nahezu 70\% der Ladungsplätze belegt (Tab. 2.1). Durch die Berechnung der Depositionseinträge mittels klassischer Niederschlagssammeltechnik und damit aufgrund geringer Konzentrationen wurde eine Unterschätzung der berechneten Nährstoffmengen vermutet. Um einen erhöhten Nährstoffeintrag ausreichende Pufferkapazitäten bereitzustellen, wurde die lonentauschermenge auf $50 \mathrm{~g}$ erhöht und damit die Ladungskapazität verdoppelt. Rechnerich ergeben die Ergebnisse von Niklas (2006), die im Untersuchungsgebiet durchgeführt wurden, eine Belegung der Kapazität zwischen 32\% und 60\%. Damit ist ausreichend Puffer für Nährstoffeinträge in Freiland vorhanden.

Die Verteilung der Nährstoffe im IER-Kollektor ergab, dass Nährstoffe bevorzugt in den oberen Schichten zwischen $0 \mathrm{~cm}$ und $6 \mathrm{~cm}$ sorbiert werden (Abb. 2.3). Mit einem mittleren Entzug von $96 \%$ (Tab. 2.2) bzw. 100\% (Tab. 2.3) der Na-lonen aus der Lösung konnte gezeigt werden, dass der IERKollektor prinzipiell für die Erfassung von Nährstoffen in Niederschlagswässern geeignet ist. Kritisch wurden jedoch die Einzelwerte mit 102\% und 106\% Wiederfindung in Tab. 2.2 betrachtet. Die Werte werden als Kontaminationsartefakte gewertet. Crabtree und Trudgill (1981) berichten für das Element $\mathrm{Na}$ ebenfalls eine Wiederfindung von 105\%. Andere Elemente zeigen in diesem Artikel Minderbefunde. Simkin et al (2004) findet bei geringen Konzentrationen für Nitrat Werte grösser $100 \%$.

Die anionischen Stoffe wie Nitrat oder Phosphat werden in unserem Versuch mit nahezu $100 \%$ am Ionentauscher festgelegt (Tab. 2.3). Ursache hierfür ist einerseits die höhere Konzentration in den Lösungen und die geringere Konkurrenz der Anionen am lonentauscher, da viele der Kationen als Nitratverbindung vorgelegt wurden. In den Untersuchungen von Simkin et al (2004) wurde bei den Anionen Sulfat, Nitrat und Chlor mit steigenden Gehalten eine Verminderung der Wiederfindung beobachtet. Jedoch sind Wiederfindungen im Bereich zwischen $94 \%$ und $100 \%$ als hoch anzusehen. Durch die angelagerten Sauerstoffmoleküle in den Anionenmolekülen kommt es zu einer starken Polarisation der Moleküle die sich leicht an den freien Ladungsplätzen der lonentauscher binden lassen. Aufgrund der Molekülgröße können diese Bindungen bei der Extraktion wiederum leicht gelöst werden.

Kationische Frachten zeigen in der Fixierungsleistung einen deutlich weiteren Bereich (Tab. 2.3). Der Entzug aus Lösungen liegt bei knapp 80\% für Fe und Ca bis 98\% für Mn. Als Ursachen werden hierfür physikalische Randbedingungen wie Ionendurchmesser, Ladung der lonen sowie Wechselwirkungen und Anlagerungen von Wassermolekülen um die lonen angenommen. Zusammengefasst kann dies als Konkurrenz um freie Landungsplätze aufgefasst werden. Die Konkurrenz tritt deutlich beim Element $\mathrm{Na}$, einem im Durchmesser großen Element mit einfacher Ladung zum Vorschein. Zum Beispiel wird $\mathrm{Na}$ in vorrangegangenen Monoelementlösungen mit $96 \%$ festgelegt. Deutlich geringer 
ist der Entzug aus Multielementlösungen mit 84\% im Mittel und zeigt eine deutlich geringeren Entzug aus den Lösungen, als das kleinere einfachgeladene Alkalielement K (Tab. 2.3). In der Verteilung innerhalb des IER-Kollektors unterscheiden sich die Elemente jedoch nicht. Eine bevorzugte Festlegung erfolgt in der oberen Schicht durch die Absättigung der freien Ladungsplätze.

Die von Nicklas (2006) berechneten mittleren Konzentrationen über alle Messzeiträume sind gering und fallen in den unteren Erfassungsbereich vieler Messgeräte. Dabei können diese dementsprechend nur unscharf oder gar nicht erfasst werden. Besonders deutlich wird dies für die Elemente $\mathrm{Al}$, Fe und besonders für $\mathrm{Mn}$, die mit Konzentrationen $<0,1 \mathrm{mg}^{-1}$ bei Niklas (2006) angegeben werden. Von ähnlichen Problemen Berichtet Gutzler (2011) in seiner Arbeit und gibt für die Nährstoffe $\mathrm{NO}_{3}-\mathrm{N}$ und $\mathrm{P}$ aufgrund der geringen Konzentrationen einen unteren und oberen Depositionsbereich an. Nährstoffe wie $\mathrm{Al}, \mathrm{Fe}$ und $\mathrm{Mn}$ konnten aufgrund der geringen Konzentrationen im Niederschlag nicht bilanziert werden. Die Erfassung geringer Konzentrationen bereitet vor allem für standardmäßig ausgerüstete oder ältere Laboreinheiten Probleme, wie sie in den Tropen häufig anzutreffen sind. Für spezialisierte Einrichtungen, die sich auf Wasseranalytik im geringen Konzentrationsbereich spezialisiert haben (Graphitrohr AAS), können auch Konzentrationen deutlich unter diesen Bereichen erfasst werden.

Durch die Akkumulation der Nährstoffe und der Extraktion mit einer geringen Menge an Extraktionsmittel wurde eine Erhöhung der Konzentration im Extrakt erreicht und die analytische Bestimmung in einen Bereich der besseren Detektierbarkeit verlegt. Berechnete Konzentrationen der Depositionselemente von Nicklas (2006) ergaben im Extrakt Konzentrationen für Makroelemente zwischen $1 \mathrm{mg} \mathrm{I}^{-1}$ und $25 \mathrm{mg} \mathrm{I}^{-1}$. Für Elemente wie Fe oder Al wurden Konzentrationen zwischen $0,4 \mathrm{mg} \mathrm{l}^{-1}$ und $1 \mathrm{mg} \mathrm{I}^{-1}$ berechnet. Einzig für Mn konnte durch Anreicherung der Depositionsfracht keine Konzentrationsanhebung über $0,1 \mathrm{mg} \mathrm{I}^{-1}$ berechnet werden. Die hier vorgestellten berechneten Werte wurden in weiteren Untersuchungen im Freiland gefunden und zum Teil übertroffen, da bei der Berechnung die überwiegende Fixierung in der oberen Schicht vernachlässigt wurde. In der Literatur werden Konzentrationssteigerung im Extrakt nicht berichtet, da Faktoren wie zum Beispiel Extraktionszeit, Stärke und Art des Extraktionsmittels und andere die Extraktion maßgeblich bestimmen. Angegeben werden flächenbezogene Depositionsmengen.

Aufgrund der unvollständigen Extraktion ist selbst bei hohen Fixierleistungen im lonentauscher eine geringere Wiederfindung unumgänglich. Die in dieser Untersuchung gefundenen Wiederfindungen im Extrakt liegen zwischen 60\% und 90\% der ursprünglich aufgegebenen Menge. Einzig Nitrat mit $105 \%$ und Ca mit $47 \%$ fallen aus diesem Bereich heraus. Der Bereich ist als weit einzustufen, und zunächst scheint die Wiederfindung ungenügend zu sein. Wie zuvor beschrieben findet Simkin et al (2004) für Nitrat über 100\%, so dass Ergebnisse grösser 100\% mit der Literatur konform sind. Die hohen Werte werden auf Kontaminationen zurückgeführt, die umso höher ausfallen, je schwieriger die Sicherung der Wasserqualität und der Laborrahmenbedingungen sind. Die Fixierung und die Wiederfindung von Kationen sind in der Literatur selten beschrieben und dem Autor ist nur eine Untersuchung (Crabtree and Trudgill 1981) bekannt, die Werte für die Wiederfindung angibt. Für Ca, $\mathrm{K}$ und $\mathrm{Mg}$ werden Minderbefunde zwischen 2\% und 5\% angegeben. Als Extrakt wurde eine Kochsalzlösung verwendet. Eine Na-Bestimmung war in diesem Versuch nicht möglich. Der Versuch 
wurde mit einer Extraktionszeit von 24 Stunden durchgeführt. Die Messungen in unserer Untersuchung wurden aus Extrakten von 30 min Schüttelzeit mit Schwefelsäure erhalten und ermöglichten eine Na-Bestimmung.

Zur Erklärung sei das Sorptionsprinzip am Tauscher kurz erläutert. Bei der Belegung werden die relativ kleinen und damit mobilen Wasserstoffionen (Protonen) gegen große Moleküle ausgetauscht. Handelt es sich um doppelt oder mehrfach geladene Moleküle, werden diese fester an den Sorbent gebunden. Beim Rücktausch wird mit einer hochkonzentrierten Protonenlösung (Säure) ein Protonendruck aufgebaut, der die gebundenen lonen von den Ladungsplätzen verdrängt. Ein Gleichgewicht zwischen Protonen und auszutauschenden lonen stellt sich ein, das nicht überschritten werden kann.

Aufgrund der Größe und der Ladungsstärke der lonen handelt es sich um Prozesse, die Zeitgebunden sind und sich asymptotisch einem Höchstpunkt mit verlängerten Austauschzeiten annähert. Da diese Prozesse jedoch festen Abläufen (genauen Austauschzeiten, Extraktionsmittelkonzentrationen etc.) unterliegen, wurden aufgrund der hohen Elemententzüge aus den Prüflösungen die geringeren Widerfindungen als ausreichend akzeptiert. Das entzogene, nicht gefundene lonenmaterial aus der Prüflösung muss auf dem Austauscherharz verblieben sein und kann mit einem Faktor hochgerechnet werden. Diese Faktoren werden in der Tab. 2.4 als Korrekturfaktoren für Extraktionen mit kurzen Extraktionsbedingungen angegeben. Trotz einer Vorbehandlung des lonentauschers wurden Blindwerte für Nährstoffe am lonentauscher erfasst. In der vorgelegten Untersuchung wurde an den Messwerten eine Blindwertkorrektur durchgeführt. Die Blindwerte sind in Tabelle 2.5 aufgeführt.

In den vorangegangenen Versuchsanstellungen wurden vor allem statische Untersuchungen mit Monoelement- und Multielementlösungen durchgeführt, um die technischen und physikalischen Grenzen der IER-Kollektoren zu erfassen. Im Folgenden werden Versuche zur dynamischen Eignung der IER-Kollektoren besonders im Bezug auf den Einsatz im Gelände diskutiert.

Niederschlagsereignisse können in den Tropen hohe Niederschlagsintensität erreichen (Gutzler 2011). Dabei können die Intensitäten sowohl innerhalb des Niederschlagsereignisses als auch zwischen den Ereignissen variieren. Als Folge fallen in kurzen Zeiträumen große Wassermengen an, die mit ihrer Nährstofffracht den IER-Kollektor passieren müssen.

Mit der Verminderung der Fließgeschwindigkeit in den geknickten IER-Kollektoren wurden eine Erhöhung der Verweilzeit der Lösung und damit eine Steigerung der Fixierleistung erreicht (Tab. 2.6). Dieser Effekt wird auf eine rein zeitliche Komponente zurückgeführt. Die Steigerung der Fixierleistung betrug im Mittel 3\% über alle Elemente gegenüber einer bereits hohen Fixierleistung in den gestreckten Röhren. Relativ Betrachtet stellt die Passage von 2 I Lösung in Gelände keinen hohen Niederschlagsbetrag da. Hoch ist allerdings die Geschwindigkeit von $8 \mathrm{~mm}$ pro Minute $\left(480 \mathrm{~mm} \mathrm{~h}^{-1}\right)$, die selten, und wenn nur in kurzen intensiven Niederschlagsereignissen erreicht wird. Der gesteckte IER-Kollektor erbrachte ähnliche Fixierleistungen, wie diese in vorrangegangenen Versuchen erfasst wurden.

Dass bei solch hohen Fließgeschwindigkeiten lonen den IER-Kollektor ungehindert durchwandern, ist zu erwarten, da Wasser schnell in weiten Fließkanälen des lonentauschermaterials ohne intensiven 
Kontakt zu den Ladungsplätzen abgeleitet wird. Festzuhalten bleibt, dass es sich hier nicht um einen Durchbruch durch zu hohe Belegung der Austauscherplätze handelt oder Ionen die durch einen Chromatographieprozeß im IER-Kollektor verlagert wurden. Dieses Durchwandern ist mit der Geschwindigkeit der Perkolation gekoppelt und wird auf eine zu geringe Zeit zur Fixierung der Elemente an freien Ladungsplätzen zurück geführt. In weiteren Versuchen wurde das eingefüllte Ionentauschermaterial leicht verdichtet, um eine etwas dichtere Packung zu erreichen.

Aufgrund der bereits hohen gemessenen Leistungen in vorrangegangenen Versuchen und der Tatsache, dass solch hohe Niederschlagsintensitäten selten sind und dennoch gut in gestreckten IERKollektoren erfasst werden, wurden die gestreckten IER-Kollektoren im Freiland eingesetzt. In einem Vorversuch konnten gestreckte IER-Kollektoren erst bei höheren Belastungsmengen zum Überlaufen gebracht werden, während geknickte IER-Kollektoren früher eine Aufnahmegrenze der Niederschlagsmenge erreichten. Durch ein Überlaufen der Trichter würden unbemerkt Depositionsverluste auftreten, die es zu vermeiden galt.

Sehr schlechte Fixierleistungen zeigten die IER-Kugeln, die in Kanistern den Lösungen das Ionenpotenzial entziehen sollten. Fixierleistungen von über $40 \%$ wurden nur für $\mathrm{K}$ erreicht und stellen aufgrund der geringen Lösungsentzüge keine Alternative zu den IER-Kollektoren dar.

Vermutlich ist ein Diffusionsgradient an der Oberfläche der lonentauscherkugel, verursacht durch eine Absättigung der oberen aktiven Schichten im lonentauscher-Beutel (geringe Oberfläche), für die geringen Fixierleistungen verantwortlich. Durch die Belegung der freien Ladungsplätze wird ein Gleichgewicht zu den noch freien Plätzen im Inneren der Kugel sowie zu der Lösung hergestellt. Es tritt eine Diffusionshinderung ein, die in der 24 stündigen Verweilzeit der Kugel nicht überwunden werden konnte. Auf eine weitere Betrachtung und Auswertung des Versuches mit lonentauscherkugel wird daher verzichtet.

Niederschlagsereignisse zeigen während ihres Verlaufes eine Verringerung der Nährstoffkonzentrationen im Niederschlag. Dieser Effekt resultiert daraus, dass in der Luft schwebende Partikel mit einsetzendem Regen ausgewaschen werden. Mit zunehmender Verarmung der Partikel in der Luft sinkt die Nährstoffkonzentration im Regen (Gravenhorst 2000; Fan et al. 2009). Im Verdünnungs- und Durchwaschungsversuch wurde dieses Phänomen berücksichtigt. Dabei zeigte die Variante der immer geringer konzentrierten Lösungen mit einer mittleren um $8 \%$ höheren Fixierleistung einen deutlich besseren Lösungsentzug als die konstant konzentriert aufgegebene Lösung (Tab2.7). Dass geringer konzentrierte Lösungen deutlich besser Fixiert werden, verwundert zunächst. Ursache ist wiederum eine zeitliche Variante, da gleiche Gehalte geringer konzentriert eine längere Perkolationszeit benötigen. Diese „relativ“ langsamere Perkolation bewirkt eine höhere "Ordnung", indem verbleibende freie Plätze am lonentauscher ähnlich dem eines Kristallaufbaus an den freien Plätze nach und nach aufgefüllt werden. Damit wird der lonentauscher dichter belegt. Hinweis auf eine solche Struktur ergeben die leicht höheren Belegungen in den ersten Schichten der IER-Kollektoren mit den verdünnten Lösungen (Abb. 2.4).

Die Untersuchungen, bezogen auf die Anforderungen der IER-Kollektoren an die Gegebenheiten, haben gezeigt, dass die Sammler durch hohen Sammeleigenschaften in Starkregenereignissen gute Fixierleistungen erbringen. Treten sehr hohe Fließgeschwindigkeiten auf, können leichte 
Reduzierungen der Fixierleistungen auftreten. In der Praxis wird dies allerdings eine untergeordnete Rolle spielen, da die höchsten Nährstoffeinträge mit einsetzendem Regen erwartet werden, die selten als Intensivregenfälle mit hohen Intensitäten starten. Werden im Verlauf die Konzentrationen verdünnt, können auch diese gut im IER-Kollektor fixiert werden.

Weitere Vorteile liegen in der Aufkonzentrierung und analytisch vereinfachten Erfassung der Ionen sowie dem vereinfachten Probentransport durch geringe Massen, die keinerlei Kühlung oder Sonderbehandlung erhalten müssen. Eine aufwendige Stabilisierung der Nährstoffe zur Stabilisierung gegen Pilz- und Bakterienabbau ist durch die fehlende Feuchtigkeit und das hohe Protonenangebot aus dem lonentauscher nicht nötig.

Bei beiden Methoden sind jedoch auch Fehler in der Gewinnung und vorbereitenden Präparation der Proben möglich. Bei der Extraktion können Fehler durch die Einwaage des Ionentauschers oder Ungenauigkeit in der Zugabe des Extraktionsmittels oder der Extraktionszeit gemacht werden. Da es sich aber um Laboraufgaben handelt, können diese leicht standardisiert und von geschultem Personal ausgeführt werden.

Bei der Berechnung der Gehalte der Nasssammler aus den Niederschlagswässern ist vor allem die Erfassung der Niederschlagsmengen ein Unsicherheitsfaktor. Kleine Mengen werden nicht oder nur unzureichend erfasst und Verdunstung bleibt oft unberücksichtigt. Bei großen Mengen muss mehrfach ausgelitert werden. Die Wahl zu kleiner Auffanggefäße führt zu Verlusten von Niederschlagslösung und Minderbefunden in der Depositionsmenge. Die Routinen im Gelände sind von täglich bis zweiwöchentlich durchzuführen, während IER-Kollektoren längere Zeiträume je nach Füllmenge integrative Depositionseinträge erfassen können (Fenn et al. 2004). Der Arbeitsaufwand sinkt und birgt damit eine geringere Fehlermöglichkeit. Freiwerdende Kapazitäten können zur Durchführung von Wiederholungen verwendet werden.

\subsection{Schlußfolgerung}

Aus den vorangegangenen Betrachtungen, die von der Literatur bestätigt werden, zeigt sich, dass IER-Kollektoren zur Abschätzung von Depositionseinträgen geeignet sind, wenn auch bezüglich der Blindwerte und Extraktionsleistung einige Einschränkungen gemacht werden müssen. Diese konnten allerdings durch Faktorenbildung ausgeglichen werden. Die Fixierleistung der IER-Kollektoren ist stark abhängig vom Eintragsniveau und verbessert sich mit steigenden Frachten die langsam perkoliert werden.

Mit den Vorteilen im Gelände, einer einfachen Probenahme sowie einer Depositionserfassung ohne Niederschlagserfassung, Transport von stabilen Depositionsproben von geringer Masse können schwierig oder weit entfernte Orte beprobt werden. Aufgrund der stabilen Proben ist sogar ein postalischer Transport denkbar. Durch den passiven Charakter der IER-Kollektoren wird eine bessere Datensicherheit erwartet.

In wie weit eine Unterschätzung oder Überschätzung der Werte stattfindet (Krupa et al. 2000) und für welche Elemente welche Größenordnungen gelten muss, im Feldversuch ermittelt werden. 


\section{Atmospheric ionic deposition in tropical sites of Central Sulawesi determined by ion-exchange resin collectors and bulk water collector}

Der Artikel "Atmospheric ionic deposition in tropical sites of Central Sulawesi determined by ionexchange resin collectors and bulk water collector" wurde im Journal Water Air and Soil Pollution Doi: 10.1007/s11270-012-1211-8 veröffentlicht. 


\section{Atmospheric ionic deposition in tropical sites of Central Sulawesi determined by ion-exchange resin collectors and bulk water collector}

S. Köhler ${ }^{\text {ab }}$, H.F. Jungkunst ${ }^{c}$, C. Gutzler ${ }^{a}$, R. Herrera ${ }^{d}$, G. Gerold $^{a}$

aLandscape Ecology, Institute of Geography, University of Göttingen, Goldschmidtstr. 5, 37077 Göttingen, Germany

bandscape Ecology and Land Evaluation, University of Rostock, Justus-von-Liebig-Weg 6, 18051

Rostock, Germany

'Geoecology / Physical Geography, Institute for Environmental Science; Universitiy of KoblenzLandau, Fortstraße 7, 76829 Landau, Germany

dDepartment of Hydraulic Engineering and Environmental Sciences, Universidad Politécnica de Valencia, Valencia 46022, Spain

E-Mail: stefan.koehler@uni-rostock.de

Tel.:-49381-4983224

Fax: -49381-4983220 


\begin{abstract}
In the light of global change, the necessity to monitor atmospheric depositions that have relevant effects on ecosystems is ever increasing particularly for tropical sites. For this study atmospheric ionic depositions were measured on tropical Central Sulawesi at remote sites with both a conventional bulk water collector system (BWS collector) and a passive ion exchange resin collector system (IER collector). The principle of IER collector to fix all ionic depositions, i.e., anions and cations, has certain advantages referring to (1) post-deposition transformation processes, (2) low ionic concentrations and (3) low rainfall and associated particulate inputs, e.g. dust or sand. The ionic concentrations to be measured for BWS collectors may easily fall below detection limits under low deposition conditions which are common for tropical sites of low land use intensity. Additionally, BWS collections are not as independent from the amount of rain fallen as are IER collections. For this study the significant differences between both collectors found for nearly all measured elements were partly correlated to the rainfall pattern, i.e. for calcium, magnesium, potassium and sodium. However, the significant differences were, in most cases, not highly relevant. More relevant differences between the systems were found for aluminum and nitrate (434-484\%). Almost five times higher values for nitrate clarified the advantage of the IER system particularly for low deposition rate which is one particularity of atmospheric ionic deposition in tropical sites of extensive land use. The monthly resolution of the IER data offers new insights into the temporal distribution of annual ionic depositions. Here it did not follow the tropical rain pattern of a drier season within generally wet conditions.
\end{abstract}

Keywords: Bulk deposition, Central Sulawesi, Passive collector, Nitrate deposition, Phosphorus deposition 


\subsection{Introduction}

Human impact on atmospheric depositions is high (Pye et al. 2009; Fan, et al. 2009; Aas et al. 2007) and particularly depositions of nitrogen $(N)$, sulphur $(S)$, phosphorus $(P)$ and potassium (K) have considerable influence on ecosystems (Cinderby et al. 1998; Adams 2003; Rockström et al. 2009). N and $\mathrm{P}$ cycles are among the most heavily influenced earth system processes by human (Rockström et al. 2009; Phoenix et al. 2006). Even for remote tropical areas, a substantial increase of $\mathrm{N}$ deposition is assumed and its potential impacts are being investigated (Corre et al. 2006; Phoenix et al. 2006). A growing number of studies in the northern hemisphere (Bytnerowicz et al. 2002; Fenn et al. 2004; Fenn and Poth 2004; Gassmann 2004) have shown increasing atmospheric depositions related to human activities and associated environmental impacts (Fan et al. 2009; Aas et al. 2007; Curtis et al. 2009; Phoenix et al. 2006). For example, an excess of $\mathrm{N}$ leads to a multitude of undesirable reactions of the affected ecosystems, which can be: (a) acidification (Adams 2003), (b) contamination of ground and surface water (Curtis et al. 2009) and (c) increasing greenhouse gas emissions, for example $\mathrm{N}_{2} \mathrm{O}$ (Veldkamp et al. 2008). Another major impact of atmospheric $\mathrm{N}$ deposition is the associated loss of richness of species (Phoenix et al. 2006). The latter is extraordinarily important for the tropics which are general biodiversity hot spots. Some studies performed in tropical forests, far from urban areas, have reported alarming increases in deposition rates (Aas et al. 2007; Fan et al. 2009). Thus, monitoring atmospheric ionic deposition across tropical sites is needed and suitable methods have to be explicitly simple and robust to minimise the effects of high temperatures and air humidity. Furthermore, simple economic methods have multiple advantages particularly for poor and remote tropical regions.

In the tropics it is even more difficult than for most other areas to perform precise atmospheric deposition monitoring for a larger area which usually includes very remote sites. Additional to the typically extremely poor accessibility and lack of electricity, which applies for many areas outside the tropics as well, high temperatures and high humidity provoke fast biochemical reactions in the collected samples before they can be analysed. The most relevant problem of "conventional" bulk water collectors (BWS) is concentrations that are too low to be analysed precisely by most common devices. Frequently, samples for nitrate $\left(\mathrm{NO}_{3}-\mathrm{N}\right)$ and $\mathrm{P}$ show concentrations that are below the detection limits for most laboratories, particularly in the tropics. This holds the risk of great uncertainties particularly for low input site that are still near pristine conditions. These sites are especially important as reference sites and monitoring sites to document the expected changes in depositions in the tropics.

A possibility to overcome this general problem of concentrations that are frequently below detection limits is to use passive collectors based on ion exchange resins (IER) which have been tested successfully before particularly for anions (Simkin et al. 2004; Fenn et al. 2002; Fenn and Poth 2004; Templer and Wethers 2011). Our own preliminary tests in the laboratory show general agreement 
with the reported results and confirm the suitability of this method for cations as well. In theory, ions (cations and anions) from rainwater are adsorbed on the surface of the resin during percolation through simple constructed ion exchange resin collectors (IER collectors). Consequently, concentrations rise with time and become more likely detectable when resins are extracted at the laboratory after a longer period. Temporal resolution might be reduced, but on the other hand, it is highly likely that input values would be less uncertain. However, Fenn and Poth (2004) have shown that particularly for the anion $\mathrm{NH}_{4}{ }^{+}$background levels from blank IER collectors can be substantial and have to be considered. Otherwise, artificially high values are monitored. Furthermore, some extraction solutions like sodium citrate do not exchange ions successfully (Simkin et al. 2004). Another limitation of the IER collectors is that the used anion or cation for extraction of course cannot be used for environmental monitoring.

The designs of passive collectors vary and so does the choice of exchange resins of different capacity. Our main goal was to construct an ideal passive collector for the tropics to be used at a monthly resolution scale. The objective was to compare the amount of the total deposition per period collected by the "classic" bulk water sampling method with the values derived from the IER collectors. We expected that by extracting the resin with a small volume of extracting medium, the concentration in the extract would be well above the detection limit. Consequently, our hypothesis was that adapted passive collectors could provide more reliable results for low deposition rates than the "classic bulk water collectors" for aluminum (Al), calcium ( $\mathrm{Ca}$ ), $\mathrm{K}$, iron (Fe), magnesium (Mg), sodium ( $\mathrm{Na}$ ), $\mathrm{P}$ and $\mathrm{NO}_{3}-\mathrm{N}$. A simple, economic design for easy operation in remote field locations would also help in enhancing possibilities to study deposition rates in tropical countries. The objective was to improve our knowledge on atmospheric ionic deposition for Central Sulawesi. The focus was on the particularity of atmospheric ionic deposition in tropical sites with low land use intensity at a monthly resolution. Our goal was to improve the temporal interpretation of bulk depositions. Do bulk ionic depositions mainly follow the rain pattern, i.e. are low depositions typical for the dry season?

\subsection{Materials and Methods}

\subsubsection{Study sites}

The comparisons of the different measuring systems were done at three sites close to the village of Bulili ( $\left.1^{\circ} 12^{\prime} \mathrm{S}, 120^{\circ} 05^{\prime} \mathrm{E}\right)$ near the Lore Lindu National Park, Sulawesi, Indonesia. The sites were located at different altitudes between 632 (site A), 817 (site B) and $926 \mathrm{~m}$ asl. (site C), along a mountain transect. The lower sites were near cacao plantations, while the upper site was located near the rainforest. Total average annual rainfall of the research area is around $2,500 \mathrm{~mm} \mathrm{a}^{-1}$. The mean annual temperature in the study area is $24.5^{\circ} \mathrm{C}$, with a mean relative high humidity of $85 \%$. In a test of evaporation at the study site, evaporation under $0.5 \mathrm{~mm}$ per week was measured (Fig. 3.1)(Gutzler 2011). 


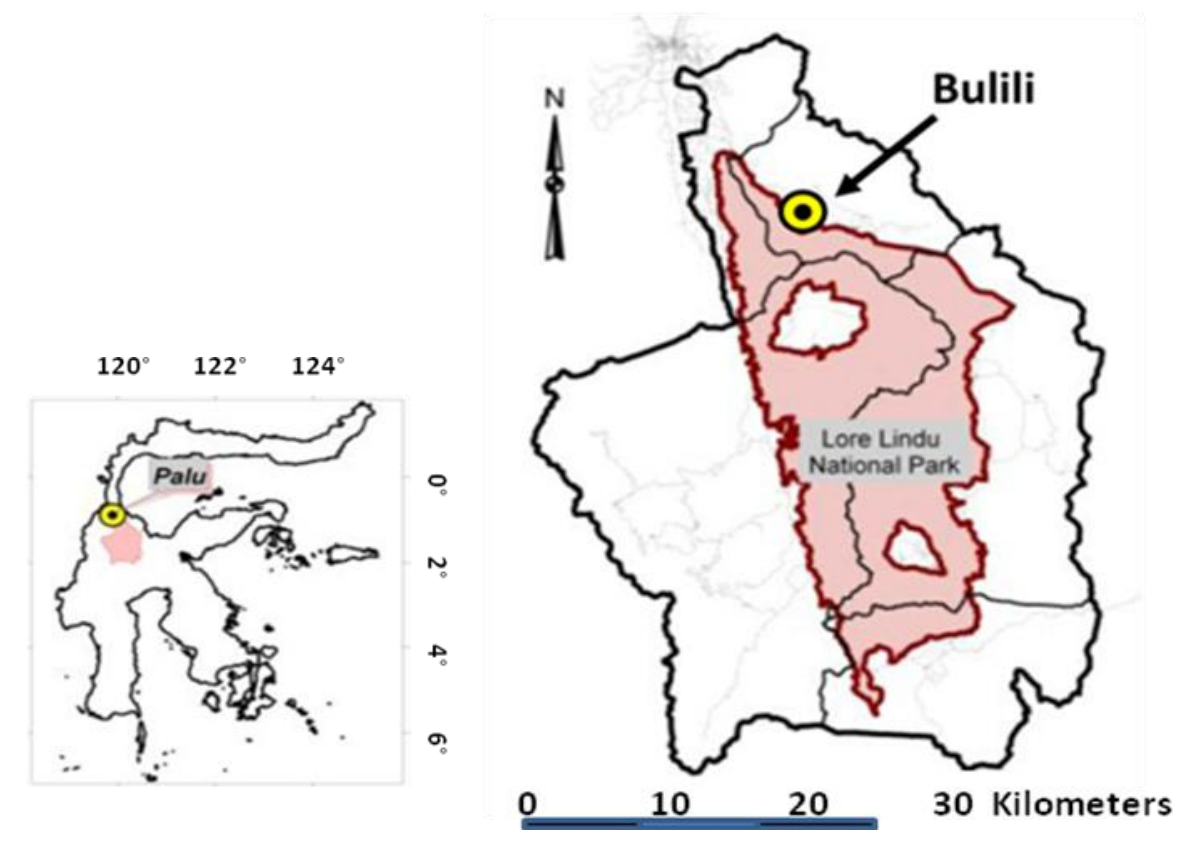

Fig. 3.1: Study site near the Lore Lindu National Park in Central Sulawesi, Indonesia

Abb. 3.1: Untersuchungsgebiet am Rande des Lore Lindu National Parks in Zentral Sulawesi, Indonesien

\subsubsection{Sampler Design}

At each site three pairs of conventional bulk water collectors (BWS collectors) and ion exchange resin collectors (IER collectors) were installed in an area of $2 \mathrm{~m}$ in diameter. Both systems are based on the same collecting principle for bulk deposition from precipitation. The bulk deposition sampler consists of a funnel (polyethylene) of $283.53 \mathrm{~cm}^{2}$ at the top connected to a PVC tube with a diameter of 8 $\mathrm{mm}$. For both systems, the top of the funnel was levelled and fixed to 1 meter above the ground. The construction of the samplers is easy and performed with materials available at local markets.

For the BWS collector, a 75-cm-long PVC tube was inserted into a 5-I can for collecting rainwater. The deposition rate was calculated from the volume and concentration of elements in the rainwater in relation to the funnel area and the sampling interval. Because of tropical high mean temperature, relevant evaporation rates were expected but Gutzler (2011) found no relevant evaporation for the BWS collectors. For the IER collector the same funnel (polyethylene) and tube types were used. The $50-\mathrm{cm}$ tube of the IER collector was closed with a net at the end to retain the resin inside and filled with $50 \mathrm{~g}$ ion exchange resin amberlite MB 20 (Fig. 3.2). Amberlite MB 20 is an anion and cation mixed ion exchange resin with a capacity of about $0.5 \mathrm{mmol}$ charge per gram. The ion content of the rainwater is adsorbed at the surface of the ion exchange resin. These ions can be remobilised later by extracting the ions from the exchange resin at the laboratory using acids or $\mathrm{NaCl}$-solutions. The resin is highly stable even under very low $\mathrm{pH}$ or high salt concentrations. To prevent leaves or insects from entering the column, the resin package was covered with glass wool (Abb. 3.2). For sampling, the IER collector the whole tube filled with ion exchange resin, was replaced at the field site and brought to 
the laboratory for preparation and analyses. IER collectors were tested in the laboratory before the field study.

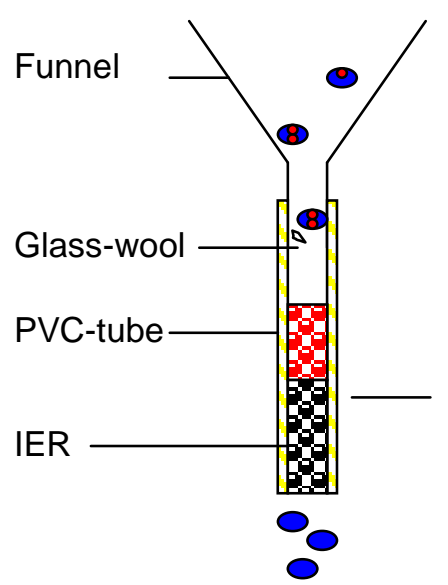

Fig. 3.2: Scheme of an IER-Collector

Abb. 3.2: Aufbau eines IER-Kollektors

\subsubsection{Field Sampling}

The sampling interval for the BWS collector was twice a week. The total amount of rainwater, collected in the 5-I can, was measured with a graduated cylinder. From each BWS collector a 100-m subsample for analyses was taken to the laboratory. To sample the IER collector, the whole tube with the ion exchange resin was brought to the laboratory.

Site B was installed first and sampled twice a month from 26 January 2007 until 30 May 2007. The aim of the higher sampling rate was to test the capacity of ion exchange resin in the field, with deposition rates similar to those given by Nicklas (2006). The following samplings until 31 December 2008 were done once per month. For identifying possible spatial variations of yearly results, additional sites A and C were sampled monthly starting on 29 February 2008 until 31 December 2008.

From the beginning, the elements $\mathrm{Ca}, \mathrm{K}, \mathrm{Mg}$ and $\mathrm{Na}$ were analysed. Starting with the $01 \mathrm{March} 2007$ sampling, $\mathrm{NO}_{3}-\mathrm{N}$, and from 02 August $2007 \mathrm{P}$ and the microelements $\mathrm{Al}, \mathrm{Fe}$ and $\mathrm{Mn}$ were also measured.

\subsubsection{Sample preparation and routine analyses}

The water samples and extract were measured using an inductively coupled plasma-optical emission spectrometry (ICP-OES) 2000 DV (Perkin Elmer). $\mathrm{NO}_{3}-\mathrm{N}$ was analysed using a countinuous flow analysis (CFA) System AA3 Autoanalyser (Bran \& Luebbe). For sample preparation, the water samples had to be filtered with washed $0.45-\mu \mathrm{m}$-filter from Sartorius and directly analysed at the ICP-OES and CFA System. The ion exchange resin subsamples were weighed and homogenised. Approximately a $10-\mathrm{g}$ subsample was taken and extracted twice with $50 \mathrm{ml}$ sulfuric acid (2 M) to determine cations and $\mathrm{P}$. To measure $\mathrm{NO}_{3}-\mathrm{N}$, the extraction of another $10-\mathrm{g}$ ion exchange resin was done with sodium chloride $(1 \mathrm{M})$ solution. In order to correct the calculations of the deposition rate, a blank and an 
extraction factor were measured under the same conditions in the lab with unused ion exchange resin. Therefore, under controlled conditions, unused ion exchange resin was percolated with different concentrated solutions. The percolated water was analysed for unfixed elements passing through to calculate a correction recovery factor for each element separately. For the blank, a subsample of unused ion exchange resin was extracted and analysed. Both factors were used to calculate the results (Tab. 3.1).

Tab. 3.1: Blanks of lon-Exchange Resins and extraction Factor for different elements

Tab. 3.1: Blindwerte des lonentauschermaterials und Extraktionskorrekturfaktoren für Elemente

\begin{tabular}{l|ccccccccc} 
& $\mathrm{Al}$ & $\mathrm{Ca}$ & $\mathrm{Fe}$ & $\mathrm{K}$ & $\mathrm{Mg}$ & $\mathrm{Mn}$ & $\mathrm{Na}$ & $\mathrm{NO}_{3}-\mathrm{N}$ & $\mathrm{P}$ \\
\hline Blank IER $\left(\mathrm{mg}^{*} \mathrm{~g}^{-1}\right)$ & 0.0018 & 0.0039 & 0.0020 & 0.0013 & 0.0051 & 0.0001 & 0.0043 & 0.0009 & 0.0011 \\
Factor & 1.558 & 2.121 & 1.533 & 1.089 & 1.602 & 1.247 & 1.091 & 0.952 & 1
\end{tabular}

\subsection{Results}

The deposition rates determined with both systems for the ions $\mathrm{Ca}, \mathrm{Mg}, \mathrm{K}$ and $\mathrm{Na}$ at site $\mathrm{B}$ exhibited nearly the same cumulative values for 19 months (Tab. 3.2). The relative comparison revealed 3, 7 and $14 \%$ higher values for the IER collectors compared to the BWS collectors for $\mathrm{Mg}, \mathrm{Ca}$ and $\mathrm{Na}$, respectively. For $\mathrm{K}$ the IER collectors sampled $35 \%$ lower values than the BWS collector. $\mathrm{NO}_{3}-\mathrm{N}$ was measured simultaneously by both systems for 18 months and the values for IER collectors showed $434 \%$ higher deposition rates than the BWS collectors. All differences were statistically significant (Tab. 3.2).

Tab. 3.2: The cumulative deposition rates for IER-Collectors and conventional rain collectors at Bulili, field site B, Sulawesi, Indonesia

Tab. 3.2: Kumulative Depositionsraten der IER-Kollektoren und Niederschlagssammlern in Bulili Standort B, Sulawesi, Indonesien

\begin{tabular}{|c|c|c|c|c|c|c|}
\hline & time period month & $\begin{array}{l}\text { BWS-Collector } \\
\mathrm{mg}^{*} \mathrm{~m}^{-1}\end{array}$ & $\begin{array}{l}\text { standard } \\
\text { Devision }\end{array}$ & $\begin{array}{l}\text { IER-Collector } \\
\mathrm{mg}^{*} \mathrm{~m}^{-1}\end{array}$ & $\begin{array}{l}\text { standard } \\
\text { Devision }\end{array}$ & $\begin{array}{c}\text { IER/BWS } \\
\%\end{array}$ \\
\hline \multicolumn{7}{|l|}{ Part A } \\
\hline $\mathrm{Ca}$ & 19 & 1725 & 86 & $1851^{*}$ & 59 & 107 \\
\hline $\mathrm{K}$ & 19 & 1681 & 94 & $1100^{*}$ & 41 & 65 \\
\hline $\mathrm{Mg}$ & 19 & 329 & 16 & $339^{*}$ & 10 & 103 \\
\hline $\mathrm{Na}$ & 19 & 2168 & 118 & $2464^{*}$ & 111 & 114 \\
\hline $\mathrm{NO}_{3}-\mathrm{N}$ & 19 & 49 & 12 & $214^{*}$ & 19 & 434 \\
\hline \multicolumn{7}{|l|}{ Part B } \\
\hline $\mathrm{Al}$ & 12 & 25 & 2 & $121^{\star}$ & 7 & 494 \\
\hline $\mathrm{Fe}$ & 12 & 66 & 4 & 87 & 6 & 132 \\
\hline $\mathrm{Mn}$ & 12 & 16 & 2 & 13 & 1 & 80 \\
\hline$P$ & 12 & 110 & 9 & 127 & 7 & 116 \\
\hline
\end{tabular}

\footnotetext{
* different measuring systems with a significant difference sig $p=0.05$
} 
Furthermore, Fe, Mn, P and Al were measured for 1 year beginning in August 2007 (Tab. 3.2). For P, the difference between both systems was $17 \%$, which is similar to $\mathrm{Ca}, \mathrm{Mg}$ and $\mathrm{Na}$. A comparable difference of $19 \%$ was analysed for $\mathrm{Mn}$, but as for K, the IER collectors collected less than the BWS collector. Slightly higher differences were found for Fe. Again, the IER collectors measured higher deposition of $34 \%$. More relevant and significant differences of $384 \%$ higher inputs were registered with the IER collectors for Al.

At the experimental sites $A$ and $C$, significant differences between the systems were found for $\mathrm{Fe}, \mathrm{Mn}$ and $\mathrm{NO}_{3}-\mathrm{N}$. Annual depositions rates are shown in Tab. 3.3.

Tab. 3.3: Yearly deposition in kg ha-1 a-1 for different elements at three experiment sites

Tab. 3 3: Jahresdeposition in kg ha-1 a-1 für verschiedene Elemente an den drei Versuchsstandorten

\begin{tabular}{c|cccccccc} 
& $\mathrm{Al}$ & $\mathrm{Ca}$ & $\mathrm{K}$ & $\begin{array}{c}\mathrm{Mg} \\
\mathrm{kg} \mathrm{ha}^{-1} \mathrm{a}^{-1}\end{array}$ & $\mathrm{Mn}$ & $\mathrm{Na}$ & $\mathrm{NO}_{3}-\mathrm{N}$ & $\mathrm{P}$ \\
\hline site A & 1.6 & 15.5 & 5.7 & 1.8 & 0.1 & 23.9 & 2.7 & 1.3 \\
site B & 1.1 & 10.4 & 7.5 & 3.2 & 0.1 & 19.6 & 1.4 & 1.2 \\
site C & 1.0 & 14.9 & 10.6 & 2.6 & 0.2 & 20.0 & 2.0 & 1.2
\end{tabular}

\subsubsection{Seasonal variations in precipitation and deposition rates}

In order to study the seasonal variations in deposition rates as related to rainfall pattern, three ions were picked as examples for: (a) lower ( $\mathrm{K})$, (b) higher ( $\mathrm{Ca}$ ) and (c) extremely higher $\left(\mathrm{NO}_{3}-\mathrm{N}\right)$ values measured with the IER collector system as compared to the BWS collector system (Fig. 3.3a-f). During the experimental time, the highest monthly precipitation of $550 \mathrm{~mm}$ was registered for May 2007. November 2007 was the period with the lowest precipitation of $41 \mathrm{~mm}$ during the whole experiment (Fig. 3.3a). The monthly deposition rates of $\mathrm{K}$ measured with the IER collector were rather steady. Only during November 2007 and March 2008 that higher depositions with a wider range were registered (Fig. 3.3b). The deposition rate measured with BWS collectors was, in comparison, more event-driven showing thus high deposition rates corresponding to high precipitation rates. Examples are the months of May till June 2007 as well as March and April 2008 and during the dry periods of November and December 2007 (Figure 3.3c). Similar patterns were found for Ca. Rather steady depositions rates were registered by the IER collectors, whereas somewhat lower deposition rates ( 55 to $87 \mathrm{mg} \mathrm{m}^{-2}$ month $^{-1}$ ) were measured for February until May 2007 (March 2008 was excluded with $153 \mathrm{mg} \mathrm{m}^{-2}$ month $^{-1}$ ) and for February until August 2008 compared to June 2007 until January 2008 which was a period of higher Ca deposition rates (92 to $184 \mathrm{mg} \mathrm{m}^{-2}$ month $^{-1}$ ) (Fig. 3.3d). The Ca deposition rates measured by the BWS collector followed the rain events of May and June 2007 (267 to $290 \mathrm{mg} \mathrm{m}^{-2}$ month $^{-1}$ ) as well as March and April 2008 (111 to $121 \mathrm{mg} \mathrm{m}^{-2}$ month $^{-1}$ ) with higher deposition rates (Fig. 3.3e). 


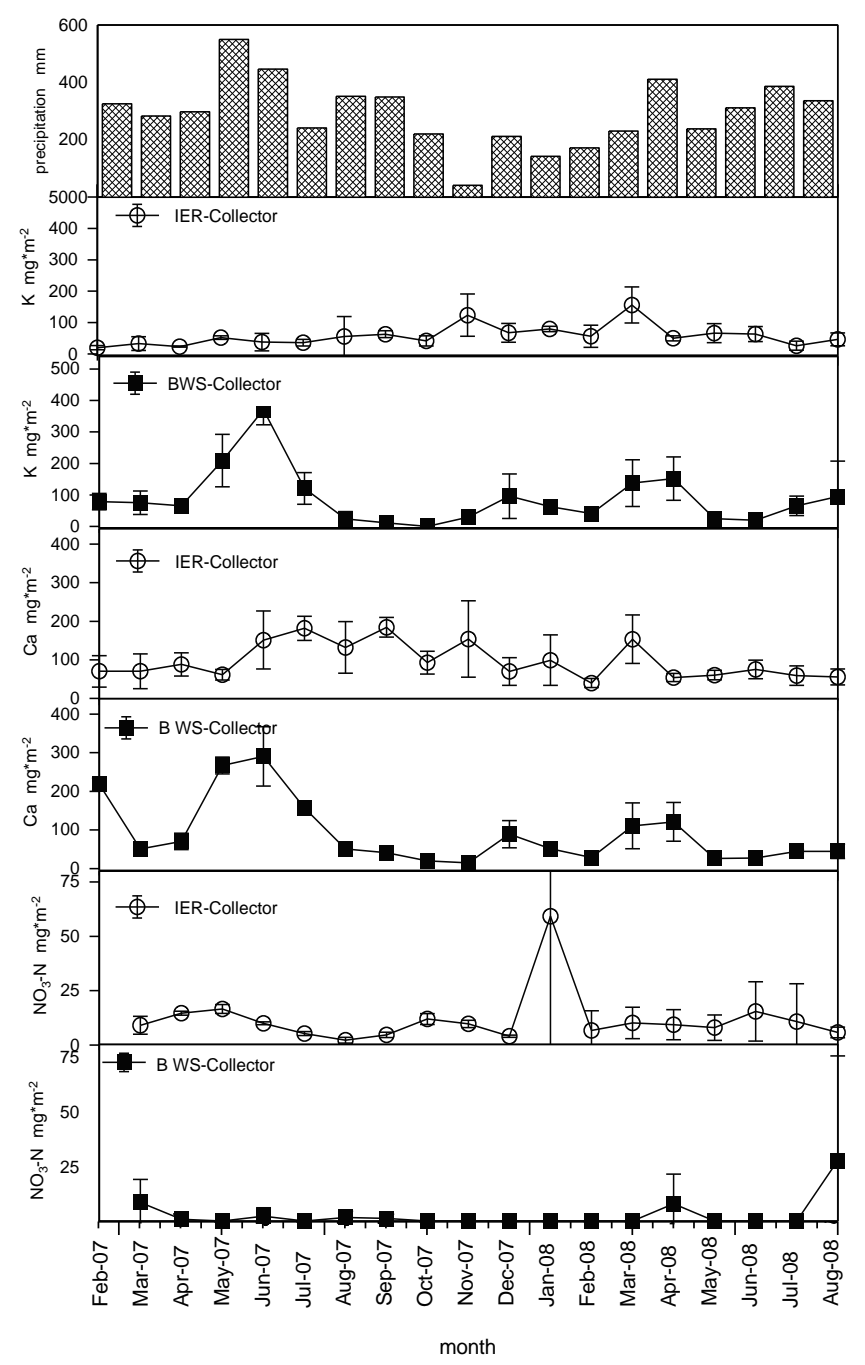

Fig. 3.3 a-e Monthly precipitation is given in a; b-f: deposition rates over the experimental time at the site Open Area for the elements $\mathrm{K}, \mathrm{Ca}$ and $\mathrm{NO}_{3}-\mathrm{N}$ collected with Bulk Water-Collector or lon-Exchange-Resin-Collectors systems.

Abb. 3.3.a-e: Monatliche Niederschlagsmenge (Abb.A) sowie die Depositionsraten während der Untersuchung für die Elemente $\mathrm{K}$, $\mathrm{Ca}$ und $\mathrm{NO}_{3}-\mathrm{N}$, erfasst mit IER-Kollektoren oder Niederschlagssammler

The IER collector clearly revealed more steady deposition rates than the BWS collector particularly for $\mathrm{NO}_{3}-\mathrm{N} . \mathrm{NO}_{3}-\mathrm{N}$ deposition rates were solely registered in 7 out of 18 months with the BWS collector. The high amount in August 2008 was found in one of three replications $\left(0.0,82 \mathrm{mg} \mathrm{m}^{-2}\right)$ for $\mathrm{NO}_{3}-\mathrm{N}$ and for the same sampler, the $\mathrm{K}$ deposition (27,33 and $\left.225 \mathrm{mg} \mathrm{m}^{-2}\right)$ was extremely high. For this event, a contamination, e.g. by birds, cannot be ruled out. Furthermore $\mathrm{NO}_{3}-\mathrm{N}$ depositions were regularly measured only in one of three replications of the BWS collectors. In contrast, the extreme value of $135 \mathrm{mg} \mathrm{m}^{-2}$ found in one IER collector in January 2008 was not related with high $\mathrm{K}$ depositions. The difference between the IER collector and the BWS collector for the monthly deposition of $\mathrm{K}$ and $\mathrm{Ca}$ follows the distribution of precipitation. Higher amounts collected by the BWS collector are usually measured when monthly precipitation was over $220 \mathrm{~mm}$. The same phenomenon was found for the other elements except for $\mathrm{Na}$.

Differences between IER collector and BWS collector for $\mathrm{Ca}$ and $\mathrm{K}$ correlate significantly with precipitation (Fig. 3.4). Exceptions were measured in August and September 2007 (points within the 
ellipse). Since both elements synchronously showed the irregularity, another driver for deposition could be occurring. Without these two points, the relationship between the difference between IER and BWS showed higher significant correlations for $\mathrm{Ca}\left(\mathrm{R}^{2}=-0.81\right)$ and for $\mathrm{K}\left(\mathrm{R}^{2}=-0.75\right)$ with precipitation. No correlation was found for the differences of $\mathrm{NO}_{3}-\mathrm{N}$ and precipitation.

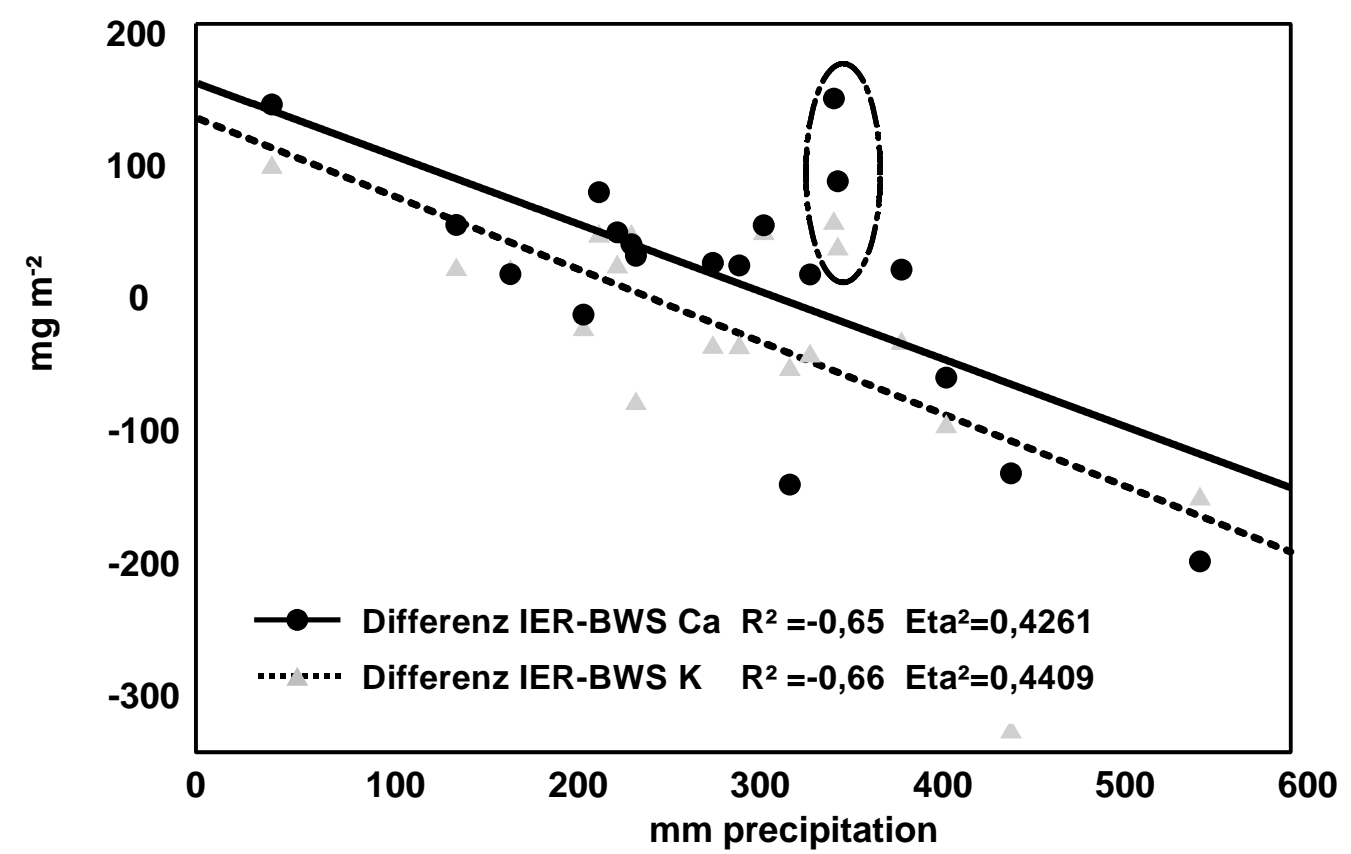

Abb. 3.4: Correlation between the Differences of the deposition rate of Bulk Water-collector and IER-Collector for Ca and $\mathrm{K}$ at the site $\mathrm{B}$

Abb. 3.4: Korrelation zwischen Eintragsdifferenzen der Sammelsysteme IER-Kollekotr und BWS-Sammler für Ca und K am Standort B

\subsubsection{The case of Low deposition}

The concentration of ions, extracted from the samples of the IER collector showed clearly higher concentrations than the water samples from BWS collector (Tab. 3.4). For all elements, $95 \%$ of the IER samples were higher than the lowest standard, $\mathrm{Mn}$ was excluded which showed $83 \%$ from all samples under the $0.1 \mathrm{mg} \mathrm{I}^{-1}$. So, the concentration in most samples from IER collectors falls indisputably within easily detectable ranges of concentrations easily measured in modestly equipped laboratories. For the BWS collector more than $85-97 \%$ of all samples were lower than $0.1 \mathrm{mg} \mathrm{I}^{-1}$. Only for $\mathrm{Na}, \mathrm{Ca}$ and $\mathrm{K}$ most samples were in a range above $0.1 \mathrm{mg} \mathrm{l}^{-1}$ (Tab. 3.4).

Tab. 3.4: Percentage of bulk water- and IER-extract samples below the threshold concentration of 0.1 mg I-1.

Tab. 3.4: Prozentanteile der Proben aus Niederschlagssammlern und IER-Kollektoren die unter dem Grenzwert von 0,1 mg I' ${ }^{1}$ erfast wurden.

\begin{tabular}{l|ccccccccc} 
& $\mathrm{Al}$ & $\mathrm{Ca}$ & $\mathrm{Fe}$ & $\mathrm{K}$ & $\begin{array}{l}\mathrm{Mg} \\
(\%)\end{array}$ & $\mathrm{Mn}$ & $\mathrm{Na}$ & $\mathrm{NO}_{3}-\mathrm{N}$ & $\mathrm{P}$ \\
\hline BWS-Collector & 92.3 & 13.1 & 92.0 & 15.1 & 84.2 & 92.4 & 9.0 & 97.3 & 85.3 \\
IER-Collector & 7.0 & 0.5 & 4.5 & 4.2 & 1.0 & 83.9 & 4.0 & 4.5 & 1.3
\end{tabular}

Bulk water samples (total): $n(\mathrm{Al}, \mathrm{Fe}, \mathrm{Mn}, \mathrm{P})=570, \mathrm{n}(\mathrm{Ca}, \mathrm{K}, \mathrm{Mg}, \mathrm{Na})=680, \mathrm{n}\left(\mathrm{NO}_{3}-\mathrm{N}\right)=582$ IER samples (total) : $n=380, n\left(\mathrm{NO}_{3}-\mathrm{N}\right)=354$ 


\subsection{Discussion}

The results of the BWS collectors, the method applied by most other authors, generally showed rather low deposition rates. Other studies reporting annual bulk depositions for the tropics were predominately in the range of our IER collector results (Tab. 3.3). Mayer et al. (2000); Veneklaas (1990) and Boy et al. (2008a) reported, for Latin America, similar ranges for Al (0.12-5.2 kg ha-1 $\left.\mathrm{a}^{-1}\right)$, Ca (13-76 kg ha- $\left.\mathrm{a}^{-1}\right), \mathrm{Na}\left(11-31 \mathrm{~kg} \mathrm{ha}^{-1} \mathrm{a}^{-1}\right), \mathrm{Mg}\left(2.2-3.2 \mathrm{~kg} \mathrm{ha}^{-1} \mathrm{a}^{-1}\right)$ and $\mathrm{K}\left(6.9-8.3 \mathrm{~kg} \mathrm{ha}^{-1} \mathrm{a}^{-1}\right)$ as we found for Central Sulawesi but with the IER collectors. The deposition rate for $\mathrm{P}$ was a little higher than those reported in other studies $\left(0.48-0.72 \mathrm{~kg} \mathrm{ha}^{-1} \mathrm{a}^{-1}\right)$, but Boy et al. (2008a) found a similar range of 0.64-1.17 kg ha $\mathrm{k}^{-1}$. No comparable results were found for $\mathrm{Mn}$ deposition and our results were three times higher than those reported for the mountain forest Cordillera Real, Ecuador (Boy, et al., 2008a) and ten times higher than those reported for the native agri- and silvicultural ecosystems of the Brazilian Cerrado (Lilienfine and Wilke 2004). The $\mathrm{NO}_{3}-\mathrm{N}$ depositions measured with the IER collectors were in the range of low depositions rates $\left(1.8-3.4 \mathrm{~kg} \mathrm{ha}^{-1} \mathrm{a}^{-1}\right)$ found in natural rainforests of Ecuador, Costa Rica and Sulawesi (Eklund and Pringle 1997; Boy et al. 2008a; Dechert et al. 2005). These rates were low in comparison to other $\mathrm{NO}_{3}-\mathrm{N}$ deposition rates measured in African secondary lowland forest (8.1-45 $\mathrm{kg} \mathrm{ha}^{-1} \mathrm{a}^{-1}$ ) (Lacaux et al. 1992; Muoghalu 2003) or for SE Brazil (6.6-10.7 $\mathrm{kg} \mathrm{ha}^{-1} \mathrm{a}^{-1}$ ) (Mayer et al. 2000).

Both collector systems showed values that were mostly similar for the total measurement period of 19 months. Nevertheless, the rather small differences were significant except for $\mathrm{P}$, Fe and $\mathrm{Mn}$ (Tab. 3.2). These small differences mainly remained at the annual perspective, but the collector system that was showing higher values changed in a few cases. For example, the IER collectors deposition rate was 22\% higher for K measured from August 2007 until July 2008, whereas for the whole period the deposition rate derived from the BWS collector was 35\% higher (Tab. 3.2). Precipitation of Central Sulawesi is characterised by one dryer period per year. During the 19-month measurements, only one dryer period was included. Consequently, the wetter periods were overrepresented which may explain the above-described phenomena of changing differences between the systems. The differences between the two collector systems were most likely related to the different principle of deposition calculations. For the conventional bulk water collector, the concentrations are multiplied by the amount of precipitation. Therefore, deposition rates measured with conventional bulk water collectors were not independent from precipitation rates and wetter periods are unavoidable associated with relative higher deposition rates. This was not the case for IER collectors as virtually all ions are fixed independent of concentration variations of the different precipitation events (Fig. 3.3). When measuring deposition rates with IER collectors it is even unnecessary to know the absolute amount of precipitation. Consequently, the differences found between the two systems can be an artefact of calculation. This is indicated by the correlation analyses between the differences of the collector systems to the precipitation in FigFig. 3.4. 
These uncertainties are high for BWS if concentrations were close to or below detection limits (Table 3.4). A good example for that was given by the low $\mathrm{NO}_{3}-\mathrm{N}$ deposition rates detected by this study for Central Sulawesi (Figure 3.3). This explained the larger differences between the systems from $35 \%$ up to $484 \%$ found for low depositions of $\mathrm{Al}, \mathrm{Fe}, \mathrm{Mn}$ and $\mathrm{NO}_{3}-\mathrm{N}$ which were mainly lower than $1 \mathrm{~kg} \mathrm{ha}^{-1} \mathrm{a}^{-}$ 1 .

For ions with high to medium deposition rates $\left(>1 \mathrm{~kg} \mathrm{ha}^{-1} \mathrm{a}^{-1}\right.$ ), namely $\mathrm{Ca}, \mathrm{Mg}, \mathrm{Na}$ and $\mathrm{P}$, differences between the collector systems were less than $20 \%$ (Tab. 3.2). Basically, the results of Fenn and Poth (2004), Simkin et al. (2004) and Krupa and Legge (2000) that passive collectors tend to collect slightly higher amounts were confirmed for cations as well.

For a monthly resolution, more relevant and significant differences were found. Particularly, significant differences were found during times of high precipitation (e.g. May 2007) for ions like Ca, $\mathrm{K}$ and $\mathrm{Mg}$ (Fig. 3.3) significant differences were found. But also, during low precipitation (e.g. November 2007), for ions like Al and Fe, significant differences were found the monthly resolution. Therefore, different interpretations of temporal dynamics of ionic atmospheric depositions are possible for shorter term analysis depending on the collector system that was applied. It is somewhat striking that deposition values derived from the IER systems hardly show any seasonality as could be expected by precipitation seasonality (dryer and wetter season of Sulawesi). The results of the IER appear more reliable as problems associated with low concentrations were not given.

\subsection{Conclusions}

The advantage of the classic bulk water collector is the possibility of higher time resolutions and the comparison to more measurements from other researchers. The advantages of the IER system are: (a) ions are fixed, therefore low concentration at or below detection limits can be avoided and (b) biochemical transformation processes in the field during measuring intervals are less likely. The IER system is easy to install and requires little attention once in the field. Collecting the samples for analyses takes a very short time. Consequently, it becomes easy to train local people to perform the sampling routine exchanging the IER collectors. Sampling atmospheric deposition data from remote and large areas is possible with IER systems and allows complex overviews of temporal and potentially spatial variations of deposition inputs. Our knowledge on the atmospheric ionic composition of depositions in rural tropical sites mostly would improve if more such sites were measured with the IER methods. That is assuming the values derived with the IER method are generally more precise mainly due to the advantage of capturing low deposition rates typical for remote tropical sites. For nearly pristine tropical sites, it becomes crucially important to be able to measure low inputs to be used as reference to monitor future changes particularly for nitrate. This is more likely achieved with IER than with BWS.

Acknowledgments 
We thank the support and the sponsorship of German Research Foundation (DFG) and the staff at the Storma Laboratory, Palu, Indonesia. 


\section{Regional Atmospheric Nutrient Depositions in Central Sulawesi}

Der Artikel „Regional Atmospheric Nutrient Depositions in Central Sulawesi“ wurde zur Veröffentlichung bei der Zeitschrift Erdkunde eingereicht. 


\section{Regional Atmospheric Nutrient Deposition in Central Sulawesi}

\section{with 8 figures; 3 tables and 1 photo}

S. Köhler ${ }^{\text {ab }}$, H.F. Jungkunst ${ }^{c}$, S. Erasmi ${ }^{\text {d }}$, G. Gerold ${ }^{a}$

aLandscape Ecology, Institute of Geography, University of Göttingen, Goldschmidtstr. 5, 37077 Göttingen, Germany

${ }^{b}$ Landscape Ecology and Land Evaluation, University of Rostock, Justus-von-Liebig-Weg 6, 18051 Rostock, Germany

'Geoecology/Physical Geography, Institute for Environmental Science, University of Koblenz-Landau, Fortstraße 7, 76829 Landau Germany

${ }^{d}$ Cartography, GIS and Remote Sensing, Institute of Geography, University of Göttingen, Goldschmidtstr. 5, 37077 Göttingen, Germany

E-Mail: stefan.koehler@uni-rostock.de

Tel.:+49381-4983224

Fax: +49381-4983220 


\begin{abstract}
:
Deposition rates in remote areas caused by anthropogenic emissions permanently rise in Asian countries and elsewhere. Biomasses burning from slash-and-burn activities, besides fossil fuel burning, cause higher rates of atmospheric deposition in forest- and agricultural sites. An investigation of bulk depositions in Central Sulawesi was done at 13 field sites in an area that covers more than $2310 \mathrm{~km}^{2}$. Bulk depositions were collected with verified ion-exchange passive collectors. Our results show that Central Sulawesi still receives mostly low deposition rates. Especially anthropogenic depositions like nitrate are very low between 0.1 an $0.8 \mathrm{~kg} \mathrm{ha}^{-1} \mathrm{a}^{-1}$ but rise up to $2.4 \mathrm{~kg}$ $\mathrm{ha}^{-1} \mathrm{a}^{-1}$ near slash-and-burn areas. For elements like potassium and calcium similar patterns were found. Undistinguished depositions were found for geogenic elements like iron, manganese and aluminum and partly phosphorus. A principal component analyze allowed to differentiate partly between different sources and different element contributions to the total deposition impact. For different land use systems specific deposition rates were calculated. The investigation revealed that regional sources like slash-and-burn and transregional sources like marine or geogenic dust were driven by different metrological parameters determining local deposition. The emissions of slashand-burn relevantly affect nearby areas, but more distanced forest sites inside the National Park appear to be not yet stressed by these anthropogenic activities.
\end{abstract}

\title{
Zusammenfassung:
}

Stoffeinträge steigen permanent durch anthropogen verursachte Emissionen auch in entfernt gelegenen Landschaften Asiens. Ursache hierfür ist neben der Verbrennung fossiler Energieträger auch die Verbrennung frischer Biomasse durch Brandrodung. Zur Messung von Depositionseinträgen für ein 2310 km² großes Gebiet in Zentral Sulawesi wurden 13 Messstellen zur Depositionserfassung mit Passivsammlern eingerichtet. Es konnte gezeigt werden, dass die Depositionseinträge in Sulawesi meist gering sind und für Nitrat-Stickstoff $\left(\mathrm{NO}_{3}-\mathrm{N}\right)$ zwischen 0,1 und $0,8 \mathrm{~kg} \mathrm{ha}^{-1} \mathrm{a}^{-1}$ liegen. Auf Flächen in der Nähe von Brandrodungsgebieten steigt der Depositionseintrag jedoch auf 2,4 kg $\mathrm{NO}_{3}-\mathrm{N} h \mathrm{a}^{-1} \mathrm{a}^{-1}$. Für Kalzium und Kalium finden sich ähnliche Depositionsmuster, während für Aluminium, Mangan und Eisen andere Depositionsfaktoren eine Rolle spielen. Es konnte gezeigt werden, dass für Zentral-Sulawesi für unterschiedliche Landnutzungssysteme unterschiedliche Elementzusammensetzungen eingetragen werden. Depositionsmaterial kann aus lokalen Feuern (Brandrodung), dem Meer oder Staub aus geogenen Quellen stammen. Für die Landnutzungstypen konnten spezifische Depositionseinträge bestimmt werden. Emissionen aus Brandrodungsaktivitäten beeinflussen die Nährstoffeinträge in einem lokalen Umkreis an der Nationalparkgrenze. Waldflächen im Nationalpark sind von diesen Emissionen bisher noch unbeeinflusst.

Keywords: bulk deposition, Indonesia, rural landscape, air transport, land use system, slash-and-burn 


\subsection{Introduction}

Tropical ecosystems are unique in species richness and commonly adapted to low nutrient availability. Global change with high emissions from industries and traffic will lead to increased atmospheric nutrient deposition, in particular nitrogen $(\mathrm{N})$, which most likely will lead to a shift in species composition and richness (Rockström et al. 2009; Phoenix et al. 2006). High nutrient depositions disturb the complex balance of nutrient cycles in ecosystems and specifically adapted species loose living conditions and certain endemic species will be lost.

Anthropogenic emissions, one source for the depositions, are constantly increasing in Asian countries (Aas 2007). Therefore higher nutrient availability in tropical Asian ecosystems is to be expected. Biomass burning with slash-and-burn in tropical rainforest areas is an important anthropogenic source of sulphur and nitrogen-compounds.

Nutrients like calcium or magnesium are primary deposited by dust from geogenic sources or volcanic activities and are limiting factors for plant growth in poor old forest soils (Cuevas \& Medina 1986). These dusts can also contain iron, manganese and phosphorus, which are besides for plant growth necessary to build enzymes and phytohormons and represent essential nutrition facts. Partly potassium could be deposed by dust and is needed for water transportation in plant systems. Marine aerosols are the main source for sodium and chloride, transported by small salt particels of sea sprey. These aerosols can also contain potassium (Andreae and Merlet, 2001 Formenti et al., 2001).

Increasing biomass burning from shifting cultivation or slash-and-burn activities build anthropogenic emissions in different forms of carbon-, sulphur- and nitrogen oxides and aerosols (Crutzen et al. 2005; Andreae and Merlet 2001; Chang et al. 1987) and therefore are an additional source for nutrient depositions in tropical ecosystems besides natural sources. Nitrogen is responsible for photosynthesis activity of plants and is, beside phosphorus, one of the most important and limiting elements in ecosystems of poor soils in forests and tropical agricultural land use systems. Sulphur, an essential element, is part of enzymes in plants. Beside these natural sources especially matter deposition from fuel combustion or emissions from agricultural farms implement nitrogencomponents, sulphuric or carbon-components to ecosystems (Aas 2007; Dechert et al. 2005; Rollenbeck 2010), but the source of sulphuric parts can also be from volcanic activities (Rollenbeck 2010) and remain in the atmosphere only short times.

Generally it was found that marine influenced tropical rain forests mostly receive higher deposition rates than more continental sites (Schroth et al. 2001; Filoso et al. 1999; Hofhansel et al. 2011; Chuyong et al. 2004)). Especially elements like alkali and alkali earth metals are deposed at lower rates at continental sites (Calil et al. 2010; Oziegbe et al. 2011).

Dechert et al. (2005) and Köhler et al. (2012) showed for Sulawesi low to medium depositions for most mineral elements and especially for nitrate, which can be seen as an anthropogenic tracer deposition. Higher rates near slash-and-burn sides were registered in Central Sulawesi. Sulawesi is an 
island with low industrial production and limited animal production in bigger units. Therefore, the impact of slash-and-burn on atmospheric depositions is clearer detectable than at sites with miscellaneous anthropogenic sources. Large areas of slash-and-burn were observed the border of the Lore Lindu National Park and these are still increasing like results from Erasmi et al. (2010) show. Additional anthropogenic depositions as smoke from forest fires in Kalimantan 2007 (Sundarambal et al. 2010) occasionally are observed in Central Sulawesi.

This study investigates the regional differentiation of atmospheric bulk deposition in relation to land use types and deforestation activities at the border of the Lore Lindu National Park in Central Sulawesi. The main aim was to gain knowledge about the spatial variability of deposition levels for the Lore Lindu national park within an area of about $2310 \mathrm{~km}^{2}$ and the influence of surrounded land use systems on deposition patterns. Due to the isolated state of the island of Sulawesi low depositions especially for anthropogenic matter from industries were expected but with regional diversification including some moderate hot spots originating from land use.

We wanted to point out how natural deposition contributes to the mineral nutrient status in forest ecosystems around the Lore Lindu National Park. We investigated if the influence of local fires is more relevant than regional anthropogenic deposition. Seasonal higher input of local nutrient deposition from slash-and-burn activities were supposed and as a result typical local distribution pattern were expected. Therefore at 13 sites around the National Park, under different land use systems from intensive slash-and-burn activity to nearly natural sites were equipped with passive collectors. Sampling was done in a monthly resolution for aluminium (Al), calcium (Ca), iron (Fe), potassium $(\mathrm{K})$, magnesium $(\mathrm{Mg})$, manganese $(\mathrm{Mn})$, nitrate- $\mathrm{N}\left(\mathrm{NO}_{3}-\mathrm{N}\right)$, sodium $(\mathrm{Na})$ and phosphorus (P).

Our hypothesis was that local increasing slash-and-burn activities influence the surrounding ecosystems in small pattern with anthropogenic depositions of nitrate and phosphorus. Furthermore we expected marine influence with high depositions from long range sources. We also expected a geographical gradient (distance and topography) from the sea to the interior of the island or from anthropogenic activities outside the National Park into the Park.

\subsection{Materials and methods}

\subsubsection{Study sites}

The Lore Lindu National Park is located in the centre of Sulawesi, which is an island of the Indonesian archipel. This National Park $\left(1^{\circ} 18^{\prime} \mathrm{S}, 120^{\circ} 5^{\prime} \mathrm{E}(\right.$ ast $\left.)\right)$ spreads over an area of more than 231000 ha mostly covered by lowland and sub mountain rainforests with altitudes starting at $200 \mathrm{~m}$ asl. up to $2610 \mathrm{~m}$ asl.. The area is affected by the inner tropical convergence zone (ITC). Total rainfall in the research area is approximately $2500 \mathrm{~mm}$ year $^{-1}$ (Gutzler et al. 2010), varies with relief situation between $1500 \mathrm{~mm}$ and over $2500 \mathrm{~mm}$ and shows seasonality in a long term observation of thirty years (Berlage 1949). According to Aldrian and Susantu (2003), the Lore Lindu National Park is located in an intermediate zone of the main Indonesian climate regions with a yearly rainfall regime that is strongly influenced by the Asian-Monsoon-System. Between March and June variable, humid 
south-easterly winds reach eastern Sulawesi, causing the first rainy season of the year. Then the south-easterly winds from the dry and wintery Australian landmass prevail, initiating the dry season from August to October. The second shorter, but even stronger rainy season from November to December is generated by humid north-westerly winds from Kalimantan (Whitten et al. 2002). As a result of the vicinity to the equator (ITC) the area is influenced by convective rainfall during the whole year. The total amount of yearly rainfall is spatially variable and depends largely on topography and elevation. Valleys like the Palu valley, which are situated in a north-south direction, or intramontane basins like Gumbasa valley and Wanga/Watumaeta are in the rain shadow of the steep surrounding mountains.

The main rainy season is from March to June (ITC and SE-monsoon) and a secondary maximum in November (NW-monsoon) (Whitten et al. 2002, Fig. 4.1). At Nopu Open Area (field site, Fig. 4.3) 2007 (3452 mm) and 2008 (3059 mm) yearly rainfall were above the annual average of $2500 \mathrm{~mm}$ but with a dry November $2007(41 \mathrm{~mm})$. In average each month has rainfall amounts of above $100 \mathrm{~mm}$. After Kleinhans (2004) and Gutzler (2011) 50-60\% of rainfall events lies between 7-62 mm with an average of 40 events with more than $12.6 \mathrm{~mm} \mathrm{~h}^{-1}$ intensity (2002-2005, De Vries unpublished).

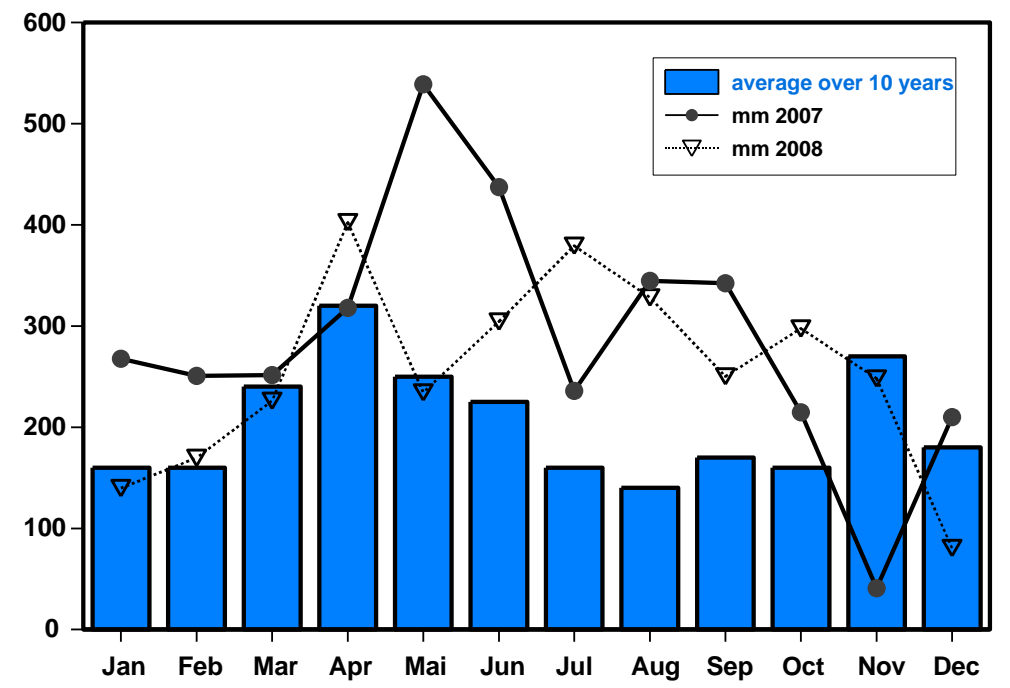

Fig. 4.1: Monthly precipitation during the time of investigation at the study site Nopu Open Area (data: Gutzler (2011) and monthly average from station Lindu and Kaipirore over 10 years (1931-1941) according to the report of Berlage (1949) modified

Abb. 4.1: Monatliche Niederschlagssumme während des Untersuchungszeitraumes am Standort Nopu Open Area (Datenmaterial: Gutzler 2011) und die monatlichen mittleren Niederschläge über ein 10-jähriges Mittel der Standorte Lindu und Kaipirore (1931-1941) nach Berlange (1949) verändert.

Land use systems at the border of Lore Lindu national park are mainly cacao agroforestry systems run by smallholders and annual crops partly in a slash-and-burn manner (Clough et al 2009, Juhrband et al 2010). The deforestation rate at the border and inside the national park is increasing since 2001. The annual rate of deforestation was $0.32 \% \mathrm{a}^{-1}$ (Fig. 4.3) from 2001-2007 according to Erasmi (2007). First annual crops, mainly maize, were planted for 2-3 years after slash-and-burn activities, followed by cacao planting. Burning activity is concentrated during the drier periods, i.e. mostly January and February, before the main rainy season starts. 
The research area with the main fire sites in 2007 and 2008 are shown in figure 4.2. The Modis hotspots show only the bigger fires, because smaller slash-and-burn activities are mostly too small to be detected by the satellites or the weather conditions are not favourable. The main fire activities are concentrated in the area of Napu valley near Wanga and Talabosa. The smaller slash-and-burn fires at Nopu between November 2007 and March 2008 were not visible by Modis but were observed by the storma team during permanent research for the experimental catchment. During this time solely the fire at the 11.01.2008 in Nopu Valley were recognized by Modis.

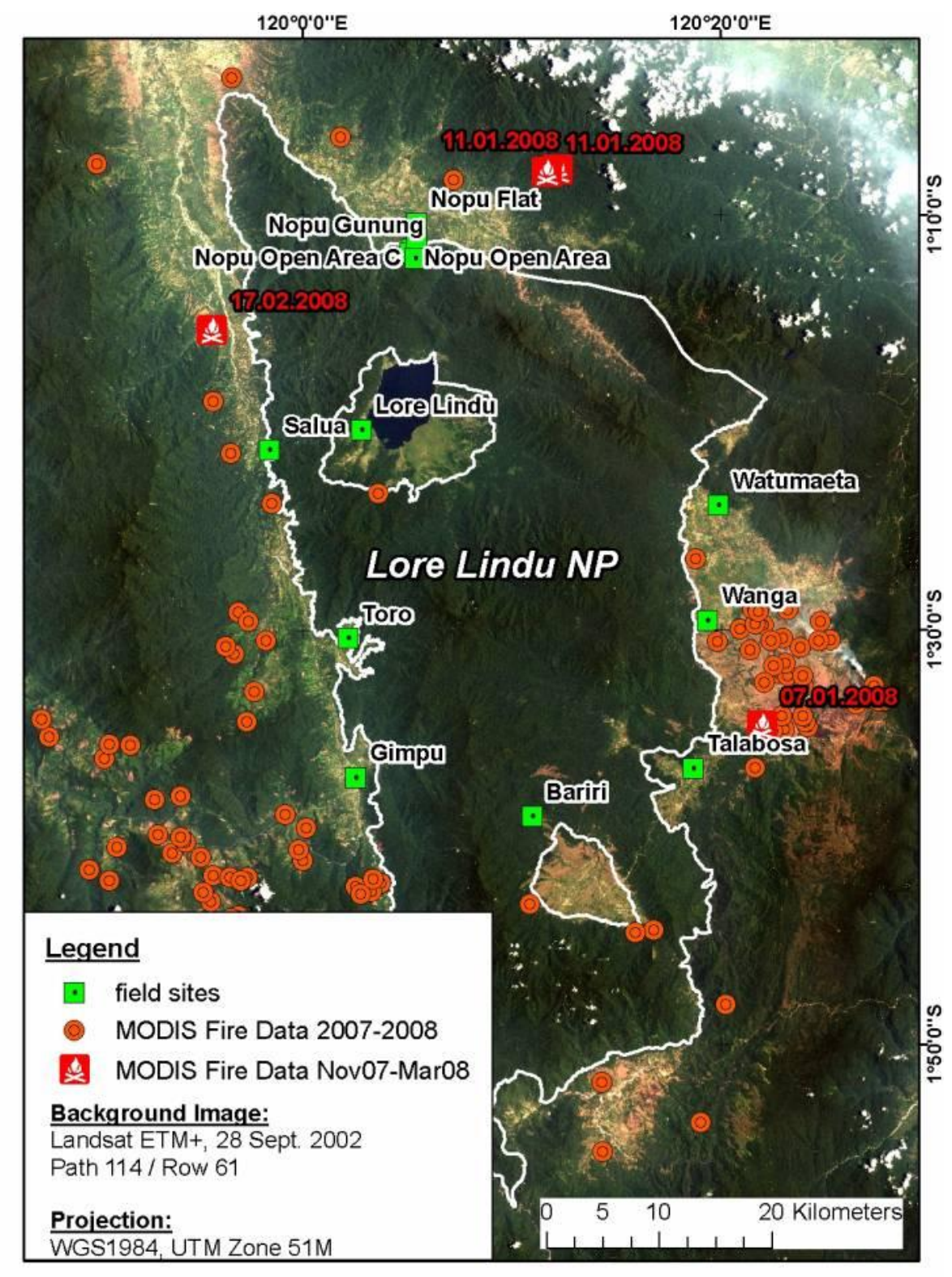

Fig. 4.2: Overview of field sites and fire hotspots in Central Sulawesi, Indonesia for the time period 2007-2008. Data about fire hotspots are taken from the "LANCE FIRMS MODIS Fire/Hotspot" archive at http://earthdata.nasa.gov/firms (last access 08 May 2012). Details about the MODIS Fire product are available from Giglio et al. (2003).

Abb. 4.2: Übersichtskarte des Lore Lindu National Parks mit Brandereignissen im Untersuchungszeitraum (2007 und 2008) in Zenral Sulawesi. Die Daten wurden dem "LANCE FIRMS MODIS Fire/Hotspot" Archive entnommen unter 
http://earthdata.nasa.gov/firms (zugriff: 08 Mai 2012). . Eine weiterführende Beschreibung der Modis Feuerdaten ist unter Giglio et al. (2004) verfügbar (Karte nach S. Erasmi, persönliche Mitteilung).

\subsubsection{Field Sampling}

Atmospheric depositions were monitored at 13 sites around the Lore Lindu National Park area and two sites inside the park (Fig. 4.3). Altitudes range from $364 \mathrm{~m}$ asl. at Salua to $1374 \mathrm{~m}$ asl. in Bariri. Passive collectors in three replications were used to monitor depositions at the singles sites. The sampling started on $2^{\text {nd }}$ of January 2007 and ended $2^{\text {nd }}$ of January 2009 . From the start until $31^{\text {st }}$ of May 2007 the sampling interval was twice a month and afterwards at a monthly basis for all locations except for the Nopu Valley sites (Nopu Flat, Nopu Open Area and Nopu Gunung) which were sampled twice a month until August 2007. At the hillside area to the border of Lore Lindu National Park near the village Nopu, where slash-and-burn activities are registered since 2004/2005, the sites Nopu Open Area and later the sites Nopu Open Area Plot A and Nopu Open Area Plot C were installed at $26^{\text {th }}$ January 2007 and $15^{\text {th }}$ August 2007, respectively. 


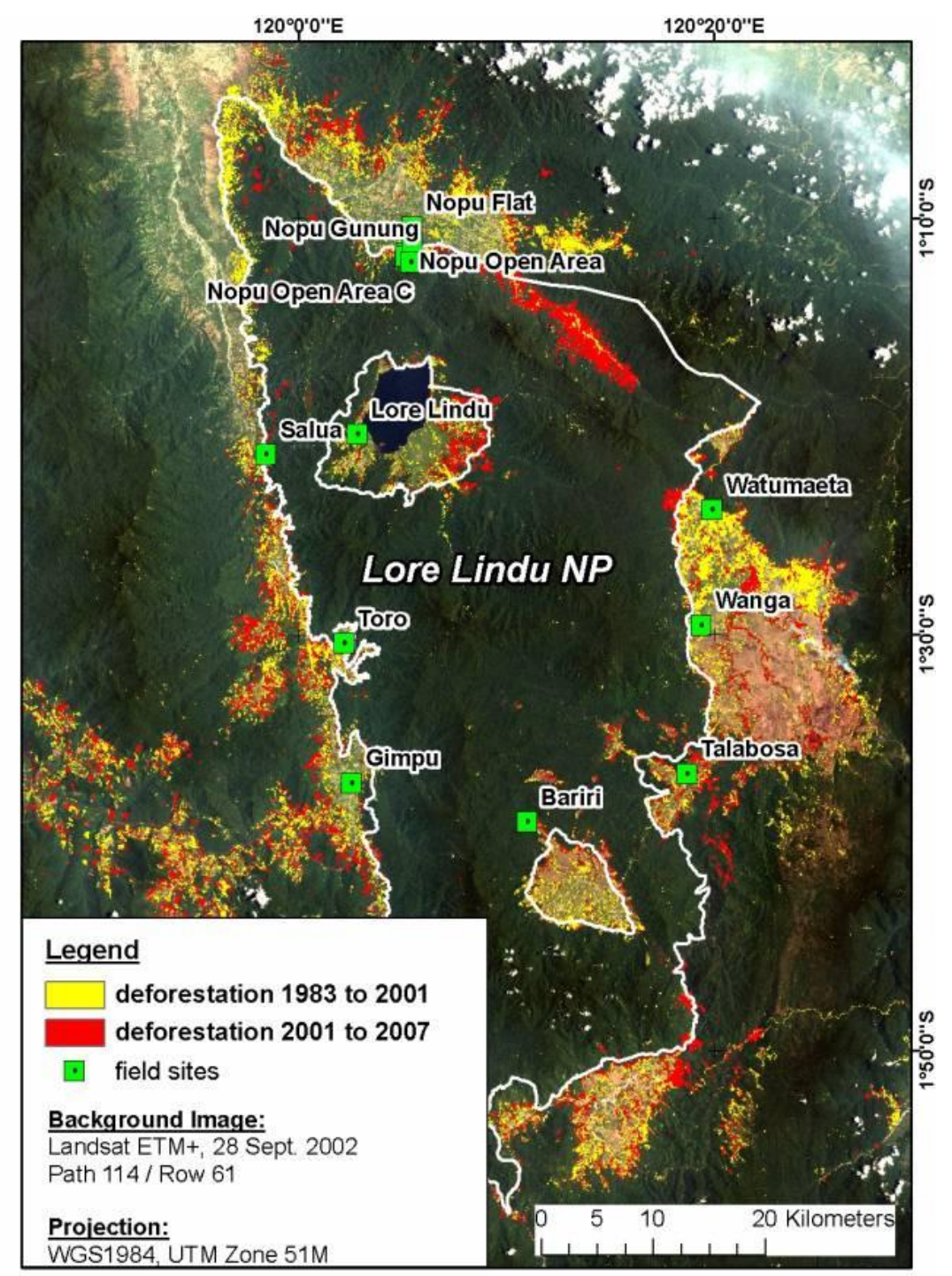

Fig. 4.3: Overview of the study area Lore Lindu National Park with spatial patterns of deforestation activities.

Abb. 4.3: Karte des Lore Lindu Nationalparks mit den Untersuchungsstandorten und den Entwaldungsflächen in unterschiedlichen Zeiträumen

\subsubsection{Sampling Design}

At each site, three Ion Exchange Resin Collectors (IER-collectors) described in Köhler et al. (2012), were installed in an area of $2 \mathrm{~m}$ in diameter (Fig. 4.4). The bulk deposition sampler consists of a funnel of $283.53 \mathrm{~cm}^{2}$ at the top connected to a PVC-tube with a diameter of $8 \mathrm{~mm}$ and filled with ion exchange resins. The whole IER-Samples containing the tube with ion exchange resin were brought to the Storma laboratory in Palu and analysed there. 


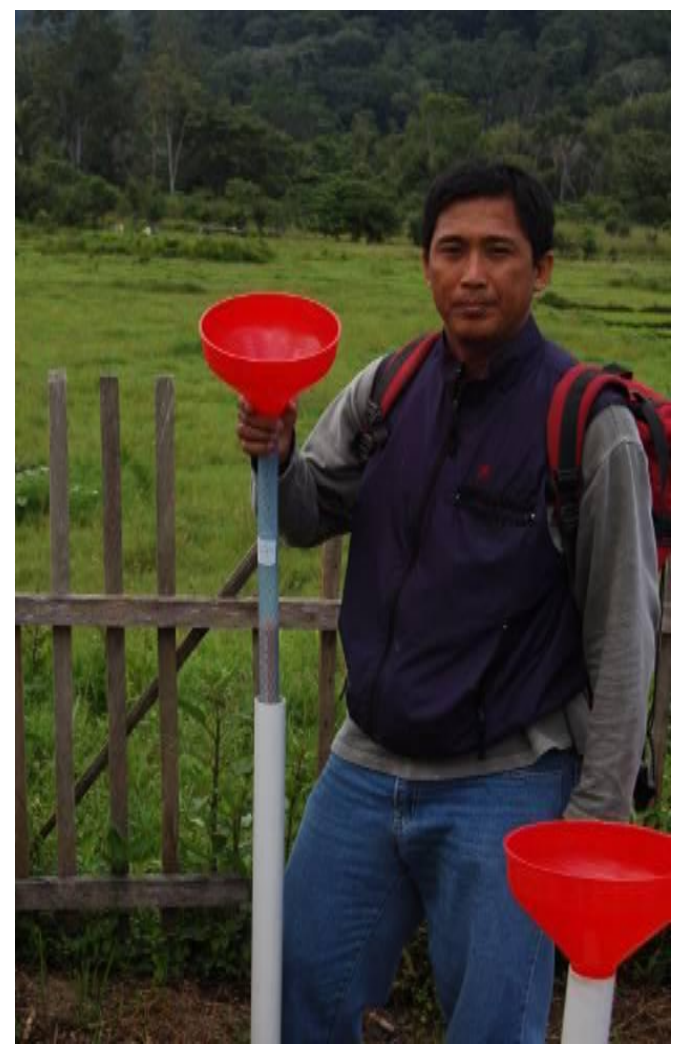

Fig. 4.4:IER-Collector at the field station

Abb. 4.4: IER-Sammler im Feldeinsatz

\subsubsection{Sample preparation and routine analyses}

The ion exchange resin samples were weighed and homogenized. Approximately a $10 \mathrm{~g}$ subsample was taken and extracted twice with $50 \mathrm{ml}$ sulphuric acid $(2 \mathrm{M})$ to determine cations and $\mathrm{P}$. To measure $\mathrm{NO}_{3}-\mathrm{N}$, the extraction of another $10 \mathrm{~g}$ ion exchange resin was done with a solution of sodium chloride (1 M). The extract samples were measured using an ICP-OES 2000 DV (Perkin Elmer). $\mathrm{NO}_{3}-\mathrm{N}$ was analyzed using a CFA-System AA3 Autoanalyser (Bran\&Luebbe). Analysed elements were aluminium (Al), calcium (Ca), potassium (K), iron ( $\mathrm{Fe})$, magnesium $(\mathrm{Mg})$, manganese $(\mathrm{Mn})$ and sodium (Na). Analysed anions were nitrate- $\mathrm{N}\left(\mathrm{NO}_{3}-\mathrm{N}\right)$ and phosphor $(\mathrm{P})$.

For statistical data analysis the programs SPSS 14 and PAST 2.01 (Hammer et al, 2001) were used. To properly classify the sites according to the different land use systems a cluster analysis (Ward's method) was applied and correlations between element depositions data were tested (Pearson). To get information about the composition of the different elements and the source of the deposed material a principle component analysis (PCA-analyze) was calculated.

\subsection{Results}

The bulk deposition at the different study sites around the Lore Lindu National Park for total deposited mass for the elements $\mathrm{Al}, \mathrm{Ca}, \mathrm{Fe}, \mathrm{K}, \mathrm{Mg}, \mathrm{Mn}, \mathrm{Na}, \mathrm{NO}_{3}-\mathrm{N}$ and $\mathrm{P}$ were unevenly distributed in time and space. In 2007, total deposition varied between $23.2 \mathrm{~kg} \mathrm{ha}^{-1} \mathrm{a}^{-1}$ and $45.7 \mathrm{~kg} \mathrm{ha}^{-1} \mathrm{a}^{-1}$ and between $28.4 \mathrm{~kg} \mathrm{ha}^{-1} \mathrm{a}^{-1}$ and $53.2 \mathrm{~kg} \mathrm{ha}^{-1} \mathrm{a}^{-1}$ in 2008 (Tab. 4.1). Most site specific total deposition 
rates were higher in 2008. This surplus was mainly due to $\mathrm{Na}$ and ranged between $16 \%$ and $69 \%$. At the sites Wanga and Watumaeta the annual total depositions were nearly identical between the years. For Bariri and Nopu Flat total depositions were approximately 10\% lower in 2008 than in 2007. Without Na deposition, bulk deposition were higher at three sites in 2008 namely Nopu Gunung (22\%), Nopu Open Area (10\%) and Gimpu (10\%).

For both years Salua, Nopu Flat and Nopu Open Area were among the group of highest annual deposition rates. The group of Toro, Wanga, Watumaeta and Talabosa exhibited lowest annual deposition rates. Bariri showed high depositions in 2007 and low deposition rates in 2008. The absolute maximum of more than $53 \mathrm{~kg} \mathrm{ha}^{-1} \mathrm{a}^{-1}$ of total bulk deposition was measured at Nopu Open Area A and Nopu Open Area C which were solely investigated in 2008. These two sites also had the highest deposition rates for $\mathrm{NO}_{3}-\mathrm{N}$ and situated in the area of high slash-and-burn activities. Also Rates for elements like Ca, K and Na were relatively high. In 2007 the highest deposited element were Ca (Tab. 4.1), a typical geogenic element and showed nearly the same ranking from highest to lowest annual values as the total bulk deposition rates. This relationship basically sustained in 2008, despite the fact that $\mathrm{Na}$ was the element with highest deposition rates. $\mathrm{K}$ as biogenic and marine aerosol is the third most deposited element in both years. The annual $\mathrm{K}$ deposition was between 1.6 and $5.7 \mathrm{~kg} \mathrm{ha}^{-1}$ in 2007 and increased in 2008 from 2.4 to $10.6 \mathrm{~kg} \mathrm{ha}^{-1}$.

Tab. 4.1: The annual element deposition in $\mathrm{kg} \mathrm{ha}^{-1} \mathrm{a}^{-1}$ at the sites at Lore Lindu National Park for the years 2007 and 2008 ; for $2007 \mathrm{NO}_{3}-\mathrm{N}$-Deposition were measured for 10 months

Tab. 4.1: Jährliche Deposition in $\mathrm{kg} \mathrm{ha}^{-1} \mathrm{a}^{-1}$ an den Untersuchungsstandorten im Lore Lindu National Park für die Jahre 2007 und 2008; in 2007 wurden die $\mathrm{NO}_{3}-\mathrm{N}$ nur für 10 Monate erfasst.

\begin{tabular}{|c|c|c|c|c|c|c|c|c|c|c|c|c|c|c|c|c|c|c|c|c|}
\hline & $\mathrm{Al}$ & $\mathrm{Ca}$ & $\mathrm{Fe}$ & $\mathrm{K}$ & $\mathrm{Mg}$ & $\mathrm{Mn}$ & $\mathrm{Na}$ & $\mathrm{NO}_{3}-\mathrm{N}$ & $\mathrm{P}$ & $\begin{array}{c}2007 \\
\text { total }\end{array}$ & $\mathrm{Al}$ & $\mathrm{Ca}$ & $\mathrm{Fe}$ & $\mathrm{K}$ & $\mathrm{Mg}$ & $\mathrm{Mn}$ & $\mathrm{Na}$ & $\mathrm{NO}_{3}-\mathrm{N}$ & $\mathrm{P}$ & $\begin{array}{r}2008 \\
\text { total }\end{array}$ \\
\hline Year & 2007 & & & & & & & & & & 2008 & & & & & & & & & \\
\hline Bariri & 4.1 & 13.7 & 2.5 & 5.6 & 1.3 & 0.2 & 6.8 & 0.4 & 0.9 & 35.4 & 0.8 & 4.6 & 0.5 & 2.4 & 0.9 & 0.1 & 21.1 & 0.2 & 0.7 & 31.2 \\
\hline Gimpu & 1.4 & 8.3 & 1.2 & 3.2 & 1.3 & 0.1 & 6.3 & 0.6 & 0.9 & 23.2 & 1.1 & 8.7 & 0.6 & 4.3 & 1.8 & 0.2 & 20.8 & 0.3 & 1.4 & 39.3 \\
\hline Lore Lindu & 1.6 & 13.5 & 2.4 & 2.8 & 2.4 & 0.3 & 6.8 & 0.7 & 1.1 & 31.6 & 1.4 & 8.1 & 1.3 & 3.9 & 1.9 & 0.2 & 17.9 & 0.5 & 1.4 & 36.7 \\
\hline Nopu Flat & 2.1 & 19.4 & 2.4 & 5.7 & 2.0 & 0.2 & 11.6 & 1.4 & 1.0 & 45.7 & 1.0 & 11.1 & 1.3 & 8.4 & 2.0 & 0.2 & 14.8 & 0.7 & 1.4 & 40.9 \\
\hline Nopu Gunung & 1.7 & 8.1 & 1.4 & 3.9 & 1.6 & 0.1 & 10.2 & 1.3 & 1.4 & 29.6 & 0.8 & 8.8 & 0.5 & 8.6 & 2.0 & 0.1 & 14.3 & 1.6 & 1.4 & 38.1 \\
\hline Nopu Open Area A & - & - & - & - & - & - & - & - & - & - & 1.5 & 15.5 & 0.7 & 5.7 & 1.8 & 0.1 & 23.9 & 2.7 & 1.3 & 53.2 \\
\hline Nopu Open Area C & - & - & - & - & - & - & - & - & - & - & 1.0 & 14.9 & 0.5 & 10.6 & 2.6 & 0.2 & 20.0 & 2.0 & 1.2 & 53.0 \\
\hline Nopu Open Area & 1.2 & 12.6 & 0.8 & 5.6 & 1.8 & 0.1 & 9.4 & 0.9 & 0.8 & 33.1 & 1.1 & 10.4 & 1.0 & 7.5 & 3.2 & 0.1 & 19.6 & 1.4 & 1.2 & 45.6 \\
\hline Salua & 1.6 & 15.3 & 1.7 & 5.4 & 2.7 & 0.1 & 8.0 & 0.7 & 1.2 & 36.7 & 1.7 & 12.0 & 0.7 & 8.5 & 2.6 & 0.2 & 20.1 & 0.4 & 2.0 & 48.0 \\
\hline Talabosa & 1.1 & 9.4 & 1.8 & 3.0 & 1.2 & 0.1 & 7.2 & 0.6 & 0.8 & 25.3 & 2.3 & 5.8 & 1.9 & 3.2 & 2.1 & 0.2 & 14.3 & 0.1 & 1.1 & 31.0 \\
\hline Toro & 1.0 & 8.3 & 1.0 & 1.6 & 1.1 & 0.1 & 10.1 & 0.6 & 0.7 & 24.4 & 1.1 & 5.5 & 0.8 & 3.7 & 1.1 & 0.1 & 14.7 & 0.8 & 0.7 & 28.4 \\
\hline Wanga & 1.5 & 12.9 & 1.7 & 1.8 & 1.2 & 0.1 & 8.6 & 0.6 & 0.6 & 29.1 & 0.8 & 6.0 & 0.7 & 3.1 & 1.4 & 0.1 & 15.1 & 0.4 & 0.8 & 28.5 \\
\hline Watumaeta & 1.7 & 12.2 & 2.0 & 2.1 & 2.1 & 0.2 & 8.6 & 0.7 & 0.6 & 30.3 & 1.5 & 6.8 & 1.5 & 4.2 & 2.1 & 0.2 & 12.6 & 0.2 & 1.4 & 30. \\
\hline
\end{tabular}

The geogenic elements like Al and Fe were mostly higher deposed in 2007 than in 2008. Only the sites Salua, Talabosa and Toro show lower rates for Al in 2007. Also Ca was higher deposed in 2007 without Nopu Gunung and Gimpu. In contrast, the geogenic and low deposed element Mn showed higher deposition rates in 2008 at most sites. 
The highest depositions for $\mathrm{NO}_{3}-\mathrm{N}$ (anthropogenic ion) were measured in 2008 at Nopu Open Area A and Nopu Open Area C, both more than $2 \mathrm{~kg} \mathrm{ha}^{-1} \mathrm{a}^{-1}$. Additionally the sites Nopu Gunung and Nopu Open Area, also near the slash-and-burn area, were higher than $1.3 \mathrm{~kg} \mathrm{ha}^{-1} \mathrm{a}^{-1}$ in 2008 . All other sites ranged in the low deposition rates between 0.1 and $0.8 \mathrm{~kg} \mathrm{ha}^{-1} \mathrm{a}^{-1}$. An almost similar pattern was found in 2007. The deposition rate varied between 0.5 and $0.9 \mathrm{~kg} \mathrm{ha}^{-1} \mathrm{a}^{-1}$ and only for the sites Nopu Gunung and Nopu Flat depositions were higher than $1.3 \mathrm{~kg} \mathrm{ha}^{-1} \mathrm{a}^{-1}$.

The P depositions, with rates between 1.0 and $1.5 \mathrm{~kg} \mathrm{ha}^{-1} \mathrm{a}^{-1}$ in 2008, were a little higher than in 2007 with a range between 0.6 and $1.0 \mathrm{~kg} \mathrm{ha}^{-1} \mathrm{a}^{-1}$.

A cluster analysis of all element depositions in $\mathbf{2 0 0 8}$ with normalized values excluded $\mathrm{Na}$-depositions (Ward's method) (Fig. 4.5) led to the formation of three main groups. Na was excluded as it dominated al depositions by nearly $50 \%$.

One main group with great differences in total element deposition consist of the Nopu sites (Nopu Open Area A, Nopu Open Area C, Nopu Gunung and Nopu Open Area). The second group contains all other sites without Bariri and Talabosa. Due to low element depositions Bariri and Talabosa were grouped into a separated position. The cluster analyses mainly confirmed the observation stated above those different sites can be grouped in deposition levels. The group of the Nopu sites had the highest depositions.

The place of Talabosa lies between natural and agricultural sites (Fig. 4.5) as agriculture is practiced there and the cluster for nitrate alone shows a closer connection to Nopu sites because of different monthly depositions. Talabosa (Fig. 4.6) is discussed separately from Bariri in the following. 


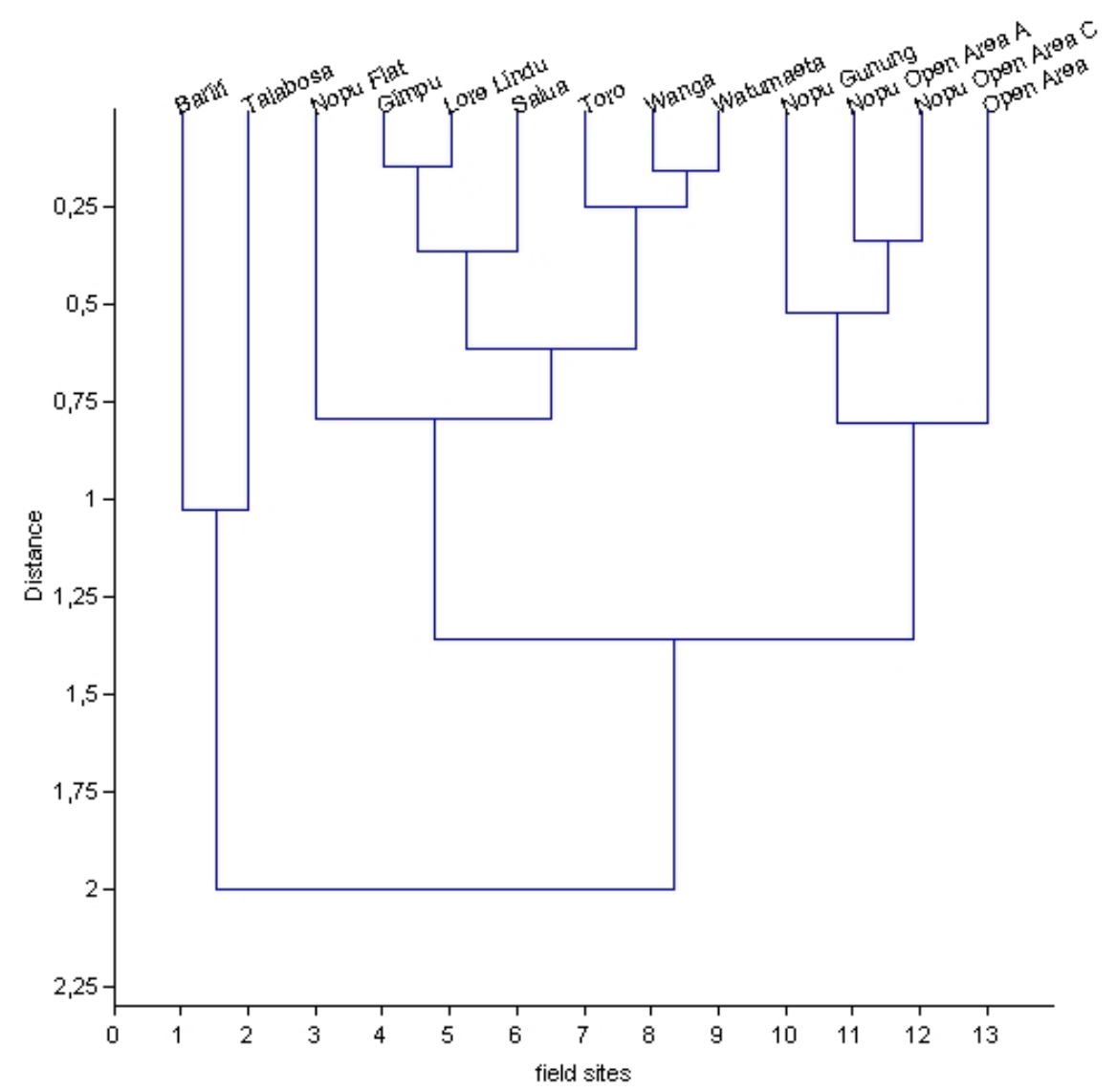

Fig. 4.5: Cluster (wards method) over all elements excluded Na (values zero-one normalized) and all sites for 2008

Abb. 4.4: Cluster für das Jahr 2008 (Wards method) auf der Basis aller Elemente außer Na (mit null-eins normalisierten Werten) für alle Standorte

The strong influence of $\mathrm{NO}_{3}-\mathrm{N}$ originating from slash-and-burn is shown by the cluster analyses done for the $\mathrm{NO}_{3}-\mathrm{N}$ values for 2008 (Fig. 4.6). There all Nopu sites are grouped together, except for Nopu Flat, with a distance of around 500-900 m from Nopu Open Area A and C, which was classified to another group. This elucidates the local effects of slash-and-burn together with the predominant wind direction from slope bottom (Nopu Flat) to upper slopes (Kleinhans 2003). Bariri, with a low deposition of $0.2 \mathrm{~kg} \mathrm{ha}^{-1} \mathrm{a}^{-1} \mathrm{NO}_{3}-\mathrm{N}$, represents in this calculation an own group. Talabosa, with a lower total annual deposition of $0.1 \mathrm{~kg} \mathrm{ha}^{-1} \mathrm{a}^{-1}$, is also separated but near to the other agricultural sites, because the monthly deposition rate is not as homogeneous as in Bariri. In general most sites, without the Nopu sites, show low $\mathrm{NO}_{3}-\mathrm{N}$ depositions under $0.8 \mathrm{~kg} \mathrm{ha}^{-1} \mathrm{a}^{-1}$.

High depositions by $\mathrm{NO}_{3}-\mathrm{N}$ and $\mathrm{K}$ indicate high activities of slash-and-burn at the sites Nopu Open Area A, Nopu Open Area C and Nopu Open Area and are grouped as slash-and-burn sites. The second group contains sites of consolidated agriculture sites (smallholder annual crops and agroforestry (cacao)), like Talabosa, Watumaeta, Wanga, Toro, Nopu Gunung, Salua, Gimpu, Lore Lindu and Nopu Flat. Bariri claims a special position as a remote area near the close rainforest and with low deposition (lowest for $\mathrm{NO}_{3}-\mathrm{N}, \mathrm{P}$ and $\mathrm{K}$ ) and can be interpreted as an own group as natural site. Field observations during these years confirm stable agricultural conditions without new slash-and-burn 
activities at Watumaeta and Talabosa and still natural rainforest conditions at Bariri (see Fig. 4.3, deforestation mainly took place earlier).

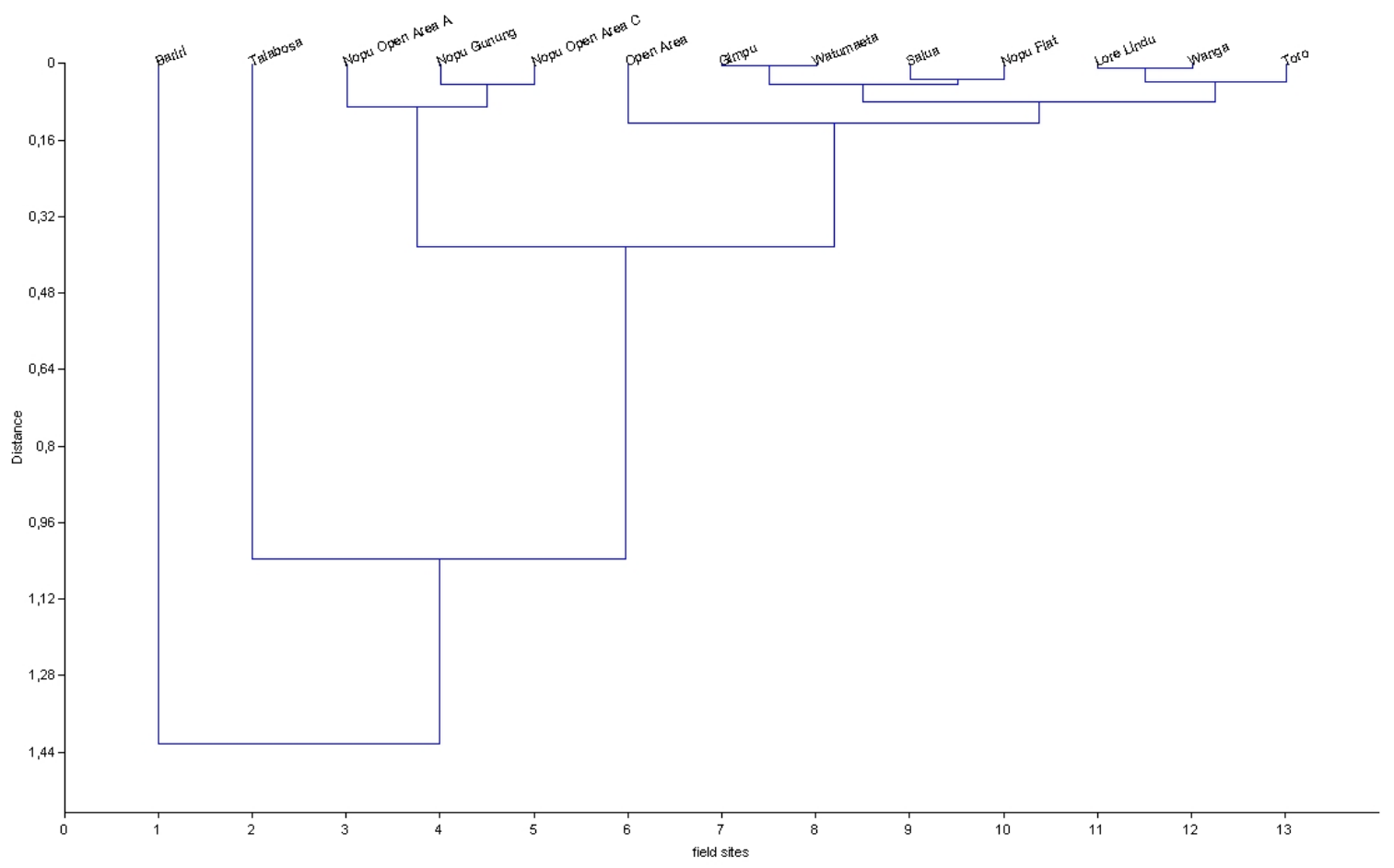

Fig. 4.6: Cluster (wards method, normalized values) based on the $\mathrm{NO}_{3}-\mathrm{N}$ values for 2008

Abb. 4.5: Cluster (wards method) auf der Basis der normalisierten $\mathrm{NO}_{3}-\mathrm{N}$-Daten aus 2008

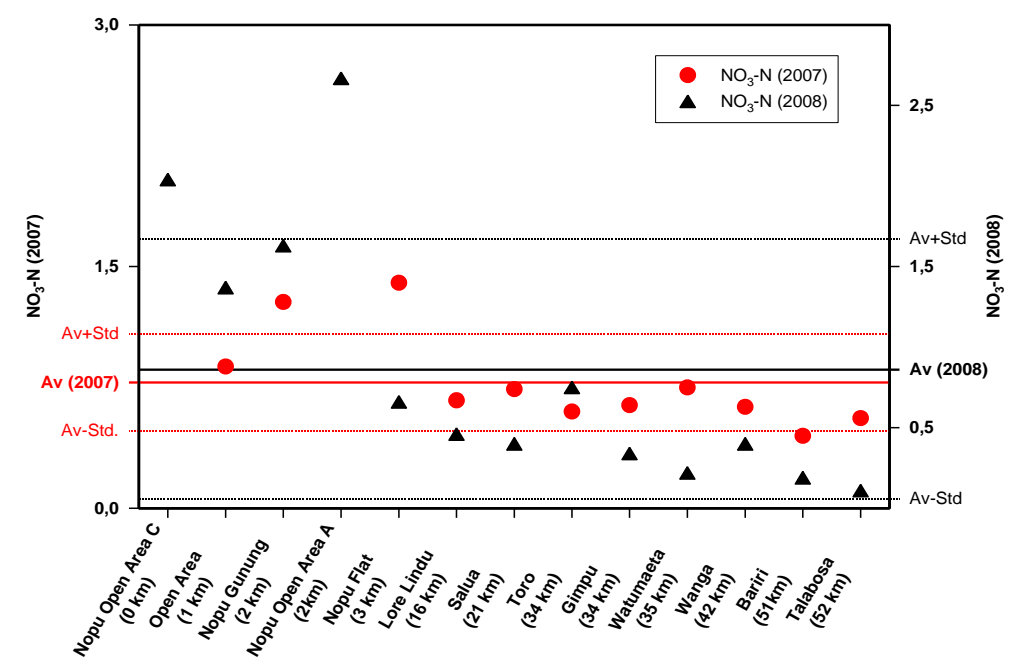

Fig. 4.7: $\mathrm{NO}_{3}-\mathrm{N}$ deposition for 2007 and 2008 in rising distance to the sites Nopu with a high slash-and-burn activity (distance in kilometer $(\mathrm{km})$ from slash and burn area near Nopu Plot $\mathrm{C}$

Abb. 4.6: NO3-N-Deposition an den unterschiedlichen Standorten für die Jahre 2007 und 2008, sortiert nach zunehmendem Abstand in Kilometern ( $\mathrm{km}$ ) von Brandrodungsort nahe dem Standort Nopu Plot A. 
The influence of slash-and-burn for the annual deposition of $\mathrm{NO}_{3}-\mathrm{N}$ is shown in Fig. 4.7. Only the sites Nopu Open Area A, Nopu Open Area $\mathrm{C}$ and Nopu Gunung are above the average (Av) of the $\mathrm{NO}_{3}-\mathrm{N}-$ deposition.

In Tab. 4.2 the average deposition for each land use type for 2008 is shown. The natural site (Bariri) shows for all elements, except for $\mathrm{Na}$, nearly half of element deposition as for other land use systems.

Tab. 4.2: Element deposition average for the different land use types (2008) in $\mathrm{kg} \mathrm{ha}^{-1} \mathrm{a}^{-1}$

Tab. 4.2: Mittlere Elementdeposition für die unterschiedlichen Landnutzungssysteme in $\mathrm{kg} \mathrm{ha}^{-1} \mathrm{a}^{-1}$

\begin{tabular}{l|cccccccccc} 
& $\mathrm{Al}$ & $\mathrm{Ca}$ & $\mathrm{Fe}$ & $\mathrm{K}$ & $\mathrm{Mg}$ & $\mathrm{Mn}$ & $\mathrm{Na}$ & $\mathrm{NO}_{3}-\mathrm{N}$ & $\mathrm{P}$ & Total \\
\hline natural site & 0.79 & 4.59 & 0.48 & 2.38 & 0.88 & 0.10 & 21.13 & 0.19 & 0.68 & 31.21 \\
acgriculture sites & 1.38 & 8.00 & 1.10 & 4.90 & 1.87 & 0.18 & 16.29 & 0.42 & 1.29 & 35.44 \\
slash and burn sites & 1.09 & 12.42 & 0.68 & 8.10 & 2.39 & 0.12 & 19.45 & 1.93 & 1.28 & 47.47
\end{tabular}

The result of a principal component analyse (PCA) show that several sources are responsible for element depositions (Fig. 4.8). For the calculation all element concentrations were normalised.

The second component in the PCA analysis show high loading for $\mathrm{K}$ and high negative loadings for $\mathrm{Al}$ and Fe (geogenic sources). The third component has high positive loads for anthropogenic element $\mathrm{NO}_{3}-\mathrm{N}$ and partly $\mathrm{Ca}$. $\mathrm{P}$ and $\mathrm{Mn}$ has high negative loads.

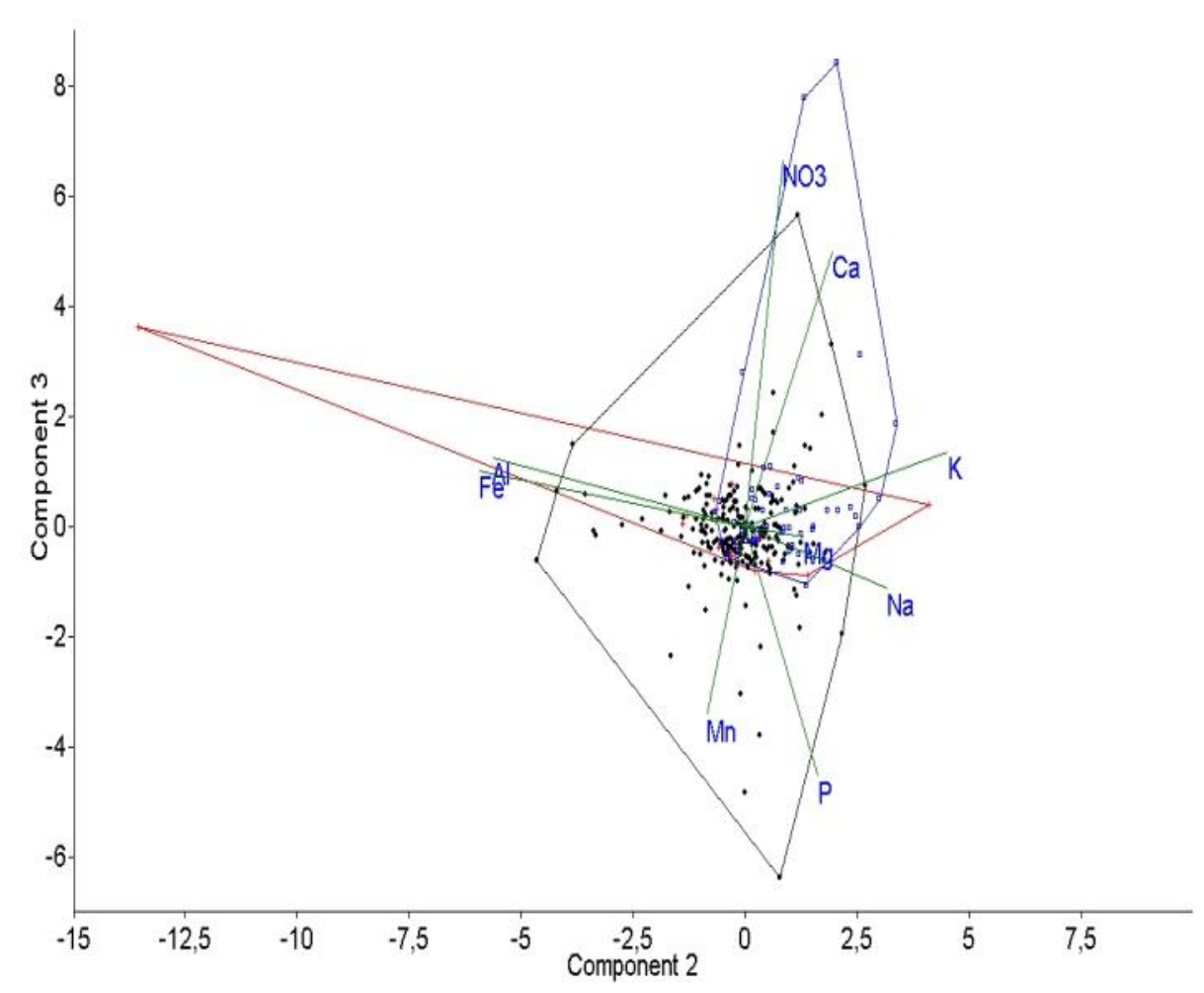

Fig. 4.8: PCA-Analyse for the element deposition at different sites, parted with red colour for natural site (Bariri), blue for slash \& burn (Nopu sites) and black for agricultural sites.

Abb. 4.7: PCA-Analyse der Elementdepositionen aller Standorte sowie einer farblichen Kennzeichnung der Landnutzungssysteme (rot: Regenwaldstandort in Bariri; blau: Brandrodungsflächen in Nopu; schwarz: landwirtschaftliche Nutzflächen) 
$\mathrm{Al}$ and Fe are located near to each other and are trace elements for geogenic dust (Fig. 4.8). A second group is formed by $\mathrm{Na}$ as typical sea salt element (marine) and $\mathrm{Mg}$. Partly $\mathrm{K}$ results also from marine input. $\mathrm{NO}_{3}-\mathrm{N}$ is known as an anthropogenic tracer and lies near to $\mathrm{Ca}$, a typical geogenic element. In the Plot $\mathrm{K}$ is between the elements $\mathrm{Na}$ from sea salt source and $\mathrm{NO}_{3}-\mathrm{N}$ (slash-and-burn source element) and can be interpreted as originating from both sources. For $\mathrm{P}$ highest significant correlation exists with the other elements $\mathrm{Mn}, \mathrm{Mg}$ (2007) and $\mathrm{Mn}$ (2008), which indicates anthropogenic $(\mathrm{P})$ and geogenic $(\mathrm{Mn})$ influence.

The element indicators for slash-and-burn activities $\left(\mathrm{NO}_{3}-\mathrm{N}, \mathrm{K}\right.$ and $\left.\mathrm{P}\right)$ had significant correlations with $r^{2}>0.4$ with $\mathrm{Ca}, \mathrm{Mg}$ and $\mathrm{Mn}$. This may indicate a low bulk deposition level with a mixed signal of marine $(\mathrm{Mg})$ and geogenic/pedogenic $(\mathrm{Ca}, \mathrm{Mn})$ sources.

Therefore marine and geogenic elements showed more or less a spatial homogeneous deposition over the year (Fig. 4.9). For $\mathrm{Ca}$, also a dust deposed element, spatial depositions were not homogeneously distributed around the National Park. At the slash-and-burn site Nopu Open Area a higher depositions corresponded partly with deposition peaks of nitrate and phosphorous.

A deposition peak for $\mathrm{Mn}, \mathrm{Ca}, \mathrm{K}$ and $\mathrm{P}$ exists for June 2007 at the natural site of Bariri. This peak was not confirmed for the other sites and marine $(\mathrm{Na})$ and anthropogenic $\left(\mathrm{NO}_{3}-\mathrm{N}\right)$ deposition remained low.

For $\mathrm{Na}$, the indicator of sea spread deposition, the input increased with the beginning of the main rainy season in November 2007 and reached its maximum in April 2008. The Na-deposition at Bariri and Nopu Open Area A were similar and the deposition in Toro was a little lower, because of the relief position in a small intramontane basin in lee. 


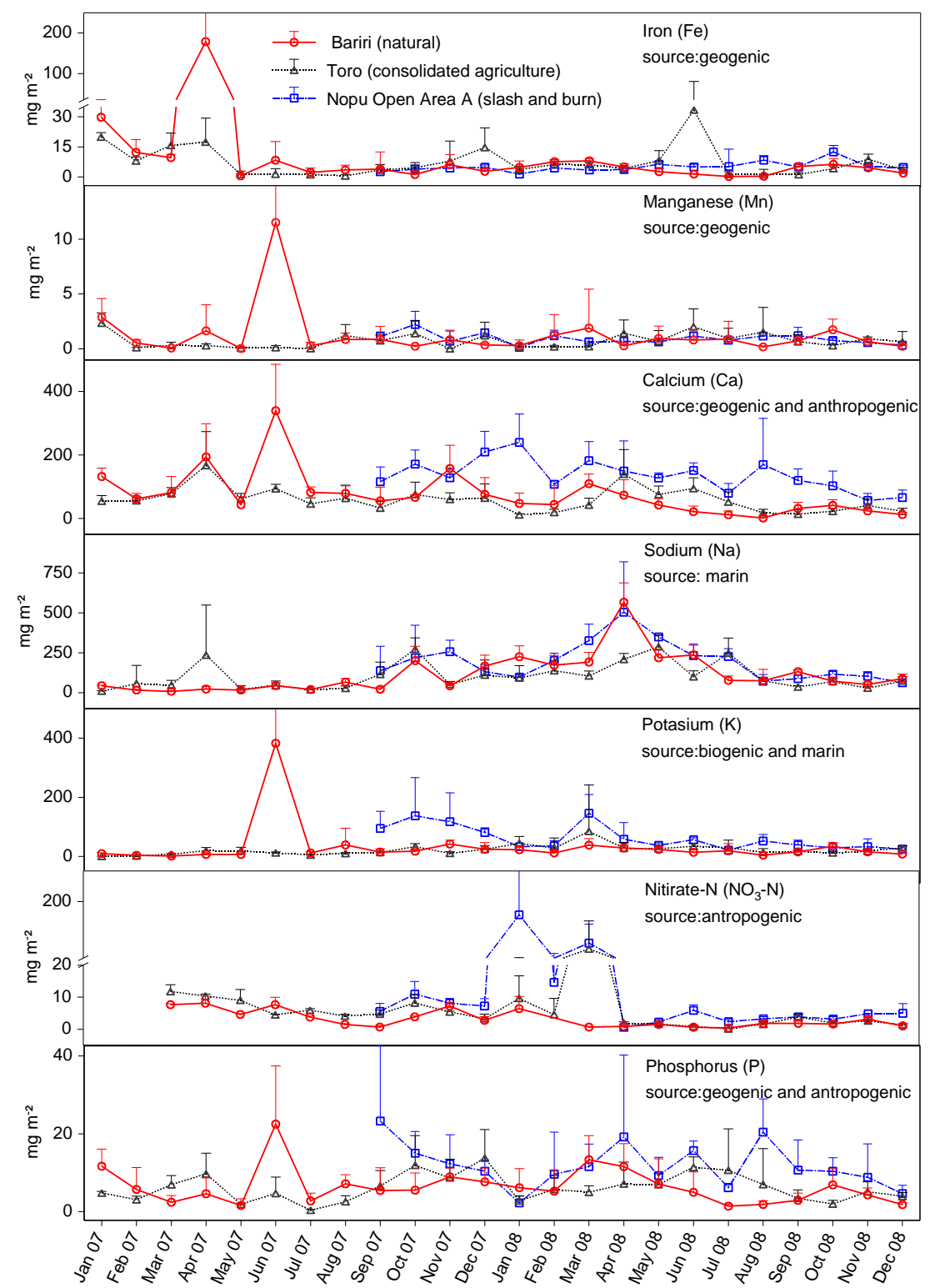

Fig. 4.9: The absolute monthly deposition for different elements at different land use types at Lore Lindu National Park

Abb. 4.8: Monatlicher Depositionseintrag verschiedener Elemente in den unterschiedlichen Landnutzungssystemen des Lore Lindu National Parks

With the start of the dryer season in January 2008 to March 2008 slash-and-burn activity increased and one peak in Toro (March) with $45 \mathrm{mg} \mathrm{m}^{-2}$ and two peaks of $\mathrm{NO}_{3}-\mathrm{N}$ at Nopu Open Area A (January and March) with $157 \mathrm{mg} \mathrm{m}^{-2}$ and $64 \mathrm{mg} \mathrm{m}^{-2}$ were recognized. $\mathrm{NO}_{3}-\mathrm{N}$ clearly reflected the anthropogenic event driven deposition from the local slash-and-burn activities.

The deposition for $\mathrm{K}$ and $\mathrm{P}$ at Nopu Open Area $\mathrm{A}$ showed a similar dynamic like $\mathrm{NO}_{3}-\mathrm{N}$ and was above the other two sites. From May to September 2008 P deposition showed contradicting developments between Bariri and Nopu. This may have been caused by slash-and-burn activities at Nopu. Elevated nutrient depositions of Ca, P and K existed at Nopu since October 2007. 


\subsection{Discussion}

The annual amount of main nutrient input $(\mathrm{Ca}, \mathrm{Mg}, \mathrm{K}$, and $\mathrm{Na}$ ) by wet and dry depositions in the area of Lore Lindu National Park had a range of 28-43 kg ha-1 $\mathrm{a}^{-1}$ (2008). These cation depositions were within a range of 11-37 kg ha $a^{-1} a^{-1}$ given for Lower Montane Rainforests without anthropogenic local or regional impact by Hafkenscheid (2002). Compared to worldwide depositions for tropical rainforests (Tab. 4.3) these amounts seem low. Na-deposition from marine sources was the dominant cation with a proportion of $50-70 \%$, which indicated even less importance of regional element enrichment from anthropogenic sources (e.g. industrial and urban agglomerations). Further the absolute range of $\mathrm{Na}$-deposition (Tab. 4.1 ; $12.6-23.9 \mathrm{~kg} \mathrm{ha}^{-1} \mathrm{a}^{-1}$ ) revealed the general influence of marine air masses with a distance of 50-150 km from measurement sites to the sea. For near marine sites as NP Monteverde in Costa Rica similar Na-depositions occurred (20.5 kg ha ${ }^{-1} \mathrm{a}^{-1}$; Hafkenscheid 2002; 23-33 kg ha-1 a-1; Eklund and Pringle, 1997), whereas continental sites had much lower Nainputs (Rondonia 3,4 kg ha-1 $\mathrm{a}^{-1}$; Filiso et al. 1999; Tab. 3). Our natural site (Tab. 2) showed low $\mathrm{NO}_{3^{-}}$ $\mathrm{N}$ - and $\mathrm{P}$-depositions indicating low anthropogenic influence and showing comparable results to other remote areas in tropical lowland rainforests (Tab. 4.3).

Tab. 4.3: Nutrient fluxes in incident rainfall in tropical lowland rainforests ( $\mathrm{kg} \mathrm{ha}^{-1} \mathrm{a}^{-1}$ )

Tab. 4.3: Stoffeinträge $\left(\mathrm{kg} \mathrm{ha}^{-1} \mathrm{a}^{-1}\right)$ und jährliche Niederschlagsmengen tropischer Tieflandregenwälder

\begin{tabular}{|c|c|c|c|c|c|c|c|c|}
\hline & $\begin{array}{l}\text { altitude } \\
\text { (m a.s.I.) }\end{array}$ & $\begin{array}{c}\text { rainfall } \\
\text { (mm per year) }\end{array}$ & $\mathrm{Ca}$ & $\mathrm{Mg}$ & $\mathrm{K}$ & $\mathrm{Na}$ & $\mathrm{NO}_{3}-\mathrm{N}$ & $\mathrm{PO}_{4}-\mathrm{P}$ \\
\hline \multicolumn{9}{|l|}{ 1-4: continental } \\
\hline Brazil-Manaus (1) & 50 & 2622 & 0.80 & 0.3 & 2.60 & - & 1.40 & 0.07 \\
\hline Brazil-Rio Negro (2) & 90 & 2900 & 2.50 & 0.4 & 0.70 & 2.50 & 0.80 & - \\
\hline Brazil-Rondonia (3) & 143 & 2300 & 17.50 & 1.60 & 8.70 & 3.40 & 0.80 & - \\
\hline Venezuela Gran Sabana (4) & 1300 & 2548 & 0.80 & 1.30 & 2.20 & - & 1.30 & 0.00 \\
\hline \multicolumn{9}{|l|}{ 5-10: marine } \\
\hline Brazil-Rio Grande do Sul (5) & 175 & 1588 & 11.20 & 3.70 & 18.60 & 42.50 & 3.20 & 8.9 (P2O5) \\
\hline Brazil-Mata Atlantica (6) & 10 & 2235 & 5.60 & 1.60 & 6.40 & 75.20 & - & - \\
\hline Costa Rica SW (7) & 70 & 5810 & 20.00 & 5.40 & 6.20 & 6.90 & 2.00 & 0.4 \\
\hline Costa Rica Monteverde (8) & 1500 & 3191 & 5.80 & 2.40 & 3.00 & 20.50 & 1.70 & 0.05 \\
\hline Panama Cord.Central (9) & 1200 & 3510 & 27.90 & 4.10 & 13.50 & 63.50 & - & 0.7 \\
\hline Puerto Rico (9) & 425 & 3750 & 21.80 & 4.90 & 18.20 & 57.20 & - & - \\
\hline Kamerun Korup NP(10) & 150 & 5370 & 9.30 & 5.30 & 7.80 & - & 1.50 & 1.10 \\
\hline Nigeria Ife-Ife (11) & 215 & 1413 & 5.80 & 2.50 & 5.40 & 15.70 & 10.40 & 1.70 \\
\hline Malaysia (9) & 870 & 2700 & 4.00 & 1.20 & 4.00 & - & 5.00 & 0.10 \\
\hline $\begin{array}{l}\text { Sulawesi Lore Lindu National park } \\
\text { (this investigation) }\end{array}$ & 629- 1375 & $\sim 2500$ & $4.6-19-4$ & $0.9-3.2$ & $1.6-10.6$ & $6.8-21.1$ & $0.1-2.7$ & $0.6-2.0$ \\
\hline
\end{tabular}

(1) Schroth et al. 2001; (2) Filoso et al. (1999); (3) Germer et al. (2007); (4) Dezzo and Chacon (2006); (5) Calil et al. (2010); (6) Scheer (2011); (7) Hofhansel et al. (2011); (8) Hafkenscheid (2002); (9) Ashagrie and Zech (2010), (10) Chuyong et al.(2004), (11) Oziegbe et al.(2011) nach Gerold, persönliche Mitteilung 
For geogenic elements like $\mathrm{Fe}, \mathrm{Al}$ or $\mathrm{Mn}$, for all sites less spatially and temporally variable depositions were observed. For these elements slash-and-burn had minor influence as dust production from consolidated agricultural areas (Tab. 4.2). PCA-analysis with same factor loading for Fe and Al show, that they might be useful as a regional "noise" signal of the natural background dust depositions. Most homogenous deposition in space and time was registered for $\mathrm{Mn}$ at rates of 0.1-0.2 $\mathrm{kg} \mathrm{ha}^{-1} \mathrm{a}^{-1}$. $\mathrm{Ca}$, also a geogenic element (Boy et al. 2008; Rollenbeck 2010), revealed larger variability of the deposed mass at the different sites (Tab. 4.1). In particular in 2008, Nopu and Salua had high deposition rates between $10-15 \mathrm{~kg} \mathrm{ha}^{-1} \mathrm{a}^{-1}$ compared with the other sites with a range of 4-8 $\mathrm{kg} \mathrm{ha}^{-1}$ $\mathrm{a}^{-1}$. For lower montane rainforests Hafkenscheid (2002) gave a range between 3.6-5.8 $\mathrm{kg} \mathrm{ha}^{-1} \mathrm{a}^{-1}$, which corresponds well with our average for natural sites (Tab. 4.2). Also Mg lies in the range of natural rainforests (0.5-5.2 kg ha-1 $\mathrm{a}^{-1}$ (Boy et al. 2008; Da Rocha et al 2005, Hafkenscheid 2002). Both elements showed a significant increase from natural to slash-and-burn sites (Tab. 4.2), as indicated by Salua and Nopu in 2008. Mg had different sources that mainly derived from marine and soil components and Ca-deposition originates from dust background and local slash-and-burn activities. In most deposition studies $\mathrm{Ca}$ and $\mathrm{Mg}$ showed significant correlations as it did in our analysis $(r=0.6$, $\mathrm{p}=0.01$ ). But PCA-analysis indicated different origines while $\mathrm{Mg}$ was on the same axe direction as $\mathrm{Na}$ (marine source) and $\mathrm{Ca}$ on the same as $\mathrm{NO}_{3}-\mathrm{N}$ (anthropogenic, slash-and-burn). Both elements were nearly three-fold (three times?) higher in 2008 with similar average yearly deposition at slash-andburn sites as at natural sites. This has to be interpreted that marine sources for $\mathrm{Mg}$ was of minor importance so that dust deposition must have been the main source. This was also elucidated by strong correlations between $\mathrm{Mg}$ and $\mathrm{Al}$, Fe and $\mathrm{Mn}$ (Tab. 1). In general, the depositions of geogenic elements in Central Sulawesi were comparable to other rainforest sites with low input. For the deposition of $\mathrm{Na}$ marine small salt particles are the major source as had been proven at marine rainforest sites (Tab. 4.3).

Seasonal differences for month with similar high rainfall (see Fig. 1, April and July 2008) refer to differences of the origin of the monsunal air masses. Coming from October to April from South China Sea they crossed on the way a long open sea water area were salt particles may be loaded up. During this time the $\mathrm{Na}$-deposition at our field sites permanently rose and maximum deposition rate were reached in April 2008 with $566 \mathrm{mg} \mathrm{m}^{-2}$. This corresponded well with the precipitation maximum (Fig. 4.9; Fig. 4.1). From August to October air masses from the Australian continental site reach the region with lower marine influence. In 2007, higher rain fall was recognised in May and June (Fig. 4.1) with air mass from the Australian continent (Gunawan 2006, Whitten et al 2002). Regional differences between Salua/Lore Lindu/Gimpu/Bariri located at the Palu-Koro-Falt (approximately $\left.20 \mathrm{~kg} \mathrm{ha}^{-1} \mathrm{a}^{-1}\right)$ and Toro/Talabosa/Wanga/Watumaeta (14 $\left.\mathrm{kg} \mathrm{ha}^{-1} \mathrm{a}^{-1}\right)$ also existed in 2008 which revealed the influence of relief position (Lee) of these intramontane basins. Gunawan (2006) reported that land sea wind regime from Palu is detectable at the metrological station in Gimpu.

K-depositions, mainly from biogenic and marine sources, were at a low level, comparable with other natural rainforest sites worldwide (Tab. 4.3). Threefold higher K-input at slash-and-burn sites indicated the anthropogenic impact, mainly at Nopu sites. Even clearer anthropogenic deposition 
signals were given by higher differences between natural sites and slash-and-burn sites for $\mathrm{NO}_{3}-\mathrm{N}$ (ten times higher, Tab. 4.2). Without slash-and-burn $\mathrm{NO}_{3}-\mathrm{N}$ deposition around the Lore Lindu National park were low for most sites $\left(0.2\right.$ and $\left.0.8 \mathrm{~kg} \mathrm{ha}^{-1} \mathrm{a}^{-1}\right)$. Natural remote rainforest sites at Bukit Koto Tabang in Sumatra (Indonesia, $0.6 \mathrm{~kg} \mathrm{ha}^{-1} \mathrm{a}^{-1}$, Ayers, 2002) and in Ecuadorian rainforest (0.3-1.2 $\mathrm{kg} \mathrm{ha} \mathrm{a}^{-1}$, Rollenbeck 2010) showed comparable low $\mathrm{NO}_{3}-\mathrm{N}$ depositions. Higher depositions of 1.4 until $2.7 \mathrm{~kg} \mathrm{ha}^{-1} \mathrm{a}^{-1}$ were found in the area with high slash-and-burn activities in 2008 at Nopu and were consequently grouped together in the cluster analyse in Fig. 4.3. Highest deposition rates corresponded with the dry season (January - March 2008, Fig. 4.1) and main slash-and-burn activities. Higher inputs affect near surrounded areas and most likely changed the natural balance of nitrogen cycles. Comparing our results with the results from Dechert et al. (2008), who reported for two sites near Wanga and Watumaeta $2.6 \mathrm{~kg} \mathrm{ha}^{-1} \mathrm{a}^{-1} \mathrm{NO}_{3}-\mathrm{N}$ in 2002 during a time with high slash-andburn activity, high $\mathrm{NO}_{3}-\mathrm{N}$ deposition may be a temporary phenomena as 5 to 6 years later ranges dropped again to 0.2 to $0.7 \mathrm{~kg} \mathrm{ha}^{-1} \mathrm{a}^{-1}$ (Fig. 4.9). In consequence this means that the anthropogenic deposition in Central Sulawesi is low and the main anthropogenic deposition are from slash-and-burn activities which influence the local surrounding

Rollenbeck (2010) described $\mathrm{PO}_{4}$-sources mainly in association with natural sources, but Mahowald et al (2005) refer to high phosphate levels in precipitation with biomass burning. Levels of P-deposition between 0.2 and $0.7 \mathrm{~kg} \mathrm{ha}^{-1} \mathrm{a}^{-1}$ (Williams et al. 1997; Veneklaas 1990; Muoghalu 2003) were low. Only three sites were in the middle range for 2008. More than two times higher as described for natural sites were found only for Salua in 2008 and could be a result of the fire detected by Modis (Fig. 4.2). Slash-and-burn sites and agricultural sites have no significant difference, which support the assumption that more distanced sources are responsible for P-deposition. Also the local burn of post harvest material, like rice straw could be a possible source. Same axe direction in PCA-analysis for P and Mn (Fig. 4.8) indicated regional diffuse geogenic sources of deposition.

In our investigation the local anthropogenic deposition by slash-and-burn and salt from sea were the main factor for regional diversification in mineral compositions. The low level of element inputs at most sites and throughout the year show low influence of urbanisation and industrial areas in an agricultural dominated landscape with traditional smallholders and still approximately $60 \%$ of submontane and montane rainforest areas. Research on plot based vertical nutrient in- and output at rainforest and cacao plantation sites at Nopu (Gutzler 2011, Gerold 2010) showed, that higher nutrient input $(\mathrm{Ca}, \mathrm{K}, \mathrm{N})$ with slash-and-burn into the soil increased nutrient drainage losses. Furthermore registered higher soil water nutrient contents in the upper rooted soil layer was a temporary effect having the main nutrient peaks around 6-18 months after slash-and-burn activity. The consequences by continued slash-and-burn actives are a higher nutrient input in undisturbed forest sites and higher nutrient losses at agricultural sites.

\subsection{Conclusion}

Loss of tropical rainforests with increasing land use activity and urbanization worldwide contributed during the last 20 to 30 years to increasing mineral element deposition by rainfall also in "natural tropical ecosystems". Therefore site measurements of nutrient input (bulk deposition) in remote 
rainforest areas as for Central Sulawesi has to consider influencing factors of inner tropical circulation, regional effects by topography and land-seawind regime and local effects by slash-andburn activities. A measurement net of 13 bulk deposition sites in Central Sulawesi at the border of Lore Lindu National Park point out: (1) most sites have still a low atmospheric nutrient input, dominated by marine depositions, (2) regional dust background delivers $\mathrm{Al}, \mathrm{Fe}, \mathrm{Mn}$ and $\mathrm{P}$ input and (3) local slash-and-burn activities increased significantly main nutrient input of $\mathrm{Ca}, \mathrm{Mg}, \mathrm{K}$ and $\mathrm{NO}_{3}-\mathrm{N}$. These higher depositions can alter nutrient cycle with changes in the N/P ratios and is therefore subject of ongoing studies on tropical rainforests, including question of plant species and biodiversity shift. For nutrient poor tropical soils with annual cropping systems higher nutrient input may have positive effects, but higher leaching losses can counteract this. Further studies with longer measurement periods in recently dynamic forest conversion regions as Central Sulawesi are necessary and can contribute to answer open questions of nutrient cycle change in rainforest ecosystems.

\subsection{Acknowledgments}

We thank the support and the sponsorship of German Research Foundation (DFG) in the frame of SFB 552 and the Staff at the Storma Laboratory, Palu, Indonesia. 
5 Brandrodungsbeeinflusster Nährstoffeintrag über Bestandsniederschlag in Zentralsulawesi (Indonesien) - ein Vergleich von Regenwald und Kakaoagroökosystem

Der Artikel „Brandrodungsbeeinflusster Nährstoffeintrag über Bestandsniederschlag in Zentralsulawesi (Indonesien) - ein Vergleich von Regenwald und Kakaoagroökosystem“ wird in der Zeitschrift GeoÖko veröffentlicht. 


\section{Brandrodungsbeeinflusster Nährstoffeintrag über}

\section{Bestandsniederschlag in Zentralsulawesi (Indonesien) - ein Vergleich von Regenwald und Kakaoagroökosystem}

\section{Influence of bulk deposition caused by slash-and-burn to Throughfallinput in Central Sulawesi (Indonesia) - A comparison of Rainforest and Cakaoplantations}

S. Köhler ${ }^{\text {ab }}$

aLandscape Ecology, Institute of Geography, University of Göttingen, Goldschmidtstr. 5, 37077 Göttingen, Germany

${ }^{b}$ Landscape Ecology and Land Evaluation, University of Rostock, Justus-von-Liebig-Weg 6, 18051 Rostock, Germany

E-Mail: stefan.koehler@uni-rostock.de

Tel.: +49381-4983224

Fax: $+49381-4983220$

\section{Zusammenfassung:}

Nährstofftransporte aus anthropogenen Quellen beeinflussen zunehmend abgelegene Regenwaldstandorte und verändern Nährstoffkreisläufe zu Ungunsten N-limitierter Arten. In Zentral Sulawesi (Indonesien) wurde an drei Standorten mittels ionenbasierter Passivsammler sowohl die Freilanddeposition als auch die Bestandesdeposition für den immergrünen Regenwald wie die Kakaoplantage erfasst. Gemessene Frachten für die Elemente Aluminium, Kalzium, Eisen, Magnesium, Mangan, Natrium und Phosphor waren gering am naturbelassenen Standort Bariri (Regenwald). Für Nitratstickstoff konnten Einträge mit $0,2 \mathrm{~kg} \mathrm{ha}^{-1} \mathrm{a}^{-1}$ unterhalb vergleichbarer Regenwaldstandorte erfasst werden. Bestandesdepositionen lagen im Bereich anderer bisher erfasster Regenwald- und Kakaostandorte. Die durch nahegelegene Brandrodungsflächen beeinflussten Regenwald- und Kakaoflächen zeigten für alle Elemente deutlich höhere Depositionsfrachten. Im Bestand wurde eine deutliche Anreicherung von Stickstoff und Phosphor gemessen. Mit dem Vergleich der Untersuchungsflächen wird deutlich, dass sich die kleinparzellierten traditionellen Brandrodungsflächen nur lokal und nicht weiträumig regional bisher auf den Nährstoffeintrag ins Regenwaldökosystem ausgewirkt haben.

Schlüsselwörter: Freilanddeposition, Bestandesdeposition, Brandrodung, Sulawesi, Regenwald, Kakaoplantage 


\section{Summary:}

Nutrient emission from anthropogenic sources increase and influences permanently remote rainforests. Changed nutrient cycles are in favour for the fewer opponents of manifold species adapted to N-limitation. For an investigation on Central Sulawesi (Indonesia) at three field sites bulkand throughfall deposition were measured with ion exchange resin based passive collectors at rain forests and cacao plantations. For the elements aluminium, calcium, iron, potassium, magnesium, manganese, sodium and phosphorus low depositions were detected at Bariri (Rain forest). For nitrate lower depositions with $0.2 \mathrm{~kg} \mathrm{ha}^{-1} \mathrm{a}^{-1}$ were detected than for other remote areas. Throughfall in rain forest and cacao plantations were in the range of literature described for other rain forest sites. Field sites, influenced by nearby located slash-and-burn activities show higher depositions. Especially phosphorus and nitrogen showed higher input in throughfall depositions. In a comparison it is shown that small holder's practicing traditional slash-and burn certainly influence local forest ecosystems.

Key words: bulk deposition, throughfall, slash-and-burn, Sulawesi, Rainforest, Cacao Plantation

\subsection{Einleitung}

Der vertikale Nährstoffumsatz in tropischen Waldökosystemen unterliegt komplexen Prozessen aufgrund der vielfältigen Interaktionen zwischen atmosphärischen Bedingungen, Stockwerkbau und Artenvielfalt sowie vielfältigen Vegetations-Bodeninteraktionen. Der Nährstofftransfer läuft nach Whitmore (1993) v.a. über vier Pfade ab (Nicklas, 2006).

1. Externer und interner Eintrag durch Freilandniederschlag, Bestandsniederschlag und Stammabfluss

2. Systeminterner Umsatz der Biomasse durch Streufall und Wurzelmasse

3. Nährstoffaufnahme der Pflanze aus dem Boden

4. Austrag von Nährstoffen durch Auswaschung aus dem Boden (leaching)

Anschaulich ist dies in Abb. 5.1 nach Nicklas (2006) dargestellt. Betrachtet wird dabei der ans Wasser gebundene Nährstofftransfer (Bruijnzeel 1991), welcher in das Ökosystem durch Niederschläge eingetragen wird und über internen (Bestandsniederschlag-Bodenwasser-WurzelwasseraufnahmeSpeicherung in Biomasse) sowie externen Wasser- und Nährstoffumsatz (Freilandniederschlag Sickerwasser unterhalb der Durchwurzelungszone - Transpiration) geprägt wird. 


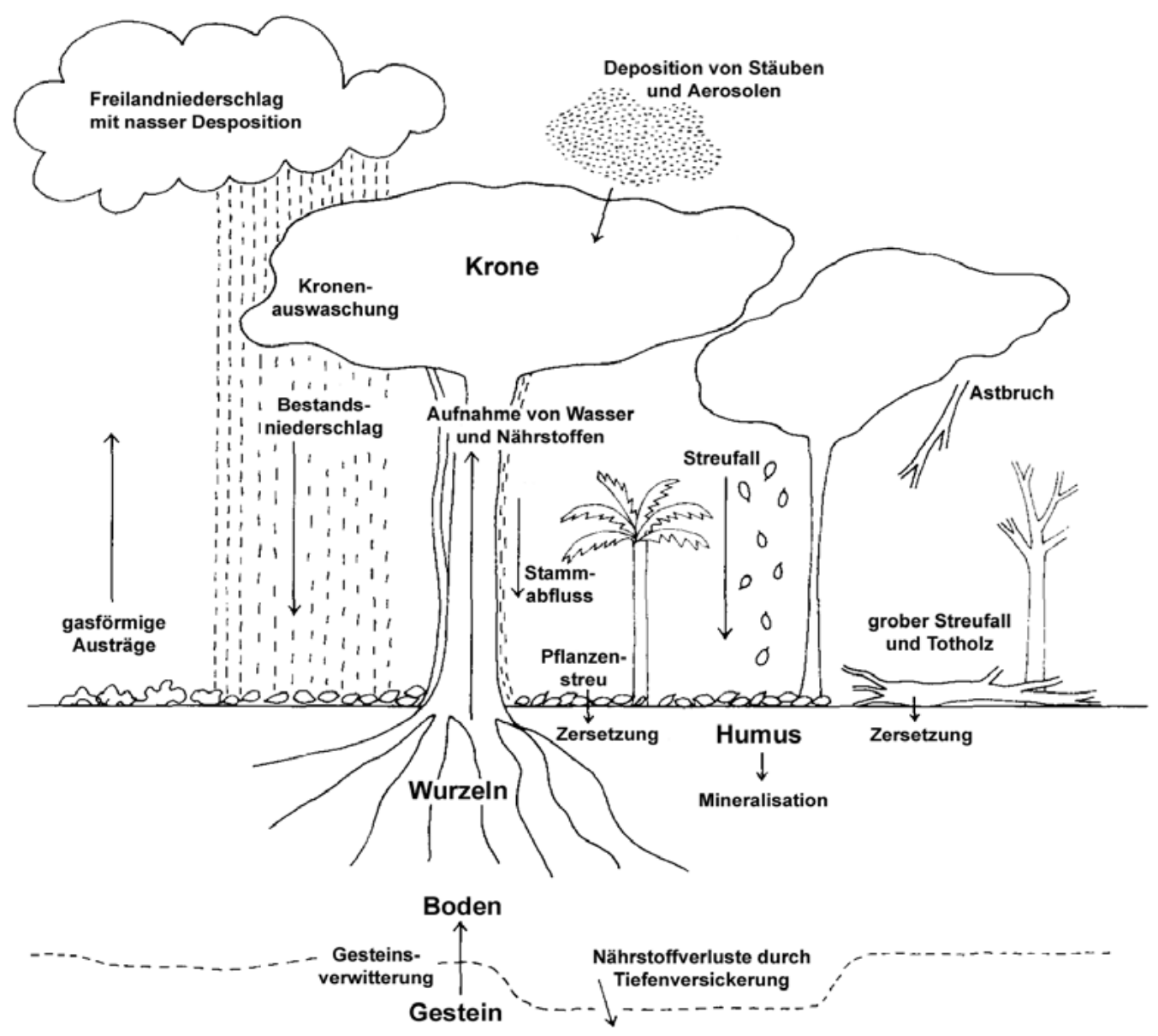

Abb. 5 1: Wasser- und Nährstoffumsatz in Regenwaldökosystemen (verändert nach Proctor 1987; Nicklas 2006)

Fig. 5.1: Water- and nutrient cycles in tropical rain forests (after Proctor 1987; Nicklas 2006)

Für die Nährstoffversorgung der Durchwurzelungszone (Oberboden) stellen dabei der Freiland- bzw. Bestandsniederschlag und der Streufall die wichtigsten Flüsse dar. Im Niederschlag sind bedeutende Mengen gelöster und suspendierter Stoffe enthalten (Herrmann, 1977). Dabei handelt es sich um gelöste Aerosole, gelöste Gase oder Salze, welche von Regentropfen in der Atmosphäre aufgenommen werden. Dieser Niederschlag enthält die sogenannte nasse Deposition, fällt direkt in Niederschlägen zur Erde und wird vor dem Kontakt zu einem Pflanzenbestand als Freilandniederschlag bezeichnet (Abb. 5.1). Als trockene Deposition bezeichnet man feste Stoffe in Form von Aerosolen und kolloidaler Stäube (Abb. 5.1), die sich auf Oberflächen von Pflanzen ablagern und in dieser Form nicht pflanzenverfügbar sind. Stäube der trockenen Deposition werden durch den Kontakt mit Freilandniederschlägen gelöst oder suspendiert und gelangen als Bestandsniederschlag (wash out) zur Bodenoberfläche im Bestand.

Nasse- und trockene Depositionen sind externe Nährstoffeinträge in Ökosysteme und bedeuten einen Zugewinn an Nährstoffen für ein Ökosystem. Während der Passage von Niederschlagswasser kommt es zu einer Anreicherung von Nährstoffen, die aus externen Quellen (Trockendeposition) und internen Quellen (Nährstoffaustausch/-auswaschung aus Blattoberflächen) bestehen. 
Der Bestandsniederschlag stellt durch Eintrag direkt pflanzenverfügbarer Nährstoffe eine der primären Nährstoffquellen im System dar. Durch Störungen des empfindlichen Nährstoffgleichgewichtes, zum Beispiel dem Eintrag erhöhter anthropogener Nährstoffe oder Schadstoffe, werden Nährstoffbalancen verändert und endemische oligotrophe Arten verdrängt.

Häufig sind tropische Regenwälder auf stark verwitterten Böden mit geringen Nährstoffnachlieferungsvermögen anzutreffen (Whitmore 1993). Der Eintrag von Nährstoffen über den Freilandniederschlag stellt eine wichtige Nährstoffquelle für den Bestand dar. Die Dimension solcher Nährstofftransporte ist beachtlich und wird beispielsweise als Staubexport aus der Sahara auf mehr als 200 Millionen Tonnen pro Jahr in Richtung Amazonasbecken geschätzt (Herrmann et al. 2010).

Die starke Heterogenität der weltweiten Depositionsfrachten in Waldökosysteme als Freilandeintrag wird aus Tab. 5.1 ersichtlich. In lateinamerikanische küstennahe Regenwaldstandorte werden jährlich zwischen 6 und $30 \mathrm{~kg} \mathrm{ha}^{-1} \mathrm{a}^{-1}$ Kalzium (Ca) eingetragen, das aufgrund der meist sauren pH-Bodenbedingungen im Boden selbst nicht nachgeliefert werden kann, während in Bergregenwälder die Deposition zwischen 0,8 und 17,5 $\mathrm{kg} \mathrm{ha}^{-1} \mathrm{a}^{-1} \mathrm{Ca}$ liegt. In afrikanischen Waldstandorten ist die Deposition zwischen 6 und $10 \mathrm{~kg} \mathrm{ha}^{-1} \mathrm{a}^{-1} \mathrm{Ca}$ und in asiatischen Regenwäldern zwischen 4 und $20 \mathrm{~kg} \mathrm{ha}^{-1} \mathrm{a}^{-1} \mathrm{Ca}$.

Tab. 5.1: Nährstoffeinträge als Freilanddeposition in tropischen Regenwaldstandorten (kg ha $\left.\mathrm{a}^{-1} \mathrm{a}^{-1}\right)$

Tab. 5.1: Nutrient fluxes in incident rainfall in tropical lowland rainforests $\left(\mathrm{kg} \mathrm{ha}^{-1} \mathrm{a}^{-1}\right)$

\begin{tabular}{l|cccccccc} 
& $\begin{array}{c}\text { altitude } \\
\text { (m a.s.I.) }\end{array}$ & $\begin{array}{c}\text { rainfall } \\
\text { (mm per year) }\end{array}$ & $\mathrm{Ca}$ & $\mathrm{Mg}$ & $\mathrm{K}$ & $\mathrm{Na}$ & $\mathrm{NO}_{3}-\mathrm{N}$ & $\mathrm{PO}_{4}-\mathrm{P}$ \\
\hline 1-4: continental & & & & & & & & \\
Brazil-Manaus (1) & 50 & 2622 & 0.80 & 0.3 & 2.60 & - & 1.40 & 0.07 \\
Brazil-Rio Negro (2) & 90 & 2900 & 2.50 & 0.4 & 0.70 & 2.50 & 0.80 & - \\
Brazil-Rondonia (3) & 143 & 2300 & 17.50 & 1.60 & 8.70 & 3.40 & 0.80 & - \\
Venezuela Gran Sabana (4) & 1300 & 2548 & 0.80 & 1.30 & 2.20 & - & 1.30 & 0.00 \\
5-10: marine & & & & & & & & \\
Brazil-Rio Grande do Sul (5) & 175 & 1588 & 11.20 & 3.70 & 18.60 & 42.50 & 3.20 & 8.9 (P2O5) \\
Brazil-Mata Atlantica (6) & 10 & 2235 & 5.60 & 1.60 & 6.40 & 75.20 & - & - \\
Costa Rica SW (7) & 70 & 5810 & 20.00 & 5.40 & 6.20 & 6.90 & 2.00 & 0.4 \\
Costa Rica Monteverde (8) & 1500 & 3191 & 5.80 & 2.40 & 3.00 & 20.50 & 1.70 & 0.05 \\
Panama Cord.Central (9) & 1200 & 3510 & 27.90 & 4.10 & 13.50 & 63.50 & - & 0.7 \\
Puerto Rico (9) & 425 & 3750 & 21.80 & 4.90 & 18.20 & 57.20 & - & - \\
Kamerun Korup NP(10) & 150 & 5370 & 9.30 & 5.30 & 7.80 & - & 1.50 & 1.10 \\
Nigeria Ife-Ife (11) & 215 & 1413 & 5.80 & 2.50 & 5.40 & 15.70 & 10.40 & 1.70 \\
Malaysia (9) & 870 & 2700 & 4.00 & 1.20 & 4.00 & - & 5.00 & 0.10 \\
Sulawesi Lore Lindu National Park & & & & & & & & \\
(12) & $629-1375$ & $\sim 2500$ & $4.6-19-4$ & $0.9-3.2$ & $1.6-10.6$ & $6.8-21.1$ & $0.1-2.7$ & $0.6-2.0$
\end{tabular}

Schroth et al. 2001; (2) Filoso et al. (1999); (3) Germer et al. (2007); (4) Dezzo and Chacon (2006); (5) Calil et al. (2010); (6) Scheer (2011); (7) Hofhansel et al. (2011); (8) Hafkenscheid (2002); (9) Ashagrie and Zech (2010), (10) Chuyong et al.(2004), (11) Oziegbe et al.(2011),(12) Köhler et al. 2012b nach G. Gerold, persönliche Mitteilung 
Kalium (K), ein Element, das einer sehr starken Bodenauswaschung unterliegt, wird in küstennahen Standorten mit 4-19 $\mathrm{kg} \mathrm{ha}^{-1} \mathrm{a}^{-1}$ eingetragen. In kontinental geprägten Standorten werden Frachten von 0,7 bis 2,6 $\mathrm{kg} \mathrm{ha}^{-1} \mathrm{a}^{-1}$ verzeichnet. Tab. 5.1 zeigt deutlich, dass küstennahe Standorte einen höheren Nährstoffeintrag gegenüber kontinentalen Inlandstandorten verzeichnen. Hervorzuheben sind die extrem niedrigen Freilanddepositionen der Kernnährstoffe $N$ und $P$ in kontinentalen Regenwaldgebieten wie auch auf niedrigem Niveau in maritim beeinflussten Regenwäldern (Ausnahme: $\mathrm{NO}_{3}-\mathrm{N}$ Nigeria - wahrscheinlich brandrodungsbeeinflusst).

Werden natürliche Frachten mit anthropogen verursachten Nährstoffen angereichert, kann es zu einer Erhöhung von Nährstofffrachten in sensible Ökosysteme kommen. Beispielsweise Nitratfrachten $\left(\mathrm{NO}_{3}-\mathrm{N}\right.$ ) über $0,6 \mathrm{~kg} \mathrm{ha}^{-1} \mathrm{a}^{-1}$ (Ayers et al. 2000) deuten auf einen antropogenen Eintrag hin.

Kenntnisse über den Nährstoffeintrag in Regenwaldökosysteme, deren Transportwege im Bestand sowie Verluste durch Auswaschung (leaching) sind grundlegende Kenngrößen, um die Funktion dieser Ökosysteme zu beurteilen und Veränderungen zu beschreiben. Ähnliches gilt für Argarökosysteme, die an tropischen Standorten in unmittelbarer Nachbarschaft angesiedelt und in Wechselwirkung mit den Waldökosystemen stehen.

Die vorliegende Arbeit betrachtet Bestandeseinträge zweier unterschiedlicher Regenwaldökosysteme, sowie einer Kakaoplantage in Zentralsulawesi. Dabei wird davon ausgegangen, dass Nährstoffeinträge aus natürlichen Quellen und ähnlichem maritimem Einfluss ähnliche Depositionsmengen an verschiedenen Standorten einer Region zeigen. Besondere Beachtung findet der Eintrag von Nitrat-Stickstoff $\left(\mathrm{NO}_{3}-\mathrm{N}\right)$ sowie Phosphor $(\mathrm{P})$, da beide Stoffe in natürlichen Systemen meist nur gering eingetragen werden (z.B. Malaysia 0,6 kg ha- $\mathrm{NO}_{3}-\mathrm{N}$ (Ayers et al. 2002), s. auch Tab. 5.1 kontinentale Standorte) und damit die limitierenden Nährstoffe im tropischen Waldökosystem darstellen.

\subsection{Material und Methoden}

\subsubsection{Untersuchungsgebiet}

Durchgeführt wurde die Untersuchung in Sulwaesi, einer Insel des Indonesischen Archipels, im Rahmen des Sonderforschungsbereiches 552. Die Versuchsflächen sind an der Randzone bzw. einer Enklave in dem $2310 \mathrm{~km}^{2}$ großen Lore Lindu National Park gelegen, der sich über Höhenlagen zwischen 600 und $2600 \mathrm{~m}$ erstreckt. Im Mittel liegen die jährlichen Niederschlagsummen bei $2500 \mathrm{~mm}$ mit einer Tagesdurchschnittstemperatur von $24.5^{\circ} \mathrm{C}$ (Kleinhans 2003), mit einer hohen annuellen Schwankung zwischen Trockenjahren (2004: $2044 \mathrm{~mm}$ nach de Vries, persönliche Mitteilung) und Feuchtjahren (2007: 3452 nach Gutzler, persönliche Mitteilung) Der Jahresgang der Niederschläge, mit Niederschlägen über $100 \mathrm{~mm}$ in jedem Monat, ist charakterisiert durch zwei innertropische monsunale Maxima im April und Oktober (Leemhuis 2005). 


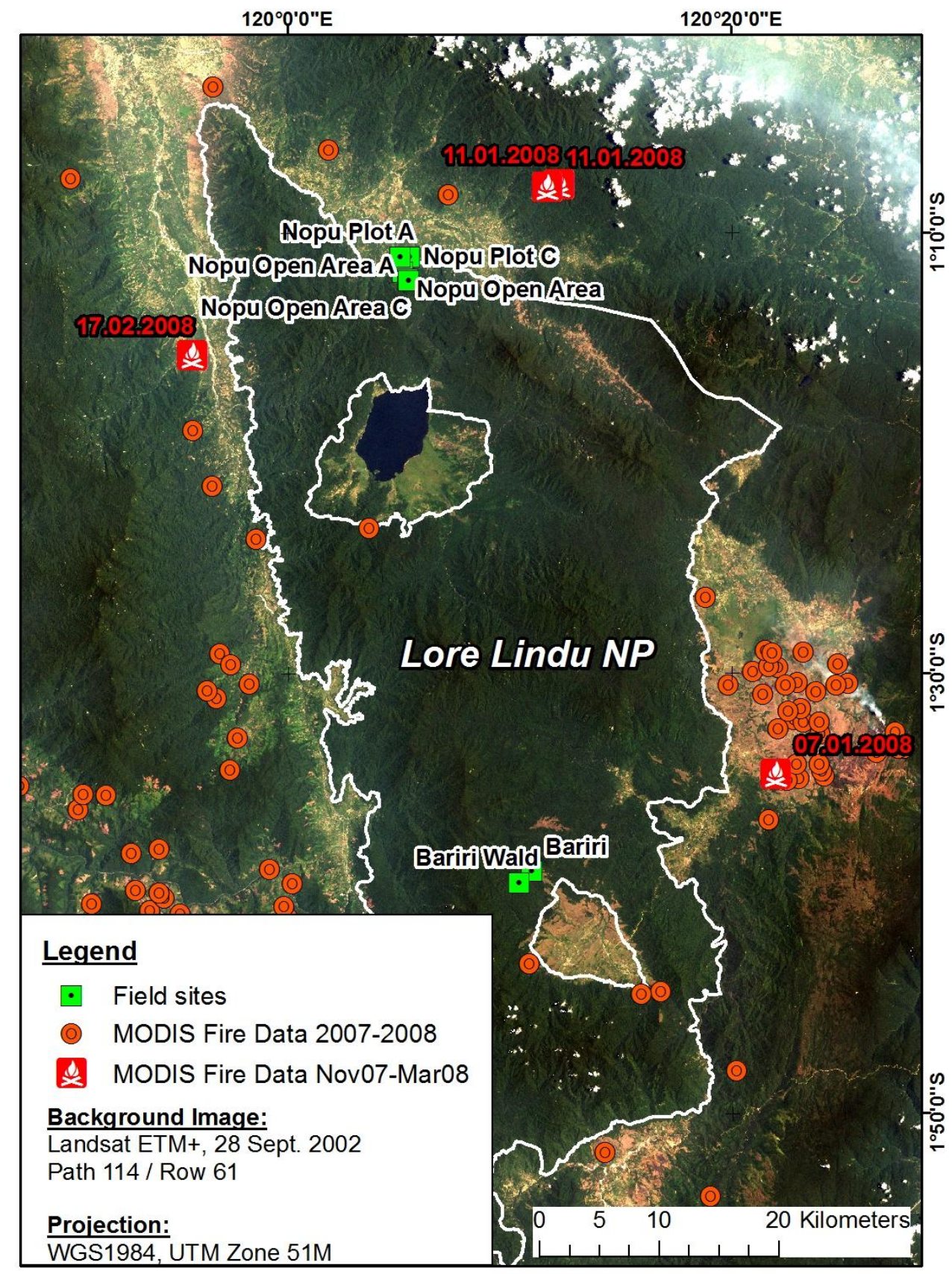

Abb. 5.2: Übersichtskarte des Lore Lindu National Parks mit den Standorten für Freiland- und Bestandesdepositionsmessung sowie Brandereignissen im Untersuchungszeitraum (2007 und 2008) in Zenral Sulawesi. Die Daten wurden dem "LANCE FIRMS MODIS Fire/Hotspot" Archive entnommen unter http://earthdata.nasa.gov/firms (zugriff: 08 Mai 2012) . Eine weiterführende Beschreibung der Modis Feuerdaten ist unter Giglio et al. (2004) verfügbar (Karte nach S. Erasmi, persönliche Mitteilung).

Fig. 5.2: Overview of field sites for throufall and buld depositionmesurements and fire hotspots in Central Sulawesi, Indonesia for the time period 2007-2008. Data about fire hotspots are taken from the "LANCE FIRMS MODIS Fire/Hotspot" archive at http://earthdata.nasa.gov/firms (last access 08 May 2012). Details about the MODIS Fire product are available from Giglio et al. (2003).

Durch kleinbäuerliche Brandrodung in den Randzonen des Parks werden permanent landwirtschaftliche Nutzflächen hinzu gewonnen, die zunächst für annuelle Kulturen, vor allem 
Maisanbau, genutzt werden und nach 2-3 Jahren in einen permanenten Anbau von Kakao übergehen.

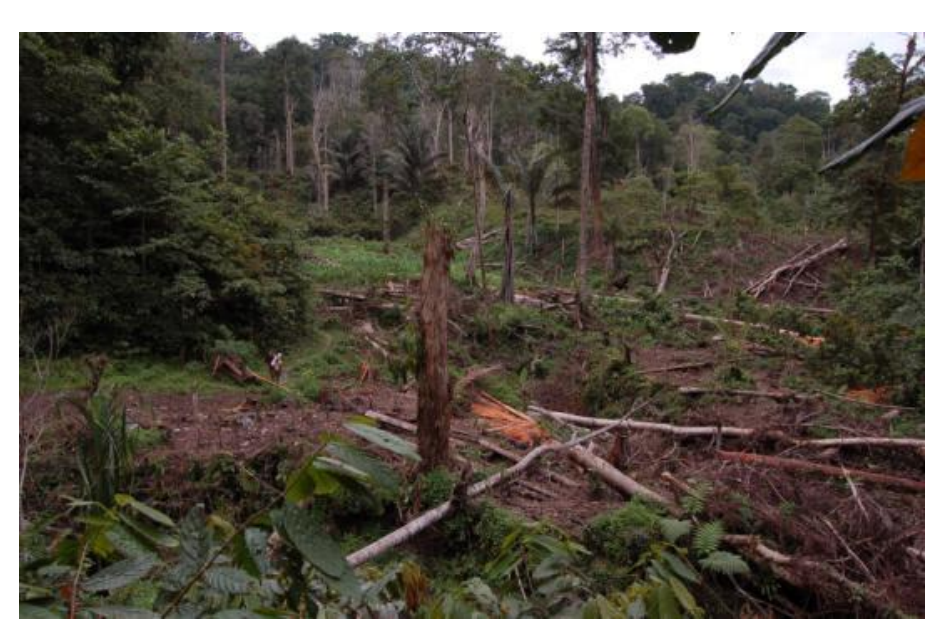

Abb.5 3: Brandrodungsfläche unterhalb des Versuchsstandortes Nopu Plot C

Fig. 5.3: Slash and burn area near the field site Nopu Plot $C$

\subsubsection{Untersuchungsflächen und Versuchsdesign}

Zur Erfassung des Bestandsniederschlages wurden drei Versuchsplots eingerichtet. Die Versuchsplots Nopu Plot A und Nopu Plot $C$ sind nahe dem Dorf Nopu gelegen und bezeichnen Versuchsflächen des SFB-Teilprojekt B2 (Gutzler2011). Bei Nopu Plot A handelt es sich um eine 17 jährige Kakaoplantage auf einer Höhe von $639 \mathrm{~m}$ ü. NN. Die Plantage ist am Hangfuß eines Bergrückens gelegen und befindet sich am Rande des Lore Lindu Nationalparks (Abb. 5.4). Nopu Plot $C$ bezeichnet einen Regenwaldplot in 1082 m. ü. NN (Abb. 5.5). Der Plot liegt auf einer nordexponierten Hügelkuppe im oberen Bereich des Bergrückens. Unterhalb des Plots, auf einer Hanghöhe von ungefähr $920 \mathrm{~m}$ über NN, wurden während der Untersuchung starke Brandrodungsaktivitäten (Abb. 5.3) durchgeführt, bei der die gerodete Biomasse verbrannt wurde.
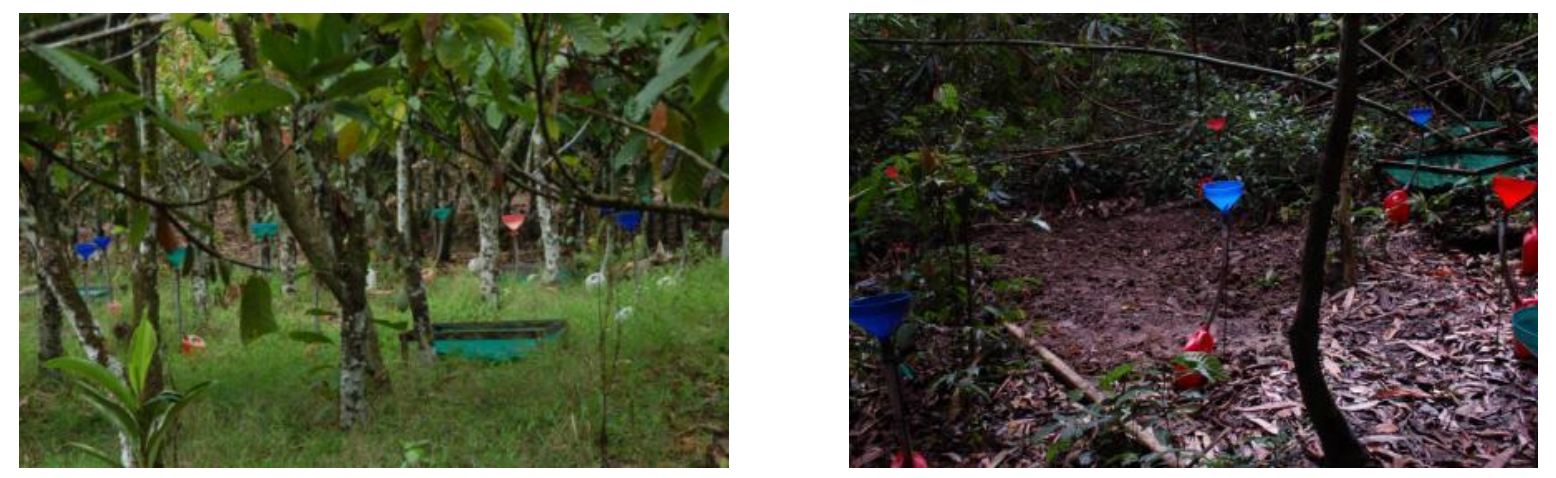

Abb. 54 Kakaoplantage Nopu Plot A und Abb. 55 Waldstandort Nopu Plot C mit Versuchsanlage des Partnerprojektes B2 Fig. 5.4 and Fig. 5.5: Cacao plantation and forest site with measurement equipment of our partner project B2

Die Größe der Versuchsflächen an den Standorten Nopu Plot A und Nopu Plot C betrug $10 \times 10 \mathrm{~m}$. Auf diesen Flächen wurden jeweils 20 IER-Kollektoren, beschrieben in Köhler et al. (2012), an festen 
Standpunkten installiert. Die Erfassung der Messwerte erfolgte in der Zeit von Anfang Mai 2007 bis Ende Mai 2008. Der Wechsel der lonentauscher erfolgte in einem 14-tägigen Intervall.

Als Referenz wurde der in einer Höhe von $1431 \mathrm{~m}$ ü. NN eingerichtete Waldstandort (Bariri Wald) nahe der Ortschaft Bariri genutzt (Abb. 5.6). Im Gegensatz zu den Flächen in Nopu findet am Standort Bariri Wald keine selektive Holzentnahme statt, da durch die Entfernung zum Dorf ein Holztransport zu aufwendig ist. Der Standort, durch seine abgeschnittene Kessellage, wird nicht durch umliegende landwirtschaftliche Nutzung beeinflusst. Die Untersuchungsflächen sind in einem Primärwald eines immergrünen Laubwaldes gelegen und verteilen sich um einen $70 \mathrm{~m}$ hohen Turm zur Klimadatenerfassung (SFB Teilprojekt B7).

Tab. 5.2: Position und Eigenschaften der Untersuchungsstandorte

Tab. 5.2: Position and attributes of the field sites

\begin{tabular}{|c|c|c|c|c|c|}
\hline & Position S & Position E & $\begin{array}{c}\text { Höhe } \\
\text { [ m. ü. NN.] }\end{array}$ & $\begin{array}{c}\text { Bäume } \\
\text { pro ha } \\
(\mathrm{dbh} \geq 10 \mathrm{~cm})\end{array}$ & $\begin{array}{l}\text { Blattflächen- } \\
\text { index } \\
\left(\text { LAI } \mathrm{m}^{2} \mathrm{~m}^{-2}\right)\end{array}$ \\
\hline Nopu Plot A & $01^{\circ} 11.081^{\prime}$ & $120^{\circ} 5076^{\prime}$ & 639 & 1500 & 4,9 \\
\hline Nopu Plot C & $01^{\circ} 12.157^{\prime}$ & $120^{\circ} 5.472^{\prime}$ & 1082 & 311 & 5,1 \\
\hline $\begin{array}{l}\text { Bariri Wald } \\
\text { Freilandflächen }\end{array}$ & $01^{\circ} 39.517^{\prime}$ & $120^{\circ} 10403^{\prime}$ & 1431 & 511 & 6,5 \\
\hline Nopu Open Area A & $01^{\circ} 11.099^{\prime}$ & $120^{\circ} 5025^{\prime}$ & 641 & - & - \\
\hline Nopu Open Area C & $01^{\circ} 12.067^{\prime}$ & $120^{\circ} 5.439^{\prime}$ & 958 & - & - \\
\hline Nopu Open Area & $01^{\circ} 11.778^{\prime}$ & $120^{\circ} 5.176$ & 837 & - & - \\
\hline Bariri & $01^{\circ} 38.991^{\prime}$ & $120^{\circ} 10.982$ & 1370 & - & - \\
\hline
\end{tabular}

nach Gutzler (2011) und Ibrom (2008), verändert und ergänzt

An 12 Messstellen am Standort Bariri-Wald wurden die Messungen an einer bereits bestehenden Anlage zur Erfassung der Wassermengen im Bestand durchgeführt. Das Wasser des Bestandesniederschlags wurde in einer $0,274 \mathrm{~m}^{2}$ großen Rinne aufgefangen und in 100 । Kunststofffässern gesammelt. Die mit lonentauschern gefüllten Sammelröhren wurden am Fasseintritt befestigt, so dass alles Regenwasser den Sammler passieren musste (Abb. 5.6). Zusätzlich wurden Untersuchungen zum Stammablauf gemacht. Anfallendes Wasser aus dem Stammablauf wurde im gleichen System beprobt und dem Flächeneintrag zugeschlagen. 


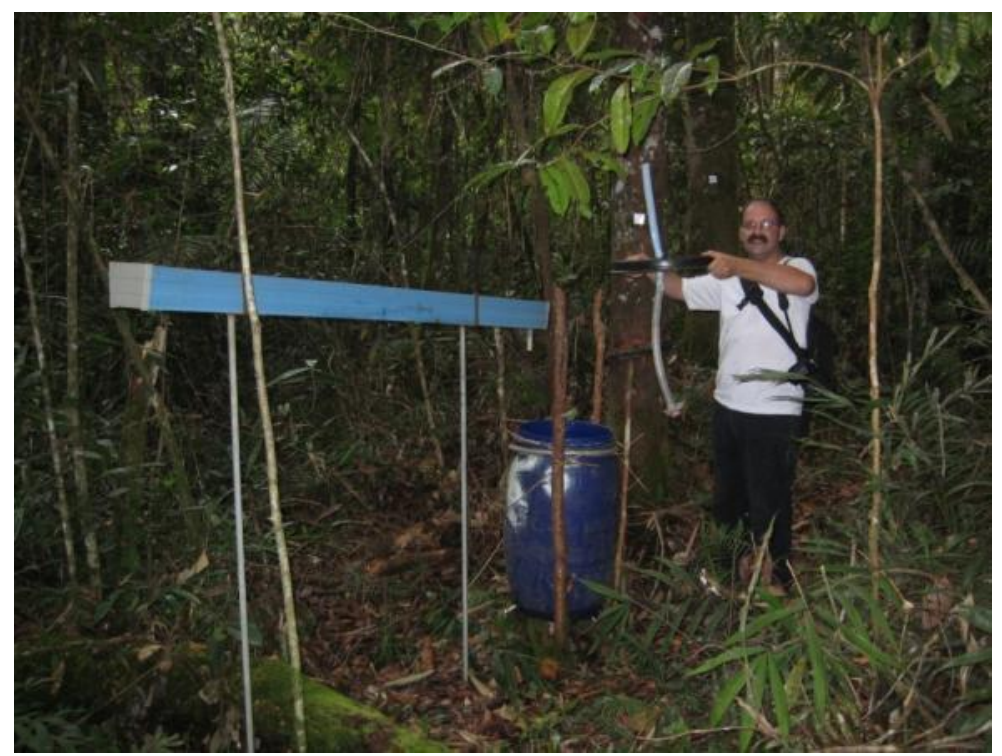

Abb. 5.6: Passivsammler für Bestandsniederschlag am Standort Bariri Regenwald

Fig. 5.6: Passive collector system for throughfall deposition at the site Bariri rainforest

An allen drei Standorten wurden entsprechend Messstellen zur Erfassung des Freilandniederschlages mit jeweils drei Wiederholungen eingerichtet. Zunächst wurde im Bereich der Bestandsmessstellen Nopu Plot A und Nopu Plot $C$ nur eine zentrale Freilandsniederschlagsmessstelle (Nopu Open Area: $837 \mathrm{~m}$ ü. NN) installiert. Im August 2007 wurde zur Erfassung der kleinräumigen Niederschlagsverteilung in Nopu in unmittelbarer Nähe der Bestandsmessstellen weitere Freilandsniederschlagsmessstellen (Nopu Open Area A (641 m über NN) und Nopu Open Area C (958 m über NN)) eingerichtet. Im Verlauf des Jahres 2008 zeigte sich, dass die eingetragenen Depositionen in der Freilandmessstelle Nopu Open Area deutlich geringer waren als die der zusätzlich installierten Freilandmessstellen. Im Ergebnisteil werden die Jahreswerte für die Freilandsniederschlagsmessstelle Nopu Open Area ebenfalls gezeigt und beziehen sich auf den Zeitraum Juni 2007 bis Mai 2008. Berechnungen zur Anreicherung von Nährstoffen im Bestandesniederschlag beziehen sich auf die näher gelegenen Messstellen und den kürzeren neunmonatigen Zeitraum September 2007 bis Mai 2008.

Aufgrund unterschiedlicher Sammeloberflächen der verwendeten Messsysteme in den unterschiedlichen Bestandesniederschlagserfassungen ist die Flächenabdeckung und damit die Auflösung der Systeme verschieden. Entsprechend der Auffangmessfläche wurden die Elementdepositionen auf $\mathrm{g} \mathrm{m}^{-2}$ berechnet und über die Anzahl der Sammler Mittelwert und Standardabweichung berechnet.

Durch Nährstoffanreicherung im Bestandesniederschlag wurden hohe Nährstofffrachten erwartet. Um eine vollständige Belegung der Austauschkapazität zu vermeiden, wurden alle Sammler in den Bestandesanlagen 14-tägig getauscht. Für die Standorte in Nopu wurden im Oktober 2007 krankheitsbedingt keine Proben genommen und die Sammler erst im November gewechselt. Die Depositionsgehalte wurden anteilig für den Oktober berechnet. 


\subsection{Ergebnisse}

An den drei Standorten wurden sehr unterschiedliche Mengen für die deponierten Elemente Aluminium (Al), Kalzium (Ca), Eisen (Fe), Kalium (K), Magnesium (Mg), Mangan (Mn), Natrium ( $\mathrm{Na}$ ), Nitratstickstoff $\left(\mathrm{NO}_{3}-\mathrm{N}\right)$ und Phosphor $(\mathrm{P})$ im Bestandesniederschlag erfasst (Tab. 5.3). Für alle gemessenen Elemente wurden die geringsten Werte in Bariri Wald, mit weniger als $0,1 \mathrm{~kg} \mathrm{ha}^{-1} \mathrm{a}^{-1}$ für $\mathrm{NO}_{3}-\mathrm{N}$ und 26,6 kg ha- ${ }^{-1} \mathrm{a}^{-1}$ für $\mathrm{K}$, gemessen (Tab. 5.3). Deutlich höhere Depositionsmengen wurden im Kakaobestand am Standort Nopu Open Area A mit 1,5- bis 10-fach höheren Werten erfasst. Für $\mathrm{NO}_{3}-\mathrm{N}$ zeigte sich ein 15-fach höherer Bestandeseintrag als in Bariri Wald (Tab. 5.3). Die Einträge im Regenwaldstandort Nopu Plot $\mathrm{C}$ überstiegen die Einträge in der Kakaoplantage leicht für die Elemente $\mathrm{Al}$ und $\mathrm{Fe}$, für $\mathrm{NO}_{3}-\mathrm{N}$ wurde ein etwas geringerer Eintrag verzeichnet. Für alle anderen Elemente wurden zwei- bis fünffach höhere Einträge im Waldstandort Nopu Plot C erfasst (Tab. 5.3). Die Standorte zeigen damit eine Regelhaftigkeit in der Zunahme von deponierten Elementen von Bariri (ungestörter Regenwald) über Nopu Plot A (Kakaoplantage) zu Nopu Plot C (gestörter Regenwald). Einzig abweichend von dieser Regel verhält sich die Deposition von $\mathrm{Mn}$ mit deutlich höheren Gehalten in Bariri und den geringsten Gehalten in Nopu Plot C. In den Jahresdepositionen der Freilandmessstellen wurden leicht höhere Werte in der zentralen Messstelle Nopu Open Area A gegenüber Bariri erfasst (Tab. 5.3).

Tab. 5.3: Jährliche Bestandsdeposition und Freilanddeposition der unterschiedlichen Standorte in $\mathrm{kg}^{*} \mathrm{ha}^{-1}$

Tab. 5.3: Annual deposition for bulk- and through fall deposition at the different sites ( $\left.\mathrm{kg} \mathrm{ha}^{-1}\right)$

\begin{tabular}{|c|c|c|c|c|c|c|c|c|c|c|}
\hline & $\begin{array}{l}\text { Bariri Wald } \\
\text { (Regenwald) }\end{array}$ & & $\begin{array}{c}\text { Bestand } \\
\text { Nopu Plot A } \\
\text { (Kakao) }\end{array}$ & & $\begin{array}{l}\text { Nopu Plot C } \\
\text { (Regenwald) }\end{array}$ & & Bariri & & $\begin{array}{c}\text { Freiland } \\
\text { Nopu Open Ar }\end{array}$ & \\
\hline & $\mathrm{kg} \mathrm{ha}^{-1} \mathrm{a}^{-1}$ & Stab. & $\mathrm{kg} \mathrm{ha} a^{-1} \mathrm{a}^{-1}$ & Stab. & $k g h a^{-1} a^{-1}$ & Stab. & $k g h a^{-1} a^{-1}$ & Stab. & $\mathrm{kg} \mathrm{ha}^{-1} \mathrm{a}^{-1}$ & Stab. \\
\hline $\mathrm{Al}$ & 0.2 & 0.0 & 1.1 & 0.6 & 1.2 & 0.3 & 1.2 & 0.2 & 1.1 & 0.1 \\
\hline $\mathrm{Ca}$ & 13.7 & 4.1 & 21.1 & 6.4 & 49.0 & 14.8 & 11.7 & 1.3 & 13.7 & 2.6 \\
\hline $\mathrm{Fe}$ & 0.1 & 0.0 & 0.8 & 0.2 & 1.0 & 0.4 & 0.6 & 0.1 & 0.7 & 0.1 \\
\hline K & 26.6 & 7.9 & 63.8 & 51.1 & 169.2 & 56.5 & 6.6 & 2.7 & 8.3 & 1.4 \\
\hline $\mathrm{Mg}$ & 4.0 & 1.2 & 8.1 & 5.7 & 14.1 & 3.8 & 1.3 & 0.3 & 2.2 & 0.3 \\
\hline $\mathrm{Mn}$ & 0.4 & 0.0 & 0.3 & 0.1 & 0.1 & 0.0 & 0.2 & 0.1 & 0.1 & 0.1 \\
\hline $\mathrm{Na}$ & 3.4 & 0.6 & 24.8 & 3.5 & 28.9 & 2.8 & 19,5 & 1.2 & 17.1 & 2,1 \\
\hline $\mathrm{NO}_{3}-\mathrm{N}$ & 0.0 & 0 & 0.6 & 0.1 & 0.6 & 0.1 & 0,4 & 0.1 & 1.4 & 0.6 \\
\hline$P$ & 0.6 & 0.1 & 4.7 & 3.8 & 21.2 & 9.1 & 1.0 & 0.3 & 1.1 & 0.2 \\
\hline
\end{tabular}

Um eine differenziertere Bewertung der Bestandesdeposition in Bezug auf die Freilanddeposition zu ermöglichen, wurden im September 2007 bis Juni 2008 in nächster Nähe zu den Nopu Standorten Messstellen für die Freilanddeposition errichtet. Im Vergleich der Freilandmessstellen ergab sich, dass die Station Nopu Open Area geringere Werte zeigte als die direkt den Versuchsplot zugeordneten Freilandmessstellen Nopu Open Area A und Nopu Open Area C (Tab. 5.4). 


\begin{tabular}{|c|c|c|c|c|c|c|c|c|c|}
\hline$\frac{\dot{\pi}}{\dot{\omega n}}$ & & 웅 & $\stackrel{\text { S }}{\stackrel{\sim}{~}}$ & $\stackrel{N}{\stackrel{N}{N}}$ & & $\frac{9}{5}$ & $\stackrel{\circ}{\circ}$ & 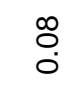 & స̃ \\
\hline 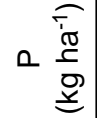 & & 象 & $\stackrel{\mathscr{P}}{\stackrel{m}{m}}$ & $\begin{array}{l}\stackrel{+}{L} \\
0 \\
0\end{array}$ & & $\begin{array}{c}\bar{R} \\
0\end{array}$ & $\stackrel{m}{\rightleftarrows}$ & ๙ั & 只 \\
\hline$\frac{\dot{\pi}}{\dot{n}}$ & & ס̊. & $\frac{0}{\circ}$ & 웅. & & $\begin{array}{l}\mathscr{0} \\
\stackrel{0}{0}\end{array}$ & $\stackrel{\circ}{\check{r}}$ & ô. & 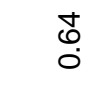 \\
\hline 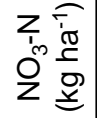 & & $\begin{array}{l}0 \\
0 \\
0 \\
0\end{array}$ & 웅 & $\begin{array}{l}0 \\
\tilde{0} \\
0\end{array}$ & & 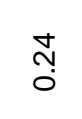 & $\stackrel{?}{\stackrel{N}{N}}$ & $\stackrel{\bar{N}}{\text { N }}$ & 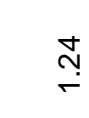 \\
\hline$\frac{\dot{0}}{\dot{\omega n}}$ & & ?ִ & $\stackrel{\infty}{\stackrel{\infty}{\infty}}$ & $\stackrel{5}{i}$ & & $\begin{array}{l}\text { N. } \\
\text { ᄋ̆ }\end{array}$ & 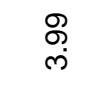 & 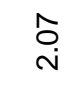 & $\stackrel{N}{\stackrel{N}{r}}$ \\
\hline 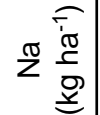 & & r) & $\begin{array}{l}\stackrel{+}{\mathrm{N}} \\
\text { Nat }\end{array}$ & $\begin{array}{l}\infty \\
\infty \\
\stackrel{n}{n}\end{array}$ & & $\stackrel{m}{\stackrel{\infty}{\infty}}$ & ल̄ & $\begin{array}{l}\hat{o} \\
\stackrel{\Gamma}{\circ}\end{array}$ & $\begin{array}{l}\dot{U} \\
\dot{+}\end{array}$ \\
\hline$\frac{\dot{\pi}}{\tilde{\omega}}$ & & $\frac{0}{0}$ & ô. & ס̊ & & $\begin{array}{l}\mathscr{2} \\
0 \\
0\end{array}$ & Oֶ. & $\stackrel{L}{\circ}$ & 饴 \\
\hline 焉 & & ָָ̃ & $\frac{\infty}{0}$ & $\bar{\sigma}$ & & ô & $\stackrel{8}{\circ}$ & $\frac{0}{0}$ & 웅 \\
\hline$\frac{\dot{\pi}}{\dot{\infty}}$ & & \begin{tabular}{l}
$\infty$ \\
\hdashline \\
0 \\
0
\end{tabular} & $\stackrel{\stackrel{P}{+}}{\stackrel{\leftrightarrow}{+}}$ & $\stackrel{m}{\stackrel{m}{N}}$ & & ণิ & ọ & 웅. & ֶָ \\
\hline 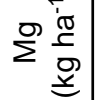 & & لָ & $\begin{array}{l}\stackrel{\leftrightarrow}{1} \\
\dot{0}\end{array}$ & $\begin{array}{l}\bar{\infty} \\
\infty \\
\infty\end{array}$ & & $\begin{array}{l}\stackrel{8}{\infty} \\
\stackrel{0}{0}\end{array}$ & $\stackrel{N}{\stackrel{N}{r}}$ & চ্ & $\stackrel{\widehat{\infty}}{\stackrel{\infty}{r}}$ \\
\hline $\begin{array}{l}\frac{\dot{0}}{\tilde{D}} \\
\dot{\omega}\end{array}$ & & 离 & $\begin{array}{l}\text { N̦ } \\
\text { †े }\end{array}$ & $\begin{array}{l}\stackrel{\wp}{q} \\
\stackrel{g}{q}\end{array}$ & & 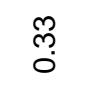 & $\stackrel{\stackrel{a}{d}}{\mathrm{~N}}$ & ఫ̊ & 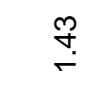 \\
\hline 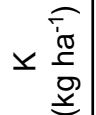 & & مُ & $\begin{array}{l}\tilde{O} \\
\text { ळे }\end{array}$ & $\begin{array}{l}\stackrel{p}{m} \\
\stackrel{m}{m}\end{array}$ & & $\stackrel{\stackrel{N}{N}}{N}$ & $\stackrel{\mathscr{P}}{\stackrel{+}{r}}$ & $\begin{array}{c}\bar{N} \\
\infty\end{array}$ & $\stackrel{+}{\Gamma}$ \\
\hline$\frac{\dot{m}}{\dot{m}}$ & & o. & $\stackrel{\infty}{\circ}$ & $\stackrel{\mathscr{m}}{0}$ & & $\stackrel{+}{\circ}$ & ô. & $\begin{array}{l}8 \\
0 \\
0\end{array}$ & $\sigma_{0}$ \\
\hline 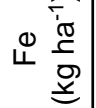 & & $\hat{0}$ & $\stackrel{8}{\circ}$ & $\begin{array}{l}R \\
\stackrel{R}{0}\end{array}$ & & $\stackrel{\mathfrak{Y}}{0}$ & ֻొ & 茵 & 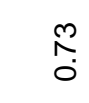 \\
\hline$\frac{\dot{0}}{\dot{\omega n}}$ & & j. & مُ & $\stackrel{\sim}{\stackrel{\infty}{\infty}}$ & & $\stackrel{\dddot{پ}}{\check{r}}$ & $\stackrel{\text { I }}{\text { ì }}$ & 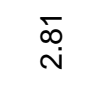 & i্ল \\
\hline 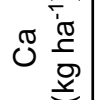 & & 8 & 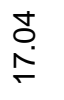 & $\frac{\mathscr{o}}{\frac{\infty}{m}}$ & & $\begin{array}{l}\overline{1} \\
\dot{0}\end{array}$ & $\begin{array}{l}\stackrel{\mathscr{N}}{\mathrm{N}} \\
\stackrel{\sim}{\sim}\end{array}$ & 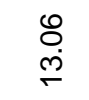 & ô \\
\hline$\frac{\dot{\sigma}}{\dot{\omega}}$ & & 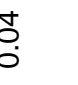 & 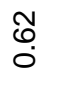 & $\stackrel{\substack{n \\
0}}{0}$ & & م્ّ̣ & $\frac{⿱}{0}$ & $\frac{⿱}{\circ}$ & $F_{0}^{\circ}$ \\
\hline 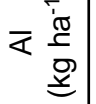 & & $\frac{N}{0}$ & $\begin{array}{l}\stackrel{0}{2} \\
\stackrel{0}{0}\end{array}$ & $\stackrel{\infty}{\stackrel{\infty}{\mathfrak{0}}}$ & & $\begin{array}{l}\infty \\
\infty \\
0 \\
0\end{array}$ & $\stackrel{ }{\leftarrow}$ & $\begin{array}{l}\overline{0} \\
0\end{array}$ & $\underset{r}{8}$ \\
\hline & 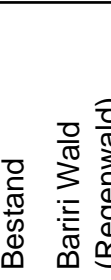 & 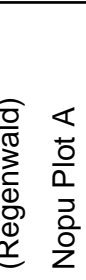 & 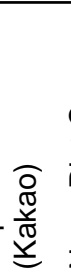 & 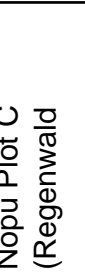 & 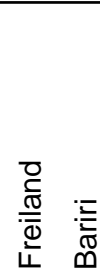 & 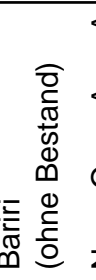 & 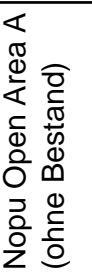 & 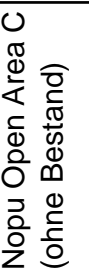 & 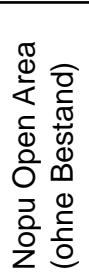 \\
\hline
\end{tabular}

Tab. 5.4: Bestandesdeposition und Freilanddeposition in Bariri und Nopu (9 Monate in kg ha $\mathrm{a}^{-1}$ )

Tab. 5.4: Troughfall and incident rainfall deposition in Bariri and Nopu (9 month $\mathrm{kg} \mathrm{ha}^{-1} \mathrm{a}^{-1}$ ) 
Bei den über neun Monate akkumulierten Bestandesdepositionen wurden die bereits beschriebenen Trends in der Depositionsmenge wieder gefunden (Abb. 5.7). Im Gegensatz dazu ergaben sich die höchsten Depositionen im Freilandniederschlag an der Messstelle Nopu Open Area A, welche im Tal unterhalb der Brandrodungsfläche gelegen ist (Tab. 5.4). Leicht geringere Einträge im Freiland wurden an der Messstelle Nopu Open Area C erfasst, welche oberhalb der Brandrodungsfläche in der Nähe der Waldfläche Nopu Plot C gelegenen ist. Die geringsten Einträge im Freiland wurden in Bariri gemessen (Abb. 5.7, Tab. 5.4). Abweichend zu den anderen Nährstoffen ist auch hier die Deposition von $\mathrm{Mn}$ im Bestandesniederschlag, Bariri die höchsten und in Nopu Plot $\mathrm{C}$ die geringsten sind. (Tab. 5.4)
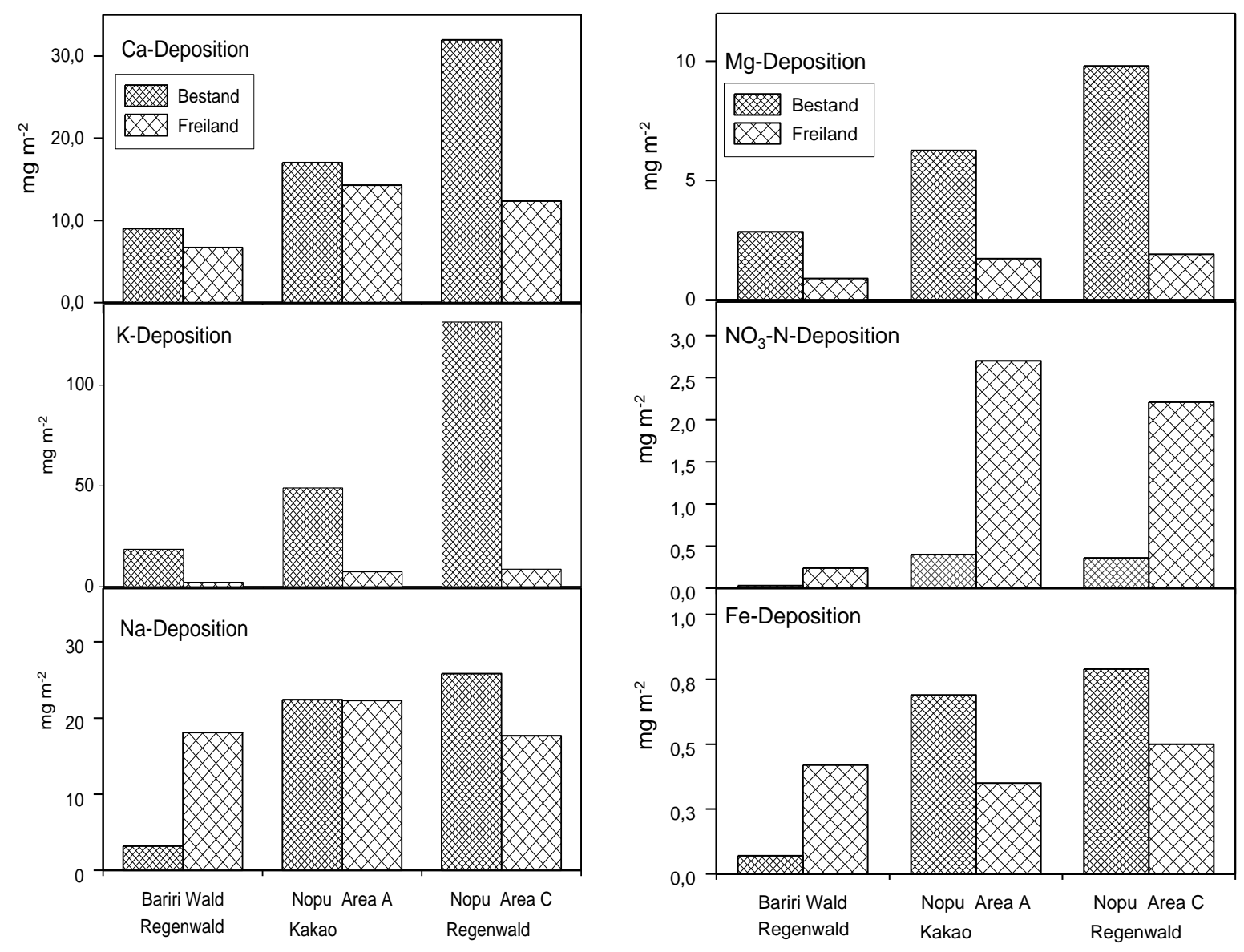

Abb. 5.7: Deposition an den verschieden Messstandorten im Bestandes- und Freilandniederschlag

Abb. 5.7: Deposition at different sites for throughfall and incident rainfall

Werden die Verhältnisse zwischen Bestandesdeposition und Freilanddeposition berechnet, zeigen sich ähnliche Abstufungen zwischen den Standorten, wie dies für die absoluten Frachten der Bestandesdeposition bereits beschrieben wurde. Die Elementverhältnisse zwischen Bestandesdeposition und Freilanddeposition sind am Standort Bariri am engsten und zeigen eine Abreicherung im Bestand für die Elemente $\mathrm{Al}, \mathrm{Fe}, \mathrm{Na}, \mathrm{NO}_{3}-\mathrm{N}$ und $\mathrm{P}$ (Tab. 5.5). Die Elemente $\mathrm{Ca}, \mathrm{K}, \mathrm{Mg}$, und $\mathrm{Mn}$ werden im Bestandesniederschlag um die Faktoren zwischen 1,3 für $\mathrm{Ca}$ und 8,2 für $\mathrm{K}$ angereichert. 
Tab. 5.5: Massenverhältnisse zwischen Bestandesniederschlag und Freilandniederschlag an den Standorten Bariri und Nopu

Tab. 5.5: Mass ratio between the throughfall and incident rainfall deposition at the sites in Bariri and Nopu

\begin{tabular}{ll|ccccccccc} 
& & Al & Ca & Fe & K & Mg & Mn & Na & NO3 & $P$ \\
\hline Bariri Wald & (Regenwald) & 0.1 & 1.3 & 0.2 & 8.2 & 3.2 & 3.1 & 0.2 & 0.1 & 0.5 \\
Nopu Plot A & (Kakao) & 0.7 & 1.4 & 2.0 & 6.6 & 3.6 & 2.1 & 1.0 & 0.1 & 3.0 \\
Nopu Plot C & (Regenwald) & 1.3 & 2.5 & 1.6 & 15.1 & 5.1 & 1.0 & 1.5 & 0.2 & 18.0
\end{tabular}

Eine Abreicherung der Elemente im Bestandesniederschlag wird an dem Standort Nopu Plot A für Al und $\mathrm{NO}_{3}-\mathrm{N}$, bzw. nur für $\mathrm{NO}_{3}-\mathrm{N}$ am Waldstandort Nopu Plot $\mathrm{C}$ beobachtet. Die Elemente $\mathrm{Ca}, \mathrm{Mg}$, $\mathrm{NO}_{3}-\mathrm{N}$ zeigen für Nopu Plot $\mathrm{A}$ ungefähr dieselben Faktoren wie sie für Bariri gefunden wurden, und übersteigen diese leicht für den Standort Nopu Plot C (Tab. 5.5), wenn auch die absoluten Mengen sich deutlich unterscheiden (Tab. 5.4). Stärker angereichert werden die Elemente Al, Fe und $\mathrm{Na}$, sowie P, welches um das 6-fache höher im Bestandesniederschlag Nopu Plot A gefunden wurde (Tab. 5.5). Unerwartet ist die geringere Anreicherung von $K$ in Nopu Plot A. Hier sei darauf hingewiesen, dass es sich um Anreicherungsfaktoren und nicht um absolute Mengen handelt. Die absoluten Mengen, in Tab. 5.4 gezeigt, sind im Freilandniederschlag Bariri deutlich geringer.

Die Faktoren der Elemente des Waldstandortes Nopu Plot $C$ steigen gegenüber der Kakaoplantage Nopu Plot A um das doppelte an, P steigt in der Bestandesdeposition sogar um den 6-fachen Wert gegenüber Nopu Plot A. Das Element Na zeigt nur einen leichten Anstieg, da es ein meerbürtiges inhärentes Element darstellt. Dagegen zeigen die Elemente Fe und $\mathrm{Mn}$ niedrigere Verhältnisse an (Tab. 5.5).

Folgender Trend lässt sich festhalten: die Element- und Nährstoffeinträge sind in Bariri in Freilandund Bestandeseintrag am geringsten und werden je nach Element $\mathrm{An}-(\mathrm{Ca}, \mathrm{K}, \mathrm{Mg}, \mathrm{Mn}$ ) oder Abgereichert ( $\mathrm{Al}, \mathrm{Fe}, \mathrm{Na}, \mathrm{P}$ ). Die Freilanddepositionen im tiefer gelegenen Nopu Open Area A sind für die meisten Elemente höher als im oberen, näher an der Brandrodungsfläche gelegenen Waldfläche Nopu Open Area C. Bei der Bestandesdeposition und den Elementverhältnissen verhält es sich umgekehrt. Im Waldstandort Nopu Plot $C$ sind die Depositionen und die Verhältnisse höher, und zwar um ein vielfaches, als die Depositionen im tiefer gelegenen Bereich Nopu Plot A.

Werden die Nährstoffeinträge aus anthropogenen Quellen ( $\mathrm{NO}_{3}-\mathrm{N}$ und $\mathrm{P}$ ) zeitlich abgetragen, zeigen sich an den Standorten Nopu für $\mathrm{NO}_{3}-\mathrm{N}$ Depositionsspitzen im Juni 2007 sowie Dezember 2007 und März 2008 (Abb. 5.8). Dies korreliert mit den trockeneren Monaten im Untersuchungszeitraum. Die Depositionsspitzen für $\mathrm{NO}_{3}-\mathrm{N}$ im Freilandniederschlag werden in den Monaten Januar 2008 und März 2008 erfasst, also etwas später als der Haupteintrag im Bestand. Die Skalierung der Achsen zeigt, dass es sich in der Bestandesdeposition um eine Abreicherung des $\mathrm{NO}_{3}-\mathrm{N}$-eintrages gegenüber der Freilanddeposition handelt. Die Höchsteinträge für $\mathrm{P}$ werden einen Monat später verzeichnet und zeigen keine Depositionsspitzen wie der $\mathrm{NO}_{3}$-N-Eintrag. Jedoch kommt es an den Standorten Nopu, wie bereits oben beschrieben, zu einem starken Anstieg um fast das 10- bis 20-fache im P-Eintrag. Die Depositionswerte für Bariri, sowohl im Bestand als auch im Freiland, liegen deutlich unter den 
Depositionen der Standorte in Nopu. Leichte Depositionserhöhungen im Freiland werden zeitgleich zu den Standorten Nopu Plot C und Nopu Plot A registriert.

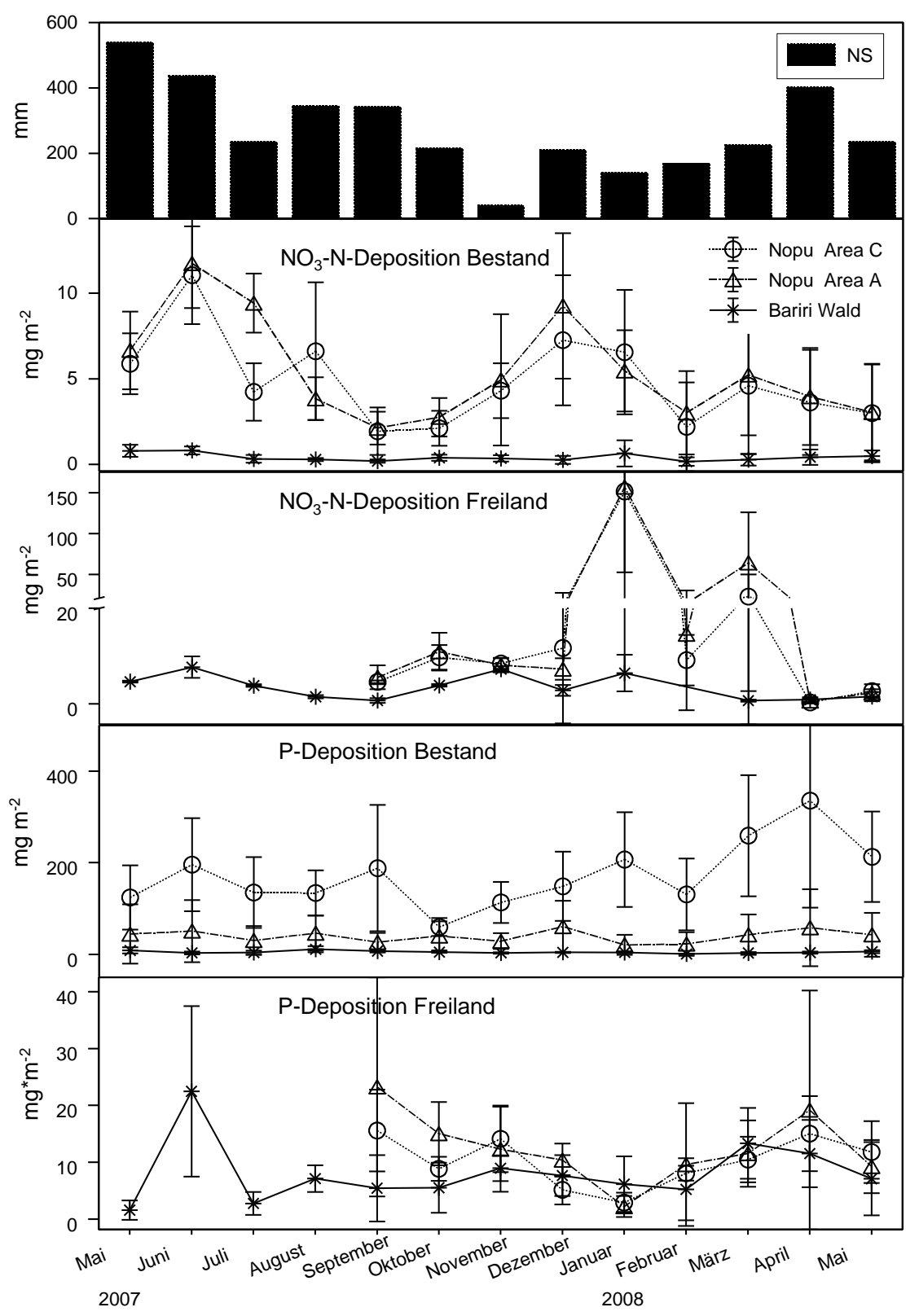

Abb. 5.8: Monatliche Niederschläge und Depositionseinträge während des Untersuchungszeitraumes an den Standorten Nopu und Bariri für $\mathrm{NO}_{3}-\mathrm{N}$ und $\mathrm{P}$

Fig. 5.8: Monthly precipitation and deposition of $\mathrm{NO}_{3}-\mathrm{N}$ und $\mathrm{P}$ during the experimental time at the sites Nopu and Bariri

\subsection{Diskussion}

Die gefundenen Frachtmengen in den Freiland- und Bestandesdepositionen der Standorte unterscheiden sich erheblich. Bariri zeigt mit den gefundenen Frachten im Bestand und der Freilanddepositionen geringe Werte, die bis zu 10-fach geringer sind als die Werte an den Standorten in Nopu. Dies wurde erwartet, da der Waldstandort Bariri abgelegen und rund 50 km Luftlinie von 
den Untersuchungsflächen in Nopu entfernt ist, keine Düngemittel in näherer Umgebung zum Einsatz kommen, Brandrodung in näherer Umgebung nicht stattgefunden hat (Abb. 5.2), der Bestand deutlich dichter ist und Nährstoffe via Staub im oberen Kronenraum bereits von saprophytisch lebenden Pflanzen wie Baummoose, Farne und Orchideen teilweise aufgenommen werden.

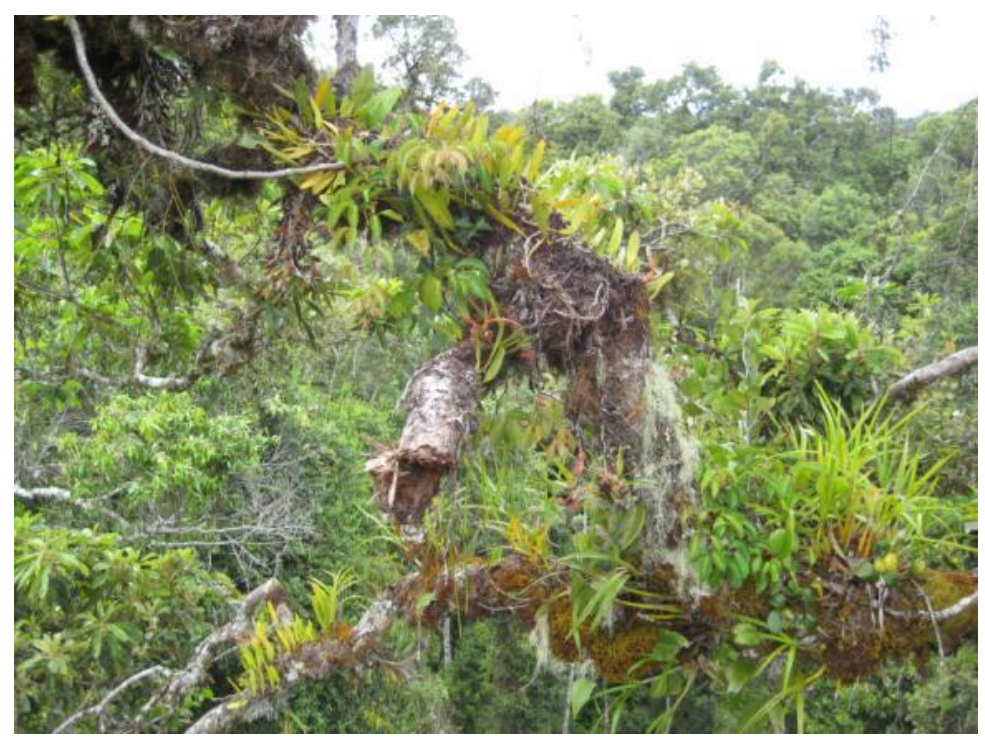

Abb. 5.9: Saprophytische Besiedlung im Kronenraum am Standort Bariri Wald (Bild: G.Gerold)

Fig. 5.9: Sapropyts at the forest site at Bariri Forest (picture: G.Gerold)

Dass in Bariri keine anthropogenen Schadstoffe eingetragen werden, zeigt die sehr geringe $\mathrm{NO}_{3}-\mathrm{N}$ Deposition von $0,2 \mathrm{~kg} \mathrm{ha}^{-1}$ (Tab. 5.3). Dieser Wert liegt weit unter den $0,8 \mathrm{~kg} \mathrm{ha}^{-1} \mathrm{a}^{-1}$, die als Frachten im kontinentalen Brasilien sowie den $1,5 \mathrm{~kg} \mathrm{ha}^{-1} \mathrm{a}^{-1}$ in küstennahen tropischen Regenwäldern gemessen wurden (Tab.5.1; Köhler et al., 2012b). Der Standort Bukit Koto Tabang in Sumatra/Indonesien wird von Ayers et al. (2002) als naturbelassener Referenzstandort (remoted area) angeführt mit einem $\mathrm{NO}_{3}-\mathrm{N}$-Depositionseintrag von $0,6 \mathrm{~kg} \mathrm{ha}^{-1} \mathrm{a}^{-1}$. Die weite Unterschreitung dieser Werte zeigt, dass in weiten Teilen Zentralsulawesis nur geringe anthropogene $\mathrm{NO}_{3}-\mathrm{N}-$ Depositionen mit Frachten von kleiner $0,8 \mathrm{~kg} \mathrm{ha}^{-1} \mathrm{a}^{-1}$ und besonders am Standort Bariri keine anthropogenen Einträge statt finden.

Alkali- und Erdalkalimetalle werden generell in Bestandesniederschlägen angereichert (Burghouts et al. 1998; McDowell et al. 1994; Hetzel 1999; Lanfer 2003; Fischer et al. 2008; Gutzler 2011). Durch den Protonenaustausch an der Blattoberfläche mit nachfolgender Auswaschung wird das Element $\mathrm{K}$ in hohem Umfange im Bestandsniederschlag angereichert (Tukey 1970; Parker 1983; Burghouts et al. 1998; Fischer 2004).

Mit einer Anreicherung von 1,3 für Ca gegenüber der Freilanddeposition liegt der Wert unterhalb des Wertes von 2,8, den Burghouts et al. (1998) fand. Andere Untersuchungen fanden Werte die mit 5,5 bis 7,2 deutlich über diesem Wert liegen (Tab. 5.6). Der Anreicherungsfaktor von K mit 8,2 steht einem Literaturwert zwischen 19 und 20 gegenüber (Tab. 5.6). Nur Fischer et al (2008) fand einen Faktor von 10,7, der dem Wert nahe kommt. Für Mg wurde ein Faktor ermittelt, der mit 3.2 etwas 
über dem Wert von McDowell (1994) mit 2,7 liegt. Für Na wurde eine Abreicherung ermittelt. Die berechneten Faktoren für den Waldstandort Bariri entsprechen bis auf Na den in der Literatur gefundenen Faktoren. In der vorliegenden Untersuchung wurden die Faktoren aus den akkumulierten Frachten bestimmt, während in den vergleichenden Untersuchungen eine Berechnung aus den Depositionen der direkten Niederschlagswerte oder aus gemittelten Niederschlägen (bulk deposition) vorgenommen wurde.

Die Anreicherungsfaktoren für die Alkali - und Erdalkalielemente am Standort Nopu Plot A (Tab. 5.6), der Kakaoplantage entsprechen den von Gutzler (2011) bestimmten Faktoren. Für die Faktoren im Regenwaldstandort Nopu Plot C wurden höhere Werte für die Anreicherung gefunden (Tab. 5.6), die sich im Bereich der Literaturwerte (Tab. 5.6; Burghouts et al. 1998, Fischer et al. 2008) befinden.

Tab. 5.6: Anreicherungsfaktoren für Elementdepositionen an Regenwaldstandorten und Kakaoplantagen (n. Gutzler 2011)

Tab. 5.6: Enrichmentfactors for Deposition in Rain forest sites and cacao plantagions

\begin{tabular}{l|cccc} 
& $\mathrm{Ca}$ & $\mathrm{K}$ & $\mathrm{Mg}$ & $\mathrm{Na}$ \\
\hline Anreicherung & & & & \\
Tropischer Regenwald & & & & \\
Literatur & $2,8-7,2$ & $10,7-19,3$ & $2,7-10,2$ & $0,9-2,3$ \\
Gutzler & 4,0 & 20,2 & 6,8 & 1,2 \\
Bariri Wald (diese Untersuchung) & 1,3 & 8,2 & 3,2 & 0,2 \\
Nopu Plot C (diese Untersuchung) & 2,5 & 15,1 & 5,1 & 1,5 \\
Anreicherung & & & & \\
Kakaoplantage & & & & \\
Literatur & $1,4-2,7$ & $4,8-17,4$ & $2,3-9,2$ & $0,7-1,0$ \\
Gutzler & 1,3 & 6,2 & 3,4 & 0,7 \\
Nopu Plot A (diese Untersuchung) & 1,4 & 6,6 & 3,6 & 1,0
\end{tabular}

Burghouts (1998), McDowell (1994), Hetzel (1999), Lanfer(2003), Fischer et al. (2008) und Gutzler(2011)

Unerwartet war eine Anreicherung der Nährstoffe $\mathrm{Fe}, \mathrm{Mn}$ und besonders $\mathrm{P}$ an den Standorten in Nopu und zusätzlich eine Anreicherung von Al am Regenwaldstandort Nopu Plot C. Die Tab. 5.5 zeigt eine mit zunehmender Nähe zum Brandrodungsgebiet zunehmende Depositionsfracht in den Beständen, die durch die Freilanddeposition nicht zu erklären ist. Auffällig ist die Anreicherung von $P$ und Al im Bestandesniederschlag am Standort Nopu Plot C.

Gutzler (2011), der über 24 Monate an den Standorten Nopu Depositions- und Bestandeseinträge erfasste, bestätigt weitestgehend die Anreicherungsfaktoren der Bestandeseinträge für die Regenwald- und Kakaoplantage in Nopu. Die von Gutzler (2011) berechneten Depositionsmengen werden im Mittel zwischen 2\% und 30\% unterschätzt. Stark unterschätzt wird der Eintrag von K (35\%). Unterschätzungen im Bereich von 100\% und mehr finden sich bei Gutzler (2011) im Eintrag von Al, $\mathrm{Mn}$ und $\mathrm{NO}_{3}-\mathrm{N}$. Er weist darauf hin, dass die Nährstoffe in wässriger Lösung analysiert und die 
Bestimmungskonzentrationen gerade für diese Elemente mehrfach unterschritten wurden. In der Arbeit von Gutzler (2011) werden auch die geringeren Freilanddepositionseinträge des Standortes Nopu Open Area bestätigt, die er für seine Berechnung der Anreicherungsfaktoren in den Bestandesflächen als Freilandbezug verwendet.

Abreicherungen im Bestandesniederschlag (Tab. 5.5), wie diese in Bariri für P-Depositionen in der vertikalen Bestandspassage beobachtet wurden, beruhen auf Filtereffekten bzw. Zwischenspeicherung und Aufnahme durch die Epiphyten (Tab. 11.5 in Giambelluca and Gerold (2011). Eine P-Aufnahme über Blätter ist bekannt und wird bei der Anwendung von P-haltigen Düngemittel zunutze gemacht.

Dieser Filtereffekt gilt auch für N-, Ca-, K- und Mg-Frachten, die zunächst im Kronenhumus (canopy humus, s. Nadkarni et al. 2004) zwischengespeichert werden. Während $\mathrm{Ca}, \mathrm{K}$ und $\mathrm{Mg}$ jedoch eine deutliche Anreicherung über den „wash-out“-Effekt im Kronenraum und zusätzlichem Blattaustausch (K) erfahren (Tab. 5.5), erfahren die Minimumelemente $P$ und $N$ eine Abreicherung im Bestandsniederschlag, der für $\mathrm{NO}_{3}-\mathrm{N}$ in Bariri mit dem Faktor 0,1 eine fast vollständige Aufnahme des mineralisierten Stickstoffes im Kronenraum zeigt.

Für $\mathrm{NO}_{3}-\mathrm{N}$ ist in Nopu sowohl bei Kakao wie im Regenwald eine Abreicherung gegeben, bei deutlich höheren absoluten Freiland- und Bestandsniederschlagsgehalten (Abb. 5.7). Für $\mathrm{P}$ wie auch für $\mathrm{Ca}, \mathrm{K}$ und $\mathrm{Mg}$ ist in Nopu im Regenwald eine etwa doppelt so hohe ( $\mathrm{Ca}, \mathrm{K}, \mathrm{Mg}$ ) und für $\mathrm{P}$ eine mehr als dreißigfache Anreicherung gegeben.

Während K pflanzenphysiologisch vor allem an Transport- und Steuermechanismen in der Pflanze beteiligt ist und mobil in der Pflanze bewegt wird, ist $P$ fest in die Erbanlagen und Enzyme eingebaut und stellt ein festes stationäres Element dar. Eine Blattauswaschung ist aus diesem Grund unwahrscheinlich und in der Literatur nicht belegt. Al ist für die meisten Pflanzen ein phytotoxisches Element und wird von der Pflanze weitestgehend nicht aufgenommen. Die höheren Frachten im Bestandesniederschlag gegenüber den geringeren Frachten im Freiland können keiner der angeführten Quellen zugewiesen werden. Die Quelle für die P- und Al-Anreicherung im Regenwaldstandort Nopu bleibt unbekannt. Durch die im Bestandesniederschlag gefundenen hohen Nährstoffgehalte gegenüber den geringeren Freilanddepositionen entstehen (theoretisch) Bilanzlücken, die durch Einträge aus weiteren Quellen geschlossen werden müssen. Dass es sich um eine weitere Stoffquelle handeln muss und nicht um eine Auswaschung aus Blättern, zeigen auch die Bestandesniederschläge in Bariri, in denen eine Anreicherung von P- und Al nicht stattfindet.

Während des Messzeitraumes erfolgten unterhalb von Plot $C$ in Nopu frische Brandrodungen (Abb. 5.3), so dass die anthropogen-biogenen Aerosole, Rußpartikel und Gasemissionen mit den tagsüber vorherrschenden Hangaufwinden (Kleinhans 2005) in den Regenwaldbestand eingetragen wurden.

Mit der Brandrodung entsteht aus dem noch feuchten Pflanzenmaterial Wasserdampf, welcher mit Nährstoffen in verschieden Formen angereichert ist. Der Wassersdampf steigt mittels Konvektion nach oben und transportiert auf diesem Wege Nährstoffe in die oberhalb gelegenen Bestände. Trifft er dabei auf Waldflächen, wie dies an der steilen Hangkante in Nopu der Fall ist, kann der 
Wasserdampf an den Blättern abkühlen und die feinen Wassertropfen können von der Vegetation ausgekämmt werden, ähnlich den Effekten, die in Nebelwälder beschrieben wurden. Wird hingegen trockene Vegetation in heißen Feuern verbrannt, können sich Ruß, Staub und Aerosole bilden und tragen zur Bestandsniederschlagsanreicherung mit Nährstoffen bei.

Die höheren Werte von $\mathrm{P}$ und $\mathrm{NO}_{3}-\mathrm{N}$ in Nopu Plot $\mathrm{C}$ gegenüber Bariri zeigen deutlich den anthropogenen Einfluss aus der Brandrodung (Abb. 5.8). Der fast zeitgleiche Eintrag von $\mathrm{NO}_{3}-\mathrm{N}$ im Wald- und Kakaoplot weisen auf eine gemeinsame nahe liegende Quelle hin. Ein weiterer Hinweis, dass Nitrat aus der Brandrodung stammt, ist der etwas spätere, aber um ein vielfach höher Eintrag von $\mathrm{NO}_{3}-\mathrm{N}$ in den Freilandsammlern Nopu Open Area A und Nopu Open Area C. Im Freiland sind an Terminen mit Brandrodungsaktivität deutliche Peaks zu erkennen, die eine Anreicherung der Luft mit Stickoxiden bilden und über die Regenereignisse entsprechend eingetragen werden. (Abb. 5.8).

Die Deposition von $\mathrm{P}$ findet über Staub-, Nebel- oder Partikeleintrag statt. Für Bariri liegen die Freiland-P-Einträge in der Größenordnung maritim beeinflusster naturnaher Regenwaldökosysteme (Tab. 5.1) mit 0,4-0,7 kg ha ${ }^{-1} \mathrm{a}^{-1}$. Während der Freilandniederschlag von Nopu Plot A und Nopu Open Area sogar leicht niedriger als bei Nopu Plot $C$ ist (Tab. 5.3), steigt der Eintrag am Kakaoplot (Nopu Plot A) und am Regenwald (Nopu Plot C) enorm an. Dies kann nur durch lokale Quellen mit der Brandrodung und einem biogenen Partikeltransport (Aerosole) erklärt werden. P-Depositionen werden vermutlich als Partikel transportiert und haben daher eine geringere Reichweite und werden direkt in nahegelegene Bestände eingetragen. Der zeitliche Verlauf des P-Eintrages zeigt, dass dieser erhöhte Eintrag noch 1-2 Monate nach der unmittelbaren Brandrodung wirksam ist (Abb.5.8, Brandrodung im Februar/März 2008). Dies hängt mit der fortgesetzten Freisetzung unvollständig verbrannter biogener Aschepartikel und Aerosole auf der Brandrodungsfläche zusammen.

Für das Element $\mathrm{Mn}$ wird eine andere Quelle vermutet. In Bariri wurden höhere Werte gegenüber Nopu gefunden. Vermutet werden Blütenpollen als Quelle, da die Blühleistung und damit die Pollenproduktion vom Mn im Wald und Kakao abhängig ist (Boy et al., 2008a). Die Kakaoplantage zeigt durch die Kauliflorie des Kakao eine permanente Blütenausbildung und besitzt ein hohes Pollenpotenzial, während am Standort Nopu C im Unterwuchs der Bambusbestand einen 40\%-igen Deckungsanteil aufweist (Gutzler 2011) und damit eine geringere Pollenleistung anzunehmen ist.

\subsection{Schlussfolgerung und Ausblick}

Anthropogene Eingriffe in Regenwäldern über die Brandrodung setzen Nährstoffe frei, die umliegende Waldstandorte beeinflussen und zu einem höheren Nährstoffeintrag führen. Trotz regional relativ geringer Distanzen zwischen dem anthropogen kaum beeinflussten Regenwald von Bariri und dem durch fortschreitende kleinparzellige Brandrodung gekennzeichneten Palolotal mit Nopu zeigen die Freiland- und Nährstoffdepositionen von Bariri ein typisches Hintergrundsignal, ähnlich der anthropogen kaum beeinflussten Regenwaldbestände maritimer Großregionen. Die Abreicherung von $\mathrm{N}$ und $\mathrm{P}$ in Bariri weist indirekt auf die große Bedeutung der epiphytischen Kronenbestandteile hin, während die Bestandsabreicherung von Na bisher nicht bekannt war und weiterer Untersuchungen zum Verbleib bedarf. Für die Hauptkationen werden die aus der Literatur 
5 Depositionsmessung im Bestandesniederschlag

bekannten Anreicherungsfaktoren für Kakao und Regenwald bestätigt, wobei die deutlich höheren Faktoren in Nopu gegenüber Bariri mit aus der bereits anthropogen verursachten höheren Freilanddeposition resultieren. 
6 Überprüfung der Forschungshypothesen

6 Überprüfung der Forschungshypothesen 


\subsection{Laborbewertung der Sammler}

Die Entwicklung eines Probensammlers bedarf immer einer Vielzahl an Experimenten zur Überprüfung der Funktionsweise des Messgerätes sowie ein Vergleich mit bereits bestehenden Systemen zur Einschätzung der Messgeräte.

Mit der Bestimmung der Ladungskapazität des lonentauschers von $0,5 \mathrm{mmol}_{c} \mathrm{~g}^{-1}$ wurde die Grundlage gelegt, um Abschätzungen der benötigten Menge an lonentauscher im Feld zu berechnen. Nach Nicklas (2006) wurde eine Depositionsfracht im Freiland des Untersuchungsgebietes zwischen 8 $\mathrm{mmol}_{\mathrm{c}} \mathrm{g}^{-1}$ und $15 \mathrm{mmol}_{\mathrm{c}} \mathrm{g}^{-1}$ ermittelt. Um ausreichend Pufferkapazitäten im Sammler bereit zu stellen, wurde die berechnete Menge von $25 \mathrm{~g}$ lonentauschermaterial verdoppelt und eine rechnerische Belegung zwischen $32 \%$ und $60 \%$ erreicht.

Die Überprüfung der IER-Passiv-Sammler wurde unter verschieden Aspekten durchgeführt. Der Sammler sollte vollständig die lonenfracht von sowohl hoch konzentrierten als auch gering konzentrierten Mono- und Multielementlösungen entziehen. Der Entzug und die Festlegung von Ionen am lonentauscher ist elementabhängig und wird von der Ladung und dem lonendurchmesser bestimmt. Elemententzüge zwischen $79 \%$ und $98 \%$ (Tab. 2.3) über alle Konzentrationsbereiche spiegeln einen ausreichenden Entzug von Ionen wider, der auch in der Literatur bestätigt wird (Crabtree and Trudgill, 1981; Simkin et al., 2004).

H1) Die entwickelten Passivsammler können perkolierenden Lösungen Nährstoffe hinreichend entziehen und am lonentauscher festlegen. Dadurch ist eine Nährstofferfassung im Niederschlagswasser möglich.

Es konnte gezeigt werden, dass perkolierendes Regenwasser nahezu vollständig entsalzt werden kann. Die Hypothese H1 wurde somit unterstützt.

Der zweite Schritt zur Bestimmung der Depositionsfrachten ist die Remobilisierung bzw. Extraktion der lonen von den Ionentauscheroberflächen. Die Extraktion ist eine Gleichgewichtseinstellung der Lösungsphase mit dem Extraktionsmittel und den Ladungsträgern am lonentauscher. In kurzen Extraktionsschritten ist eine vollständige Extraktion nicht zu erwarten. Ähnlich den Prozessen bei der Festlegung von lonen werden diese je nach Ladung und Bindungsstärke unterschiedlich leicht von Ionentauscher remobilisiert. Die Wiederfindungsrate der Elemente aus dem Extrakt lag mit $47 \%$ für $\mathrm{Ca}$ als unterster Wert und 92\% für $\mathrm{Na}$ (Tab. 2.4) deutlich geringer als die Festlegungsrate am Ionentauscher (Tab. 2.3). Einzig $\mathrm{NO}_{3}-\mathrm{N}$ wurde mit $105 \%$ vollständig remobilisiert. Die höhere Wiederfindung wurde auf Kontamination aus dem Extraktionsmittel und dem Ionentauscher zurückgeführt, wobei höhere Wiederfindungen bereits durch Crabbtree und Trugil (1981) beschrieben wurden. Durch mehrmalige Extraktion konnte die Wiederfindung der anderen lonen erhöht werden. Die Massendifferenz zwischen Festlegung und Remobilisierung muss auf dem 
Ionentauscher verblieben sein und kann deshalb mit einem Korrekturfaktor hochgerechnet werden (Tab. 2.4).

H2) An den lonentauschern festgelegte Nährstoffe können durch Extraktion quantitativ zurückgetauscht werden. Blindwerte können zur Korrektur der Hintergrundwerte genutzt werden.

Hypothese 2 kann nur teilweise gestützt werden. Wie bereits dargestellt, ist ein quantitativer Rücktausch nicht vollständig möglich. Anhand eines Korrekturfaktors kann jedoch der extrahierte Depositionsanteil elementspezifisch auf die gesamtfixierte Frachtmenge berechnet werden.

Verluste von Depositionsfrachten können durch eine Remobilisierung und Verlagerung im Ionentauscher während der Sammelphase im Geländeeinsatz entstehen (Chromatographieeffekt). Eine Überprüfung zeigte, das Depositionsfrachten vorwiegend in den oberen $6 \mathrm{~cm}$ stattfindeen und ein Durchbruch aufgrund der hohen Ladungsreserven im lonentauscher nicht zu erwarten ist.

H3) Es besteht ein Zusammenhang zwischen dem Aufbau, der Fließgeschwindigkeit im Sammler und der Effektivität in der Fixierung von lonen.

Der Aufbau des Sammlers trägt zur Fixierleistung bei. Ausschlaggebend ist die Kontakt- und Verweilzeit im lonentauschermaterial, um gelöste Ionen an den lonentauscher zu binden. Wird durch einen geknickten Aufbau die Fließgeschwindigkeit reduziert, erhöht sich, durch die längere Verweilzeit, die Fixierleistung des Sammlers. Im durchgeführten Versuch konnte so eine Steigerung der Fixierleistung von 3\% erreicht werden (Tab. 2.6). Durch die geringere Perkolationsleistung der geknickten Sammler kann es bei hohen Wassermengen allerdings zu einem Überlaufen des Sammlers kommen. Die Hypothese 3 wurde unterstützt. Allerdings steht der potentiellen $2 \%$ kalkulierbaren höheren Fixierleistung ein möglicher unkontrollierter Verlust an Regenwasser gegenüber. Deshalb wurden im Feld gesteckte Sammler eingesetzt.

Konzentrationen im Niederschlagswasser können sehr unterschiedlich sein und sind meist bei einsetzendem Regen am höchsten. Im Versuch mit sich verdünnenden Lösungen wurde die Beobachtung, dass eine vorwiegende Fixierung der Frachten in den ersten $6 \mathrm{~cm}$ des Sammlers stattfindet, bestätigt. Im Vergleich wurden Lösungen mit sinkender Konzentration elementspezifisch zwischen $1 \%$ und $23 \%$ besser fixiert als perkolierende Lösungen mit konstanter Konzentration (Tab. 2.7).

Die angenommenen Hypothesen werden weitestgehend unterstützt. Die Sammler besitzen gute Sammeleigenschaften, um anfallende Depositionsfrachten im Feld zu erfassen. Die Leistung des Sammlers steigt, je langsamer anfallendes Regenwasser den Sammler durchströmt und je geringer die Konzentration der Depositionsfracht im Niederschlagswasser ist. In beiden Fällen wird eine höhere Verweilzeit und damit eine höhere Kontaktmöglichkeit mit den freien Ladungsträgern ermöglicht. 


\subsection{Freilandbewertung der Sammler}

Depositionsfrachten im Freiland sind unbekannt und eine Überprüfung der Sammler im Feld ist nur durch den Vergleich zweier Messsysteme möglich. Der Vergleich der IER-Sammler gegenüber klassischen Bulkdepositionssammlern ergab, dass Passivsammler meist zu leicht höheren Ergebnissen neigen. Die Elemente $\mathrm{Ca}, \mathrm{Mg}$, $\mathrm{Na}$, Fe und P lagen im Vergleich zwischen 103\% und 134\% höher als die Frachten, die in den Bulkdepositionssammlern gefunden wurden. Deutlich höhere Werte der eingetragenen Frachten wurden für die Stoffe $\mathrm{NO}_{3}-\mathrm{N}$ (434\%) und $\mathrm{Al}$ (484\%) mit den IER-Sammlern gemessen. Niedrigere Frachten wurden für $\mathrm{Mn}(81 \%)$ und K (65\%) erfasst.

H4) Die Erfassung der Bulk-Niederschlagsdeposition im Freiland zeigt keine Unterschiede zwischen den IER-Passivsammlern und konventionellen Niederschlagssammlern

Die Hypothese 4 wurde für die Elemente $\mathrm{Ca}, \mathrm{Mg}, \mathrm{Na}$, Fe und $\mathrm{Mn}$ weitestgehend gestützt, da die Depositionsfrachten in beiden Sammelsystemen vergleichbar sind (Tab. 3.2) trotz signifikanter Unterschiede, die meist nicht relevant waren. Abzulehnen ist die Hypothese für die Elemente Al und $\mathrm{NO}_{3}-\mathrm{N}$, die deutlich höhere Frachten in den Passivsammlern zeigen. Die höhere Schätzung der erfassten Depositionsfrachten wird auf die integrative Sammeleigenschaft des IER-Passivsammlers zurückgeführt, die auch kleine Mengen und Staubdepositionseinträge erfasst. Durch die Extraktion des Ionentauschermaterials können, gegenüber dem Niederschlagswasser, deutlich höhere Konzentrationen im Extrakt erreicht werden. Somit werden die IER Werte als realitätsnäher eingestuft.

H5) Durch das integrierende Sammelverfahren der IER-Sammler, verbunden mit der Gewinnung von Extrakten aus den lonentauschern, können höhere Konzentrationen in den Lösungen erreicht werden. Die Erfassung der Konzentrationen wird erleichtert und geringe Eintragsmengen können gemessen werden.

Die Hypothese 5 wurde unterstützt. Für die IER-Sammler wurden für alle Elemente in über $90 \%$ der Proben Konzentrationen gefunden, die über $0,1 \mathrm{mg} \mathrm{l}^{-1}$ (Tab. 3.4) liegen. Eine Ausnahme stellt das Element $\mathrm{Mn}$ dar, welches nur in knapp 16\% aller Proben über dieser Konzentration liegt. Ursache ist die sehr geringe Depositionsfracht von $0,1 \mathrm{mg} \mathrm{ha}^{-1} \mathrm{a}^{-1}$.

Die Bulkdepositionssammler, welche direkt Proben aus dem Regenwasser verwenden, liegen für Makroelemente wie $\mathrm{Ca}, \mathrm{K}$ und $\mathrm{Na}$ ebenfalls mit $90 \%$ aller Proben über dieser Konzentration. Elemente mit geringer Depositionsfracht wie $\mathrm{Al}, \mathrm{Fe}, \mathrm{Mg} \mathrm{Mn}$, und $\mathrm{P}$ liegen mit mehr als $85 \%$ aller Proben, für $\mathrm{NO}_{3}-\mathrm{N}$ sogar mehr als $97 \%$, unter diesem Konzentrationsbereich (Tab. 3.4). Diese 
Unterschiede zeigen deutlich das unterschiedliche Sammelverhalten der Messsysteme. Deutlich wird der Sammelunterschied auch in der Depositionsdifferenz zwischen beiden Systemen in Verbindung mit der Niederschlagsmenge, die mit $R^{2}=-0,81$ für Ca stark korreliert. Die Korrelation wird auf die Verbindung der Depositionsfrachten im Bulkdepositionssammler mit den Niederschlägen zurückgeführt, die einerseits in der Berechnungsgrundlage enthalten sind und bei zunehmender Niederschlagsmenge verdünnt werden. Da IER-Kollektoren wasserfrei messen, spielt die Regenmenge weder in der Erfassung noch zur Berechnung eine Rolle. Der Passivsammler besitzt große Vorteile besonders in der Erfassung geringer Depositionsfrachten wie Mangan, die mit $100 \mathrm{~g}$ ha ${ }^{-1} \mathrm{a}^{-1}$ im Bulkdepositionssammler nur schwer zu erfassen sind

Die Untersuchungen haben gezeigt, dass IER-Kollektoren für den Feldeinsatz zur Depositionsmessung geeignet sind. Die Messgeräte sind sowohl bei Starkregenereignissen als auch bei geringen Niederschlagsmengen einsetzbar. Durch die integrative Akkumulation der Nährstoffe können selbst geringe Depositionsfrachten bestimmt werden. Nachteilig ist die geringe zeitliche Auflösung der IERKollektoren. Einsatzspezifisch ist die Bestimmung von Blind- und Korrekturwerten durchzuführen.

\subsection{Heterogenität der Depositionserfassung im Freiland}

Verglichen mit anderen tropischen Regenwäldern weltweit wurden in Zentral Sulawesi geringe Depositionsfrachten erfasst. Der jährliche Eintrag an Nährstoffen im Untersuchungsgebiet des Lore Lindu National Parks lag für die Summe der Makroelemente in 2008 ( $\mathrm{Ca}, \mathrm{Mg}, \mathrm{K}, \mathrm{Na}$ ) zwischen $28 \mathrm{~kg}$ $\mathrm{ha}^{-1} \mathrm{a}^{-1}$ und $42 \mathrm{~kg} \mathrm{ha}^{-1} \mathrm{a}^{-1}$ (Tab. 4.1) und ist vergleichbar mit den Einträgen eines tiefer gelegenen Bergregenwaldes mit $11 \mathrm{~kg} \mathrm{ha}^{-1} \mathrm{a}^{-1}$ bis $37 \mathrm{~kg} \mathrm{ha}^{-1} \mathrm{a}^{-1}$ (Tab. 4.3) in Jamaika (Hafkenscheid, 2002).

Als anteilig hoch einzuschätzen sind die marinen $\mathrm{Na}$-Frachten, die als Salzpartikel von dem rund 50$150 \mathrm{~km}$ entfernt liegenden Ozean eingetragen werden. Der Anteil an der Gesamtdeposition lag für 2008 mit Depositionsfrachten zwischen $13 \mathrm{~kg} \mathrm{ha}^{-1} \mathrm{a}^{-1}$ und $24 \mathrm{~kg} \mathrm{ha}^{-1} \mathrm{a}^{-1,}$ zwischen 50\% bis 70\% (Tab. 4.1) und ist von klimaprägenden ozeanischen Luftmassen beeinflusst. Die Depositionsfracht liegt im unteren Bereich küstennaher tropischer Regenwälder (Tab. 4.3; Oziegbe et al, 2011; Hafkenscheid, 2002), jedoch über den geringeren Depositionsfrachten kontinentaler tropischer Wälder mit Einträgen zwischen 2,5 kg ha ${ }^{-1} a^{-1}$ und 3,5 kg ha ${ }^{-1} a^{-1}$ (Tab. 4.3; Filiso et al., 1999; Germer et al, 2007).

H6) Natürliche Stoffeinträge aus geogenen und marinen Quellen zeigen geringe zeitliche und räumliche Schwankungen, die auf den Abständen zu den Quellen beruhen. Große Unterschiede in den Stoffeinträgen weisen auf den Eintrag anthropogener Stoffe hin.

Die Hypothese 6 kann damit nur teilweise untermauert werden. An den Standorten zeigten sich große Schwankungen zwischen den eingetragenen Elementen auch aus natürlichen Quellen. Deutlich wird dies an den großen Unterschieden im Jahr 2008 für das Element $\mathrm{Na}$, welche in Unterschieden 
bis zu $10 \mathrm{~kg} \mathrm{ha}^{-1} \mathrm{a}^{-1}$ Standortspezifisch deponiert wurden und nicht aus anthropogenen Quellen stammt (Tab. 4.1).

Geogene Elemente wie $\mathrm{Fe}, \mathrm{Al}$, und $\mathrm{Mn}$ werden mit geringer Variabilität eingetragen, und aufgrund der engen Beziehung dieser Elemente in der PCA-Analyse wird eine gemeinsame Quelle angenommen (Abb. 4.8). Als Quelle werden trockene Staubdepositionen vermutet, die sich aus der Bewirtschaftung landwirtschaftlicher Nutzflächen ergibt und in diesen Landnutzungssystemen höhere Frachten zeigen. Anthropogene Einflüsse aus Industrieanlagen oder Brandrodungsflächen sind auszuschließen, da diese Landnutzungssysteme vergleichsweise geringe Depositionsfrachten an $\mathrm{NO}_{3}-\mathrm{N}$ zeigen (Tab. 4.2). Einen Anstieg der deponierten Frachten in den unterschiedlichen Landnutzungssystemen wurde für die Elemente $\mathrm{Ca}, \mathrm{K}$, und besonders für $\mathrm{NO}_{3}-\mathrm{N}$ beobachtet und der anthropogene Einfluss nachgewiesen.

Am naturnahen Waldstandort Bariri wurde für das geogene Element Ca ein Depositionseintrag von 4,6 $\mathrm{kg} \mathrm{ha}^{-1} \mathrm{a}^{-1}$ gemessen, der auf das Doppelte in landwirtschaftlichen Nutzflächen anstieg und mit $12,5 \mathrm{~kg} \mathrm{ha}^{-1} \mathrm{a}^{-1}$ in den Brandrodungsgebieten einen fast dreifach höheren Wert zeigt. $\mathrm{K}$, ein Element, das sowohl aus biogenen als auch marinen Quellen stammen kann, zeigt in den Depositionsfrachten einen ähnlichen Trend. Das aus anthropogenen Quellen stammende $\mathrm{NO}_{3}-\mathrm{N}$, welches in Bariri mit 0,2 $\mathrm{kg} \mathrm{ha}^{-1} \mathrm{a}^{-1}$ sehr gering eingetragen wird, steigt in den Brandrodungsflächen auf das 10-fache des Wertes mit $2 \mathrm{~kg} \mathrm{ha}^{-1} \mathrm{a}^{-1}$ an (Tab. 4.2). Die Steigerung der Depositionsfrachten in den unterschiedlichen Nutzungssystemen und die Lagen in der PCA-Analyse zeigen einen anthropogenen Einfluss deutlich, wobei typischerweise mit zunehmendem Abstand zu den Quellen (Brandrodungsflächen in Nopu) die Streuung der Einträge sinkt (Abb. 4.9). Die sinkende Eintragsintensität mit steigendem Abstand zur Quelle (Abb. 4.7) zeigt ein deutliches anthropogenes Signal.

H7) Anthropogene Schadstoffe werden auch in den Nationalpark eingetragen und deponiert.

Die Hypothese 7 kann für die Randbereiche des National Parks unterstützt werden. Flächen, die nahe der Brandrodungszone liegen, werden mit höheren Depositionsfrachten anthropogener Herkunft belastet als entfernt gelegene Fläche. Bei Flächen in ausreichend weitem Abstand sinkt die Deposition auf ein geringes Niveau ab. Die $\mathrm{NO}_{3}-\mathrm{N}$-Deposition beträgt in Bariri 0,2 $\mathrm{kg} \mathrm{ha}^{-1} \mathrm{a}^{-1}$ und liegt damit unter den Frachten, die Ayers (2002) für den naturbelassenen Regenwald Bukit Koto Tabang in Sumatra angibt. Abzuleiten ist, dass die Emission aus Brandrodungsflächen, zumindest bei kleineren nicht so heißen Feuern, regional begrenzt ist.

Keine Unterschiede zeigen sich in der P-Deposition gegenüber den Brandrodungsflächen und landwirtschaftlichen Nutzflächen (1,3 kg ha $\left.{ }^{-1} \mathrm{a}^{-1}\right)$. Für P werden als Quellen, nach Rollenbeck (2010) vorwiegend natürliche Ressourcen angegeben, während Mahowald (2005) die P-Deposition mit Biomasseverbrennung in Verbindung bringt. 
Bei den Einträgen von $\mathrm{P}$ zeigt sich im Unterschied zu NO $\mathrm{NO}_{3}-\mathrm{N}$ keine deutliche Eintragsspitze. In der Zeit der Brandrodung im November 2007 bis Februar 2008 und im März bis Mai 2008 zeigt sich ein leichter Anstieg an den Standorten Nopu. In der Zeit der Brandrodung werden verstärkt P-Emissionen freigesetzt, und eine Kopplung von P-Emissionen mit der Biomasseverbrennung erklärt die höheren Depositionsfrachten an den Brandrodungsstandorten. Die hohe P-Deposition in den landwirtschaftlichen Nutzflächen könnte durch die regelmäßige Verbrennung von Reisstroh auf den Feldern verursacht werden. Hinweise geben die Vielzahl der Feuer in der Feuerkarte Abb. 4.2 im Gebiet von Wanga, in dem überwiegend landwirtschaftlichen Nutzflächen zu finden sind. Auf diese Erntefeuer werden auch die leicht höheren $\mathrm{NO}_{3}-\mathrm{N}$ Einträge in den landwirtschaftlichen Nutzflächen zurück geführt.

H8) Emissionen aus Brandrodung sind die größte Quelle für anthropogene Nährstoffdepositionen in Sulawesi. Einzelereignisse verändern die Qualität der Depositionszusammensetzung und bestimmen die Jahresdeposition.

Die hohen Depositionsfrachten nahe der Brandrodungsflächen weisen deutlich auf erhöhte Emissionen von $\mathrm{NO}_{3}-\mathrm{N}, \mathrm{K}, \mathrm{Ca}$ und $\mathrm{P}$ aus Biomasseverbrennung hin. Durch das Fehlen dieser Depositionsfrachten am naturbelassenen Standort Bariri kann geschlossen werden, dass Depositionsfrachten aus anthropogen industriellen Emittenten (Ferntransport) in Zental Sulawesi keine Bedeutung besitzen. Daher kann Hypothese 8 angenommen werden.

\subsection{Bestandesmessungen}

Die an den Standorten Bariri Wald (tropischer Regenwald), Nopu Plot A (Kakao-Plantage) sowie Nopu Plot C (tropischer Regenwald) erfassten Bestandesdepositionen zeigten erhebliche Unterschiede in den Frachtmengen. Depositionsmengen am Standort Bariri Wald waren um das 2- bis 11-fache geringer, die P-Deposition sogar um das 40-fache geringer als die in den Nopu Standorten erfassten Elementfrachten (Tab. 4.4). Einzig das Element $\mathrm{Mn}$ wurde in Bariri mit der höchsten Fracht gemessen. Dass Bariri als naturbelassener Standort mit geringen anthropogenen Frachten angesehen werden kann, zeigen die geringen $\mathrm{NO}_{3} \mathrm{~N}$-Frachten. Mit einem Jahreseintrag von 0,2 -0,4 kg ha- $\mathrm{a} \mathrm{a}^{-1}$ (Tab. 3.1) liegt Bariri unterhalb der für Bukit Koto Tabang angegebenen Frachtmenge $\left(0,6 \mathrm{~kg} \mathrm{ha}^{-1} \mathrm{a}^{-1}\right)$, einem naturbelassenen Referenzstandort für die Untersuchung von Ayers et al. (2002) in Sumatra/Indonesien oder den $0,8 \mathrm{~kg} \mathrm{ha}^{-1} \mathrm{a}^{-1}$ die als untere Fracht in lateinamerikanischen Regenwaldstandorten angegeben wird (Tab. 3.3). Die erhöhten $\mathrm{NO}_{3}-\mathrm{N}-$ Frachten in Nopu werden ausschließlich auf Emissionen aus den nahegelegenen Brandrodungsflächen zurückgeführt.

Ebenfalls auf die Emissionen aus Brandrodungsflächen werden die hohen P-Depositionen in den Bestandesmessungen in Nopu zurückgeführt. In Bariri wird P mit $0,7 \mathrm{~kg} \mathrm{ha}^{-1} \mathrm{a}^{-1}$ in vergleichbarer Größenordnung anderer tropischer Regenwälder eingetragen (Tab. 5.4). Im Bestand in Bariri Wald erfolgt eine Abreicherung, die auf die saprophytische Besiedelung des Kronenraumes zurückgeführt wird (Abb. 5.9). 
Mit rund $1 \mathrm{~kg} \mathrm{ha}^{-1} \mathrm{a}^{-1}$ liegt der P-Eintrag im Freiland an den Standorten in Nopu leicht über diesem Wert. In den Beständen in Nopu werden allerdings Frachten von 3-8 $\mathrm{kg} \mathrm{ha}^{-1} \mathrm{a}^{-1}$ erfasst. Dies entspricht Werten die 10- bis 40-fach höhere sind als in Bariri. Besonders am Standort Nopu Plot C, dem Regenwaldstandort, kommt es zu einer 18-fachen Anreicherung von $\mathrm{P}$ im Bestandesniederschlag.

H9) Brandrodung bedingt eine erhebliche Elementanreicherung im Bestandsniederschlag.

Durch die höheren Einträge, besonders aus der anthropogen verursachten Brandrodung, wird die Hypothese 9 unterstützt. Dass die Quantität damit in den Beständen sich verändert, wird aus den Frachtmengen in Tab 5.4 ersichtlich.

H10) Die Quantität der Bestandesdeposition wird durch umgebende Landnutzungssysteme bestimmt.

Die oben ausgeführte Bedeutung der P-Anreicherung belegt deutlich den Einfluss der Landnutzung und unterstützt daher Hypothese 10. In der Literatur wird ein Auswaschen von P aus Blättern nicht beschrieben. Die Anreicherung muss auf einen weiteren externen Eintrag in Form von Nebel oder Partikeln aus Brandrodungsereignissen zurück geführt werden. Der Anstieg wurde in Bariri nicht beobachtet.

Den Einfluss der Landnutzungssysteme auf Depositionsfrachten zeigt auch Tab. 4.2 mit unterschiedlichen Frachtmengen in den unterschiedlichen Nutzungssystemen. Für die Elemente $\mathrm{Ca}$, $\mathrm{K}, \mathrm{Mg}$ und $\mathrm{NO}_{3}-\mathrm{N}$ wurde eine Zunahme in den Depositionswerten von naturbelassenem Regenwald (Bariri) zu den Brandrodungsflächen festgestellt. Wie ober bereits angeführt, ist ein deutlicher Anstieg für die Ca-Deposition zu verzeichnen (Hypothese 9). Noch deutlicher tritt dies bei den Einträgen von $\mathrm{NO}_{3}-\mathrm{N}$ mit $0,2 \mathrm{~kg} \mathrm{ha}^{-1} \mathrm{a}^{-1}$ im naturbelassenen Regenwald auf, der sich in den landwirtschaftlichen verdoppelt und in den Brandrodungsflächen verzehnfacht.

\subsection{Ausblick}

In der vorliegenden Arbeit wurde die Entwicklung und Kalibrierung eines Passivsammlers auf Ionentauscherbasis vorgestellt. Die zufriedenstellende Anwendung ermöglichte eine Depositionserfassung im Lore Lindu Nationalpark in einer Fläche von 2310 km². Damit konnte nach vorliegendem Wissens erstmalig in den Tropen zur Erfassung von Bulkdepositionen verbunden mit Bestandesdepositionen auf der Mesoskala gemessen werden. Mit IER-Kollektoren ist es möglich, einen Beitrag zur Erfassung unterschiedlicher Nährstoffkreisläufe und dem Transfer zwischen Ökosystemen zu leisten und auf einer weiter angelegten Skala zu erfassen. Durch die einfache Probenbehandlung können lokale Hilfskräfte in den Messbetrieb einbezogen werden.

Die Möglichkeiten gelten insbesondere für Mikronährstoffe wie Mn oder auch Schwermetalle, deren Depositionsfrachten im Regenwasser zur Erfassung mit herkömmlichen Methoden oft zu gering ist. Durch die Erhöhung der Konzentration im Extrakt können geringe Depositionsmengen erfasst und bewertet werden. Depositionsfrachten von Mikronährelementen können mit Auswirkungen im 
6 Überprüfung der Forschungshypothesen

Ökosystem wie Beispielsweise Blühinduktion in Verbindung gebracht werden. Damit eröffnet sich ein Feld der Prozessforschung, in dem Steuergrößen gemessen und in einem komplexen System erklärt werden können. 


\section{Literaturverzeichnis}

Aas, W., Shao, M., Jin, L. L., T, Z., D, X. R., \& al, e. (2007). Air concentrations and wet deposition of major inorganic ions at five non-urban sites in China 2001-2003. Atmospheric Environment , S. 17061716.

Adams, M. B. (2003). Ecological issues related to $\mathrm{N}$ deposition to natural ecosystems: research needs. Environ. Int. , 29, S. 189-199.

Aldrian, E., \& Susanto, R. (2003). Identification of three dominant rainfall regions within Indonesia and their relationship to sea surface temperature. 'International Journal of Climatology , 23, S. 14351452.

Andreae, M. O., \& Merlet, P. (2001). Emission of trace gases and aerosols from biomass burning. Global Biogechemical Cycles, 15 (4), S. 955-966.

Andreae, M. O., Talbot, R. W., Berresheim, H., \& Beecher, K. M. (1990). Precipitation chemistry in Central Amazonia. Journal of Geophysical Research (95), S. 16987-16999.

Ashagrie, Y., \& Zech, W. (2010). Water and nutrient inputs in rainfall into natural and managaged forest ecosystems in south-eastern highlands of Ethiopia. Ecology and Hydrologie, 10, S. 169-182.

Ayers, G. P., Peng, L. C., Gillestt, R. W., \& Fook, L. S. (2002). Rainwater composition and acidity at five sites in Malaysia, in 1996. Water Air and Soil Pollution , 133, 15-30.

Berlage, H. P. (1949). Rainfall in Indonesia. Mean rainfall figures for 4339 rainfall stations in Indonesia 1879-1941. (E. e. Department van Verkeer, Hrsg.)

Boy, J., \& Wilke, W. (2008b). Tropical Andean forest derives calcium and magnesium from Saharan dust. Global Biogeochemical Cycles, 22, S. GB1027.

Boy, J., Rollenbeck, R., Valarezo, C., \& Wilke, W. (2008a). Amazonian biomass burning-derived acid and nutrient deposition in the north Andean montane forest of Ecuador.Biogeochem Cycles 22, S. GB4011 doi:10.1029/2007GB003158..

Bruijnzeel, L. A. (1991). Nutrient input-output budgets of tropical forest ecosystems: a review. Journal of Tropical Ecology (7), S. 1-24.

Burghouts, T., van Straalen, N., \& Bruijnzeel, L. (1998). Spatial heterogeneity of element and litter turnover in a Bornean rain forest. Journal of Tropical Ecology (14), S. 477-506.

Bytnerowicz, A., Schrumpf, M., Zech, W., Axmacher, J. C., Lyaruu, H. V., Alonso, R., et al. (2002). . (2002): Summer-time distribution of air pollutants in Sequoia National Park, California. Environmental Pollution, 118, S. 187-203.

Calil, F., Schumacher, M., witschorek, R., Lopes, V., Viera, M., \& Liberalesso, E. (2010). Ion input via rainwater in the southern region of rio grandedo sul,Brazil. Lavras, 16, S. 373-380.

Chang, J. S., Isaksen, I., Madronich, S., Stockwell, W., Walcek, R., \& al, e. (1987). A three-dimensional Eulerian acid deposition model: Physical concepts and formulation. Journal of Geophysical Research (92), S. 14681-14700. 
Chunyong, G., Newbery, D., \& Songwe, N. (kein Datum). Rainfall input,thtroughfall and stemflow in a central African rain forest dominatedby ectomycorrhizal trees. Biogeochemestry, 67, S. 73-91.

Cinderby, S., Cambridge, H. M., Herrera, R., Hicks, W. K., Kuylenstierna, J. C., Murray, F., et al. (1998). Global assessment of ecosystem sensitivity to acidic deposition. Stockholm Environment Institute .

Clark, D. A. (2004). Sources or sinks? The responses of tropical forests to current and future climate and atmospheric composition. Phil. Trans. R. Soc. Lond, 359, S. 477-491.

Clough, Y., Abrahamczyk, S., Adams, M., Anshary, A., Arianty, N., Betz, L., et al. (2010). Biodiversity patterns and trophic interactions in human-dominated tropical landscapes in Sulawesi (Indonesia): plants, arthropods and vertebrates. In T. Tscharntke, C. Leuschner, E. Veldkamp, H. Faust, E. Guhardja, \& A. Bidin (Hrsg.), Tropical Rainforest and Agroforests under Global Change. Berlin: Springer-Verlag.

Corre, M. D., Dechert, G., \& Veldkamp, E. (2006). Soil Nitrogen Cycling following Montane Forest Conversion in Central Sulawesi, Indonesia. (70), . Soil Sci.Soc.Am.J , S. 359-366.

Crabtree, R. W., \& Trudgill, S. T. (1981). The use of ion-exchange resin in monitoring the calcium, magnesium, sodium and potassium contents of rainwater. J. Hydrol. (53), S. 361-365.

Crutzen, P. J., Medina, E., Da rocha, G. O., \& Allen, A. G. (2005). Influence of agricultural biomass burning on aerosol size distribution and dry deposition in southeastern of Brazil. Environ. SCi: Technol. , S. 3.

Cuevas, E., \& Medina, E. (1986). Nutrient Dynamics withinAmazonian Forest Ecosystems.1.Nutrient Flux in fine Litter Fall and Efficiency of nutrient utilization. Oecologia (3), S. 466-472.

Curtis, J. J., Clarke, S., Battarbee, G., Kernan, R. W., Catalan, M., \& al., e. (2009). Regional influence of acid deposition and climate change in European mountain lakes assessed using diatom transfer functions. , 54, 2555-2572. Freshw. Biol.

Da rocha, G. O., Allen, A. G., \& Cardoso, A. A. (2005). Influence of agricultural biomass burning on aerosol size distribution and dry deposition in southeastern of Brazil. Environ. SCi: Technol. , 39 (14), 5293-5301.

Dechert, G., Veldkamp, E., \& Brumme, R. (2005). Are partial nutrient balances suitable to evaluate nutrient sustainability of land use systems? Results from a case study in Central Sulawesi, Indonesia. Nutrient Cycling in Agroecosystems (72), S. 201-212.

Dezzo, N., \& Chacon, N. (2006). Nutrient fluxesin incident rainfall,throughfall, and stemflow in adjacent primary and secondary forests of the Gran Sabana, southern Venezuela. Forest Ecology and Management (234), S. 218-226.

Eklund, T. J., \& Pringle, C. M. (1997). Seasonal variation of tropical precipitation chemistry: La Selva, Costa Rica. Atmos. Environ 31 (23), S. 3903-3910.

Erasmi, S., Ardiansyah, M., Propastin, P., \& Huete, A. (2010). Spatiotemporal trends of forest cover change in southeast Asia. In T. L. Tscharntke, E. Veldkamp, H. Faust, E. Guhardja, \& A. Bidin (Hrsg.), Tropical rainforests and Agroforests under Global Change. Heidelberg: Springer.

Erasmi, S., Knieper, C., Twele, A., \& Kappas, M. (2007). Monitoring inter annual land cover dynamics at the rainforest margin in Central Sulawesi, Indonesia. In S. Bochenek (Hrsg.), New Developments and Challenges in Remote Sensing (S. 297-308). Rotterdam: Milpress. 
Erasmi, S., Twele, A., Ardiansyah, M., Malik, A., \& Kappas, M. (2004). Mapping deforestation and land cover conversion at the rainforest margins in Central Sulawesi, Indonesia. EARSeL eProceedings , 3/3, 288-297., S. 288-297.

FAO: Food and Agriculture Organization of the United Nations. (2010). Global Forest Resources Assesment. Rom.

FAO: Food and Agriculture Organization of the United Nations. (2011). The state of World's Forests. Rom.

Fan, J. L., Hu, Z. Y., Wang, T. J., J, Z., Wu, C. Y., \& et al.. (2009). Atmospheric inorganic nitrogen deposition to a typical red soil forestland in south-eastern China. Environ. Monit. Assess. , 159, S. 241-253.

Fenn, M. E., \& Poth, M. A. (2004). Monitoring nitrogen deposition in throughfall using lon Exchange Resin columns: A field test in the San Bernardino Mountains. J. Environ. Qual., (33), S. 2007-2014.

Fenn, M. E., Poth, M. A., \& Arbaugh, M. J. (2002). A throughfall collection method using mixed bed ion exchange columns. The Scientific World Journal , 2, S. 122-130.

Filoso, S., Wiliams, M., \& Melack, J. (1999). Comparison and deposition of throughfall in a flooded forest archipelgo (Negro River Brazil). Biogeochemestry , 45, S. 169-195.

Fischer, E. (2004). Einfluss der Landnutzung auf den Nährstoffhaushalt im Teileinzugsgebietdes Hana an der Grenze des Taï-Nationalparks (Côte d'Ivoire). Ein Vergleich zwischenWald und Kakaoplantagen. dissertation Universität Göttingen.

Fischer, E., Bock, M., \& Gerold, G. (2008). Nutrient in and output fluxes of an agroforested subcatchment of the Hana stream at the border of Taï National Park, Côte d'Ivoire. Hamburger Beiträge zur Physischen Geographie und Landschaftsökologie , 19, S. 49-58.

Formenti, P., Andreae, M. O., Lange, L., Roberts, G., Cafmeyer, J., Rajta, I., et al. (2001). Saharan dust in Brazil and Suriname during large-scale biosphere-atmosphere experiment in Amazonia (LBA)cooperative LBA regional experiment (Claire) in March 1998. Journal of Geophysical Research , 106 (D14), S. 14919-14934.

Gassmann, A. (2004). Extensives Monitoring von Stoffflüssen in Waldökosystemen. Dissertation an der Fakultät für Forst- und Umweltwissenschaften. Albert-Ludwigs-Universität, Freiburg.

Germer, S., Neill, C., Krusche, A., Neto, S., \& and Elsenbeer, H. (2007). Seasonal and withinevent dynamics of rainfall and throughfall chemestry in an open tropical rainforestin Rondonia Brazil. Biogeochemestry, 86, S. 155-174.

Gerold, G. (2009). Different land use impacts in tropical rainforests-Soil degradation and consequences for land rehabilitation. GeoEcology , 40, S. 109-118.

Gerold, G. (2010). Soil and water degradation following forest conversion in the humid tropics (Indonesia). In P. Zdruli, M. Pagliai, S. Kapur, \& A. Faz Cano (Hrsg.), Land Degradation and Desertification : Assessment, Migigation and Remedation (S. 267-283). Berlin: Springer-Verlag .

Giambelluca, T., \& Gerold, G. (2011). Hydrology and biogeochemistry of tropical montane cloud Forests. In D. Levia, D. Carlyle-Moses, \& T. Tanaka (Hrsg.), Forest Hydrology and Biogeochemistry. Ecological Studies 216 (S. 221-259). Springer. 
Giglio, L., Descloitres, J., Justice, C. O., \& Kaufman, Y. J. (2004). An enhanced contextual fire detection algorithm for MODIS. Remode Sensing of Environment, 87, S. 273-282.

Gravenhorst G, K. H.-H. (2000). Trockene und nasse Deposition von Spurenstoffen aus Atmosphäre. In R. Guderian (Hrsg.), Handbuch der Umweldveränderungen und Ökotoxikologie (1b: Atmosphäre Ausg.). Berlin: Springer .

Gunawan, D. (2006). Atmospheric Variability in Sulawesi, Indonesia -Regional Atmospheric Model Results and Observations.

Gutzler, C. (2011). Auswirkungen von Landnutzungsänderungen in den feuchten Tropen auf lokale Wasser- und Nährstoffkreisläufe. Dissertation, Universität Göttingen.

Gutzler, C., Koehler, S., \& Gerold, G. (2010). A comparison of throughfall rate and nutrient fluxes in rainforest and cacao plantation in Central Sulawesi, Indonesia. In T. Tscharntke, C. Leuschner, E. Veldkamp, \& e. a. (Ed.), Tropical Rainforests and Agroforests under Global Change. Heidelberg: Springer.

Hafkenscheid, R. (2002). Hydrology and Biogeochemestry of tropical montane rainforests of contrasting stature in the Blue Mountains,Jamaica. Dissertation,Vrije Universiteitte Amsterdam.

Hammer, O., Harper, D. A., \& Ryan, P. D. (2001). Past:Paleontological Statistic software package for education and data anayisis. Palaeontologia Electronica , 4 (1), 9.

Herrmann, L., Jahn, R., \& Maurer, T. (2010). Mineral dust around the Sahara-from source to sink. A review with emphasis on contribution of the German soil science community in the last twenty years. J.Plant Nutr.Soil Sci. (173), S. 811-821.

Herrmann, R. (1977). Einführung in die Hydrologie. Teubner Studienbücher.

Hetzel, F. (1999). The water and nutrient cycle in a tropical rain forest and a cocoa plantation in Cote d'Ivoire (Bd. 2). Göttingen.

Hofhansel, F., Wanek, W., Drage, S., Huber, W., Weissenhofer, A., \& Richter, A. (2011). Topography strongly affects atmospheric deposition and canopy processes in different types of wert lowland rainforest, Southwest Costa Rica. Biogeochemestry, 106, S. 371-396.

Ibrom, A., Oltchev, A., June, T., Kreilein, H., Rakkibu, G., Ross, T., et al. (2008). Measured PARuseefficiences of photosynthesis in a mountainous tropical rain forest in Indonesia. Tree physiology, 28, S. 499-508.

Jaramillo, V. J., Ahed-Hernández, R., \& Kauffman, J. B. (2003). Root biomass and carbon in a tropical evergreen forest of Mexico: changes with secondary succession and forest conversion to pasture. Journal of Tropical Ecology (19), S. 457-464.

Juhrband, J., Duwe, T., Barkmann, J., Gerold, G., \& Marggraf, R. (2010). Structure and management of cocoa agroforestry systems in Central Sulawesi across an intensification gradient. In T. Tscharntke, C. V. Leuschner, H. Faust, E. Guhardja, \& A. Bidin (Hrsg.), Tropical Rainforest and Agroforests under. Berlin: Springer-Verlag.

Kessler, M., Kessler, P., Gradstein, S., Bach, K., SchmullL, M., \& Pitopang, R. (2005). Tree diversity in primary forest and different land use systems in Central Sulawesi, Indonesia. Biodiversity and Conservation (14), S. 547-560. 
Kleinhans, A. (2003). Einfluß der pedo-hydrologischen Gebietsvarianz auf Wasser- und Nährstoffumsatz in Einzugsgebieten. Dissertaiton Universität göttigen.

Köhler, S., Jungkunst, H. F., Gutzler, C., Herrera, R., \& Gerold, G. (2012a). Atmospheric ionic deposition in tropical sites of Central Sulawesi determined by ion-exchange resin collectors and bulk water collector. water air and soil pollution .

Köhler, S., Jungkunst, H., Erasmi, S., \& Gerold, G. (2012b). Regional Atmospheric Nutrient Depositions in Central Sulawesi. Erdkunde .

Köppen, W. (1923). Die Klimate der Erde. Berlin: Walter der Gruyter Verlag.

Krupa, S. V., \& Legge, A. H. (2000). Passive sampling of ambient, gaseous air pollutants: an assessment from an ecological perspective. Environmental Pollution , 107, S. 31-45.

Krusche, C. A., Cerri, C. E., V, B. M., Lara, L. B., \& Victoria de, R. L. (2003). Acid rain and nitrogen deposition in a sub-tropical watershed (Piracicaba) ecosystem consequences. Environmental Pollution , 121, S. 389-399.

Lacaux, J. P., Delmas, R., Kouadio, G., Cros, B., \& Andreae, M. O. (1992). Precipitation chemistry in the Mayombé Forest of equatorial Africa. J. Geophys. Res (97), S. 6195-6206.

Lanfer, N. (2003). Landschaftsökologische Untersuchungen zur Standortbewertung und Nachhaltigkeit von Agroökosystemen im Tieflandregenwald Ecuadors (Bd. 9). Aachen: EcoRegio,Shaker Verlag.

Larssena, T., \& Carmichaelb, G. R. (2000). Acid rain and acidification in China: the importance of base cation deposition. Environmental Pollution, 110, S. 89-102.

Leemhuis, C. (2005). The Impact of El Niño Southern Oscillation Events on Water Resource Availability in Central Sulawesi, Indonesia. A hydrological modelling approach. Dissertation, Universität Göttingen.

Lilienfein, J., \& Wilcke, W. (2004). Water and element input into native, agri- and silvicultural ecosystems of the Brazilian savanna. Biogeochemistry, 67(2), 183-212.

Luizao, R. C., Luizao, F. J., Pavia, R. Q., Monteiro, F, T., Sousa, L. S., et al. (2004). Variation of carbon and nitrogen cycling processes along a topographic gradient in a central Amazonian forest. Global Change Biology, 10, S. 592-600.

Mackensen, J., Ampt, J., Garrelts, A., Kortekaas, M., \& Veldkamp, E. (1999). Repost on reconnaissance soil survey in the Napu and Sopu-valley, Central Sulawesi,Indonesia. Göttingen: SFB 1687 Datensatz. Maertens, M., Zeller, M., \& Birner, R. (2006). Does technical progress in agriculture have a forest saving or a forest clearing effect? Theory and Evidence from Central Sulawesi. In G. Gerold, M. Fremerey, \& E. Guhardja (Hrsg.), Land use, nature conservation (S. 180-197). Berlin: Springer Verlag. Mahowald, N. M., Artaxo, P., Baker, A. R., Okin, G. S., Gregory, S. R., \& Townsend, A. R. (2005). Impacts of biomass burning emissions and landuse change on Amazonian atmopheric phosphorus cycling and deposition. Global. Biogeochem. Cycles, 19, GB4030.

Mayer, R., Liess, S., Lopes, M. I., \& Kreuzer, K. (2000). Atmospheric Pollution in a tropical rain forest: effects of deposition upon biosphere and hydrosphere II. Fluxes of chemicals and element budgets. Water Air Soil Pollut , 121, 79-92. 
McDowell, W., \& Asbury, C. (1994). Export of Carbon, Nitrogen, and Major Ions from 3 Tropical Montane Watersheds. Limnology and Oceanography (39), S. 111-125.

Muoghalu, J. I. (2003). Contributions of throughfall, stemflow and litterfall to nutrient cycling in a secondary lowland rain forest in lle-Ife, Nigeria. Journal of Tropical Forest Sience , 15 (3), 399-410.

Nadkarni, N., Schaefer, D., Matelson, T., \& al, e. (2004). Biomass and nutrient pools of canopy and terrestrial components in a primary and secondary montane cloud forest, Costa Rica. For. Eco. Manage. , 198, S. 223-236.

Nicklas, U. (2006). Nährstoffeintrag durch Bestandesniederschlag und Pflanzenstreu in KakaoAgroforstsystemen in Zentral Sulawesi, Indonesien, Diplomarbeit, Geographie, Abteilung Landschaftsökologie, Universität Göttingen.

Odum, E. (1999). Ökologie. Grundlagen, Standorte, Anwendung. (O. E. P., Hrsg.) Stuttgart: Thieme Verlag, 3. Auf.

Oziegbe, M., Moughalu, J., \& oke, S. (2011). Litterfall, precipitation and nutrient fluxes in a scondary lowland rain forest in Ile-Ife,Niegeria. Acta Botanica Brassilia , 25, S. 664-671.

Parker, G. (1983). Throughfall and stemflow in the forest nutrient cycle. Advances in Ecological Research (13), S. 57-133.

Phoenix, G. K., Cinderby, S., Kuylenstierna, J. C., Stoc, W. D., Dentener, F. J., \& al, e. (2006). Atmosperic nitrogen deposition in world biodiversitiy hotspots: The need for a greater global perspective in assessing N deposition impacts. Global Change Biology , 12, 470-476.

Proctor, J. (1987). Nutrient cycling in primary and old secondary rainforests. Applied Geography (7), S. $135-152$.

Pye, H. O., Liao, H., Wu, S., Mickley, L. J., Jacob, D. J., Henze, D. J., et al. (2009). Effect of changes in climate and emissions on future sulfate-nitrate-ammonium aerosol levels in the United States. J.Geophys.Res.D. Atmos. (114.), S. D01205.

Rockström, J., Steffen, W., Noone, K. P., Chapin, F. S., Lambin, E. F., \& al., e. (2009). A safe operation space for humanity. Nature (461), S. 472-475.

Rollenbeck, R. (2010). Global Sources-Local impacts:Natural and antropogenic sources of matter deposition in the andes of ecuador. Geoöko, 21, S. 5-27.

Scheer, M. (2011). Mineral nutrient fluxes in rainfall and in a lowland Atlantic rainforest in southern Brazil. Journal of Forest Research, 16, S. 76-81.

Schroth, G., Elias, M., Seixas, R., \& Zech, W. (2001). Nutrient fluxes in rainfall,troughfall and stemflow in tree-based land use systems and spontaneous tree vegetation of central Amazonia. Ecosystems and Environment, 87, S. 37-49.

Schulze, C., Steffan-Dewenter, I., \& Tscharntke, T. (2004). Effects of land use on butterfly communities at the rain forest margin: a case study from Central Sulawesi. In G. Gerold, M. Fremerey, \& E. Guhardja (Hrsg.), Land use, nature conservation and the stability of rainforest margins in Southeast Asia (S. 282-297). Berlin.

Simkin, S. M., Lewis, D. N., Weathers, K. C., Lovett, G. M., \& Schwarz, K. (2004). Determination of sulfate, nitrate, and chloride in throughfall using ion-exchange resins. Water Air Soil Pollut (153), S. 343-354. 
Steffan-Dewenter, I., Kessler, M., Barkmann, J., Bos, M. M., Buchori, D., Erasmi, S.,Faust, H., Gerold, G., Glenk, K., Gradstein, S. R., Guhardja, E., Harteveld, M., Hertel, D., Höhn, P., Kappas, M., Köhler, S., Leuschner, C., Maertens, M., Marggraf, R., Migge-Kleian, S., Mogea, J., Pitopang, R., Schaefer, M., Schwarze, S., Sporn, S. G., Steingrebe, A., Tjitrosoedirdjo, S. S., Tjitrosoemito, S., Twele, A., Weber, R., Woltmann, L., Zeller, M., \& Tscharntke, T. (2007).Tradeoffs between income, biodiversity, and ecosystem functioning during tropical rainforest conservation and agroforestry intensification. PNAS, 104 (12), S. 4973-4978.

Sundarambal, P., Balasubramanian, R., Tkalich, P., \& He, J. (2010). Impact of biomass burning on surface water quality in southeast asia through atmospheric deposition: field observation. Atmospheric Chemistry and Physics Discussions, 10, S. 7745-7778.

Templer, P. H., \& Weathers, K. (2011). Use of mixed ion exchange resin and the denitrifier method to determine isotopic values of nitrate in atmospheric deposition and canopy throughfall. . Atmospheric Environment (45), S. 2017-2020,.

Tukey, H. (1970). The leaching of substances from plants. Annual Review of Plant Physiology (21), S. 305-324.

Veldkamp, E., Purbopuspito, J., Corre, M. D., Brumme, R., \& Murdiyarso, D. (2008). Land use change effects on trace gas fluxes in the forest margins of Central Sulawesi, Indonesia. J. Geophys. Res. G. Biogeosci. (113), S. G02003 (11 pp.).

Veneklaas, E. J. (1990). Nutrient Fluxes in Bulk Precipitation and Throughfall in Two Montane Tropical Rain Forests, Colombia. Journal of Ecology , 78 (4), 974-992.

Villenneuve, M., Gunawan, W., Cornee, J.-J., \& Vidal, O. (2002). Geologie of the central Sulawesi belt (eastern Indonesia): constraints for geodynamic models. International Journal of Earth Sciences , S. 524-537.

Vitousek, P. M., \& Sanford, R. L. (1986). Nutrient cycling in moist tropical forest. Ann. Rev. of Ecol. and Systemat. , 17, S. 137-167.

Waltert, M., Langkau, M., Maertens, M., Härtel, M., Stefan, E., \& Mühlenberg, M. (2004). Predicting the loss of bird species from deforestation in Central Sulawesi. In G. Gerold, M. Fremerey, \& E. Guhardja (Hrsg.), Land use, nature conservation and the stability of rainforest margins in Southeast Asia (S. 327-349). Berlin.

Weber, R. (2005). Kulturlandschaftswandel während des 20. Jh. in Zentralsulawesi - eine historischgeographische Analyse der Lore-Lindu-Bergregenwaldregion. Dissertation. Georg-August-Universität Göttingen.

Whitmore, T. C. (1993). Tropische Regenwälder: eine Einführung. Heidelberg, Berlin, New York.

Whitten, A. J., Mustafa, M., Henderson, G. S., \& (Hrsg). (2002). The ecologie of Sulawesi in The ecologie of Indonesia (Bd. 4).

Williams, M., Fisher, T. R., \& Melack, J. M. (1997). Chemical composition and deposition of rain in the central Amazon, Brazil. Atmos. Environ, 31 (2), 207-217. 


\section{Lebenslauf}

Name:

Geburtsdatum:

Geburtsort:

Staatsangehöhrigkeit:

Schulausbildung:

1991-1994

\section{Studium}

$10 / 1994-03 / 2000$

$04 / 1998-09 / 1998$
Stefan Thomas Köhler

11.10.1967

Worms am Rhein

Deutsch

Aufbaugymnasium Alzey

Allgemeine Hochschulreife; erworben im 2.Bildungsweg

\section{Universität Hohenheim}

Studium der allgemeinen Agrarwissenschaften

Vertiefungsrichtung Pflanzenbau mit Schwerpunkt Bodenkunde

Diplomarbeit: Vergleich verschiedener Messsysteme zur Erfassung von Nährstoffverlusten in Böden Vietnams

Stipendium der Eiselenstiftung; 6 monatiger Studienaufenthalt in Nordvietnam

\section{Berufserfahrung}

$08 / 1984-02 / 1998$

$02 / 1989-07 / 1991$

$04 / 2000-09 / 2002$

$10 / 2002-10 / 2003$

$10 / 2003-12 / 2000$

01/2009 - heute
Ausbildung zum Chemielaboranten bei der Firma BASF

Chemielaborant bei der Firma BASF

Projektleiter im Gutachterbüro TerrAquat Stuttgart

wissenschaftlicher Mitarbeiter am Institut für Bodenkunde der Universität Hohenheim

Laborleiter der zentralen Laboreinheit des SFB 552 der Universität Göttingen in Indonesien

Laborleiter am Lehrstuhl für Landschaftsökologie und Standortkunde an der Universität Rostock

Veröffentlichungen vor der Promotion:

Bischoff, W. -A; Köhler, S.; Kaupenjohann, M. (1996): Variabilität flächenhafter Austräge von Nitrat unter landwirtschaftlicher Nutzung. In: Mitteilgn. Dtsch. Bodenkundl. Gesellsch, 96(1),61-62. 
Gutzler, C.; Koehler, S.;Gerold, G. (2010): A comparison of throughfall rate and nutrient fluxes in rainforest and cacao plantation in Central Sulawesi, Indonesia. In Tscharntke, T.; Leuschner, C.; Veldkamp, E., et al. (Ed.) Heidelberg: Springer.

Köhler, S.; Bischoff, W. -A; Liebig, H. -P (2002): Cultandüngung - ein Beitrag zum Grundwasserschutz durch Verringerung des Nitrataustrages. In Kücke, M. (Ed.) Agricultural Research (2003). Forschungsanstalt für Landwirtschaft, Braunschweig. Landbauforschung Völkenrode FAL, Sonderheft 245, 117-127

Steffan-Dewenter, I., Kessler, M., Barkmann, J., Bos, M. M., Buchori, D., Erasmi, S.,Faust, H., Gerold, G., Glenk, K., Gradstein, S. R., Guhardja, E., Harteveld, M., Hertel, D., Höhn, P., Kappas, M., Köhler, S., Leuschner, C., Maertens, M., Marggraf, R., Migge-Kleian, S., Mogea, J., Pitopang, R., Schaefer, M., Schwarze, S., Sporn, S. G., Steingrebe, A., Tjitrosoedirdjo, S. S., Tjitrosoemito, S., Twele, A., Weber, R., Woltmann, L., Zeller, M., \& Tscharntke, T. (2007).Tradeoffs between income, biodiversity, and ecosystem functioning during tropical rainforest conservation and agroforestry intensification. PNAS , 104 (12), S. 4973-4978. 